\title{
Interference Alignment and Cancellation in Wireless Communication Systems
}

by

Refik Fatih Ustok

\author{
A thesis \\ submitted to the Victoria University of Wellington \\ in fulfilment of the \\ requirements for the degree of \\ Doctor of Philosophy \\ in Engineering.
}

Victoria University of Wellington

2016 



\section{Abstract}

The Shannon capacity of wireless networks has a fundamental importance for network information theory. This area has recently seen remarkable progress on a variety of problems including the capacity of interference networks, X networks, cellular networks, cooperative communication networks and cognitive radio networks. While each communication scenario has its own characteristics, a common reason of these recent developments is the new idea of interference alignment. The idea of interference alignment is to consolidate the interference into smaller dimensions of signal space at each receiver and use the remaining dimensions to transmit the desired signals without any interference. However, perfect alignment of interference requires certain assumptions, such as perfect channel state information at transmitter and receiver, perfect synchronization and feedback. Today's wireless communication systems, on the other hand, do not encounter such ideal conditions. In this thesis, we cover a breadth of topics of interference alignment and cancellation schemes in wireless communication systems such as multihop relay networks, multicell networks as well as cooperation and optimisation in such systems. Our main contributions in this thesis can be summarised as follows

- We derive analytical expressions for an interference alignment scheme in a multihop relay network with imperfect channel state information, and investigate the impact of interference on such systems where interference could accumulate due to the misalignment at each hop.

- We also address the dimensionality problem in larger wireless communication systems such as multi-cellular systems. We propose precoding schemes based on maximising signal power over interference and noise. We show that these precoding vectors would dramatically improve the rates for multi-user cellular networks in both uplink and downlink, without requiring an excessive number of dimensions. Furthermore, we investigate how to improve the receivers which can mitigate interference more efficiently.

- We also propose partial cooperation in an interference alignment and cancellation scheme. This enables us to assess the merits of varying mixture 
of cooperative and non-cooperative users and the gains achievable while reducing the overhead of channel estimation. In addition to this, we analytically derive expressions for the additional interference caused by imperfect channel estimation in such cooperative systems. We also show the impact of imperfect channel estimation on cooperation gains.

- Furthermore, we propose jointly optimisation of interference alignment and cancellation for multi-user multi-cellular networks in both uplink and downlink. We find the optimum set of transceivers which minimise the mean square error at each base station. We demonstrate that optimised transceivers can outperform existing interference alignment and cancellation schemes.

- Finally, we consider power adaptation and user selection schemes. The simulation results indicate that user selection and power adaptation techniques based on estimated rates can improve the overall system performance significantly. 


\section{Acknowledgments}

Undertaking this $\mathrm{PhD}$ has been a truly life-changing experience for me and it would not have been possible to do without the support and guidance that I received from many people.

I would like to first say a very big thank you to my supervisors, Dr. Pawel Dmochowski and Prof. Mansoor Shafi, for all the support and encouragement they gave me during my studies. Without their guidance and constant feedback this $\mathrm{PhD}$ would not have been achievable. Furthermore I would like to thank Prof. Peter Smith for his valuable supervision, even though he was not my supervisor officially. Besides my supervisors, I would like to thank the rest of my thesis committee: Dr. Bryan Ng, Dr. Graeme Woodward and Prof. Jinhang Yuan for their insightful comments and encouragement, but also for the hard questions which incented me to widen my research from various perspectives. I also gratefully acknowledge the funding received towards my $\mathrm{PhD}$ from the Victoria University of Wellington Faculty of Graduate Research.

I would also like to thank my colleagues Jawad Mirza, Callum Neil and Harsh Tataria for all their help, support and friendship. I wish to thank all faculty and staff at Victoria who are providing such a great teaching and research environment for students. Studying at Victoria was an amazing part of my life regarding my both career and personal life, it have given me an instructive and wonderful experience that I will never forget.

I am indebted to many people in Wellington who became my family here. Studying towards a PhD was also an emotional journey that caused ups and downs in my life. Here I first want to thank Brad Lawson, who has helped me make Wellington my home since my first day in Wellington. My life in Wellington would definitely be more difficult without him. Juan Pablo Lampe, my Argentinian brother, was always there whenever I needed help or support. Many thanks for knowing when something is wrong with me, always giving me the extra push I need and simply being a brother from another mother. I also would like to thank Liviu Sas, Snehal Poojary and Peter Godden who all helped me in numerous ways during various stages of my PhD. Very special thanks to Marilena Kouklaki, "H agaph mou kai to mwro mou", for patiently proofreading my thesis before submission and dealing with me during the most stressful times of 
this journey.

I would also like to say a heartfelt thank you to my mum Nurefsan Ustok, my father Dr. Nezih Ustok and my sister Dr. Isik Ustok for always believing in me and encouraging me to follow my dreams. They always give me their love and support in all aspects of my life and I can never afford to thank them enough.

Refik Fatih Ustok

Victoria University of Wellington 


\title{
Notation and Symbols
}

\author{
$\mathcal{N}\left(\mu, \sigma^{2}\right) \quad$ Normal distribution with mean $\mu$ and variance $\sigma^{2}$ \\ $\mathcal{C N}\left(\mu, \sigma^{2}\right) \quad$ Complex normal distribution with mean $\mu$ and variance $\sigma^{2}$ \\ $|\cdot| \quad$ Absolute value \\ $\|\cdot\| \quad$ Euclidean norm of a vector \\ $\|\cdot\|_{F} \quad$ Frobenius norm of a matrix \\ $\mathbb{E}[\cdot] \quad$ Expected value \\ $\operatorname{det}(\cdot) \quad$ Determinant of a matrix \\ $\operatorname{tr}(\cdot) \quad$ Trace of a matrix \\ $(\cdot)^{T} \quad$ Matrix or vector transpose \\ $\operatorname{null}(\cdot) \quad$ Null space of a vector \\ $\operatorname{span}(\cdot) \quad$ The space spanned by the column vectors of a matrix \\ $\operatorname{rank}(\cdot) \quad$ Rank of a matrix \\ $(\cdot)^{-1} \quad$ Inverse of a matrix \\ $(\cdot)^{*} \quad$ Conjugate transpose of a matrix and a vector \\ $\Re \quad$ Real part of a complex number \\ $\Im \quad$ Imaginary part of a complex number
}


$\begin{array}{ll}f(x) & \text { Probability density function(pdf) of } x \\ F(x) & \text { Cumulative distribution function (cdf) of } x\end{array}$

I

Identity matrix

$N_{t} \quad$ Number of antenna at the transmitter

$N_{r} \quad$ Number of antenna at the receiver

$\mathbf{H}_{j k} \quad$ Channel from transmitter $j$ to receiver $k$

$\mathbf{G}_{j k} \quad$ Cross - Channel from transmitter $j$ (out of cell) to receiver $k$

$\hat{\mathbf{H}}_{j k} \quad$ Erroneous channel from transmitter $j$ to receiver $k$

$\hat{\mathbf{G}}_{j k} \quad$ Erroneous cross-channel from transmitter $j$ to receiver $k$

v Precoding vector (Precoder)

$\mathbf{c}_{i} \quad$ The $i$ th codebook.

$\overline{\mathbf{P}} \quad$ Secondary precoding matrix (Secondary precoder)

$\hat{\mathbf{v}} \quad$ Erroneous precoding vector

$\mathbf{u} \quad$ Postcoding vector (Postcoder)

$\hat{\mathbf{u}} \quad$ Erroneous postcoding vector

$P_{t} \quad$ Transmit signal power

$P_{r} \quad$ Received signal power

$d \quad$ Distance between transmitter and receiver

$h_{\mathrm{BS}} \quad$ Height of the base station

$h_{\mathrm{MS}} \quad$ Height of the mobile station 


\begin{tabular}{|c|c|}
\hline$f_{c}$ & Carrier frequency \\
\hline$\sigma_{\mathrm{SF}}$ & Shadowing standard deviation \\
\hline$\gamma$ & The ratio of remaining over dominant interference \\
\hline$\tilde{\gamma}$ & The ratio of remaining over dominant interference for [1] \\
\hline$\sigma^{2}$ & Noise variance \\
\hline $\mathbf{n}$ & Additive white Gaussian noise \\
\hline $\mathbf{n}^{[1]}$ & Additive white Gaussian noise in the first hop of [2] \\
\hline $\mathbf{n}^{[2]}$ & Additive white Gaussian noise in the second hop of [2] \\
\hline $\boldsymbol{\eta}_{i}$ & Refence vector for message $i$ \\
\hline $\boldsymbol{\eta}_{i}^{\mathrm{ICI}}$ & Intercell Interference subspace for receiver $i$ \\
\hline$\varsigma$ & The parameter that controls the CSI imperfection \\
\hline$\xi$ & Antenna correlation coefficient \\
\hline$S$ & Number of streams \\
\hline$K$ & Number of users \\
\hline $\mathbf{L}$ & Antenna correlation matrix \\
\hline $\mathrm{R}_{i}$ & Achievable rate for user $i$ \\
\hline$\tilde{\mathrm{R}}_{\alpha}$ & Ergodic mean sum rate for $\mathrm{BS} \alpha$ \\
\hline$\kappa$ & Colouring parameter \\
\hline$\tilde{\kappa}$ & Optimized colouring parameter \\
\hline
\end{tabular}

$\rho_{\alpha, i} \quad$ Received signal power for user i of cell $\alpha$ considering path loss 
$\tilde{\rho}_{a, i} \quad$ Received signal power for user i of cell $a$ for the system of [1]

$\boldsymbol{\Phi}_{\alpha, k} \quad$ Interference covariance matrix

$\overline{\mathbf{\Phi}}_{\alpha, k} \quad$ Expected interference covariance matrix

$\tilde{\xi} \quad$ Design parameter of orthogonality between channels

$\psi_{\alpha, k} \quad$ Power adaptation coefficient

$M_{\mathrm{ST}} \quad$ Number of users selected

$\Psi_{\alpha, k} \quad$ Total transmit power of a BS

$\Lambda \quad$ Cooperation threshold

$\mathrm{INR}_{\mathrm{dom}} \quad$ Dominant interference to noise ratio

$\mathrm{INR}_{\text {rem }} \quad$ Remaining interference to noise ratio

$R_{\tau} \quad$ Choosen rate threshold

$\operatorname{diag}\{a, b, c\}$ Diagonal matrix with diagonal elements of $\mathrm{a}, \mathrm{b}$ and $\mathrm{c}$ 


\section{Glossary of terms}

\section{Time Division Duplex (TDD)}

TDD uses a single frequency band for both transmitting and receiving. It shares that band by assigning alternating time slots.

\section{Frequency Division Duplex (FDD)}

FDD is a technique where separate frequency bands are used at the transmitter and receiver side, therefore the data signals don't interfere with each other.

\section{Time Division Multiple Access (TDMA)}

TDMA is a channel access method for shared medium networks. It allows many users to share the same frequency channel by dividing the signal into separate time slots.

\section{Frequency Division Multiple Access (FDMA)}

FDMA is a channel access method which gives users an individual allocation of one or several frequency bands, or channels.

\section{Interference Alignment (IA)}

Aligning interference in a smaller subspace (interference subspace) using precoding vectors.

\section{Interference Cancellation}

Cancelling interference which is projected into seperate dimensions from the desired signals using the postcoding vectors, which project the desired signal orthogonal to the interference subspace.

\section{Interference Neutralisation}

Removing interference which is aligned into appropriate dimensions subsequently. As discussed in Chapter 3, the interfering signals are neutralized by projecting them in contrasting dimensions to eliminate themselves. 


\section{Channel State Information (CSI)}

In wireless communications, CSI refers to known channel properties of a communication link. This information describes how a signal propagates from the transmitter to the receiver and represents the combined effect of, for example, scattering, fading, and power decay with distance.

\section{Vector Quantization VQ}

$\mathrm{VQ}$ is a classical quantization technique from signal processing that allows the modelling of probability density functions by the distribution of prototype vectors.

\section{Precoding Vector}

Precoding vector is a vector with which the transmitted signal is processed before transmission to the receiver.

\section{Postcoding Vector}

Postcoding vector is a vector with which the received signal is processed in the receiver after transmission.

\section{Intracell Interference}

Interference that is caused by the users of the same cell.

\section{Intercell Interference}

Interference that is caused by out-of-cell users. It is also subcategorized as two: Remaining and dominant interference

\section{Dominant Interference}

Type of intercell interference of which power is assumed to be significantly stronger than other interference. In this thesis, the dominant interference is cancelled with postcoding vectors.

\section{Remaining Interference}

Type of intercell interference of which power is assumed to be lower than other interference. In this thesis, the remaining interference is treated as noise.

\section{Transmit SNR}

Signal-to Noise-Ratio of the transmitted symbol. It is calculated as the energy per bit to noise power at the receiver. However, in some parts of this thesis, the variance of noise is normalized to be 1 . In this case, transmit SNR also refers to transmit power. This concept is also adopted in [3] and in a 
number of papers.

\section{Mean Square Error (MSE)}

It measures the average of the squares of the errors or deviations - that is, the difference between the estimator and what is estimated. 


\section{Contents}

Abstract

$\begin{array}{ll}\text { Acknowledgments } & \text { iii }\end{array}$

Notation and Symbols $\quad$ v

Glossary of terms ix

1 Introduction 1

1.1 Introduction . . . . . . . . . . . . . . . . . 1

1.2 Research Framework . . . . . . . . . . . . . . . . . . 2

1.2.1 Motivation . . . . . . . . . . . . . . 2

1.2 .2 Contributions . . . . . . . . . . . . . . . . . . 4

1.2.3 List of Publications . . . . . . . . . . . . . 6

1.3 Thesis Outline . . . . . . . . . . . . . . . 7

2 Theoretical Background and Preliminary Works 9

2.1 Wireless Channels . . . . . . . . . . . . . . . . . . . 9 9

2.2 Multiple Antenna Systems . . . . . . . . . . . . . . . . . . . . 11

2.2.1 Single User Systems . . . . . . . . . . . . . . . . . . . . . . . . . . . . . . . . 11

2.2.2 Multiuser Systems . . . . . . . . . . . . . . . . 11

2.3 Cellular Networks . . . . . . . . . . . . . . . . . . . . . . . . . 12

2.3.1 Scenarios Considered for the Uplink . . . . . . . . . . . . 14

2.3.2 Scenarios Considered for the Downlink . . . . . . . . 16

2.4 Interference Management Systems . . . . . . . . . . . . . . . 19

2.4.1 Traditional Interference Management Systems . . . . . . 19

2.5 Interference Alignment - The Concept . . . . . . . . . . . . . . . 20

2.6 Interference Alignment in MIMO X Channels . . . . . . . . . . 23

2.6.1 System Model . . . . . . . . . . . . . . . . . 23

2.7 Intereference Alignment in Interference Channels . . . . . . . . 25

2.7.1 System Model . . . . . . . . . . . . . . . . . . 26

2.8 Interference Alignment Challenges . . . . . . . . . . . . . . . . . 28

2.8.1 Dimensionality . . . . . . . . . . . . . . 28 
2.8.2 Channel Estimation and Feedback . . . . . . . . . . . 28

2.8.3 Interference Alignment in Large Scale Networks . . . . . 30

2.8.4 Synchronization . . . . . . . . . . . . . . 30

2.9 Imperfect Channel State Information and Limited Feedback . . 30

2.9.1 Modelling erroneous CSI . . . . . . . . . . . . . . 30

2.9.2 Limited Feedback . . . . . . . . . . . . . . . . . . 31

2.10 Antenna Correlation . . . . . . . . . . . . . . . . . . 32

2.11 Literature Review . . . . . . . . . . . . . . . . . . . . 32

2.11.1 IA Applications . . . . . . . . . . . . . . . . . . 32

2.11.2 Cellular Networks in Uplink . . . . . . . . . . . . . 33

2.11.3 Cellular Networks in Downlink . . . . . . . . . . . . . 34

2.12 Summary . . . . . . . . . . . . . . . . 35

3 Interference Alignment in Multihop Relay Networks $\quad 37$

3.1 Interference Alignment in Multihop Interference Channels . . . . 37

3.1.1 System Model . . . . . . . . . . . . . . . 38

3.1.2 Performance Analysis . . . . . . . . . . . . . . . . . . . . 42

3.1.3 Using Multiple Antennas instead of Symbol Extension . 49

3.1.4 Simulation Results . . . . . . . . . . . . . . . . . 49

3.2 Summary . . . . . . . . . . . . . . . . . . 54

4 Uplink Interference Alignment and Cancellation in Multicell Networks $\quad 55$

4.1 Interference Alignment and Cancellation for Uplink 2-Cell Networks 55

4.1.1 Interference Alignment with Limited Feedback . . . . . . 58

4.1 .2 Simulation Results . . . . . . . . . . . . . . . 60

4.2 Interference Alignment for Multicell Networks in the Uplink . . 61

4.2.1 Interference Alignment with Combined Receivers For Heterogeneous Cellular Networks . . . . . . . . . . . 61

4.2.2 Scenarios Considered for Heterogeneous Networks . . . . 64

4.2 .3 Combined Receivers _. . . . . . . . . . . . . 65

4.2.4 Proposed IA Algorithm of the Combined Receiver . . . . 67

4.2.5 Performance Analysis for Heterogeneous Networks . . . . 69

4.2 .6 Simulation Results . . . . . . . . . . . . . . . 69

4.3 Summary ....................... . . . 73

5 Downlink Interference Alignment and Cancellation in Multicell Networks $\quad 75$

5.1 Interference Alignment for 2-Cell Networks in the Downlink . . 76 5.1 .1 Performance Metrics . . . . . . . . . . . . . . . 79

5.2 Interference Alignment for Multicell Networks in the Downlink . 80 5.2 .1 Design of the Receivers . . . . . . . . . . . . . . . . 83 
5.2.2 Selecting The Colouring Parameter $\kappa \ldots . . . . . .85$

5.2.3 Generating Precoding Vectors . . . . . . . . . . . . . 88

5.2.4 Considering Multiple Dominant Interferers . . . . . . . . 90

5.2 .5 Performance Metrics . . . . . . . . . . . . . . . . . . 91

5.2 .6 Simulation Results . . . . . . . . . . . . . . . . . . . 92

5.3 Cooperation in Interference Alignment and Cancellation . . . . 97

5.3.1 Receiver Architecture . . . . . . . . . . . . . . . . . 99

5.3 .2 Transmitter Architecture . . . . . . . . . . . . . . 100

5.3.3 The Algorithm . . . . . . . . . . . . . . . . . . . . 100

5.3.4 Performance Metrics . . . . . . . . . . . . . . . 102

5.3.5 Simulation Results . . . . . . . . . . . . . . . . 105

5.4 Summary . . . . . . . . . . . . . . . . . . 111

6 Jointly Optimisation of Interference Alignment and Cancellation 113

6.1 Jointly Optimisation of the Transmitters and the Receivers . . 114

6.2 Optimised Transceivers for Multicell Uplink Networks . . . . . . 115

6.2.1 The Algorithms of Optimised Transceivers For Multicell Uplink Networks . . . . . . . . . . . . . . . 116

6.2.2 Summary of the Equations For the Uplink . . . . . . . . 124

6.2.3 Performance Parameters for Uplink Optimised Transceivers 124

6.2.4 Simulation Results . . . . . . . . . . . . . . . . 125

6.3 Optimised Transceivers for Multicell Downlink Networks . . . . 128

6.3 .1 The System Model . . . . . . . . . . . . . . . . . . . . . 129

6.3.2 The Algorithm of the Optimised Transceivers for Downlink Networks . . . . . . . . . . . . . . 130

6.3.3 Summary of the Equations For the Downlink . . . . . . . 132

6.3.4 Simulation Results . . . . . . . . . . . . . . . . 133

6.4 Summary . . . . . . . . . . . . . . . . . 137

7 User Selection and Power Adaptation Techniques 141

7.1 Motivation and Related Work . . . . . . . . . . . . . . . . . . 141

7.2 User Selection Methods . . . . . . . . . . . . . . . . . . . . . . 143

7.2.1 Fair User Selection Algorithms . . . . . . . . . . . . . . . 143

7.2.2 Norm Based User Selection . . . . . . . . . . . . . . . . 144

7.2.3 Semi-Orthogonal User Selection . . . . . . . . . . . . . . 144

7.2.4 SLNR-Based User Selection . . . . . . . . . . . . . . . 144

7.2.5 Estimated Rate User Selection . . . . . . . . . . . . . . 145

7.2.6 Minimum Remaining Interference User Selection . . . . . 145

7.2.7 SLRINR-Based User Selection . . . . . . . . . . . . 145

7.2.8 Simulation Results . . . . . . . . . . . . . . . . 146

7.3 Power Adaptation Methods . . . . . . . . . . . . . . . . . . 147 
7.3.1 Uniform Power Adaptation . . . . . . . . . . . . . . . . . 148

7.3.2 Frobenius Norm Uniform Power Adaptation . . . . . . . 148

7.3.3 Uniform Power Adaptation based on Estimated Rate . . 148

7.3.4 Uniform Power Adaptation based on SLRINR . . . . . . 149

7.3.5 Simulation Results . . . . . . . . . . . . . . . 149

7.4 Summary . . . . . . . . . . . . . . . . . 152

8 Conclusions and Future Work $\quad 155$

8.1 Conclusions . . . . . . . . . . . . . . . . . 155

8.2 Future Work . . . . . . . . . . . . . . . . . . . 159

8.2.1 Analytical Analysis of System Performance Metrics with Imperfect CSI and CCSI . . . . . . . . . . . . . 159

8.2.2 IAC in Next Generation Wireless Communications . . . 160

$\begin{array}{lr}\text { Appendices } & 163\end{array}$

$\begin{array}{ll}\text { A Detailed explanation of (3.20) } & 165\end{array}$

$\begin{array}{ll}\text { B Detailed Explanation of (6.7) } & 167\end{array}$ 


\section{List of Figures}

2.1 Effects of path loss, shadowing and multipath on $P_{r} / P_{t}(\mathrm{~dB})$ ratio with $\log$ distance [18]. . . . . . . . . . . . . . . . . . . . . 10

2.2 Antenna pattern for 3 -sector cells . . . . . . . . . . . . . . 13

2.3 The setting used for cellular network simulations with 19 cells and 3 user in each cell. The snapshot was taken over 1 time-frame. 14

2.4 Average INR for each interfering user in the Uplink . . . . . . . 14

2.5 Received SINR for urban random user locations in uplink cellular networks . . . . . . . . . . . . . . . . . . 15

2.6 Received SINR for urban cell-edge user locations in uplink cellular networks . . . . . . . . . . . . . . . . . 16

2.7 Average INR for each interfering user in the Downlink . . . . . . 17

2.8 Received SINR for urban random user locations in downlink cellular networks . . . . . . . . . . . . . . . . . . . . . . 17

2.9 Received SINR for urban cell-edge user locations in downlink celluar systems . . . . . . . . . . . . . . . . . . . 18

2.10 Received SINR for suburban users in downlink cellular networks 19

2.11 Interference alignment - Toy example of [9] . . . . . . . . . . 21

2.12 Interference alignment with precoding vectors [15] . . . . . . . . 23

2.13 MIMO X channel model of [14]. . . . . . . . . . . . . 25

2.14 Interference alignment in an interference channel of [11] . . . . 26

3.12 user - 2 transmitter - 2 relay - 2 receiver $(2 \times 2 \times 2)$ Network

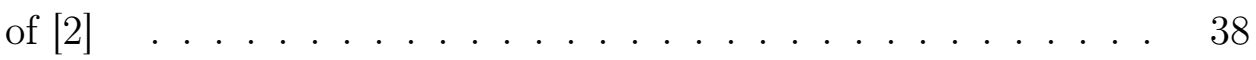

3.2 First hop of the network of $[2] \ldots \ldots \ldots$

3.3 Second hop of the network of [2] . . . . . . . . . . . . . 41

3.4 Misalignment due to the CSI errors in the first hop of [2] . . . . 44

3.5 Misalignment due to the CSI errors in the second hop of [2] . . . 47

3.6 CDF of SNR with perfect CSI and SINR with imperfect CSI for $x_{1,1}$ at relay $1 \ldots \ldots \ldots \ldots \ldots \ldots$

3.7 CDF of interference at relay 1 for $x_{1,1}$ via simulations, analytical analysis and $\log$ normal fitting . . . . . . . . . 50 
3.8 CDF of end-to-end Interference for $x_{1,1}$ symbol via simulations and log-normal fitting. . . . . . . . . . . . . . . 52

3.9 Bit error rates of each symbol with perfect and imperfect CSI cases. 52

3.10 The symbol error rates for receiver 1 with channel estimation error: erroneous precoders at transmitters only, at relays only, at both transmitters and relays. . . . . . . . . . . . .

3.11 IA using symbol extension vs. MIMO, with perfect and imperfect CSI . . . . . . . . . . . . . . . . 53

4.1 System model for 2 user-2 cell uplink MIMO system . . . . . . . 56

4.2 Ergodic mean sum rates for 2 - cell uplink network with imperfect CSI and limited feedback . . . . . . . . . . . . . . . .

4.3 Ergodic mean sum rates for 2 - cell uplink network with imperfect CSI and limited feedback (3 bits only) . . . . . . . . . . . . 61

4.4 System model of the uplink heterogeneous cellular network of [1] 62

4.5 Ergodic mean sum rates for scenario 1 of uplink heterogeneous cellular networks, $N_{t}=3, N_{r}=6$. . . . . . . . . . 70

4.6 Ergodic mean sum rates of both scenario 2 and scenario 3 of uplink heterogeneous cellular networks for $N_{t}=3, \tilde{\gamma}=0.2 \ldots$.

4.7 Ergodic mean sum rates of scenario 2 using combined receiver, Only ZF part of this combined receiver and MMSE receiver $N_{t}=$ $3, \tilde{\gamma}=0.2 \ldots \ldots \ldots \ldots \ldots \ldots \ldots$

4.8 Difference between the sum rates of proposed combined receivers and MMSE receivers for various $\tilde{\gamma}$ ratios, $\mathrm{SNR}=5 \mathrm{~dB}, N_{t}=3$

4.9 CDF of SINR, scenario 2 for correlated and uncorrelated channels, $\tilde{\gamma}=0.2, \quad N_{t}=3 \ldots \ldots \ldots \ldots \ldots$

5.1 System model for 2-cell downlink network of [111] . . . . . . .

5.2 Ergodic mean sum rates for 2-cell downlink network with imperfect CSI . . . . . . . . . . . . . . . . . . 80

5.3 System model of downlink IA in multicellular networks of [3] . . 81

5.4 Simulations for $\gamma=0.4$ showing the variation in optimal $\kappa$ values. $\kappa$ computed from $(5.25)$ is also shown . . . . . . . . 86

5.5 Simulations for $\gamma=0.01$ showing the variation in optimal $\kappa$ values. $\kappa$ computed from $(5.25)$ is also shown . . . . . . . . 86

5.6 Comparison of the precoders with benchmark results of [12]. $\kappa$ is found from $(5.25) \ldots \ldots \ldots . \ldots . \ldots 93$

5.7 Comparison of optimised, variable and fixed $\kappa \ldots . . . . . .993$

5.8 Ergodic mean sum rates with random locations (Scenario 1 Downlink multicell systems ) . . . . . . . . . . .

5.9 Ergodic mean sum rates for random locations with imperfect CSI and CCSI (Scenario 1 - Downlink multicell systems) . . . . . 96 
5.10 Ergodic mean sum rates for cell-edge locations (Scenario 2 Downlink multicell systems) . . . . . . . . . . . .

5.11 Ergodic mean sum rates for cell edge locations with Imperfect CSI and CCSI (Scenario 2 - Downlink multicell systems) . . . . 98

5.12 System model for cooperation in a downlink cellular network . . 99

5.13 Ergodic mean sum rates for urban users with/without cooperation106

5.14 Ergodic mean sum rates for urban users with partial cooperation and imperfect CCSI . . . . . . . . . . . . . . . 106

5.15 The cooperation gain for urban users . . . . . . . . . . 107

5.16 Ergodic mean sum rates for suburban users with/without cooperation . . . . . . . . . . . . . . . . . . . . . 108

5.17 Ergodic mean sum rates for suburban users with partial cooperation and imperfect CCSI . . . . . . . . . . . . . . . 109

5.18 The cooperation gain for suburban users . . . . . . . . . . 109

5.19 The CDF of additional interference that is caused by imperfect CCSI . . . . . . . . . . . . . . . . . . . . 111

6.1 System model for multicell uplink networks . . . . . . . . . . . . 115

6.2 System model of the proposed optimised transceivers for uplink multicell networks . . . . . . . . . . . . . . . . 116

6.3 Ergodic mean sum rates for random user locations (Scenario 1 Uplink multicell networks) . . . . . . . . . . . . . . .

6.4 Ergodic mean sum rates for random user locations with imperfect CSI and CCSI (Scenario 1 - Uplink multicell networks) . . . . .

6.5 Ergodic mean sum rates for cell-edge locations (Scenario 2 - Uplink multicell networks) . . . . . . . . . . . . . .

6.6 Ergodic mean sum rates for cell-edge users with imperfect CSI and CCSI (Scenario 2 - Uplink multicell networks) . . . . . .

6.7 System model of the proposed optimised transceivers for multicell downlink networks . . . . . . . . . . . . . . . . . . . 129

6.8 Comparison of proposed optimised transceivers with benchmark results of $[12] \ldots \ldots \ldots \ldots \ldots$

6.9 Ergodic mean sum rates for random user locations (Scenario 1 Downlink multicell networks) . . . . . . . . . . . . . 135

6.10 Ergodic mean sum rates for cell-edge locations (Scenario 2 Downlink multicell networks) . . . . . . . . . . . . . 136

6.11 Ergodic mean sum rates with imperfect CSI and CCSI for random user locations (Scenario 1 - Downlink multicell networks) . . . .

6.12 Ergodic mean sum rates with imperfect CSI and CCSI for celledge locations (Scenario 2 - Downlink multicell networks) . . . 
7.1 Ergodic mean sum rates of optimized transceivers with user selection methods for random user locations with perfect CSI and CCSI (Scenario 1 - Downlink multicell systems) . . . . . . . . . 146

7.2 Ergodic mean sum rates of optimized transceivers with user selection methods for random user locations with imperfect CSI and CCSI (Scenario 1 - Downlink multicell systems) . . . . . . . . . 147

7.3 Ergodic mean sum rates of optimized transceivers with power adaptation for random user locations with perfect CSI \& CCSI (Scenario 1 - Downlink multicell systems) . . . . . . . . . . . . . 149

7.4 Ergodic mean sum rates of optimized transceivers with power adaptation for random user locations with imperfect CSI \& CCSI (Scenario 1 - Downlink multicell systems) . . . . . . . . . . . 150

7.5 Ergodic mean sum rates of optimized transceivers with power adaptation for cell-edge user locations with perfect CSI \& CCSI (Scenario 2 - Downlink multicell systems) . . . . . . . . . . . . . 151

7.6 Ergodic mean sum rates of optimized transceivers with power adaptation for cell-edge user locations with imperfect CSI \& CCSI151

7.7 Improving the mean sum rates with user selection and power adaptation (Scenario 1 - Downlink multicell systems) . . . . . . 152 


\section{List of Tables}

2.1 Environment parameters for simulations . . . . . . . . . . . . . 12

5.1 Summary of the system for a cooperative downlink cellular network . . . . . . . . . . . . . . . . . . 102

5.2 Outage probability for urban users with/without cooperation . . 107

5.3 Outage probability for suburban users with/without cooperation 110

6.1 Summary of the Equations for Uplink Optimised Transceivers . 124

6.2 Summary of the Equations for Downlink Optimised Transceivers 132 


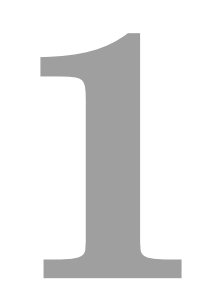

\section{Introduction}

In this chapter, a brief introduction and the research motivation of this dissertation are given. Furthermore, we list our major contributions. The outline of the thesis is provided in the last section of this chapter.

\subsection{Introduction}

Current research in wireless communication technologies is motivated by the ever - increasing demand for high data-rate transmission. As a result of this, the performance of wireless networks has significantly improved in recent years. However, due to the extensive use of smart phones, tablets and multimedia devices, there remains an immediate need for more efficient wireless communications. One of the key issues for wireless communication technologies is the heavily-congested radio spectrum. Due to the limited radio spectrum, many wireless users share the same resources in time and/or frequency. This arrangement results in interference. Communication in the presence of interference has been considered for many networks such as cellular networks, wireless local area networks and wireless ad-hoc networks. In these networks, it is well known that the performance of each user is interference limited [4-8].

There is an extensive literature on interference management techniques. Traditional methods to mitigate the impact of interference often revolve around giving each user exclusive access to a fraction of the communication resources. The main idea behind these methods is limiting the number of overlapping transmissions. However, these methods result in inefficient usage of radio resources. Interference alignment (IA) is a recent concept where the undesired signal component (interference) is aligned to a smaller subspace, called the interference 
subspace [9]. In a network consisting of $K$ users (transmitter-receiver pairs), the principles of IA can enable the users to communicate interference-free for half of the time [9]. Before the development of IA, traditional techniques such as TDMA would allow each user to communicate interference free for $1 / K$ of the time [9]. One drawback of IA is that it relies on certain assumptions. For example, IA requires perfect channel state information (CSI) to align the interference perfectly within the same subspace. However, this is not realistic for practical communication systems which encounter many challenges, such as a limited number of dimensions to align the interference for large scale networks, imperfect synchronization and limited feedback. Hence, it is crucial to take these challenges into account and analyse systems with imperfect conditions.

\subsection{Research Framework}

This section discusses the motivation and the main contributions of the thesis.

\subsubsection{Motivation}

Future mobile wireless networks are faced with an increasing demand for higher data rates. The mobile data volume is predicted to increase 18 times between 2012 and 2017 [10]. Due to the high costs of frequency spectrum these systems need to be extremely efficient in terms of the spectrum usage. Therefore, the focus is on efficient wireless networks where many users share the same radio resources. As a result of the existence of multiple users in the same radio resources, interference is one of the biggest challenges that limits the performance of wireless communications. IA is an inspiring technique, recently proposed in [11] and [12]. In order to understand what IA could achieve, consider the toy example of [13] which is motivated by the wireless interference channel. Let us assume a conference in a room with $K$ speakers, who will present their talks. Each listener must be able to hear his/her desired speaker with no interference from other $K-1$ speakers. In order to be fair to each speaker, we should allow each user equal amounts of time to talk to his/her audience. Considering all of these issues, we should answer the question: What is the maximum amount of time that each speaker can talk while causing no interference to another speaker's audience? Using traditional interference management techniques, one could let each speaker talk for $1 / K$ of the total session time. This corresponds to the idea of time division multiple access (TDMA) in wireless networks. However, it is suggested in [13] that each speaker can speak $1 / 2$ of the total time without causing any interference. This seemingly impossible result can be made possible by the concept of IA which can be achieved in time, frequency or space. However, IA has many practical issues. Here we summarise the problems that 
we have investigated in this thesis.

- It has been shown in the pioneering papers of IA $[9,11,12,14]$ that perfect alignment of interference requires perfect channel estimation and feedback. However, today's communication systems do not encounter such ideal conditions. Therefore it would be beneficial to show analytically that the performance of IA deteriorates with imperfect CSI. The system proposed in [2] is a multi-hop relay network and aligns the interference over two hops. However, perfect CSI is assumed at transmitters, relays and receivers. Any CSI imperfections would cause misalignment and accumulation of interference at the receiver end. Therefore, we initially started our research analytically analysing the system of [2] with perfect and imperfect CSI at each hop of the system.

- Dimensionality is also a very important problem in IA systems [15]. As the interference is aligned using precoding vectors, the system should have enough dimensions to align all interfering sources within a same subspace. This dimensional challenge is relatively mild in the case of multiple-inputmultiple-output (MIMO) systems where more users can be supported as long as the number of antennas grow linearly with network size [15]. However, the number of antennas that can be employed, especially in handsets, is limited. Therefore, it is required to use the available dimensions as efficiently as possible. Motivated by this, the second stage of our research was shifted towards cellular systems where there are many interfering users.

- It is not possible to align all interference terms in bigger networks with only precoding vectors, as a result of the antenna requirements. The authors of [3] proposed to use interference alignment and cancellation (IAC) systems, where they align the intracell interference (interference within the same cell) with precoding vectors and cancel the intercell interference (interference from out of cell) with postcoding vectors. This system is more practical than IA systems as it does not require as many available dimensions as IA systems. Therefore, we have investigated improvements to IAC systems using more efficient preocoding and postcoding vectors.

- Another challenge is the reciprocity and feedback required to generate the precoding vectors because the iteration between the transmitter and the receiver as suggested in [3] would increase the overhead dramatically. Therefore it is very important to find an optimal solution which would reduce the overhead due to the iteration. Using the system considered in [3], we optimised the overall system using a system metric that reduces the overhead significantly. 


\subsubsection{Contributions}

In order to increase the efficiency of spectrum usage, cancelling interference is essential. In this thesis, different aspects of IA and IAC schemes are examined. We begin by investigating the impact of interference on a multihop relay network where interference could accumulate due to the misalignment at transmitters and relays. We analyse the performance of such systems with imperfect CSI and derive the expressions for signal-to noise ratio (SNR) and signal-to-interferencenoise-ratios (SINR).

After studying multihop relay networks, the focus of this research shifts towards cellular systems, which suffer from strong interference. We develop various precoding schemes which improve the rates dramatically for both uplink and downlink systems. Furthermore, we investigate how to improve the receivers to enable them to deal with interference more efficiently.

We also consider optimisation of the system for both uplink and downlink cellular systems. We find the optimum set of transceivers and receivers. Finally we consider power adaptation and user selection schemes which improve system performance significantly.

Our major contributions are listed in three main categories.

\section{IA in Multihop Networks}

Our contributions in multihop networks are as follows.

- We derive analytical expressions for the SNR and SINR at the relays. For the former we show that, due to the zero forcing (ZF) at the relays, the expected value of the noise tends to infinity. Similarly the interference caused by misalignment due to CSI errors is shown to be very prominent with the mean tending to infinity.

- We demonstrate that the SINR analysis for the relays can be extended to the destinations and show that there are cumulative effects of interference and noise at both stages.

- We validate the analysis through system simulations. We show that the interference due to CSI errors can be modelled via simulation by a log normal distribution, thus confirming the presence of prominent instantaneous values of interference.

\section{IAC in Multicell Networks}

We first consider a multicell network in the uplink. We use novel combined receivers which completely null out dominant interference terms while reducing 
the effect of remaining interference and noise. Then we discuss multicell downlink networks and investigate how to design more efficient precoding vectors. We propose novel precoding schemes that would improve system performance dramatically. Later, we study cooperation among adjacent cells with imperfect cross channel state information (CCSI). We derive analytical expressions for additional interference due to misalignment as a result of imperfect CCSI. We summarise some of our contributions for multicell networks as follows.

- We consider an IA scheme for a multicell network in the uplink and propose a novel combined receiver which nulls the dominant interferers with ZF and subsequently uses minimum-mean-square-error (MMSE) processing to reduce the effects of the remaining interference and noise. We show the range of remaining to dominant interference ratios where our receiver outperforms the traditional MMSE receiver.

- We propose novel precoding vectors which reduce the impact of unknown remaining interference and also maximise signal - to - leakage - remaining interference - noise - ratio (SLRINR) for each user.

- We optimise the system colouring parameter (to be defined later) in order to reduce the effects of interference and noise. We investigate how to improve the sum rates using colouring parameters based on user location within the cell where each user is exposed to different interference levels.

- We propose a partial cooperative interference cancellation scheme based on the received powers from adjacent base stations (BS). This enables us to assess the merits of varying mixtures of cooperative and non-cooperative users and the gains achievable while reducing the overhead of CCSI estimation between the interfering BS and the user.

- In order to show the impact of environment type on the cooperation gains, we analyse our system for urban and suburban multicellular environments using the COST 231 Hata model [16]. We demonstrate that the cooperation gains are different for different environments.

- We analytically derive expressions for the additional interference caused by imperfect CCSI and demonstrate its impact on the receiver performance in terms of mean sum rates and cooperation gains.

\section{Optimisation of IAC}

We propose jointly optimisation of IAC for multicell networks in both uplink and downlink and propose optimum transmitter-receiver set to align and cancel the interference. We also apply user selection and power adaptation techniques 
to improve the sum rates. We summarise our contributions on optimisation of IAC techniques as follows.

- For the uplink, we propose two types of optimised transceivers minimizing mean square error (MSE) for the desired cell. First, we consider optimizing the MMSE receiver of [3]. Later, we investigate optimizing with the combined receiver of [1].

- For the downlink, we propose optimised transceivers using an MMSE receiver. However, we do not consider optimisation with combined receivers, as it has been shown in Chapter 4 that the combined receivers are not suitable for downlink systems due to the antenna requirements at the receiver end. We also apply user selection and power adaptation techniques to our optimised IAC system and improve the mean sum rates significantly.

\subsubsection{List of Publications}

The content of this thesis has been partially published, accepted or submitted for publication in the papers listed below.

\section{International Conference Proceedings}

- R. F. Ustok, P. A. Dmochowski, P. J. Smith, M. Shafi, "Aligned Interference Neutralisation for $2 \times 2 \times 2$ Interference Channel with Imperfect Channel State Information", in Proc. of IEEE International Conference on Communications (ICC), pp. 5230-5235, 2013.

- R. F. Ustok, P. A. Dmochowski, P. J. Smith, M. Shafi, "Interference Alignment with Combined Receivers for Heterogeneous Networks", in Proc. of IEEE International Conference on Communications (ICC), pp. 5287$5292,2014$.

\section{International Journal Issues}

- R. F. Ustok, P. A. Dmochowski, P. J. Smith, M. Shafi, "Cooperative Interference Cancellation for Cellular Networks with Imperfect CCSI", Accepted to appear in IET Communications, ISSN:1751-8628, doi: 10.1049/iet.com.2015.0897, 2015.

- R. F. Ustok, P. A. Dmochowski, P. J. Smith, M. Shafi, "Interference Alignment for Cellular Networks with SLRINR precoders", Submitted to IET Communications, 2016. 


\section{Under Preparation}

- R. F. Ustok, P. A. Dmochowski, P. J. Smith, M. Shafi, "Interference Alignment and Cancellation with Optimised Transceivers for Cellular Networks", (Under preparation)

\subsection{Thesis Outline}

The rest of this dissertation is organised as follows. Chapter 2 presents background on the IA concept and the wireless channel model that will be used throughout this thesis. A literature review of IA is also presented in this chapter, giving some IA application examples. Chapter 3 describes IA in multihop relay networks, where we analytically analyse the impact of imperfect CSI on system performance. Chapter 4 includes all the research on the uplink IAC in multicellular networks. Here, we propose a novel postcoding scheme. Chapter 5 discusses the downlink IAC schemes for multicellular systems. Various precoder, postcoding schemes and also cooperation has been proposed in this chapter. Chapter 6 presents optimisation of transmitter-receiver sets for aligning and cancelling interference in both uplink and downlink systems. In Chapter 7 , we discuss improving the system performance with user selection and power adaptation techniques. Finally Chapter 8 concludes this research and provides a discussion about possible future work. 


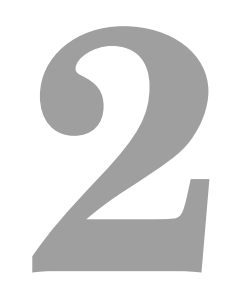

\section{Theoretical Background and Prelim- inary Works}

An overview of wireless systems and preliminary works for IA are presented in this chapter. First, some key concepts of wireless channels are discussed, followed by a detailed explanation of MIMO and cellular networks used in this thesis. We show via simulations that cellular networks suffer from strong interference. This builds a strong motivation for IA schemes. We also discuss the concept of IA and its challenges. We give a literature review of key papers to discuss what has already been done and open problems that we will cover in this thesis.

\subsection{Wireless Channels}

The characteristics of a wireless communication channel between the transmitter and the receiver control the performance of the system. Therefore, it is very important to understand the characteristics of wireless communication channels. Most of the general concepts discussed in this chapter are valid for all kinds of systems studied in this thesis.

A critically important characteristic of the wireless channel is the variations of the channel strength over time and frequency [17]. These variations can be sub-categorised into two.

- Large scale fading, is the variations due to path loss and shadowing that occur over relatively large distances and is typically frequency independent [18]. 
- Small scale fading, is the variations due to multipath, where a signal follows two or more paths to the receiver. This type of fading is experienced over very short distances, on the order of the signal wavelength and is frequency dependent [18].

The effects of large-scale and small-scale fading are illustrated in Fig. 2.1, where $P_{t}$ is the transmit signal power, $P_{r}$ is the receive signal power and the distance between the transmitter and receiver is denoted by $d$.

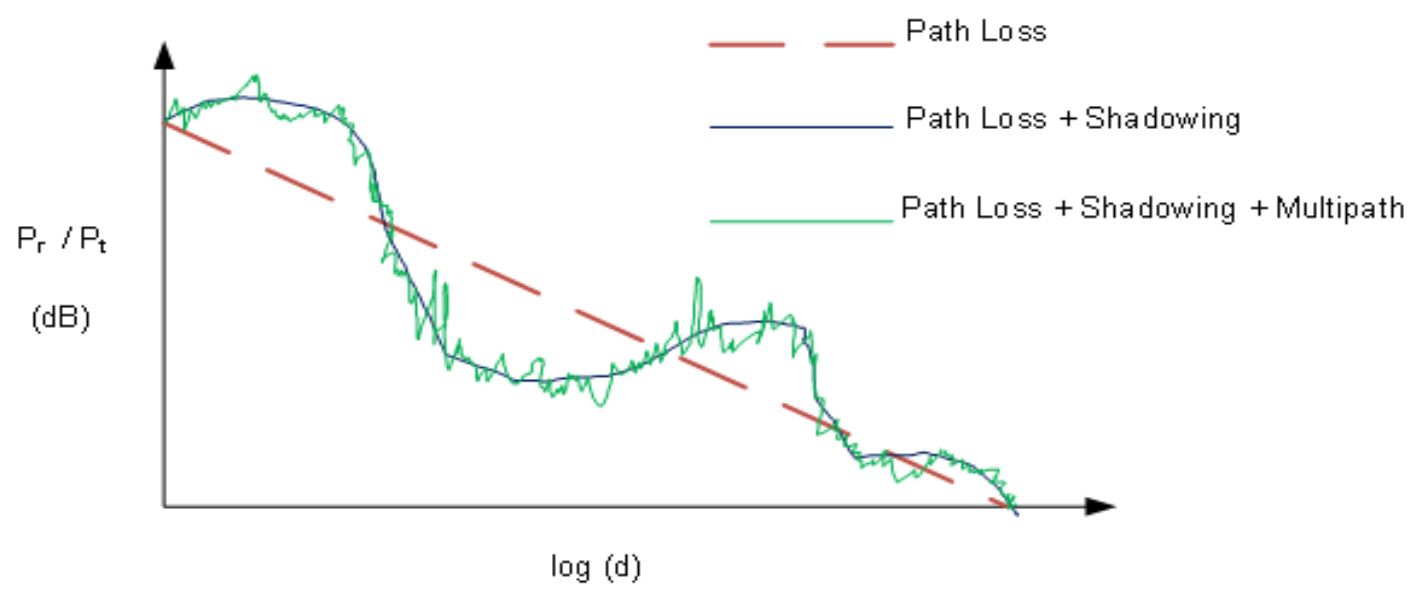

Figure 2.1: Effects of path loss, shadowing and multipath on $P_{r} / P_{t}(\mathrm{~dB})$ ratio with $\log$ distance [18].

Free-space path loss model is used when the signal propagates along a straight line without any obstruction between the transmitter and receiver [18]. In addition to free path loss model, empirical path loss models are developed to estimate the path loss in the typical wireless communication environments. Empirical path loss models (Okumara [18], Hata [18], COST 231 [16,18], SUI [19], LTE $[20,21]$, etc.) are based on actual measurements. Considering simplified path loss model, the combined effect of path loss and shadowing can be modelled in linear form as follows [18]

$$
P_{r}=P_{t} \bar{K}\left(\frac{d_{0}}{d}\right)^{a} \psi
$$

where $a$ is the path loss exponent. The values of $a$ can be obtained to approximate either an analytical or empirical model [18]. The values are normally in range of 2 to 4 , where 2 is for free-space propagation and 4 is for relatively lossy environment. $\bar{K}$ is a unitless constant that depends on the antenna characteristics and free -space path loss at distance $d_{0}$. Typical value for $d_{0}$ is $1 \mathrm{~m}$ for indoor and $100 \mathrm{~m}$ for outdoor environments [18]. The shadowing is modelled as a log-normal random variable, given by $\psi=10^{\left(\tilde{\eta} \sigma_{S F} / 10\right)}$, where $\sigma_{S F}$ is the shadowing standard deviation in $\mathrm{dB}$ and $\tilde{\eta}$ is a zero mean Gaussian random variable 
with unit variance. The distance $d$ represents the distance of the receiver from the transmitter.

Fading wireless channels can be categorised in two ways. The first type of classification discusses whether the fading is flat (frequency non-selective) or frequency selective, while the second classification is based on the rate at which the wireless channel is changing, ie. slow fading or fast fading. In this thesis, we only consider flat fading and slow fading channels.

\subsection{Multiple Antenna Systems}

Multiple antennas at the transmitter and the receiver have been shown to improve capacity, quality and reliability of the wireless systems [22]. Such systems are known as MIMO systems. In the literature there has been vast research on MIMO, which was pioneered by Winters [23], Teletar [24] and Foschini and Gans [25].

Under suitable channel fading conditions, having multiple antennas at transmitters and receivers provides an additional spatial dimension [17], which can be exploited by spatially multiplexing several data streams onto the MIMO channel. Employing multiple antennas can also provide dimensions to separate the desired and undesired signals, which we will discuss in the following chapters. In addition to the MIMO antenna configuration, there are two other types of antenna configurations: single-input multiple-output (SIMO) and multipleinput single-output (MISO). SIMO has a single transmit antenna and multiple receive antennas (receiver diversity only), whereas MISO has multiple transmit antennas and a single receive antenna (transmitter diversity only).

\subsubsection{Single User Systems}

Single User (SU) systems are the systems where a transmitter serves only one user, therefore each transmitter is dedicated to a user. Transmit beamforming techniques [26-30] with CSI at the transmitter, are shown to improve the performance of the system in fading channels, by using complex weights at transmit antennas [31]. The beamforming improves the SNR and also achieves higher array gains with perfect CSI at the transmitter. However, these gains are shown to be degraded if the channel estimation at the transmitters is erroneous [28,31].

\subsubsection{Multiuser Systems}

Multiuser (MU) systems are the systems where a transmitter serves to multiple users. In order to accommodate multiple users, multiple antennas can be used. Advancements in LTE standards will enable multiuser communication, 
where the transmitter or BS uses multiple antennas to serve multiple users in the downlink [32]. Precoding is an important component in MU systems as it allows spatial multiplexing of multiple users [33]. There are numerous linear and non-linear precoding strategies developed to improve the performance of MU systems. Interference is one of the reasons that reduce the throughput of MU systems. Precoding schemes are key to mitigate the interference caused by the existence of multiple users in the same system. Furthermore, CSI at the BS is critical for precoding in MU systems. Like SU systems, MU systems also suffer the imperfect channel estimations, because, with imperfect CSI, the precoding scheme cannot mitigate interference effectively [34,35].

\subsection{Cellular Networks}

The basic premise behind cellular system design is frequency reuse [18]. Communication within a cell is controlled by a centralised BS. Ideally the coverage area of a cellular network is divided into partially overlapping cells. Due to path loss and shadowing in particular, the power from the BS "leaks" to the other users in adjacent cells [36-38]. This type of interference is called intercell interference. The BSs or the users in adjacent cells can cooperate in order to mitigate intercell interference [39]. In addition to intercell interference, the users also suffer the interference caused by other users within the same cell. This type of interference is called intracell interference. There are many ways to deal with both intracell and intercell interference in the literature, which will discuss in later in this chapter.

In this thesis, we will study following operating modes of cellular networks

- Uplink: The communication is from the users to the BS.

- Downlink: The communication is from the BS to the users.

Table 2.1: Environment parameters for simulations

\begin{tabular}{|l|l|}
\hline Parameter & Value \\
\hline BS Height $(\mathrm{m})$ & 32 \\
\hline MS height $(\mathrm{m})$ & 1.5 \\
\hline Carrier frequency $f_{c}$ & $1900 \mathrm{MHz}$ \\
\hline Log-normal shadowing standard deviation $(\mathrm{dB})$ & 8 \\
\hline
\end{tabular}

In the next section, we will define the cellular scenarios that we use in this thesis in detail. In these scenarios, the 3GPP parameters given in Table 2.1 are considered. We note that intersite distance is $2 \mathrm{~km}$ for urban users and $4 \mathrm{~km}$ for suburban users. Transmitter power is assumed to be $23 \mathrm{dBm}$ and $46 \mathrm{dBm}$ for 


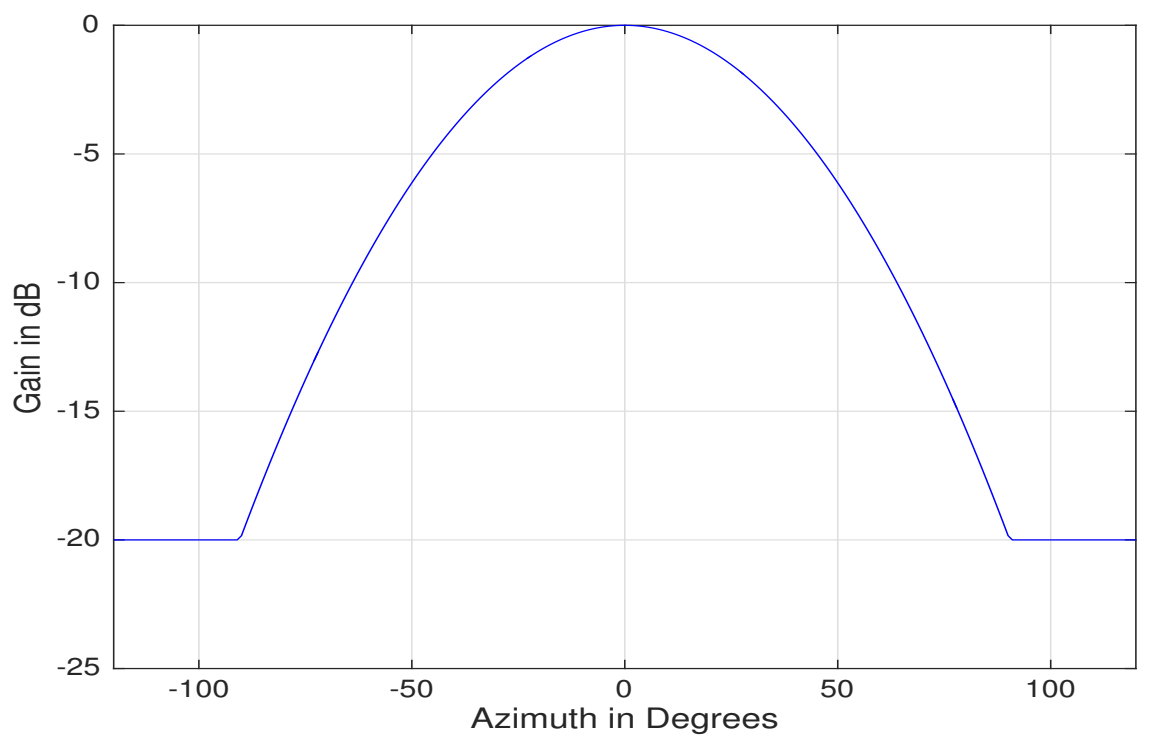

Figure 2.2: Antenna pattern for 3-sector cells

uplink and downlink, respectively. The simulations are performed in a 19-cell setting given in Fig. 2.3 with an urban macrocell pathloss model which is based on the modified COST231 Hata urban propagation model given in [16] such that

$$
\begin{aligned}
\mathrm{PL}_{[d B]} & =\left(44.9-6.55 \log _{10}\left(h_{\mathrm{BS}}\right)\right) \log _{10}\left(\frac{d}{1000}\right)+45.5+\left(35.46-1.1 h_{\mathrm{MS}}\right) \log _{10}\left(f_{c}\right) \\
& \left.-13.82 \log _{10}\left(h_{\mathrm{BS}}+0.7 h_{\mathrm{MS}}\right)+C\right)
\end{aligned}
$$

where $h_{\mathrm{BS}}$ is the BS antenna height in meters, $h_{\mathrm{MS}}$ is the mobile station (MS) ${ }^{1}$ antenna height, $f_{c}$ is the carrier frequency in $\mathrm{MHz}, d$ is the distance between the $\mathrm{BS}$ and MS in meters, $C$ is a constant factor $(0 \mathrm{~dB}$ for suburban and $3 \mathrm{~dB}$ for urban macro) [16]. We also consider the 3 -sector antenna pattern given in [16] and is plotted in Fig. 2.2.

The 3 -sector antenna pattern in Fig. 2.2 is specified by $A(\theta)=-\min \left[12\left(\frac{\theta}{\theta_{3 d B}}\right)^{2}, A_{m}\right]$, where $-180 \leq \theta \leq 180$. We also note that $A(\theta)$ is the gain with resptect to $\theta$ which is defined as the angle between the direction of interest and boresight of the antenna, $\theta_{3 \mathrm{~dB}}$ is the $3 \mathrm{~dB}$ beam-width in degrees and specified as 70 degrees for 3 sector scenario in [16]. $A_{m}$ is the maximum attenuation and $A_{m}=20 \mathrm{~dB}$ for 3 sector scenario. Antenna boresight directions are given as red, green and blue lines for each cell in Fig. 2.3.

In the following section, we give the measurements of received SNR and SINR for various uplink and downlink scenarios.

\footnotetext{
${ }^{1}$ Please note that MS also refers to the user in this thesis.
} 


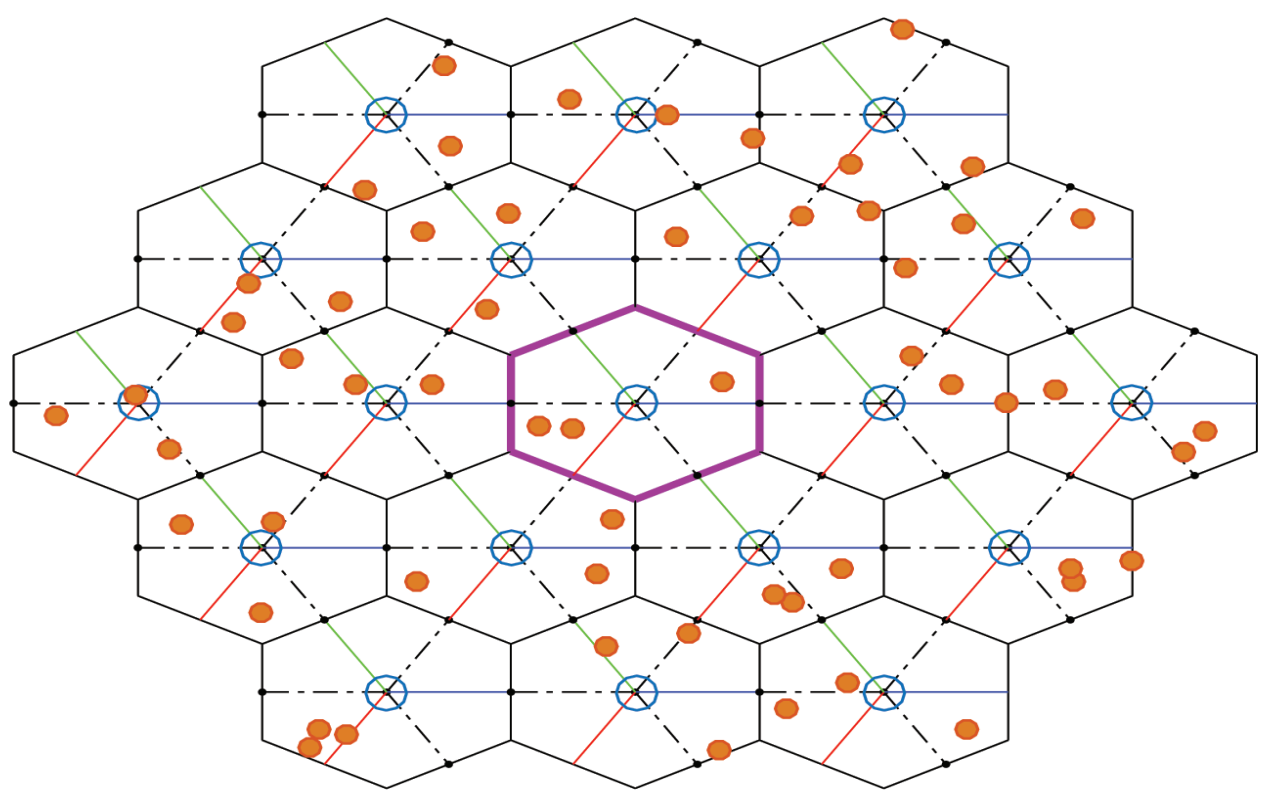

Figure 2.3: The setting used for cellular network simulations with 19 cells and 3 user in each cell. The snapshot was taken over 1 time-frame.

\subsubsection{Scenarios Considered for the Uplink}

We assume 2 uplink scenarios for our simulations based on user locations.

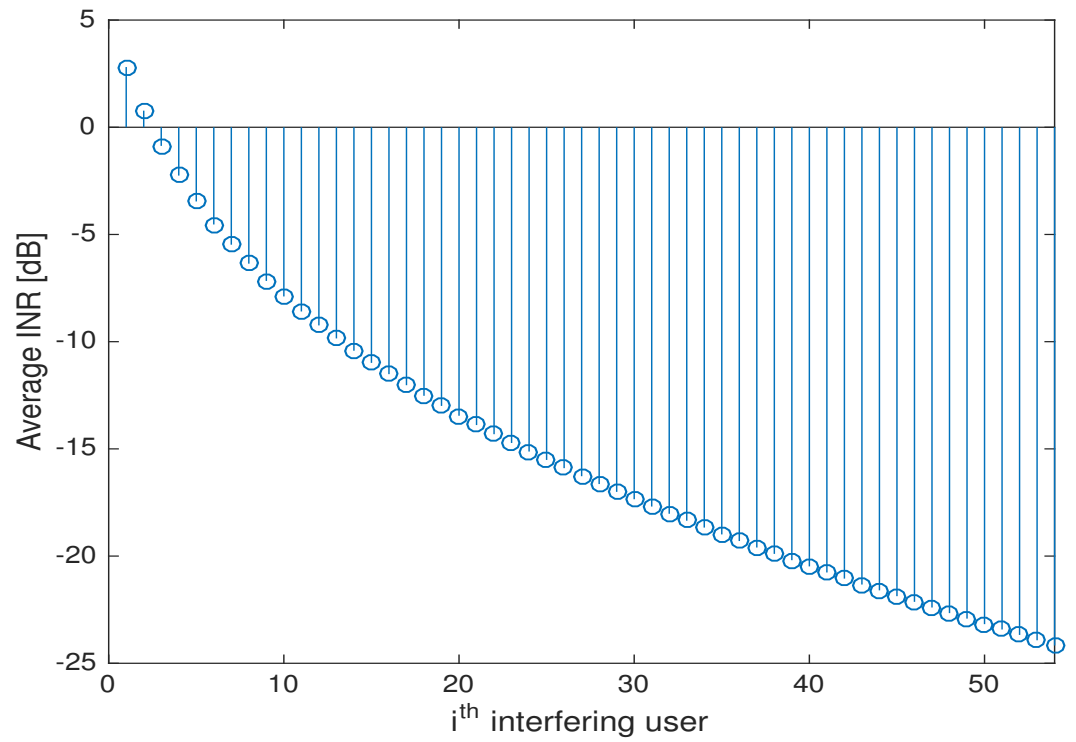

Figure 2.4: Average INR for each interfering user in the Uplink

\section{Scenario 1: Random User Locations}

We first assume that 3 users are located randomly within each cell as in Fig. 2.3. In Fig. 2.4, we show the average INR for each interfering user. As seen inFig. 2.4 , the first 2 interfering users are significantly stronger than other interferers. 
In Fig. 2.5, we show the cdf of SNR and SINR from a user to the BS. In order to analyse the impact of intercell interference, we also calculate SINR by removing some strongest intercell interference. In Fig. 2.5, Scenario A represents the SINR when we remove the strongest intercell interference from 3 users in an adjacent cell. Scenario B indicates the SINR without the strongest intercell interference from 6 users. Finally Scenario C denotes that the strongest intercell interference from 9 users is removed.

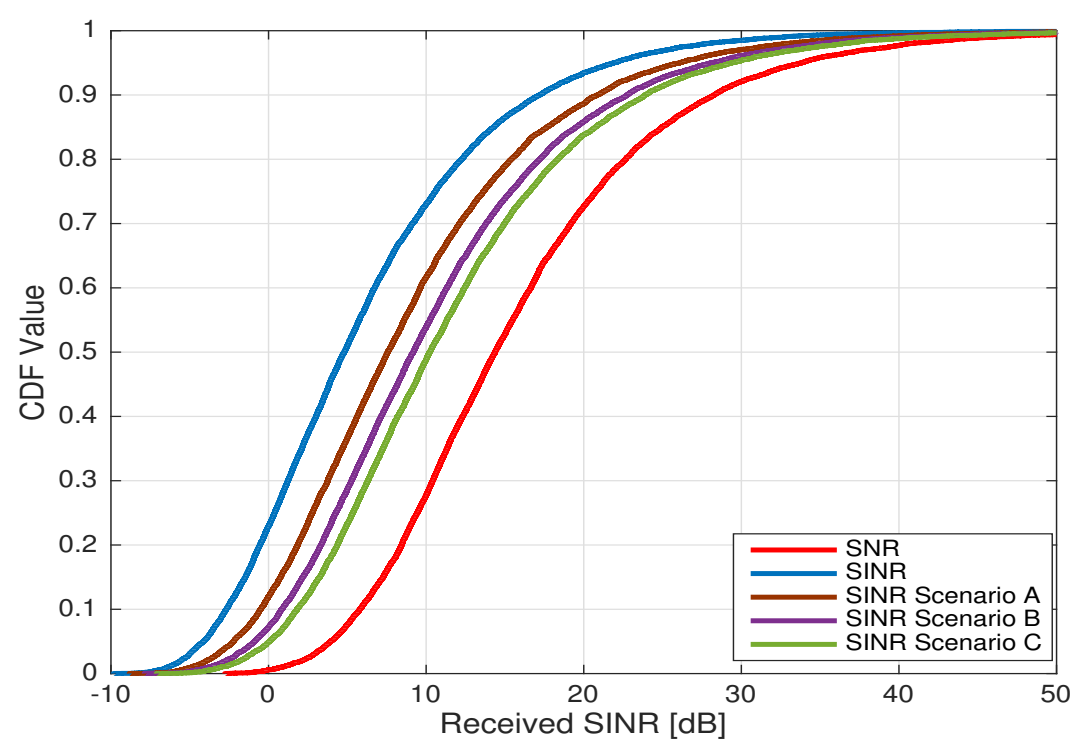

Figure 2.5: Received SINR for urban random user locations in uplink cellular networks

In Fig. 2.5, we note that cancelling interference from the strongest cell provide significant gain. Especially as in Scenario C, mitigating intercell interference from strongest 3 cells, achieves values close to SNR results. This indicates that after removing interference from 3 cells, the remaining interference power is low. Based on simulation results, if we assume dominant interference is from adjacent 3 cells ( 9 users in total, Scenario $\mathrm{C}$ ), $\gamma=\frac{\mathrm{INR}_{\mathrm{rem}}}{\mathrm{INR}_{\mathrm{dom}}} \approx 0.1424$. The $\gamma$ ratio will be used as a system parameter throughout this thesis, dependent on the ratio of dominant over remaining interference-noise-ratio ${ }^{2}$ (INR). Considering only 3 users as dominant interference (Scenario A), $\frac{\mathrm{INR}_{\text {rem }}}{\mathrm{INR}_{\mathrm{dom}}} \approx 0.3189$, where as considering 6 users as dominant interference(Scenario B), $\frac{\mathrm{INR}_{\mathrm{rem}}}{\mathrm{INR}_{\mathrm{dom}}} \approx 0.2043$.

\section{Scenario 2: Cell-Edge User Locations}

In this scenario, we assume that the users are at cell-edge. These users are assumed to form the lowest $5 \%$ of the CDF of SNR for randomly located users

\footnotetext{
${ }^{2} \mathrm{INR}_{\text {rem }}$ is the remaining interference to noise ratio, $\mathrm{INR}_{\mathrm{dom}}$ is the dominant interference to noise ratio.
} 


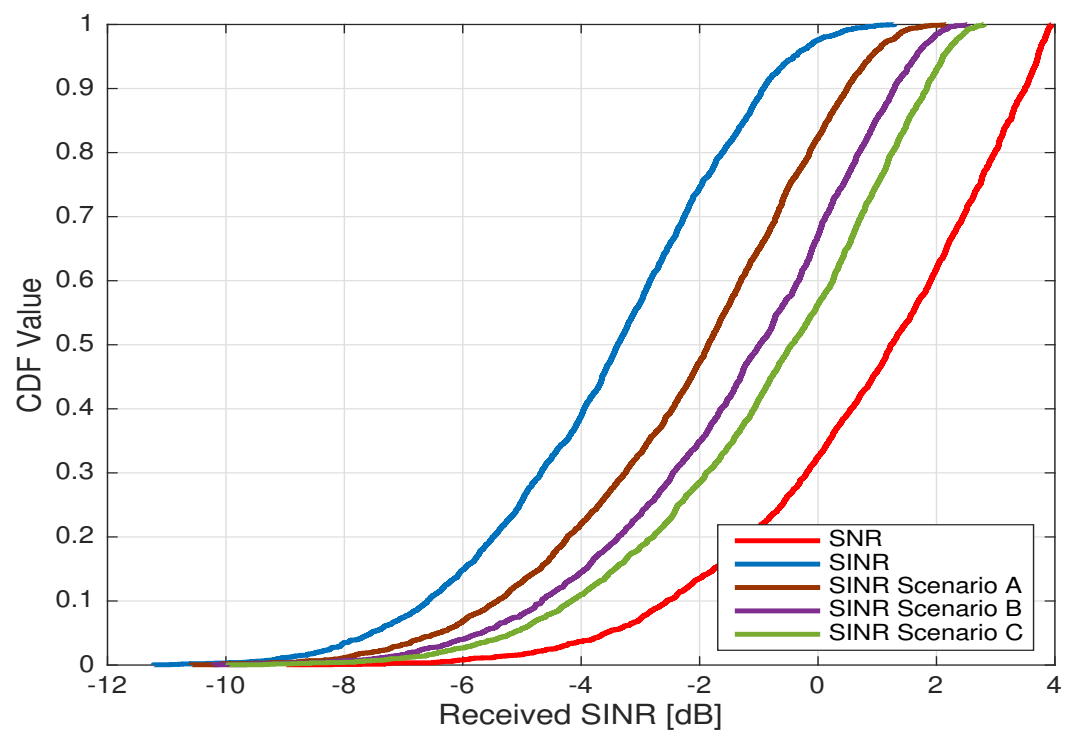

Figure 2.6: Received SINR for urban cell-edge user locations in uplink cellular networks

in Fig. 2.5. Using the simulation results in Fig. 2.5, we define the cell-edge users which have SNR less than $3.95 \mathrm{~dB}$. Then we evaluate the simulations for these users to find their SINRs as given in Fig. 2.6.

Similar to random locations, we analyse the results for SNR, SINR and also SINR for Scenario A, B and C which were mentioned in previous section.

In Fig. 2.6, it is observed that cancelling interference from the strongest cell provide significant gain. However, it is also shown that remaining interference for cell edge users is more significant compared to random users. Based on simulation results, for Scenario A, we find $\gamma=\frac{\mathrm{INR}_{\mathrm{rem}}}{\mathrm{INR}_{\mathrm{dom}}} \approx 0.95$. For Scenario B, $\frac{\mathrm{INR}_{\text {rem }}}{\mathrm{INR}_{\text {dom }}} \approx 0.63$, and for Scenario $\mathrm{C}, \frac{\mathrm{INR}_{\text {rem }}}{\mathrm{INR}_{\mathrm{dom}}} \approx 0.44$. As we consider remaining interference is the interference that we have limited knowledge about, higher remaining interference refers to a significant limiting factor in a cellular network.

\subsubsection{Scenarios Considered for the Downlink}

In downlink, we assume our scenarios as urban and suburban. We also analyse user locations as random user locations and cell-edge user locations in urban environments.

\section{Urban Areas}

Scenario 1 - Random User Locations: First, we assume that the users are located randomly in an urban environment. In Fig. 2.7, we show average INR for each interfering BS. As seen in Fig. 2.7, the first 2 interferers are significantly stronger than other interferers. 


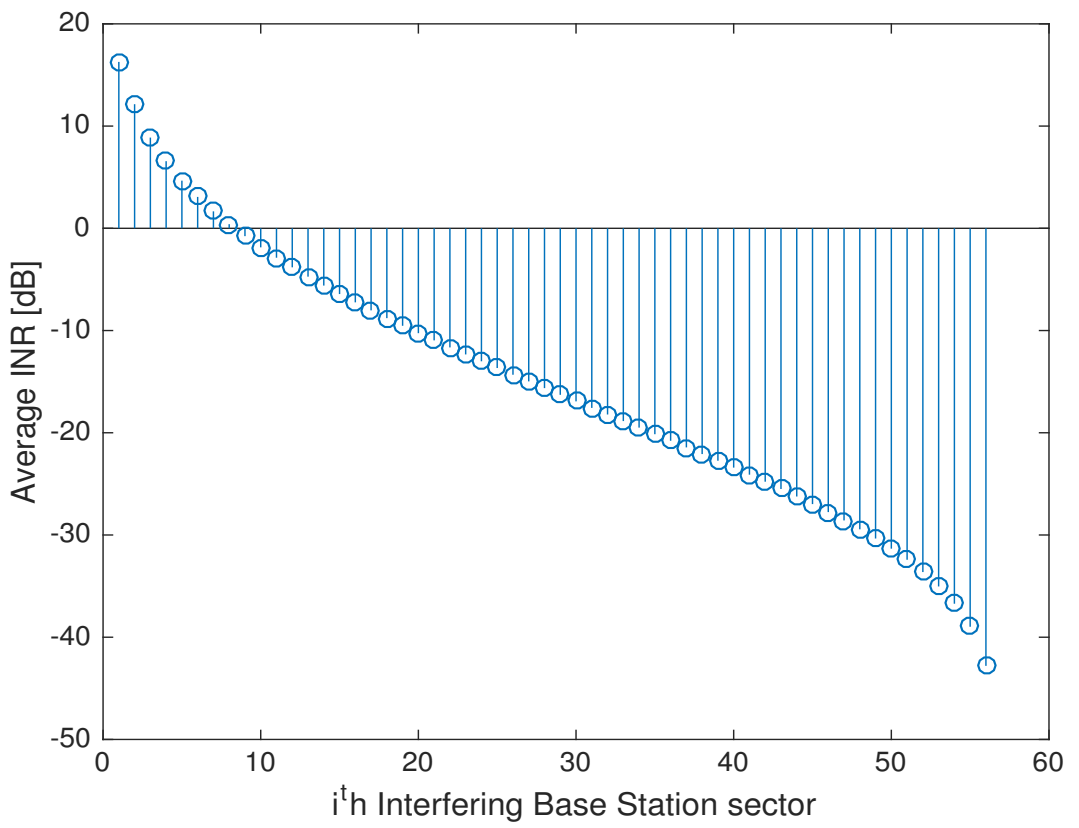

Figure 2.7: Average INR for each interfering user in the Downlink

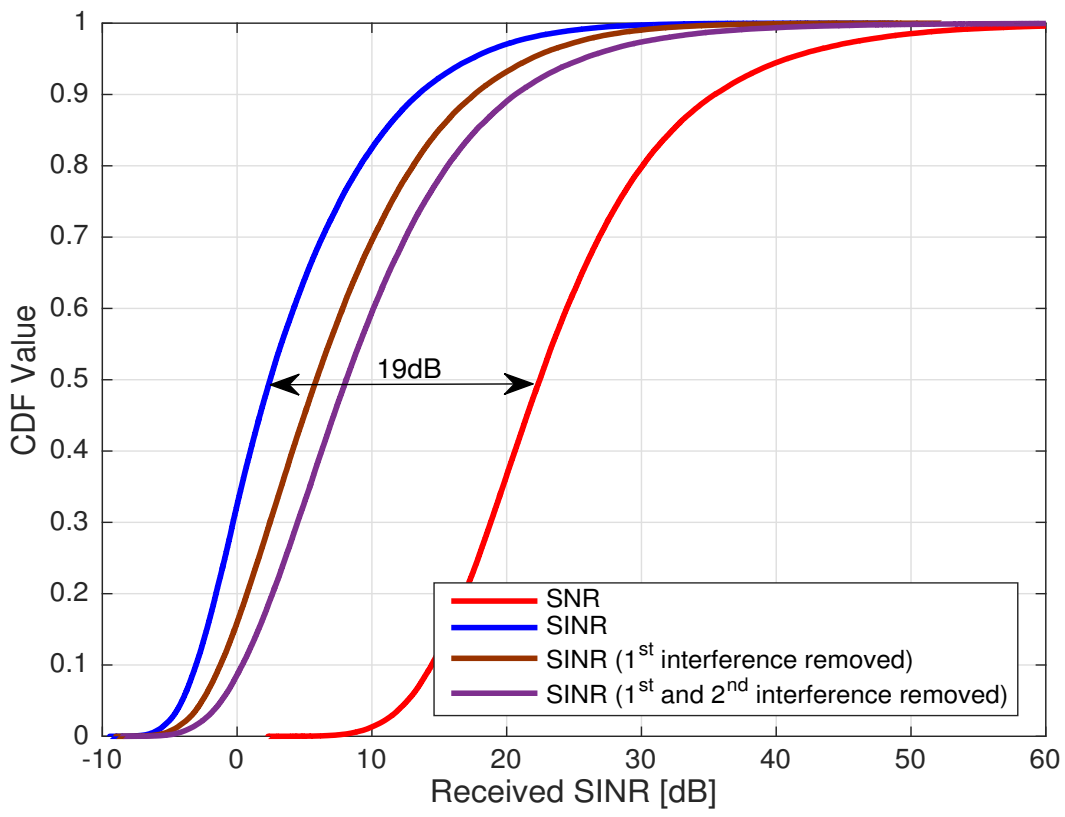

Figure 2.8: Received SINR for urban random user locations in downlink cellular networks

In Fig. 2.8, we give SNR and SINR results. We also calculate the SINR by removing the strongest two interferers, to show the gain that can be achieved by intercell interference cancellation. It can be seen in Fig. 2.8 that the total interference causes a $19 \mathrm{~dB}$ loss at the median cdf point. If we cancel the 
strongest two interferers, this loss decreases to approximately $12 \mathrm{~dB}$. The uncancelled interferers result in remaining interference. Considering the existence of 2 dominant interferers, we find $\gamma=\frac{\mathrm{INR}_{\mathrm{rem}}}{\mathrm{INR}_{\mathrm{dom}}} \approx 0.68$ on average for randomly located users. However, if we consider 1 dominant interference and the rest as remaining interference, $\frac{\mathrm{INR}_{\mathrm{rem}}}{\mathrm{INR}_{\mathrm{dom}}} \approx 1.65$, which is significantly high.

Scenario 2 - Cell-Edge User Locations: In this scenario, we assume that the users are in cell-edge locations which form the lowest $5 \%$ of the CDF of received SNR for randomly located users in Fig. 2.8. Using the simulation results in Fig. 2.8, we define cell-edge users as those which have received SNR less than $12.5 \mathrm{~dB}$. Then we evaluate the simulations for these users to find their SINRs as given in Fig. 2.9. Here, we see that the total interference causes a 15.5 $\mathrm{dB}$ loss at the median cdf point. If we cancel the first two interference terms, this loss decreases to approximately $13 \mathrm{~dB}$ which is attributed to the SNR loss caused by the remaining interference. If we consider the existence of 2 dominant interferers, we find $\gamma=\frac{\mathrm{INR}_{\text {rem }}}{\mathrm{INR}_{\mathrm{dom}}} \approx 1.49$ on average for cell-edge users. However, if we consider 1 dominant interference and the rest as remaining interference, $\frac{\mathrm{INR}_{\text {rem }}}{\mathrm{INR}_{\mathrm{dom}}} \approx 3.05$, which is a very high ratio.

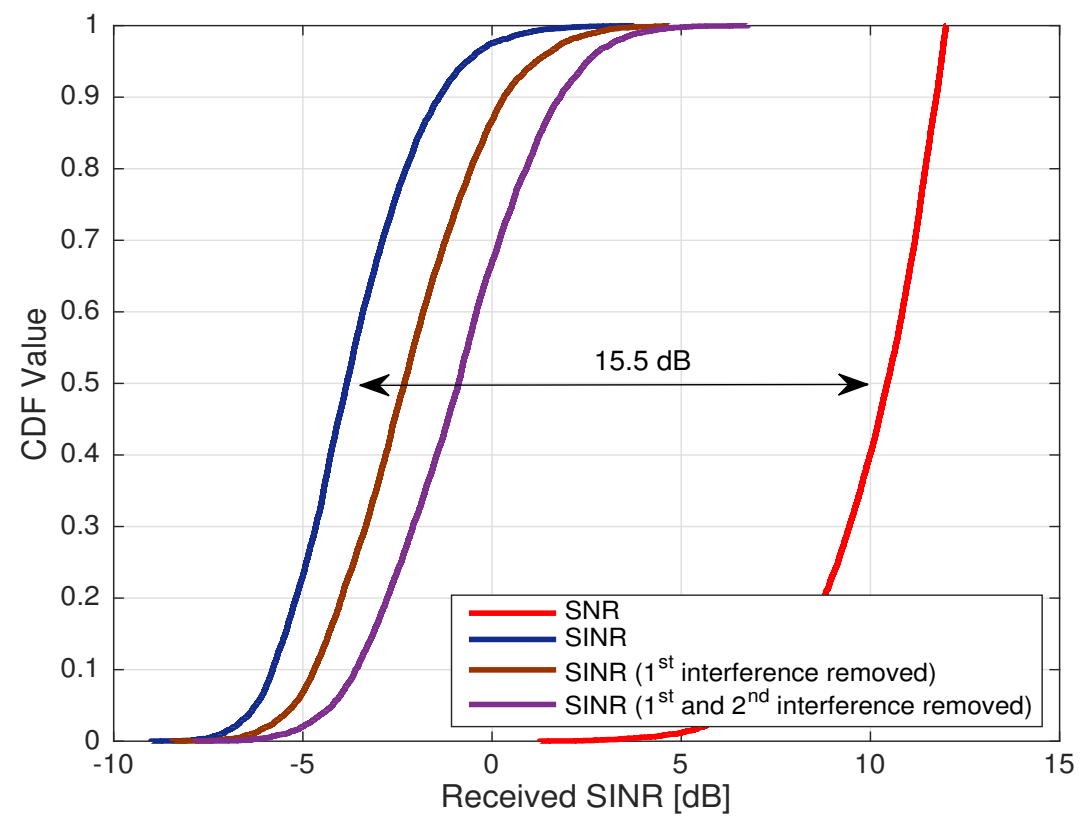

Figure 2.9: Received SINR for urban cell-edge user locations in downlink celluar systems

\section{Suburban Areas}

The path loss for suburban areas is given in [40], which is $3 \mathrm{~dB}$ less than the corresponding path loss for urban users. Assuming that the intersite distance is $4 \mathrm{~km}$, we calculate SINR for suburban areas and the results are given in Fig. 


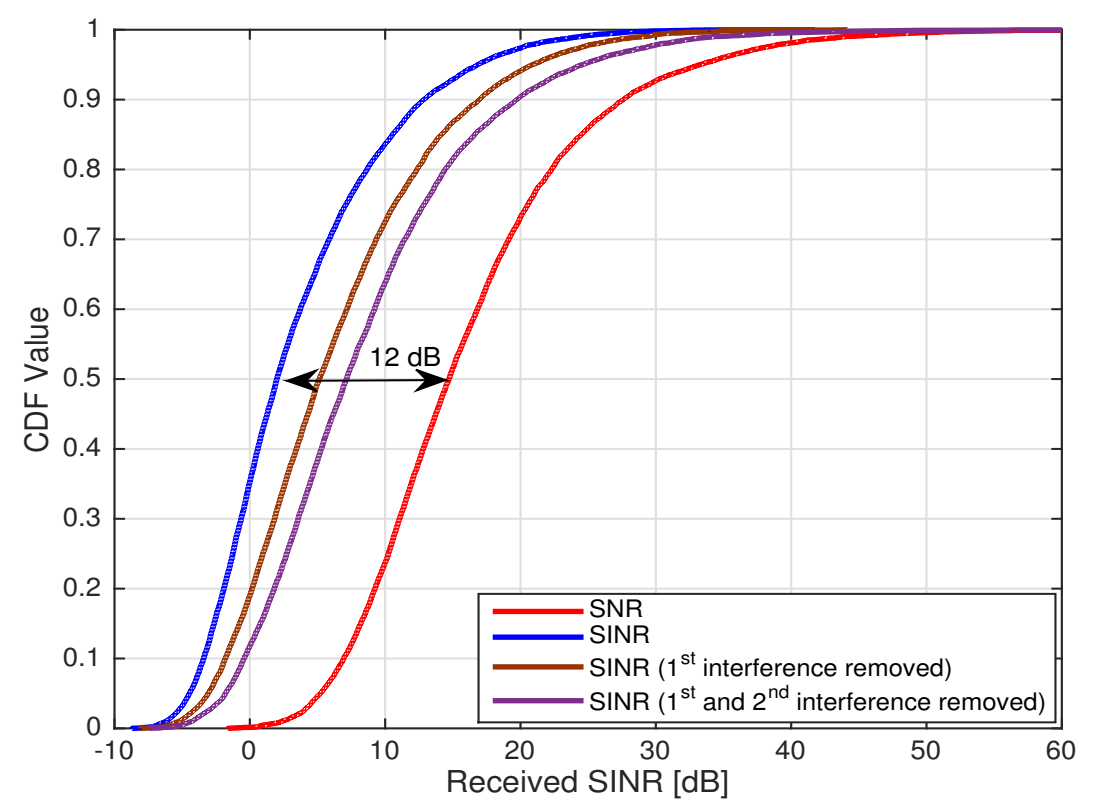

Figure 2.10: Received SINR for suburban users in downlink cellular networks

\subsection{0 .}

As seen in Fig. 2.10, there is approximately a $12 \mathrm{~dB}$ loss at the median cdf point, caused by interference. This loss descreases to approximately $5 \mathrm{~dB}$ if we can cancel the 2 strongest interferers. Even though the loss caused by interference is less than the corresponding value for urban users, the SINR of suburban users is similar to that of the urban users due to the increased path loss caused by a longer intersite distance. Considering the existence of 2 dominant interferers, we find $\gamma=\frac{\mathrm{INR}_{\text {rem }}}{\mathrm{INR}_{\text {dom }}} \approx 0.62$ on average for randomly located users in suburban environments. If we consider 1 dominant interference, then $\gamma=\frac{\mathrm{INR}_{\text {rem }}}{\mathrm{INR}_{\mathrm{dom}}} \approx 1.51$.

\subsection{Interference Management Systems}

The simulation results given in previous section for both uplink and downlink systems indicate that cellular networks suffer from interference significantly. In order to provide high-quality transmission to the users of these systems, cancelling interference has a crucial importance on the design of wireless systems [17].

\subsubsection{Traditional Interference Management Systems}

Here, we summarize some of the interference management approaches used in practice as follows

- Decode: If interference is strong, then the interference signal can be 
decoded along with the desired signal. This is however a very intricate approach as it requires perfect knowledge of the interfering channel as well as other information such as precoding vectors. This approach is less common due to its complexity, although it is supported by $[39,41,42]$.

- Treat as noise: If interference is weak, then the interfering signal is treated as noise, [43-46]. However, as shown in the scenarios of Section 2.3, the interference levels in commonly used cellular networks are not weak enough to be treated as noise. Therefore more sophisticated approaches are needed to deal with strong interference.

- Orthogonalise: This is the basis for frequency (or time) division multiple access. In other words, spectrum is divided in a cake-cutting fashion [11]. This is a very popular approach in practice, however it is problematic as it doesn't provide a solution to use the spectrum very efficiently. These approaches can be used to divide the resources among the users such that each user gets a fraction and sum of these fractions is equal to 1 [11]. In the following sections, we will study a new approach, interference alignment (IA), which proposes a solution of more efficient usage of the spectrum.

- Other methods: There are also many other methods commonly used in practical systems such as frequency re-use patterns [47] which is based on adjacent cells using different frequencies to ensure that the mutual interference between users remains below a harmful level $[48,49]$. The beamformers are used to produce an array response with a high gain in the direction of the signal of interest while attenuating noise and interference arriving from all other directions [48]. The other well known methods are soft cancellation [50], space-time equalisation [51] and turbo methods [52].

\subsection{Interference Alignment - The Concept}

In this section, we give the main idea of the IA, which is originated from the linear algebra. However, it is not very easy to implement IA because of many disruptive challenges we encounter during implementation. Consider a system of linear equations such that [9]

$$
\begin{aligned}
& y_{1}=h_{11} x_{1}+h_{12} x_{2}+\ldots+h_{1 K} x_{K} \\
& y_{2}=h_{21} x_{1}+h_{22} x_{2}+\ldots+h_{2 K} x_{K} \\
& \ldots \\
& y_{B}=h_{B 1} x_{1}+h_{B 2} x_{2}+\ldots+h_{B K} x_{K},
\end{aligned}
$$

where we have $B$ received signals $y_{1}, y_{2}, \ldots y_{B}$ which are linear combinations of $K$ 


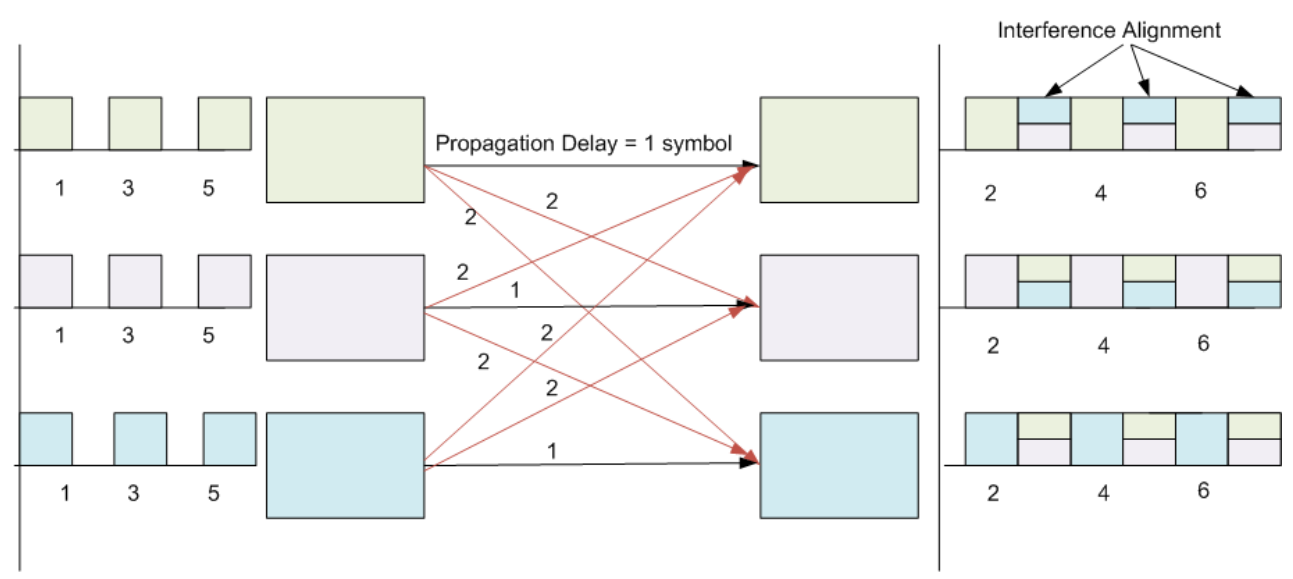

Figure 2.11: Interference alignment - Toy example of [9]

symbols with channel coefficients $h_{i j}$. In the parlance of interference networks, we assume $K$ to be the number of transmitters and $B$ to be the bandwidth or signalling dimensions. Since the channel is linear, each signalling dimension produces a linear combination of the transmitted information symbols. Thus, a receiver has access to $B$ signalling dimensions. An important question which should be asked in this stage is, how many signalling dimensions (or bandwidth) are needed for the receiver to be able to resolve its desired symbol from the remaining $K-1$ undesired symbols?

Before IA, a total of $K$ signalling dimensions (or a bandwidth of $K$ ) are used so that each receiver is able to resolve its desired one dimensional signal. In the terminology of wireless interference networks, this solution corresponds to the cake cutting interpretation of spectrum allocation, i.e., the total bandwidth is divided among the $K$ users much like a cake so that each user gets $1 / K$ th of the resources.

Earlier in Chapter 1, we gave an example of $K$ speaker-listener pairs in order to explain what IA provides. IA provides $1 / 2$ of the total available time interference free to all pairs, instead of $1 / K$. This huge gain may seem impossible at first. This seemingly impossible result is made possible by the concept of IA which can be made in time, frequency or space. Let us consider $K$ user interference channel where there is a propagation delay from transmitter $i$ to receiver $j$, which is denoted as $T_{i j}$ [9]. Suppose the locations of the transmitters and receivers can be configured such that the delay $T_{i i}$ from each transmitter to the intended receiver is an even multiple of a basic symbol duration $T_{s}$, while the signal propagation delays $T_{i j},(i \neq j)$ from each transmitter to all unintended receivers are odd multiples of symbol duration. The communication strategy is as seen in the Figure 2.11, such that all transmissions occur simultaneously at even symbol durations. Note that with this policy, each receiver sees its own transmitter's signal interference-free over even time periods, while it sees all interference signals simultaneously over odd time periods. That is why, each 
user is able to achieve $1 / 2$ degrees of freedom ${ }^{3}$ and the total degrees of freedom achieved is equal to $K / 2$. This result has special importance in cellular and ad hoc networks, showing that coordination between users can help overcome the limiting effects of interference generated by simultaneous transmission.

The earliest application of IA, even though it was not called IA at that time, was mentioned $[53,54]$ in the context of an index coding problem. As described by the authors of these papers, this setting can be seen as a wireless broadcast channel where users have information on the transmitted signals. They mention that not all transmitted signals are desired by all nodes, which is considered as the key idea to IA, allowing undesired transmitted signals to be consolidated into a smaller dimension.

\section{Interference Alignment with Precoding Vectors}

Precoding can be defined as signal processing to consolidate signals into dimensions via vectors that are multiplied with signals at transmitters. These precoders are generated in order to align all interference in a subspace spanned by all undesired signals. This subspace is called the interference subspace [15]. In Fig. 2.12, we give an example of IA with precoding vectors, where there are four transmitters and four receivers. Each transmitter $i$ sends their signal with a precoding vector $\mathbf{v}_{i}$. Each signal is then delivered to each receiver $j$ through a wireless channel $\mathbf{H}_{i j}$. Each receiver also sees interference from undesired transmitters. As seen in this figure, all undesired interference is aligned within a subspace which is coloured as tinted grey. The condition for perfect alignment within the interference subspace is the fact that the interference subspace should be spanned by all interfering signals. The definition of span can be given as follows

- In linear algebra, span of a set of vectors in a vector space is the intersection of all subspaces containing that set. The linear span of a set of vectors is therefore a vector space [55].

In some networks (i.e. cellular networks), the precoding vectors are aligned in the null space of the unintended receivers. Thus, their leakage to the unintended receiver could be zero. The definition of null space can be given as follows

- In linear algebra, null space of a matrix $\mathbf{A}$ is the set of solutions of $\mathbf{x}$ to the equation $\mathbf{A x}=\mathbf{0}$, where $\mathbf{0}$ is the zero vector. [55]. In other words, any row vector of $\mathbf{A}$ can be used to null $\mathbf{x}$.

In the following sections, we give some examples of IA applications for a better understanding of how to align interference using precoding vectors.

\footnotetext{
${ }^{3}$ The degrees of freedom is an approximation of sum capacity which is commonly used in IA literature. The derivation of the degrees of freedom is given in [9].
} 


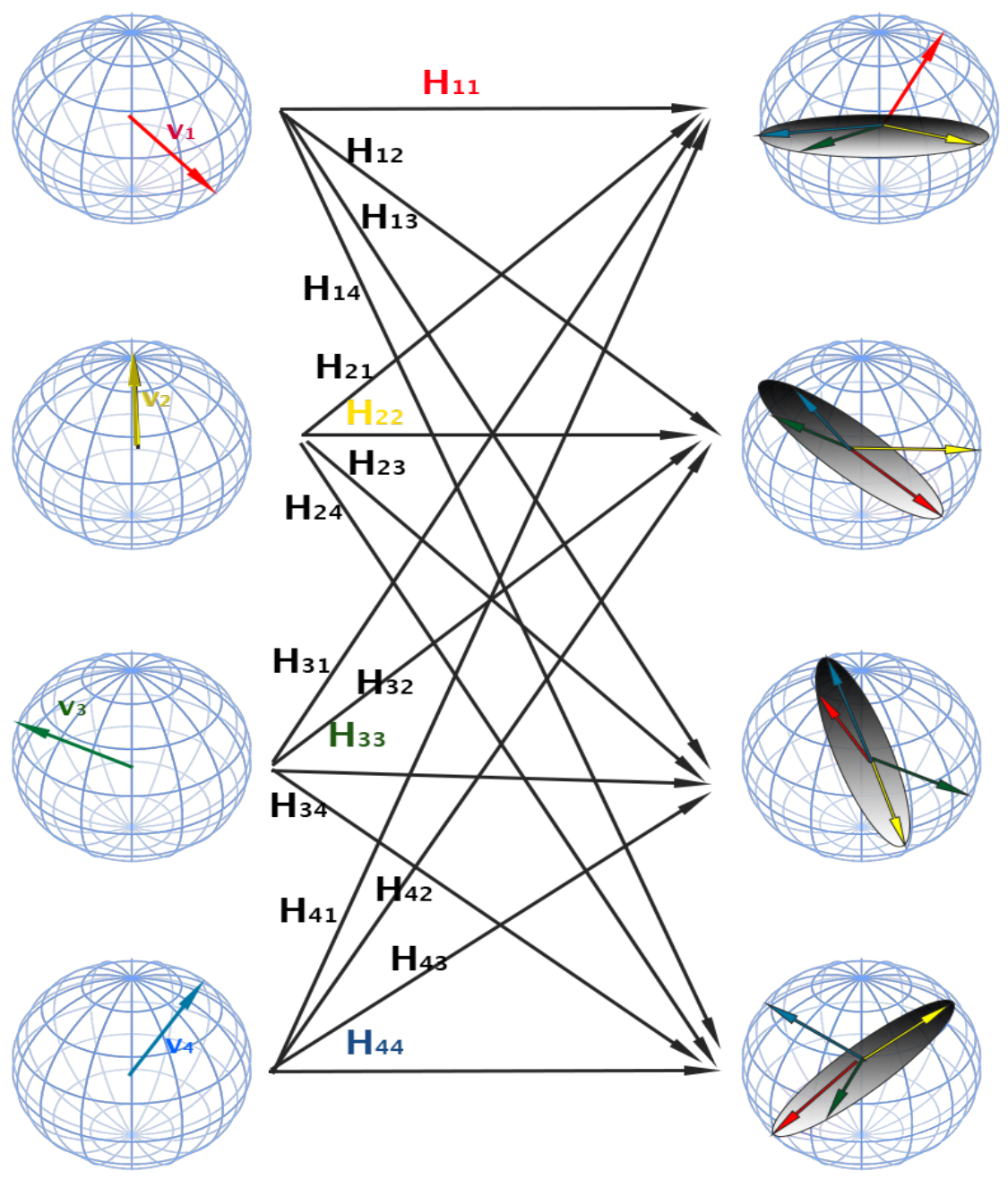

Figure 2.12: Interference alignment with precoding vectors [15]

\subsection{Interference Alignment in MIMO X Chan- nels}

\subsubsection{System Model}

In this section, we present an application of IA in a MIMO X channel of [14], which is a system with two transmitters each equipped with $N_{t}$ antennas and two receivers, each equipped with $N_{r}$ antennas. Each transmitter transmits a message for each receiver, therefore there are four independent messages in the channel. The MIMO X channel is shown in Fig. 2.13.

We can also describe the system with the following system equation

$$
\begin{aligned}
& \mathbf{y}_{1}=\mathbf{H}_{11}\left(\mathbf{v}_{11} x_{11}+\mathbf{v}_{12} x_{12}\right)+\mathbf{G}_{21}\left(\mathbf{v}_{21} x_{21}+\mathbf{v}_{22} x_{22}\right)+\mathbf{n}, \\
& \mathbf{y}_{2}=\mathbf{H}_{22}\left(\mathbf{v}_{21} x_{21}+\mathbf{v}_{22} x_{22}\right)+\mathbf{G}_{12}\left(\mathbf{v}_{11} x_{11}+\mathbf{v}_{12} x_{12}\right)+\mathbf{n},
\end{aligned}
$$


where $\mathbf{y}_{1}$ is the $N_{r} \times 1$ received signal at receiver 1 . $\mathbf{y}_{2}$ is the $\left(N_{r} \times 1\right)$ received vector at receiver 2 . Transmitter 1 transmits the message $x_{11}$, which is desired for receiver 1 with the $\left(N_{t} \times 1\right)$ precoding vector $\mathbf{v}_{11}$. Similarly, it tranmsits the message $x_{12}$, which is desired for receiver 2 with the $\left(N_{t} \times 1\right)$ precoding vector $\mathbf{v}_{12}$. Transmitter 2 transmits the message $x_{21}$ which is desired for receiver 1 , with the $\left(N_{t} \times 1\right)$ precoding vector $\mathbf{v}_{21}$. Besides, it transmits the message $x_{22}$ which is desired for receiver 2 with the $\left(N_{t} \times 1\right)$ precoding vector $\mathbf{v}_{22}$. The transmitted signals from transmitter 1 are received by receiver 1 through an $\left(N_{r} \times N_{t}\right)$ channel $\mathbf{H}_{11}$, while the transmitted signals from transmitter 2 are received by receiver 1 through a $\left(N_{r} \times N_{t}\right)$ channel $\mathbf{G}_{21}$. Similarly receiver 2 receives signals from transmitter 1 and 2 through $\left(N_{r} \times N_{t}\right)$ channels $\mathbf{G}_{12}$ and $\mathbf{H}_{22}$, respectively. As in [14], we assume that the channel matrices are generated from a continuous probability distribution, so that, almost surely channel matrices will have an equal rank to the minimum number of it rows and columns [14]. $\mathbf{n}$ is denoted as $\left(N_{r} \times 1\right)$ additive white Gaussian noise (AWGN) vector.

IA is achieved with precoding vectors by aligning undesired messages along a one-dimensional vector. If we assume $N_{r}=N_{t}=3$, only three signalling dimensions are available. This is achieved by aligning the two undesired messages at each receiver along a vector, which is shown as red at receiver 1 , and blue at receiver 2 in Fig. 2.13. In order to find the precoding vectors, we first define a reference vector for messages desired at each receiver. We use $(3 \times 1)$ reference vectors $\boldsymbol{\eta}_{1}$ and $\boldsymbol{\eta}_{2}$ for the messages desired at receiver 1 and receiver 2 respectively. After selecting the reference vectors, we can define the precoding vectors which will align undesired signals

$$
\begin{aligned}
\mathbf{v}_{11} & =\mathbf{G}_{12}^{-1} \boldsymbol{\eta}_{1}, \\
\mathbf{v}_{12} & =\mathbf{H}_{11}^{-1} \boldsymbol{\eta}_{2}, \\
\mathbf{v}_{21} & =\mathbf{H}_{22}^{-1} \boldsymbol{\eta}_{1}, \\
\mathbf{v}_{22} & =\mathbf{G}_{21}^{-1} \boldsymbol{\eta}_{2} .
\end{aligned}
$$

After defining the precoding vectors, one could rewrite (2.4) and (2.5)

$$
\begin{aligned}
\mathbf{y}_{1} & =\mathbf{H}_{11}\left(\mathbf{G}_{12}^{-1} \boldsymbol{\eta}_{1} x_{1,1}+\mathbf{H}_{11}^{-1} \boldsymbol{\eta}_{2} x_{1,2}\right)+\mathbf{G}_{21}\left(\mathbf{H}_{22}^{-1} \boldsymbol{\eta}_{1} x_{2,1}+\mathbf{G}_{21}^{-1} \boldsymbol{\eta}_{2} x_{2,2}\right)+\mathbf{n}, \\
& =\mathbf{H}_{11} \mathbf{G}_{12}^{-1} \boldsymbol{\eta}_{1} x_{1,1}+\mathbf{G}_{21} \mathbf{H}_{22}^{-1} \boldsymbol{\eta}_{1} x_{2,1}+\boldsymbol{\eta}_{2}\left(x_{1,2}+x_{2,2}\right)+\mathbf{n},
\end{aligned}
$$

and

$$
\begin{aligned}
\mathbf{y}_{2} & =\mathbf{H}_{22}\left(\mathbf{H}_{22}^{-1} \boldsymbol{\eta}_{1} x_{2,1}+\mathbf{G}_{21}^{-1} \boldsymbol{\eta}_{2} x_{2,2}\right)+\mathbf{G}_{12}\left(\mathbf{G}_{12}^{-1} \boldsymbol{\eta}_{1} x_{1,1}+\mathbf{H}_{11}^{-1} \boldsymbol{\eta}_{2} x_{1,2}\right)+\mathbf{n}, \\
& =\mathbf{H}_{22} \mathbf{G}_{21}^{-1} \boldsymbol{\eta}_{2} x_{2,2}+\mathbf{G}_{12} \mathbf{H}_{11}^{-1} \boldsymbol{\eta}_{2} x_{1,2}+\boldsymbol{\eta}_{1}\left(x_{2,1}+x_{1,1}\right)+\mathbf{n} .
\end{aligned}
$$

As seen in (2.10), at receiver 1 , the undesired messages $x_{1,2}$ and $x_{2,2}$ are aligned 
along the dimension of $\boldsymbol{\eta}_{2}$. Similarly, it is given in (2.11) that the undesired messages of receiver $2, x_{1,1}$ and $x_{2,1}$ are aligned along the dimension of $\boldsymbol{\eta}_{1}$. As a result of alignment, the desired messages occupy linearly independent signal dimensions and can be isolated from undesired messages using cancellation methods in the receiver, such as $\mathrm{ZF}$ receivers.

Transmitter 1
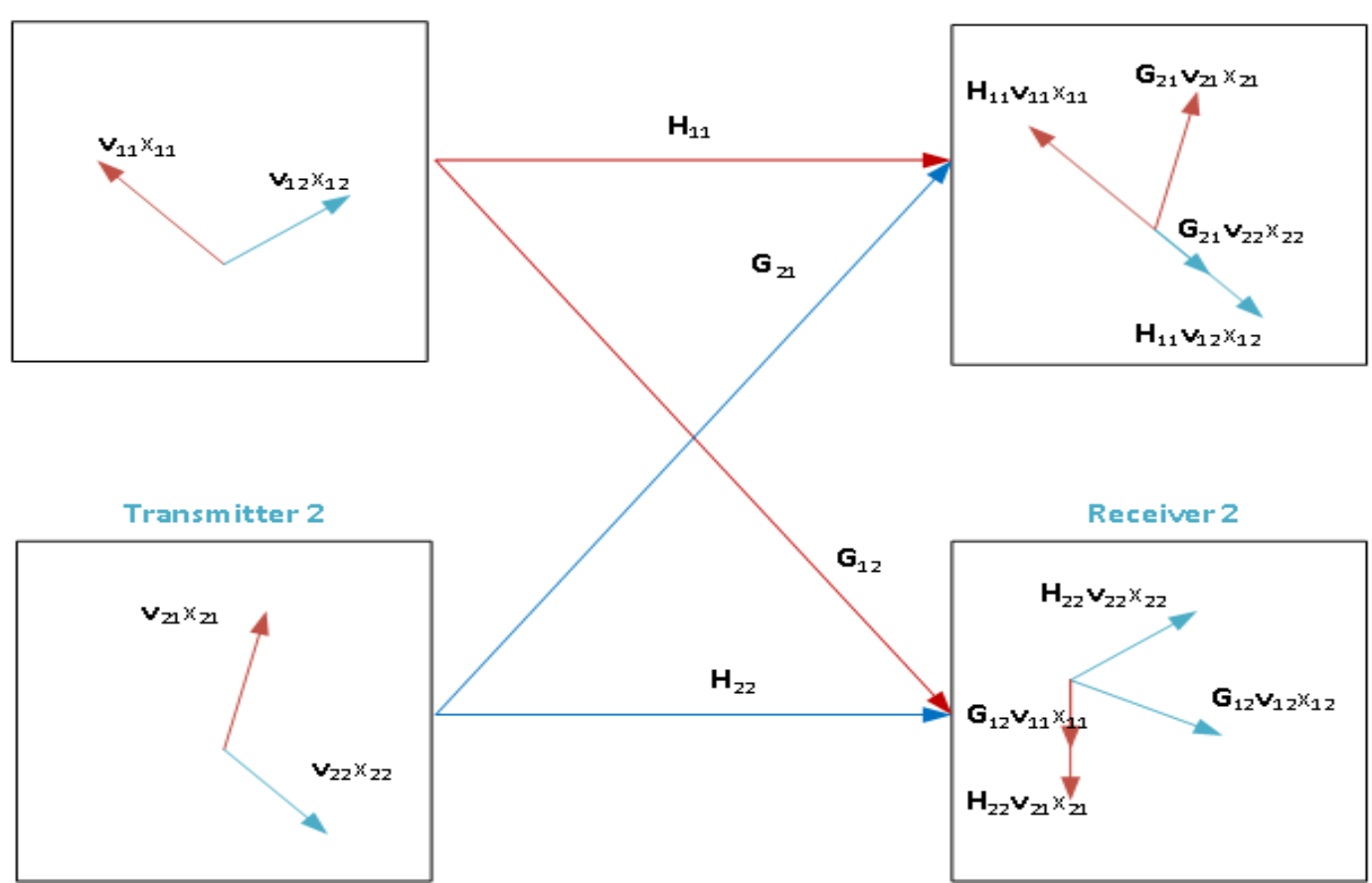

Figure 2.13: MIMO X channel model of [14].

The advantage of IA is that zero forcing one interference signal automatically zero forces both interfering signals as all interference signals aligned in the same subspace [9]. If we generate a receiver which is orthogonal to the interference subspace, we can cancel all interference at the same time.

\subsection{Intereference Alignment in Interference Chan- nels}

In this section, we discuss about IA in interference channels. The main difference between the $\mathrm{X}$ channel and the interference channel is the transmitted messages [9] [56].The $\mathrm{X}$ channel is the most general case where each transmitter has an independent message for each receiver, for a total of four independent messages as given in the previous section. The interference channel is a special case of $\mathrm{X}$ channel, where there is no message to be transmitted from transmitter 1 to receiver 2 , or from transmitter 2 to receiver 1 [9] This means the receiver 1 only 
desires to get messages from transmitter 1 , while the transmitter 2 only conveys messages for the receiver 2 .

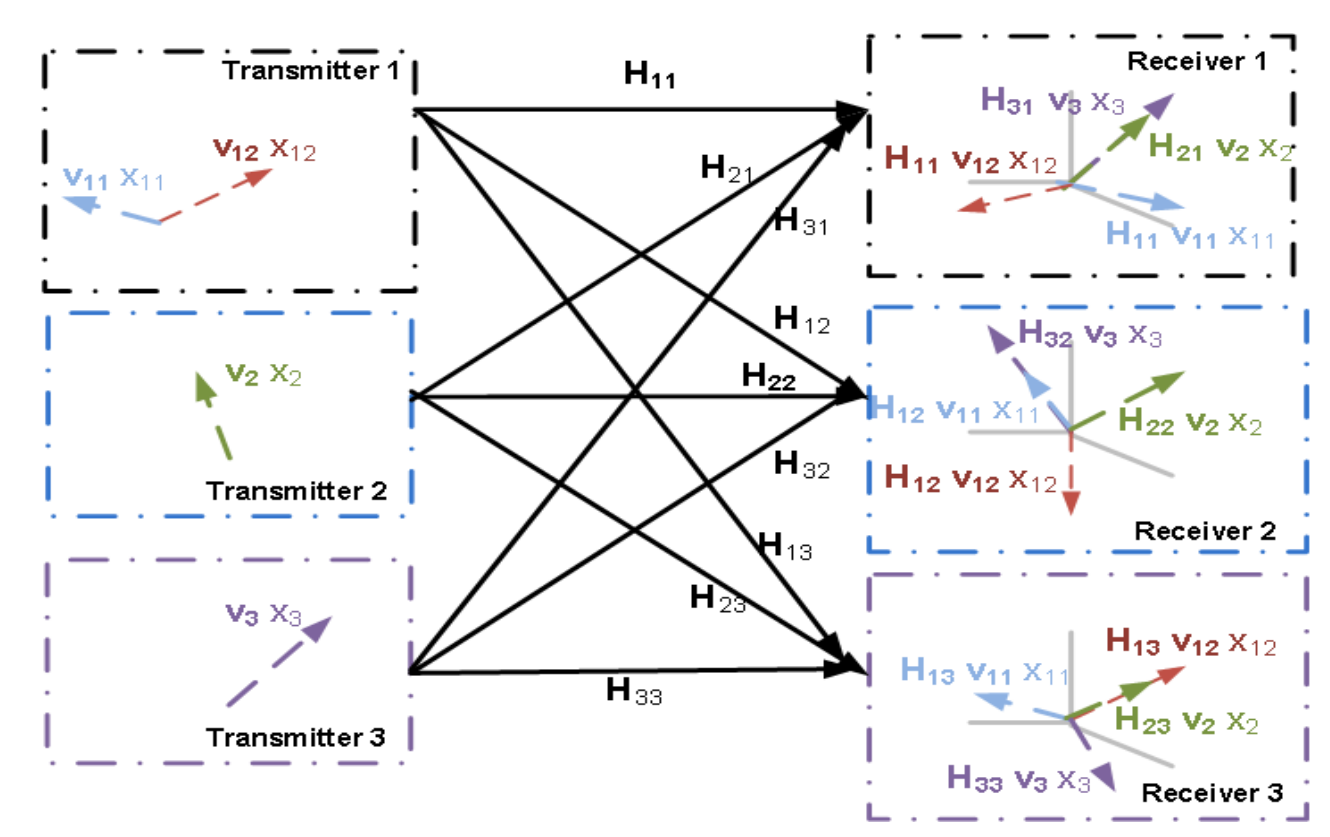

Figure 2.14: Interference alignment in an interference channel of [11]

\subsubsection{System Model}

Here, we give the IA scheme of [11] in an interference channel which consists of 3 users. User 1 transmits independently coded messages $x_{1,1}$ and $x_{1,2}$ along the precoding vectors $\mathbf{v}_{1,1}$ and $\mathbf{v}_{1,2}$, respectively. User 2 and user 3 , each transmit a single message as $x_{2}$ and $x_{3}$ along the precoding vectors $\mathbf{v}_{2}$ and $\mathbf{v}_{3}$, respectively. Such system is shown in Fig. 2.14. Let the $M$ symbol extension ${ }^{4}$ of the link from transmitter $k$ to receiver $j$ be denoted by a diagonal matrix $\mathbf{H}_{k j}(t)=$ $\operatorname{diag}\left\{h_{k j}(M t+1), \ldots, h_{k j}(M t+M)\right\}$ and $h_{k j}(\cdot)$ is the channel coefficient. As an example, we consider $M=3$. We first pick a reference precoding vector, such that

$$
\mathbf{v}_{2}=[1,1,1]^{T}
$$

Then we generate other precoders in order to align the interference in a subspace in each receiver. One could note here, there is no one unique way of generating precoders, $\mathbf{v}_{2}$ could be any random vector instead of all ones vector. We can generate other precoding vectors as follows

- At receiver 1 , the interference from transmitters 2 and 3 can be aligned

\footnotetext{
${ }^{4}$ Symbol extension of a channel consists of sending multiple copies of a symbol over space or time. Using a single antenna at the receiver and the transmitter, the symbol extension of a channel becomes a diagonal matrix.
} 
provided that

$$
\mathbf{H}_{21} \mathbf{v}_{2}=\mathbf{H}_{31} \mathbf{v}_{3}
$$

Therefore $\mathbf{v}_{3}$ could be given by

$$
\mathbf{v}_{3}=\left(\mathbf{H}_{31}\right)^{-1} \mathbf{H}_{21} \mathbf{v}_{2}=\left(\mathbf{H}_{31}\right)^{-1} \mathbf{H}_{21}[1,1,1]^{T} .
$$

- At receiver 2 , the interference from transmitter 3 can be aligned along one dimension of the interference signals from transmitter 1 such that

$$
\mathbf{H}_{32} \mathbf{v}_{3}=\mathbf{H}_{12} \mathbf{v}_{1,1} \text {. }
$$

So that one could write $\mathbf{v}_{1,1}$ as

$$
\begin{aligned}
\mathbf{v}_{1,1} & =\left(\mathbf{H}_{12}\right)^{-1} \mathbf{H}_{32}\left(\mathbf{H}_{31}\right)^{-1} \mathbf{H}_{21} \mathbf{v}_{2} \\
& =\left(\mathbf{H}_{12}\right)^{-1} \mathbf{H}_{32}\left(\mathbf{H}_{31}\right)^{-1} \mathbf{H}_{21}[1,1,1]^{T} .
\end{aligned}
$$

- At receiver 3, the interference from transmitter 2 can be aligned along one of the signal dimensions of interference from transmitter 1 such that

$$
\mathbf{H}_{23} \mathbf{v}_{2}=\mathbf{H}_{13} \mathbf{v}_{1,2}
$$

Thus, the precoding vector of $\mathbf{v}_{1,2}$ could be given by

$$
\begin{aligned}
\mathbf{v}_{1,2} & =\left(\mathbf{H}_{13}\right)^{-1} \mathbf{H}_{23} \mathbf{v}_{2} \\
& =\left(\mathbf{H}_{13}\right)^{-1} \mathbf{H}_{23}[1,1,1]^{T} .
\end{aligned}
$$

As mentioned earlier, the precoding vectors can be constructed in many ways, there is no one unique way. For example, at receiver 2, the interference from transmitter $3, \mathbf{H}_{32} \mathbf{v}_{3}$, does not necessarily have to be aligned with one of the dimensions of interference received from transmitter 1 . The necessary condition is that it needs to lie within the two-dimensional (2-D) space spanned by the two interference signals received from transmitter 1 such that [11]

$$
\mathbf{H}_{32} \mathbf{v}_{3} \in \operatorname{span}\left[\mathbf{H}_{12} \mathbf{v}_{1,1} \quad \mathbf{H}_{12} \mathbf{v}_{1,2}\right] .
$$

Similarly, at receiver 3 , the alignment can be done as follows

$$
\mathbf{H}_{23} \mathbf{v}_{2} \in \operatorname{span}\left[\mathbf{H}_{13} \mathbf{v}_{1,1} \quad \mathbf{H}_{13} \mathbf{v}_{1,2}\right],
$$

where $\operatorname{span}(\cdot)$ denotes the space spanned by the column vectors of a matrix. In the following chapter, we will discuss about a multi-hop interference channel 
which could be considered as the concatenation of 2 interference channels.

\subsection{Interference Alignment Challenges}

IA relies on some perfect assumptions which are not realistic for practical communication systems, that is why all these assumptions must be relaxed and worse cases must be considered before IA is adopted in today's wireless communication systems. In this section, we will explain these assumptions and the challenges that we face when we relax these assumptions.

\subsubsection{Dimensionality}

IA is achieved by some coding to align the interference signals to a smaller space. As a result of this, if more interference signals exist, we need more dimensions to align them. Prior work has shown that in frequency domain alignment, needed signalling dimensions grow faster than exponentially with the number of users [15]. This dimension challenge is relatively milder in the case of MIMO IA where more users can be supported as long as the number of antennas grow linearly with network size [15]. Considering the results of previous work, IA is more likely to be implemented in MIMO systems [15].

\subsubsection{Channel Estimation and Feedback}

CSI, either at transmitter or receiver, has a key importance to achieve IA with precoders. As a result of this, sufficient resources must be allocated to pilot transmission and to CSI feedback, to ensure accurate CSI [15]. Since IA precoders highly rely on the accurate knowledge of CSI, the channel changes must be applied as quickly as possible especially in high-mobility fading systems which can limit the performance of IA very drastically. We will later investigate erroneous CSI and limited feedback and their effect in some IA applications.

\section{Obtaining CSI in the Interference Channel}

Generating precoding vectors which are essential in aligning interference is very susceptible on CSI that is why transmitter must have accurate knowledge of geometry of the interference it creates, then it can conceivably align the interference that it creates. There are two methods of obtaining CSI knowledge at transmitter: reciprocity and feedback [15].

\section{Reciprocity}

The main advantage of TDD systems compared to FDD systems is the possibility to exploit channel reciprocity [15]. If channel reciprocity holds, both 
forward and reverse transmission links match exactly. Then, the channel estimate of the uplink direction at the transmitter can directly be utilised for link adaptation in the downlink. Reciprocity enables IA by allowing transmitters to infer the structure of the interference they cause by observing the interference they receive. Regardless of the IA scheme, precoding with reciprocity can be implemented as follows [15]

- Forward Link Training: Transmitters send precoded pilot symbols after initializing a set of precoders. Receivers estimate forward channel parameters and compute postcoding vectors.

- Reverse Link Training: Receivers send precoded pilot symbols using the postcoding vectors from step 1 as transmit precoders. Transmitters in return optimise their postcoders/ precoders and initiate a second training phase with the updated precoders.

- Communicating pairs iterate the previous steps until convergence.

- Data transmission: The desired data is then communicated.

On the other hand, precoding with reciprocity has such a drawback that iterating over the air incurs a non-negligible overhead due to the recurring pilot transmissions [57]. Reciprocity also can not be used when uncoordinated interference observed by transmitters and receivers are not reciprocal [15].

\section{Feedback}

Several IA schemes in the literature have considered CSI feedback [15]. In such systems, the transmitters first send training sequences then receivers estimate the forward channels using these training sequences. Later, receivers feed back information of the estimated forward channels. After feedback, the transmitters have the channel information that is needed to generate IA precoding vectors. The main challenge encountered in IA systems with CSI feedback is designing low-overhead low-distortion feedback schemes.

One of the most common used method to provide high quality feedback with low overhead is limited feedback, i.e., channel state quantization [58]. However, in order to align interference properly, the accuracy of quantised CSI is dependent on the quantised codebook size. The complexity of quantised feedback increases with codebook size and large codebooks are difficult to design and encode. Another drawback that we face with CSI quantization is the fact that it can not be applied to systems where the CSI exhibits no special structure [15]. 


\subsubsection{Interference Alignment in Large Scale Networks}

Applying IA schemes into large scale networks such as cellular networks is very challenging as a result of the growing need of more signalling dimensions, the exploding overhead of CSI acquisition and also synchronization [15]. Despite its complexity, it can be simplified as not all interfering links are significant and that is why not all of these interfering links should be treated equally. Therefore, dominant and remaining interference that we discussed in previous sections should be handled in different ways, which will be discussed in detail in Chapter 4.

\subsubsection{Synchronization}

As a transmission strategy for the coherent interference channel, IA requires perfect synchronization to remove any timing and frequency offsets between the cooperating nodes. If there is no precise synchronization, additional interference terms will be introduced which will result in inefficient IA. In [15], it is mentioned that synchronization strategies used in satellite systems could fulfil this requirement.

\subsection{Imperfect Channel State Information and Lim- ited Feedback}

\subsubsection{Modelling erroneous CSI}

In this thesis, we model the CSI and CCSI imperfections as in [59]. Therefore

$$
\begin{aligned}
\hat{\mathbf{H}} & =\varsigma \mathbf{H}+\bar{\zeta} \boldsymbol{\Pi} \\
\hat{\mathbf{G}} & =\varsigma \mathbf{G}+\bar{\varsigma} \boldsymbol{\Pi}
\end{aligned}
$$

where $\varsigma, 0<\varsigma<1$ and $\bar{\varsigma}=\sqrt{1-\varsigma^{2}}$, control the amount of CSI imperfection (i.e., $\varsigma=1$ refers to perfect $\mathrm{CSI}$ ). $\mathbf{H}, \mathbf{G}$ are the channel matrices. Depending on the channel matrix, $\Pi$ can be in different forms. For example if $\mathbf{H}, \mathbf{G}$ are SISO channels with symbol extension as mentioned in Chapter 3 - Section 3.1, $\Pi$ is a diagonal complex Gaussian matrix with zero mean and unit variance. If they are MIMO channels without symbol extension, $\Pi$ is a $\left(N_{r} \times N_{t}\right)$ complex Gaussian matrix. It is shown in [60] and [61] that $\varsigma$ can be used to determine the impact of several factors on imperfect CSI and can be a function of the length of the training sequence, SNR and Doppler frequency. 


\subsubsection{Limited Feedback}

There has been extensive research in the literature to overcome the challenge of making instantaneous channel adaptation practical. One of the approaches is the use of feedback. The feedback information itself can be digital or analog. In this section, we focus on digital feedback, which is commonly referred to as limited feedback or finite-rate feedback [62].

Detailed analysis of codebook design is given in [63]. The effect of estimation error and feedback delay is discussed in [64]. Grassmannian and vector quantization (VQ) limited feedback are fixed designs and the codebooks do not vary as the channel changes. Another approach is to randomly generate the codebook at each block. These randomly generated codebooks are perfectly known to both the transmitter and receiver. This sort of codebook design technique is known as random vector quantization (RVQ) and was first proposed in $[65,66]$. The quantization of codebooks can be classified into two categories: Structured and unstructured [67]. The structured codebooks [68-71] parametrise the codewords with some matrix operations (i.e. multiplication, Givens rotation [72], etc.), and as a result of this, its storage complexity is significantly low. The unstructured [73] codebooks, however, optimise each codeword over a distribution of the beamforming matrix. As mentioned in [67], common optimization metrics for the unstructured codebooks are chordal distance [74] and mutual information [69]. Unstructured codebooks outperform structured ones, but the unstructured codebooks also require higher cost of storage requirements [67].

\section{IEEE 802.16e codebooks}

IEEE $802.16 \mathrm{e}$ is the mobile extension to IEEE 802.16, for wireless networks in metropolitan areas [75]. It is also known as WiMAX (Wireless Interoperability for Microwave Access). IEEE 802.16e has various physical layers and MIMO modes of operation. Several single-user codebook based limited feedback techniques are supported in IEEE 802.16e [67]. The scheme adopted by $802.16 \mathrm{e}$ is a combination of both the structured and unstructured approaches to meet the storage, multi - rate, and multi-configuration requirements [67]. The small codebooks for MISO channels (i.e. $2 \times 1$ ) are unstructured and stored, while large codebooks for MIMO channels (i.e. $4 \times 2$ ) are structured that can be efficiently computed from the stored codebooks using Householder reflection [72]. In order to provide further storage reduction, the structured codebooks is derived for MISO channels (i.e. $3 \times 1$ and $4 \times 1$ ) with 64 codewords. These codebooks are modified from [68]. The feedback and processing delay of these codebooks is suppressed with a channel prediction algorithm based on Wiener filtering [67]. 


\subsection{Antenna Correlation}

A degradation on the capacity with multiple antenna techniques can occur due to the correlation between individual channels of the matrix channel. Increase in the correlation coefficient results in capacity decrease and, finally, when the correlation coefficient equals to unity, no advantage is provided by the multiple antenna architecture. In this thesis, we use a correlation model given in [76] as;

$$
\mathbf{H}=\mathbf{L}^{\frac{1}{2}} \mathbf{H}^{\prime},
$$

where $\mathbf{H}^{\prime}$ is the random complex Gaussian channel matrix. $\mathbf{L}$ is the correlation matrix of which elements are

$$
\mathbf{L}_{i j}=\left\{\begin{array}{ll}
\xi^{j-i} & \text { if } i \leq j \\
\mathbf{L}_{i j}^{*} & \text { if } i>j .
\end{array},\right.
$$

where $i, j=1,2, \ldots M, \mathrm{M}$ is the antenna number, $\xi$ is a complex value and $0 \leq|\xi| \leq 1$. The cases of $|\xi|=0$ and $|\xi|=0.9$ indicate spatially independent and strongly correlated channels respectively.

\subsection{Literature Review}

Here, we will review some key papers of IA under three main sections,

\subsubsection{IA Applications}

IA was proposed in [11] and [12] for the first time. Cadambe and Jafar showed in [11] that interference-free communication could be provided to $K$ users for a $1 / 2$ of the time which means, regardless of the number of users, everyone gets the half of the cake. This was a significant improvement and took interest of many researchers which led a vast literature on IA. In [11], the IA scheme was also extended to $K$ user MIMO Interference Networks where the transmitter and the receivers have the same number of antennas. It was noted that CSI has to be known at both the transmitters and the receivers for perfect alignment. Later, the authors of [77] studied the case of unequal number of antennas at all transmitters and receivers. They provided an analytical insight on the performance of the IA scheme. They also provided examples where using IA combined with ZF can achieve better performance than merely ZF for some MIMO interference channels with constant channel coefficients. Again the channels have to be fully connected and CSI has to be perfectly known by transmitters and receivers. 
The authors of [78] compared two distributed IA techniques : IA in space and IA in frequency. Both schemes aimed removing interference through orthogonalization. It was shown that frequency IA requires less complexity to achieve higher multiplexing gain and spectral efficiency than space IA. However, frequency alignment is less efficient in terms of INR (results in higher interference) and the spectral efficiency per link thus decreases when the number of links increases.

In [79], the authors studied a MIMO Gaussian wireless network with three users, where each user intends to convey independent messages for two different users via the relay while receiving two independent messages from the other two users. This is a generalised version of the two-way relay channel for a three user case. Gou et al of [2] studied a SISO multihop network consisting of two sources, two relays and two destinations, where the first hop is between the sources (transmitters) and the relays and the second hop is between the relays and the destinations (receivers). This setting can be considered as a concatenation of 2 interference channels. In this paper, the authors proposed aligned interference neutralisation as a way to align the interference over each hop of the network leading the interference to be cancelled out over the last hop. This achieves the 2 degrees of freedom. However, the remarkable benefit of aligned interference neutralisation was shown under idealised assumptions, i.e. the availability of perfect channel knowledge which is not realistic in wireless networks. We will provide an analytical analysis of this technique in detail, considering imperfect CSI in Chapter 3.

\subsubsection{Cellular Networks in Uplink}

We have shown in Section 2.3.1 that the uplink system suffers from interference significantly. IA for uplink systems were considered in [80-85]. In [80], a two cell interfering two-user MIMO MAC with limited feedback was considered. First, the MIMO Interference scheme was implemented with perfect feedback. In addition to this, the authors applied a feedback framework given in [81], and then proposed a new feedback scheme for IA. On the basis of the proposed feedback framework, the rate gap loss was analysed and it was shown that in order to keep the same multiplexing gain with the case of perfect CSI at transmitters, the number of feedback bits per receiver should be scaled with respect to the received SNR.

In [82], a new limited feedback scheme for IA in Two-Cell Interfering MIMOMAC was proposed. According to this scheme, the receiver determines its beamforming vectors using quantised transmitter vectors. This scheme provided slightly better performance than the scheme given in [80]. They analysed that the rate loss was only impacted by the residual intercell interference. By 
characterizing the rate loss as a function of the number of feedback bits, a bits allocation algorithm can be introduced as given in [80].

In [85], the authors proposed to jointly optimise transmitter and receiver sets for MIMO uplink systems. They optimised the system minimizing the total MSE. They showed that minimizing the MSE function is a jointly convex optimization problem. Later in Chapter 5, we will follow a similar way to optimise a multicell network in the uplink and improve the system performance significantly.

\subsubsection{Cellular Networks in Downlink}

The downlink also suffers from interference heavily as given in Section 2.3.2. Therefore IA is very promising for the downlink. IA schemes for downlink systems were considered in [3,86-90]. Authors of [86] studied a K-cell MISO interfering broadcast channel with limited feedback, where the BSs equipped with multiple antennas wish to communicate with users with a single antenna based on the quantised CSI. The main contributions are that coordinated ZF beamforming with limited CSI in K-cell MISO is proposed, rate loss per user due to limited feedback is characterised and a closed form feedback bits allocation scheme which minimizes the expected quantization error is proposed.

The scheme proposed in [87] is very similar to [80], written by the same authors. In [87], they focused on IA for interfering downlink channel, instead of uplink. But the IA scheme that they used is almost the same as [80]. The algorithm required only a small amount of channel feedback information with the aid of the user cooperation channels. The simulations demonstrated that not only were the analytical results valid, but the proposed algorithm also outperformed those of conventional techniques to deal with interference.

IA on cellular networks with imperfect CSI was on the focus of [88]. However in their system they considered $\mathrm{K}$ cells with 1 user equipped with multiple antennas in each cell which is same as the $\mathrm{K}$ user MIMO interference channel. Main contribution was that upper/lower bounds were derived for the case of imperfect CSI. Opportunistic IA was proposed in [89] for the secondary users exploiting the fact that under a power-limitation, a primary user that maximizes its own rate by water-filling on its MIMO channel singular values, leaving some of them unused. These unused directions may be opportunistically utilised by a secondary transmitter since its signal would not interfere with the signal sent by the primary transmitter.

Main contribution of [90] was the subspace IA for more - than - two - cell cases, which was to align interference into multi-dimensional subspace for simultaneous alignments at multiple non-intended BSs. The proposed scheme required finite dimensions growing linearly with user number in each cell.

In [3], authors developed an IA technique for a downlink cellular network. In 
the previous IA uplink schemes, CSI exchange across BSs of different cells was required, but in this downlink scheme IA required feedback only within a cell. The scheme provided a substantial gain especially when interference from a dominant interferer was significantly stronger than the remaining interference. It was also mentioned in this paper that the proposed scheme had the potential to provide substantial gain for macro-pico cellular networks where pico-users could be significantly interfered with by the nearby macro-BS. They observed that zero-forcing IA scheme is analogous to the $\mathrm{ZF}$ receiver, and the iterative matched-filtering (MF) technique corresponds to the MF receiver. Based on this, the authors proposed a unified IA scheme similar to an MMSE receiver that outperforms both techniques where the power of the dominant interferer may be much greater or smaller than the power of the remaining aggregate interference. We will explain the scheme of [3] in detail in Chapter 4.

Precoding vectors have been extensively used to align and cancel interference. The idea of alignment is to design transmitted vectors so that they are aligned onto a linear subspace at the receiver [3]. ZF methods for MIMO interference channels were considered in [91]. ZF precoders require a condition on the number of transmit-receive antennas. According to this condition, the number of transmit antennas needs to be equal or larger than the total number of receive antennas of the users to be nulled. If this condition is not satisfied, then the interference cannot be nulled. Signal-to-leakage-noise-ratio (SLNR) based precoders were proposed in [92] so that the interference can be cancelled without any antenna requirement, contrary to ZF precoders. These precoders will be further explained in Chapter 5.

Spectral efficiency of cell edge is very important in the design of cellular networks. In order to improve cell-edge spectral efficiency, the authors of [93-95] considered cooperation between the BSs. Currently Coordinated Multi-Point transmission/reception (CoMP) systems allow coordination among 3 adjacent cells [96]. The coordination can be implemented in the overlapping regions where the coverage area of adjacent cells overlap. The authors of [97] showed that the overlapping region can cover $30-47 \%$ of the total area of two neighbouring cells. In [97], the overlapping region was assumed to be where the difference of the received power from 2 adjacent BSs is within $8 \mathrm{~dB}$. In Chapter 5 , we will consider an IA scheme with cooperation in a multicellular network.

\subsection{Summary}

In this chapter we have presented the technical background and preliminary work which this thesis has been motivated by. First, an introduction to the wireless channels has been given, followed by a definition of MIMO systems. Later, cellular networks have been discussed and some simulation results for 
both uplink and downlink systems have been provided to demonstrate how much cellular networks suffer from interference. The imperfect channel model and the correlation model that we will use in the following chapters have also been given in this chapter. We have also discussed traditional methods to deal with interference, mentioning their drawbacks. We have given some simple examples of IA such as for MIMO X channels and interference channels. In the following section, the challenges of IA systems were discussed. Finally we have concluded this chapter with a literature review on IA applications as well as cellular networks in uplink and downlink. 


\section{3}

\section{Interference Alignment in Multihop Relay Networks}

In this chapter, an IA scheme is given for a multihop relay network, which also provides an introduction to more complex cellular networks which will be considered in the following chapters. At the beginning, we review a 2 user - 2 transmitter - 2 receiver system. We derive analytical expressions for the SNR and SINR at relays and receivers. We also consider the effects of imperfect CSI and show that the interference caused by misalignment due to CSI errors is very prominent.

\subsection{Interference Alignment in Multihop Interfer- ence Channels}

In this section, we present an IA application in a multihop network where relays are employed. Authors of $[2,98]$ studied a SISO network consisting of two sources, two relays and two destinations, where the first hop is between the sources (transmitters) and the relays and the second hop is between the relays and the destinations (receivers). This setting can be considered as a concatenation of 2 interference channels. Jafar and Cadambe showed in [98] that this muti-hop relay network which consists of 2 transmitters, 2 relays and 2 receivers, can achieve $\frac{4}{3}$ degrees of freedom. This result was achieved by a decode and forward approach which treats each hop as an X channel which was explain in Chapter 2, Section 2.6. Authors of [2] proposed aligned interference neutralisation as a way to align the interference over each hop of the network leading the interference to be cancelled out over the last hop. This technique 


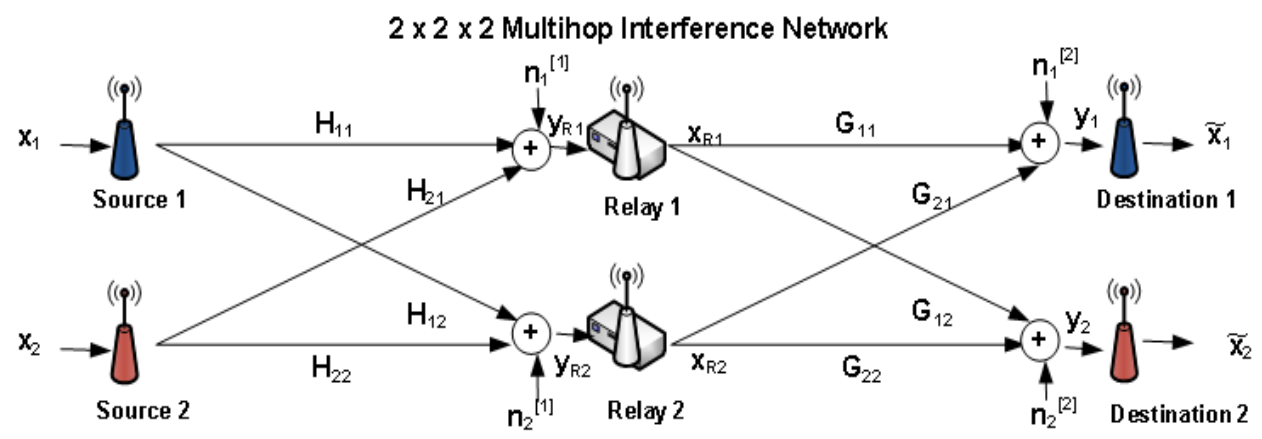

Figure 3.1: 2 user - 2 transmitter - 2 relay - 2 receiver $(2 \times 2 \times 2)$ Network of [2]

of [2] achieves the 2 degrees of freedom which is higher that what was proposed in [98] before. However, the remarkable benefit of aligned interference neutralisation has been shown under idealised assumptions i.e. the availability perfect channel knowledge which is not realistic in wireless networks. In this section, we present the results that we published in [99] where we analysed the effects of imperfect channel state information (CSI) on the performance of aligned interference neutralisation for a 2 transmitter - 2 relay - 2 receiver channel of [2] as shown in Fig. 3.1. This is referred to as a $2 \times 2 \times 2$ network. Channel estimation errors cause erroneous precoding vectors and thus misalignment of desired and interference signals. Our contributions are as follows [99]

- We derive analytical expressions for the SNR and SINR at the relays. For the former we show that, due to the ZF at the relays, the expected value of the noise tends to infinity. Similarly the interference caused by misalignment due to CSI errors is shown to be very prominent with the mean tending to infinity.

- We demonstrate that the SINR analysis for the relays can be extended to the destinations and show that there are cumulative effects of interference and noise at both stages.

- We validate the analysis through system simulations. We show that the interference due to CSI errors can be modelled via simulation by a log normal distribution, thus confirming the presence of prominent instantaneous values of interference.

\subsubsection{System Model}

\section{Time varying Channel Coefficients and Perfect CSI}

We consider the time varying channel coefficient case in [2] where the transmission occurs over $M$ time slots. Transmitters 1 and 2 encode $M$ and $M-1$ 


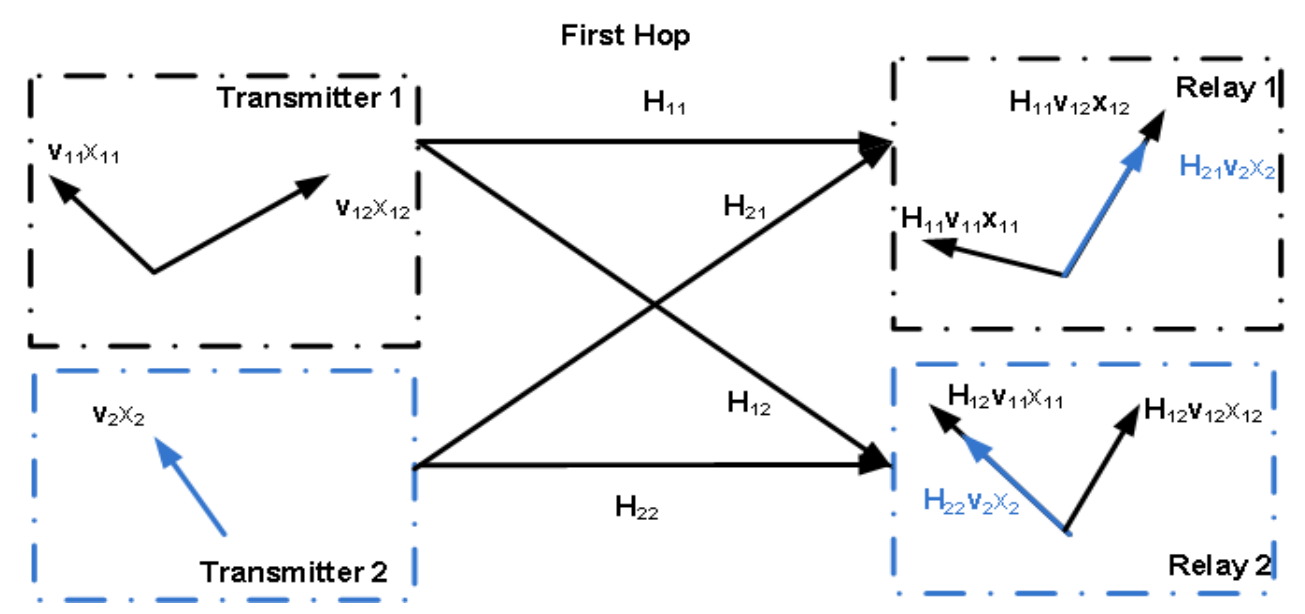

Figure 3.2: First hop of the network of [2]

data symbols $x_{1, k_{1}}, x_{2, k_{2}}$ respectively, using precoding vectors $\mathbf{v}_{1, k_{1}}$ and $\mathbf{v}_{2, k_{2}}$, resulting in $(M \times 1)$ data vectors

$$
\begin{aligned}
& \mathbf{x}_{1}=\sum_{k_{1}=1}^{M} \mathbf{v}_{1, k_{1}} x_{1, k_{1}} \\
& \mathbf{x}_{2}=\sum_{k_{2}=1}^{M-1} \mathbf{v}_{2, k_{2}} x_{2, k_{2}} .
\end{aligned}
$$

In the case of perfect CSI, the precoding vectors are designed to align the interference at the relays as given in Fig. 3.2 [2] and are given by

$$
\begin{aligned}
\mathbf{v}_{1, i+1} & =\left(\mathbf{H}_{11}^{-1} \mathbf{H}_{21} \mathbf{H}_{22}^{-1} \mathbf{H}_{12}\right)^{i} \mathbf{v}_{1,1} \\
\mathbf{v}_{2, i} & =\left(\mathbf{H}_{22}^{-1} \mathbf{H}_{12} \mathbf{H}_{11}^{-1} \mathbf{H}_{21}\right)^{i-1} \mathbf{H}_{22}^{-1} \mathbf{H}_{12} \mathbf{v}_{1,1}
\end{aligned}
$$

where $\mathbf{v}_{1,1}$ is arbitrarily set to $\mathbf{v}_{1,1}=[1,1, \ldots, 1]^{T}, \quad \forall i \in\{1, \cdots, M-1\}$. Let the $M$ symbol extension of the link from Transmitter $k$ to relay $j$ be denoted by a diagonal matrix $\mathbf{H}_{k j}(t)=\operatorname{diag}\left\{h_{k j}(M t+1), \ldots, h_{k j}(M t+M)\right\}$. Similarly the links between relay $j$ and receiver $n$ are given by $\mathbf{G}_{j n}(t)=\operatorname{diag}\left\{g_{j n}(M t+\right.$ $\left.1), \ldots, g_{j n}(M t+M)\right\}$. Then the $(M \times 1)$ signal vectors received at relay $R_{j}$ and the receiver $n$ where $j=1,2$ and $n=1,2$, are

$$
\begin{aligned}
\mathbf{y}_{R_{j}}(t) & =\mathbf{H}_{1 j}(t) \mathbf{x}_{1}(t)+\mathbf{H}_{2 j}(t) \mathbf{x}_{2}(t)+\mathbf{n}_{j}^{[1]}(t) \\
\mathbf{y}_{n}(t) & =\mathbf{G}_{1 n}(t) \mathbf{x}_{R_{1}}(t)+\mathbf{G}_{2 n}(t) \mathbf{x}_{R_{2}}(t)+\mathbf{n}_{n}^{[2]}(t),
\end{aligned}
$$

where, $\mathbf{n}_{j}^{[1]}(t)$ and $\mathbf{n}_{n}^{[2]}(t)$ denote the noise vectors with superscripts [1] and [2] referring to the first and second hops, respectively. Omitting the time dependence of channel coefficients for notation simplicity, the alignment conditions 
result in

$$
\begin{aligned}
\mathbf{y}_{R_{1}} & =\mathbf{H}_{11} \mathbf{x}_{1}+\mathbf{H}_{21} \mathbf{x}_{2}+\mathbf{n}_{1}^{[1]} \\
& =\mathbf{H}_{11} \mathbf{v}_{1,1} x_{1,1}+\sum_{i=1}^{M-1} \mathbf{H}_{11} \mathbf{v}_{1, i+1}\left(x_{1, i+1}+x_{2, i}\right)+\mathbf{n}_{1}^{[1]},
\end{aligned}
$$

and similarly

$$
\begin{aligned}
\mathbf{y}_{R_{2}} & =\mathbf{H}_{12} \mathbf{x}_{1}+\mathbf{H}_{22} \mathbf{x}_{2}+\mathbf{n}_{2}^{[1]} \\
& =\sum_{i=1}^{M-1} \mathbf{H}_{12} \mathbf{v}_{1, i}\left(x_{1, i}+x_{2, i}\right)+\mathbf{H}_{12} \mathbf{v}_{1, M} x_{1, M}+\mathbf{n}_{2}^{[1]}
\end{aligned}
$$

Interference is neutralised by applying $\mathrm{ZF}$ at the relays, giving the $(M \times 1)$ processed received vectors

$$
\left[\begin{array}{c}
x_{R_{1}, 1} \\
x_{R_{1}, 2} \\
\vdots \\
x_{R_{1}, M}
\end{array}\right]=\mathbf{H}_{R_{1}}^{-1} \mathbf{y}_{R_{1}}=\left[\begin{array}{c}
x_{1,1} \\
x_{1,2}+x_{2,1} \\
\vdots \\
x_{1, M}+x_{2, M-1}
\end{array}\right]+\mathbf{H}_{R_{1}}^{-1} \mathbf{n}_{1}^{[1]}
$$

and

$$
\left[\begin{array}{c}
x_{R_{2}, 1} \\
x_{R_{2}, 2} \\
\vdots \\
x_{R_{2}, M-1} \\
x_{R_{2}, M}
\end{array}\right]=\mathbf{H}_{R_{2}}^{-1} \mathbf{y}_{R_{2}}=\left[\begin{array}{c}
x_{1,1}+x_{2,1} \\
x_{1,2}+x_{2,2} \\
\vdots \\
x_{1, M-1}+x_{2, M-1} \\
x_{1, M}
\end{array}\right]+\mathbf{H}_{R_{2}}^{-1} \mathbf{n}_{2}^{[1]},
$$

where $\mathbf{H}_{R_{j}}=\left[\mathbf{H}_{1 j} \mathbf{v}_{1,1} \mathbf{H}_{1 j} \mathbf{v}_{1,2} \cdots \mathbf{H}_{1 j} \mathbf{v}_{1, M}\right]$ is the $(M \times M)$ ZF matrix at relay $R_{j}$.

The relays transmit the signals as in Fig. 3.3, to the destinations along precoding vectors $\mathbf{v}_{R_{1}, i+1}$ and $\mathbf{v}_{R_{2}, i}$ defined

$$
\begin{aligned}
\mathbf{v}_{R_{1}, i+1} & =\left(\mathbf{G}_{11}^{-1} \mathbf{G}_{21} \mathbf{G}_{22}^{-1} \mathbf{G}_{12}\right)^{i} \mathbf{v}_{R_{1}, 1} \\
\mathbf{v}_{R_{2}, i} & =-\left(\mathbf{G}_{22}^{-1} \mathbf{G}_{12} \mathbf{G}_{11}^{-1} \mathbf{G}_{21}\right)^{i-1} \mathbf{G}_{22}^{-1} \mathbf{G}_{12} \mathbf{v}_{R_{1}, 1},
\end{aligned}
$$

where once again $\mathbf{v}_{R_{1}, 1}=[1,1, \ldots, 1]^{T}$.

Following the precoding vectors at the relays using (3.13) and (3.14), the re- 


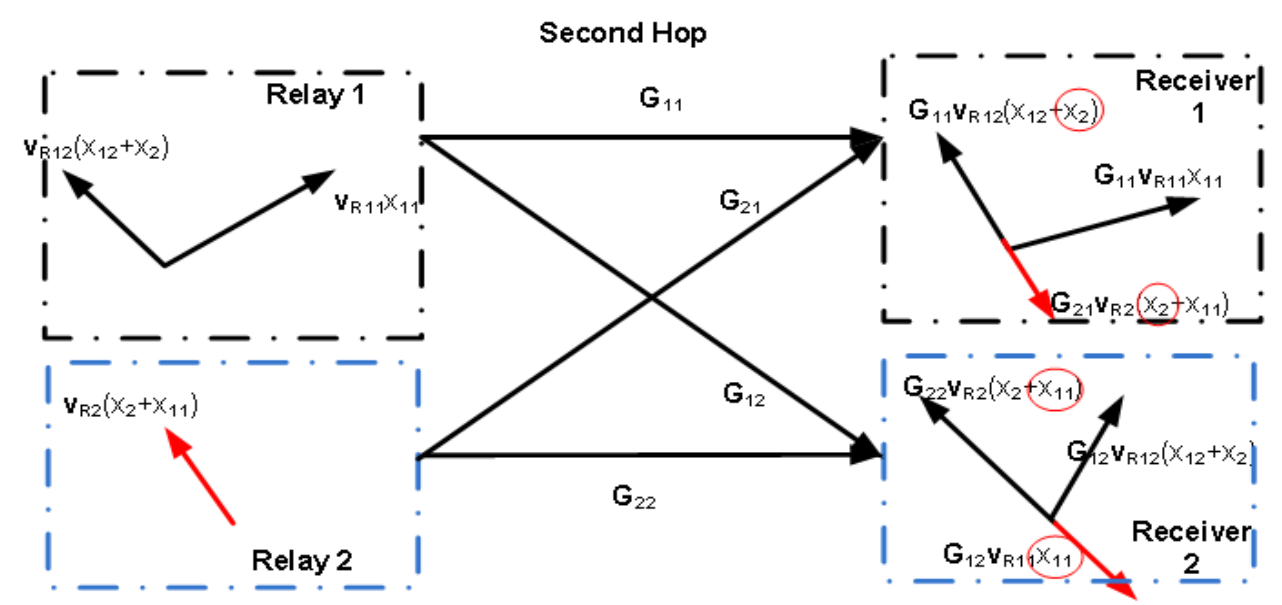

Figure 3.3: Second hop of the network of [2]

ceived signals at destinations 1 and 2 can be shown to be given by

$$
\begin{aligned}
\mathbf{y}_{1} & =\mathbf{G}_{11} \mathbf{X}_{R_{1}}+\mathbf{G}_{21} \mathbf{X}_{R_{2}}+\mathbf{n}_{1}^{[2]} \\
& =\mathbf{G}_{11} \mathbf{v}_{R_{1}, 1}\left(x_{1,1}+\tilde{n}_{1,1}\right) \\
& +\sum_{i=1}^{M-1} \mathbf{G}_{11} \mathbf{v}_{R_{1}, i+1}\left(x_{1, i+1}-x_{1, i}+\tilde{n}_{1, i+1}-\tilde{n}_{2, i}\right)+\mathbf{n}_{1}^{[2]},
\end{aligned}
$$

and

$$
\begin{aligned}
\mathbf{y}_{2} & =\mathbf{G}_{12} \mathbf{X}_{R_{1}}+\mathbf{G}_{22} \mathbf{X}_{R_{2}}+\mathbf{n}_{2}^{[2]} \\
& =\mathbf{G}_{12} \mathbf{v}_{R_{1}, M}\left(x_{1, M}+x_{2, M-1}+\tilde{n}_{1, M}\right) \\
& +\sum_{i=1}^{M-1} \mathbf{G}_{22} \mathbf{v}_{R_{2}, i}\left(x_{2, i}-x_{2, i-1}-\tilde{n}_{1, i}+\tilde{n}_{2, i}\right)+\mathbf{n}_{2}^{[2]},
\end{aligned}
$$

where $\tilde{n}_{1, k_{1}}$ and $\tilde{n}_{2, k_{2}}$ are the $k_{1}$ th and $k_{2}$ th element of $\mathbf{H}_{R_{1}}^{-1} \mathbf{n}_{1}^{[1]}$ and $\mathbf{H}_{R_{2}}^{-1} \mathbf{n}_{2}^{[1]}$ in (3.11) and (3.12) respectively. Finally each destination decodes the data symbols successively along each dimension as described in [2]. After neutralization, the receiver 1 has $x_{1,2}-x_{1,1}$ along the direction of $\mathbf{G}_{11} \mathbf{v}_{R_{1}, 2}$ and $x_{1,1}$ along the direction of $\mathbf{G}_{11} \mathbf{v}_{R_{1}, 1}$. Therefore it can first decode $x_{1,1}$ and then $x_{1,2}$ successively. The receiver 2 receives $x_{2}$ interference free along the dimension of $\mathbf{G}_{22} \mathbf{v}_{R_{2}}$ with discarding the dimension of $\mathbf{G}_{12} \mathbf{v}_{R_{1}, 2}$.

As mentioned in [2], over $M$ symbol extensions of the original channels, transmitter 1 can achieve $M$ degrees of freedom while transmitter can achieve $M-1$ degrees of freedom, for a total of $2 M-1$ degrees of freedom. Thus, the system can achieve $\frac{2 M-1}{M}$ degrees of freedom. As $M \rightarrow \infty$, almost surely, 2 degrees of freedom can be achieved [2]. 


\subsubsection{Performance Analysis}

We now derive analytical expressions of SNR for perfect CSI and SINR for imperfect CSI model in (2.23) for the first hop. We then extend this to end-toend SINR for imperfect CSI. For mathematical clarity, we consider the case of $M=2$. Expressions for $M>2$ are not considered but can be derived following similar methodology.

\section{SNR for Perfect CSI}

Consider the received vector at Relay 1 in (3.11), which for $M=2$, we denote by $\mathbf{x}_{R_{1}}=\left[\begin{array}{ll}x_{R_{1}, 1} & x_{R_{1}, 2}\end{array}\right]^{T}$. Using (3.11), after straightforward manipulation, one can show that

$$
\begin{aligned}
\mathbb{E}\left[\mathbf{x}_{R_{1}} \mathbf{x}_{R_{1}}^{*}\right] & =\left[\begin{array}{cc}
\mathbb{E}\left|x_{1,1}\right|^{2} & 0 \\
0 & \mathbb{E}\left|x_{1,2}\right|^{2}+\mathbb{E}\left|x_{2,1}\right|^{2}
\end{array}\right] \\
& +\sigma^{2} \mathbb{E}\left[\mathbf{H}_{R_{1}}^{-1} \mathbf{H}_{R_{1}}^{-1 *}\right]
\end{aligned}
$$

where $\sigma^{2}$ is the noise power. Using the definition of $\mathbf{H}_{R_{j}}$ given in Section 3.1.1 (after (3.12)), gives the SNR for symbol $x_{1,1}$

$$
\Gamma_{\text {inst }_{x_{1,1}}}=\frac{\mathbb{E}\left|x_{1,1}\right|^{2}}{\frac{\sigma^{2}}{|\Delta|^{2}} \Delta_{1}},
$$

where $\Delta_{1}$ and $\Delta$ given in terms of the instantenous channel coefficients are

$$
\Delta_{1}=\left|h_{21}(2)\right|^{2}\left|h_{12}(2)\right|^{2}\left|h_{22}(1)\right|^{2}+\left|h_{21}(1)\right|^{2}\left|h_{12}(1)\right|^{2}\left|h_{22}(2)\right|^{2},
$$

and

$$
\Delta=h_{11}(1) h_{22}(1) h_{21}(2) h_{12}(2)-h_{21}(1) h_{12}(1) h_{11}(2) h_{22}(2),
$$

with $h_{k j}(l)$ denoting the $l^{\text {th }}$ diagonal entry of $\mathbf{H}_{k j}$ For detailed explanation of (3.20), please see Appendix A.

To investigate the properties of (3.20), we examine the expected value of $\frac{\Delta_{1}}{|\Delta|^{2}}$ denoted by

$$
T=\mathbb{E}\left[\frac{\Delta_{1}}{|\Delta|^{2}}\right] .
$$

Considering (3.21) and (3.22), the nominator and the denominator of (3.23) has some common terms, except $h_{11}(1)$ and $h_{11}(2)$ in the denominator. Our aim is to simplify (3.23). Taking the expectation first over $h_{11}(1)$ and $h_{11}(2)$ while fixing the others, and then taking expactation over the rest of the variables, we obtain 


$$
T=\mathbb{E}\left[\mathbb{E}\left[\frac{|B|^{2}+|C|^{2}}{\left|B h_{11}(1)+C h_{11}(2)\right|^{2}}\right]\right],
$$

where $B, C$ are functions of $h_{12}, h_{21}, h_{22}$ and the inner expectation is over $h_{11}(1)$ and $h_{11}(2)$ and outer expectation is over the rest of the variables. In (3.24)

$$
B h_{11}(1)+C h_{11}(2) \sim \mathcal{C N}\left(0,|B|^{2} P_{11}+|C|^{2} P_{11}\right)
$$

where $\mathbb{E}\left(\left|h_{11}(1)\right|^{2}\right)=\mathbb{E}\left(\left|h_{11}(2)\right|^{2}\right)=P_{11}, \quad B=h_{22}(1) h_{21}(2) h_{12}(2)$ and $C=$ $-h_{21}(1) h_{12}(1) h_{22}(2)$. Thus,

$$
\begin{aligned}
|\Delta|^{2} & =\left|B h_{11}(1)+C h_{11}(2)\right|^{2} \\
& =P_{11}\left(|B|^{2}+|C|^{2}\right) X_{11},
\end{aligned}
$$

where $X_{11}$ is a unit mean exponential variable ${ }^{1}$. Using (3.26) and (3.24), we have

$$
\begin{aligned}
T & =\mathbb{E}\left[\frac{|B|^{2}+|C|^{2}}{P_{11}\left(|B|^{2}+|C|^{2}\right) X_{11}}\right] \\
& =\mathbb{E}\left[\frac{1}{P_{11} X_{11}}\right] \rightarrow \infty
\end{aligned}
$$

since $\mathbb{E}\left(1 / X_{11}\right)=\int_{0}^{\infty} \frac{1}{x} e^{-x} d x \rightarrow \infty$.

The above analysis shows that even when perfect CSI is assumed, the mean value of the noise power is infinite.

Finally substituting (3.26) into (3.20), we have the instantaneous derivation of SNR given by

$$
\mathrm{SNR}_{\text {inst }_{x_{1,1}}}=\frac{\mathbb{E}\left[\left|x_{1,1}\right|^{2}\right]}{\left(\frac{\sigma^{2}}{P_{11} X_{11}}\right)},
$$

which is an exponential random variable with mean $\mathbb{E}\left[\left|x_{1,1}\right|\right]^{2} \frac{P_{11}}{\sigma^{2}}$.

\section{SINR for Imperfect CSI}

As shown in Fig. 3.4, the transmitter sends symbols to the relay via erroneous precoding vectors which will result in misalignment. We now analyze the SINR for the symbol $x_{1,1}$ at relay 1 for CSI error modelled by (2.23). The received vector $\mathbf{y}_{R_{1}}$ is then given by

$$
\mathbf{y}_{R_{1}}=\mathbf{H}_{11}\left(\hat{\mathbf{v}}_{1,1} x_{1,1}+\hat{\mathbf{v}}_{1,2} x_{1,2}\right)+\mathbf{H}_{21} \hat{\mathbf{v}}_{2,1} x_{2,1}+\mathbf{n}_{1}^{[1]}
$$

\footnotetext{
${ }^{1}$ Squared magnitude of a complex Gaussian (i.e. $\left.\left(\left|h_{11}(1)\right|^{2}\right)\right)$ is exponential.
} 


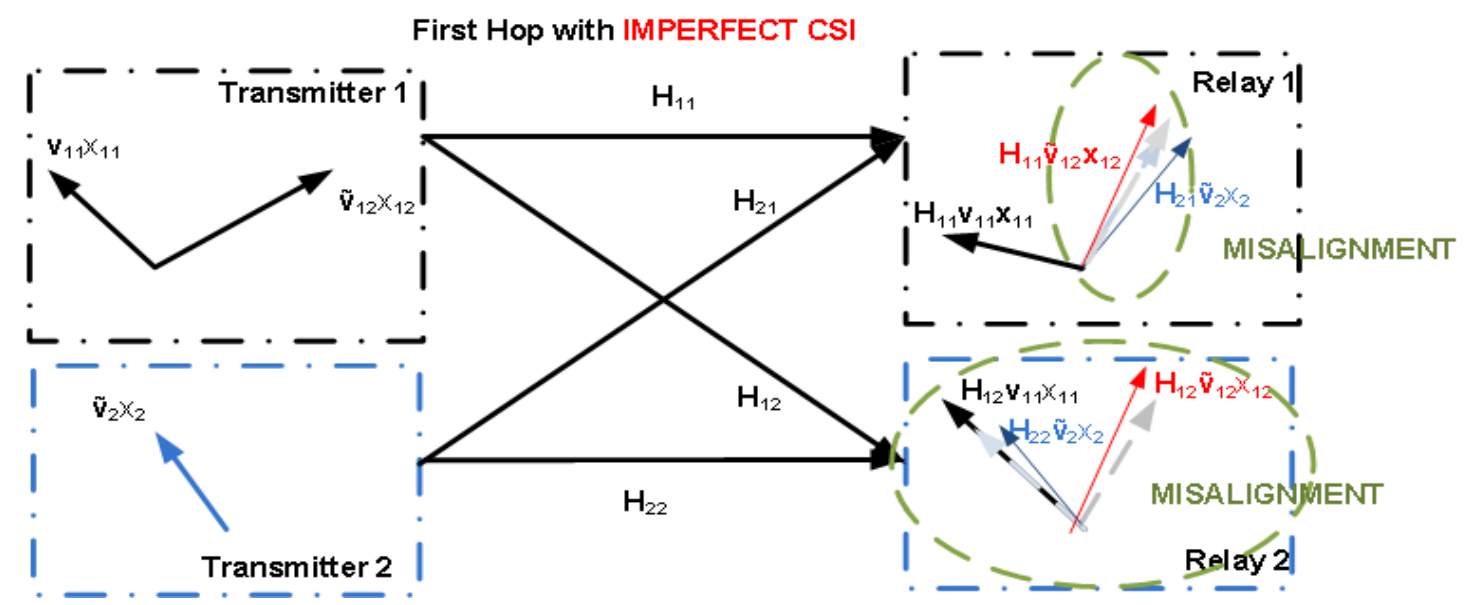

Figure 3.4: Misalignment due to the CSI errors in the first hop of [2]

where errored beamforming vectors are given by:

$$
\begin{aligned}
\hat{\mathbf{v}}_{1,1} & =\mathbf{v}_{1,1}=[1,1]^{T} \\
\hat{\mathbf{v}}_{2,1} & =\hat{\mathbf{H}}_{22}^{-1} \hat{\mathbf{H}}_{12} \mathbf{v}_{1,1} \\
\hat{\mathbf{v}}_{1,2} & =\hat{\mathbf{H}}_{11}^{-1} \hat{\mathbf{H}}_{21} \hat{\mathbf{H}}_{22}^{-1} \hat{\mathbf{H}}_{12} \mathbf{v}_{1,1} .
\end{aligned}
$$

Using (2.23), we can express the inverse of $\hat{\mathbf{H}}_{k j}$ by

$$
\begin{aligned}
\hat{\mathbf{H}}_{k j}^{-1} & =\left(\varsigma \mathbf{H}_{k j}+\bar{\varsigma} \boldsymbol{\Pi}_{k j}\right)^{-1} \\
& =\left(\varsigma \mathbf{H}_{k j}\left(\mathbf{I}+\frac{\bar{\varsigma}}{\varsigma} \mathbf{H}_{k j}^{-1} \boldsymbol{\Pi}_{k j}\right)\right)^{-1} \\
& \cong \frac{1}{\varsigma} \mathbf{H}_{k j}^{-1}-\frac{\bar{\varsigma}}{\varsigma^{2}} \mathbf{H}_{k j}^{-1} \boldsymbol{\Pi}_{k j} \mathbf{H}_{k j}^{-1},
\end{aligned}
$$

where we have neglected the second order terms $\left(\frac{\bar{\varsigma}}{\varsigma}\right)^{2}$ following (3.35). Considering that $\varsigma$ is larger than 0.90 in practical systems, $\left(\frac{\bar{\varsigma}}{\varsigma}\right)^{2}$ is indeed a small value. Note that substituting (3.36) into (3.3) and (3.4) gives the erroneous precoding vectors

$$
\begin{aligned}
\hat{\mathbf{v}}_{21} & =\left(\frac{1}{\varsigma} \mathbf{H}_{22}^{-1}-\frac{\bar{\varsigma}}{\varsigma^{2}} \mathbf{H}_{22}^{-1} \boldsymbol{\Pi}_{22} \mathbf{H}_{22}^{-1}\right)\left(\varsigma \mathbf{H}_{12}+{ }_{\varsigma} \boldsymbol{\Pi}_{12}\right) \mathbf{v}_{1,1} \\
& =\left(\mathbf{H}_{22}^{-1} \mathbf{H}_{12}+\frac{\bar{\varsigma}}{\varsigma} \mathbf{H}_{22}^{-1} \boldsymbol{\Pi}_{12}-\frac{\bar{\varsigma}}{\varsigma} \mathbf{H}_{22}^{-1} \boldsymbol{\Pi}_{22} \mathbf{H}_{22}^{-1} \mathbf{H}_{12}\right) \mathbf{v}_{1,1},
\end{aligned}
$$


and

$$
\begin{aligned}
\hat{\mathbf{v}}_{12} & =\left(\frac{1}{\varsigma} \mathbf{H}_{11}^{-1}-\frac{\bar{\varsigma}}{\varsigma^{2}} \mathbf{H}_{11}^{-1} \boldsymbol{\Pi}_{11} \mathbf{H}_{11}^{-1}\right)\left(\varsigma \mathbf{H}_{21}+\bar{\varsigma} \mathbf{\Pi}_{21}\right) \\
& \left(\frac{1}{\varsigma} \mathbf{H}_{22}^{-1}-\frac{\bar{\varsigma}}{\varsigma^{2}} \mathbf{H}_{22}^{-1} \boldsymbol{\Pi}_{22} \mathbf{H}_{22}^{-1}\right)\left(\varsigma \mathbf{H}_{12}+\bar{\varsigma} \boldsymbol{\Pi}_{12}\right) \mathbf{v}_{1,1} \\
& =\left(\left(\mathbf{H}_{11}^{-1} \mathbf{H}_{21} \mathbf{H}_{22}^{-1} \mathbf{H}_{12}-\frac{\bar{\varsigma}}{\varsigma} \mathbf{H}_{11}^{-1} \boldsymbol{\Pi}_{11} \mathbf{H}_{11}^{-1} \mathbf{H}_{21} \mathbf{H}_{22}^{-1} \mathbf{H}_{12}\right)\right. \\
& \left.+\left(\frac{\bar{\varsigma}}{\varsigma} \boldsymbol{\Pi}_{21} \mathbf{H}_{11}^{-1} \mathbf{H}_{22}^{-1} \mathbf{H}_{12}-\frac{\bar{\varsigma}}{\varsigma} \mathbf{H}_{22}^{-1} \mathbf{\Pi}_{22} \mathbf{H}_{22}^{-1} \mathbf{H}_{11}^{-1} \mathbf{H}_{21} \mathbf{H}_{12}+\frac{{ }_{\varsigma}}{\varsigma} \mathbf{\Pi}_{12} \mathbf{H}_{11}^{-1} \mathbf{H}_{21} \mathbf{H}_{22}^{-1}\right)\right) \mathbf{v}_{11}
\end{aligned}
$$

where we neglected the terms which have orders of $\left(\frac{\bar{\varsigma}}{\varsigma}\right)^{2}$ and above. Denote $\hat{\mathbf{v}}_{21}, \hat{\mathbf{v}}_{12}$ by

$$
\begin{aligned}
\hat{\mathbf{v}}_{21} & =\left(\mathbf{H}_{22}^{-1} \mathbf{H}_{12}+\boldsymbol{\Theta}_{12}\right) \mathbf{v}_{11} \\
\hat{\mathbf{v}}_{12} & =\left(\mathbf{H}_{11}^{-1} \mathbf{H}_{21} \mathbf{H}_{22}^{-1} \mathbf{H}_{12}+\boldsymbol{\Theta}_{21}\right) \mathbf{v}_{11},
\end{aligned}
$$

where

$$
\begin{aligned}
& \boldsymbol{\Theta}_{12}=\mathbf{A} \Pi_{12}+\mathbf{B} \Pi_{22} \\
& \boldsymbol{\Theta}_{21}=\mathbf{C \Pi}_{11}+\mathbf{D} \Pi_{21}+\mathbf{F} \Pi_{22}+\Psi \Pi_{12},
\end{aligned}
$$

and

$$
\begin{aligned}
& \mathbf{A}={ }_{\varsigma}^{\bar{\varsigma}} \mathbf{H}_{22}^{-1} \quad \mathbf{B}=-\frac{\bar{\varsigma}}{\varsigma} \mathbf{H}_{22}^{-2} \mathbf{H}_{12} \\
& \mathbf{C}=-{ }_{\varsigma}^{\bar{\varsigma}} \mathbf{H}_{11}^{-2} \mathbf{H}_{21} \mathbf{H}_{22}^{-1} \mathbf{H}_{12} \quad \mathbf{D}=\frac{{ }_{\varsigma}}{\varsigma} \mathbf{H}_{11}^{-1} \mathbf{H}_{22}^{-1} \mathbf{H}_{12} \\
& \mathbf{F}=-\frac{\bar{\varsigma}}{\varsigma} \mathbf{H}_{22}^{-2} \mathbf{H}_{11}^{-1} \mathbf{H}_{21} \mathbf{H}_{12} \quad \boldsymbol{\Psi}=\frac{\bar{\varsigma}}{\varsigma} \mathbf{H}_{11}^{-1} \mathbf{H}_{21} \mathbf{H}_{22}^{-1} .
\end{aligned}
$$

Then we can rewrite (3.30)

$$
\begin{aligned}
\hat{\mathbf{y}}_{R 1} & =\mathbf{H}_{11}\left(\mathbf{v}_{1,1} x_{1,1}+\left(\mathbf{H}_{11}^{-1} \mathbf{H}_{21} \mathbf{H}_{22}^{-1} \mathbf{H}_{12}+\boldsymbol{\Theta}_{21}\right) \mathbf{v}_{1,1} x_{1,2}\right) \\
& +\mathbf{H}_{21}\left(\mathbf{H}_{22}^{-1} \mathbf{H}_{12}+\boldsymbol{\Theta}_{12}\right) \mathbf{v}_{1,1} x_{2,1}+\mathbf{n}_{1}^{[1]} \\
& =\mathbf{H}_{11} \mathbf{v}_{1,1} x_{1,1}+\mathbf{H}_{11} \mathbf{v}_{1,2} x_{1,2}+\mathbf{H}_{11} \boldsymbol{\Theta}_{21} \mathbf{v}_{1,1} x_{1,2}+\mathbf{H}_{11} \mathbf{v}_{1,2} x_{2,1} \\
& +\mathbf{H}_{21} \boldsymbol{\Theta}_{12} \mathbf{v}_{1,1} x_{2,1}+\mathbf{n}_{1}^{[1]} \\
& =\mathbf{H}_{11} \mathbf{v}_{1,1} x_{1,1}+\mathbf{H}_{11} \mathbf{v}_{1,2}\left(x_{1,2}+x_{2,1}\right)+\mathbf{H}_{11} \boldsymbol{\Theta}_{21} \mathbf{v}_{1,1} x_{1,2}+\mathbf{H}_{21} \Theta_{12} \mathbf{v}_{1,1} x_{2,1}+\mathbf{n}_{1}^{[1]} .
\end{aligned}
$$


After processing the received signal in (3.48) with $\mathrm{ZF}$ matrix, $\mathbf{H}_{R_{1}}^{-1}$, we get,

$$
\begin{aligned}
\hat{\mathbf{x}}_{R_{1}} & =\left[\begin{array}{c}
\hat{x}_{R_{1}, 1} \\
\hat{x}_{R_{1}, 2}
\end{array}\right] \\
& =\mathbf{H}_{R_{1}}^{-1} \hat{\mathbf{y}}_{R_{1}}=\left[\begin{array}{c}
x_{1,1} \\
x_{1,2}+x_{2,1}
\end{array}\right]+\mathbf{H}_{R_{1}}^{-1}\left(\mathbf{H}_{11} \Theta_{21} \mathbf{v}_{1,1} x_{1,2}+\mathbf{H}_{21} \Theta_{12} \mathbf{v}_{1,1} x_{2,1}+\mathbf{n}_{1}^{[1]}\right) .
\end{aligned}
$$

Using the computation of (3.49) and following a similar approach to equation (3.19), one can show that

$$
\begin{aligned}
\mathbb{E}\left[\hat{\mathbf{x}}_{R_{1}} \hat{\mathbf{x}}_{R_{1}}^{*}\right] & =\left[\begin{array}{cc}
\mathbb{E}\left|x_{1,1}\right|^{2} & 0 \\
0 & \mathbb{E}\left|x_{1,2}\right|^{2}+\mathbb{E}\left|x_{2,1}\right|^{2}
\end{array}\right] \\
& +\mathbb{E}\left[\mathbf{H}_{R_{1}}^{-1} \boldsymbol{\Omega} \mathbf{\Omega}^{*} \mathbf{H}_{R_{1}}^{-1 *}\right]
\end{aligned}
$$

where $\boldsymbol{\Omega}=\mathbf{H}_{11} \boldsymbol{\Theta}_{21} \mathbf{v}_{1,1} x_{1,2}+\mathbf{H}_{21} \boldsymbol{\Theta}_{12} \mathbf{v}_{1,1} x_{2,1}+\mathbf{n}_{1}^{[1]}$.

After some manupilation to the additional interference and noise term of (3.50), $\boldsymbol{\Lambda}=\left(\mathbb{E}\left[\mathbf{H}_{R_{1}}^{-1} \boldsymbol{\Omega} \boldsymbol{\Omega}^{*} \mathbf{H}_{R_{1}}^{-1 *}\right]\right)$, one can show that the instantaneous interference and noise at relay 1 which is given by

$$
\begin{aligned}
\boldsymbol{\Lambda} & =\mathbf{H}_{R_{1}}^{-1}\left[\mathbb{E}\left|x_{1,2}\right|^{2} \mathbf{H}_{11} \mathbf{T} \mathbf{H}_{11}^{*}\right. \\
& \left.+\mathbb{E}\left|x_{2,1}\right|^{2} \mathbf{H}_{21} \mathbf{\Upsilon} \mathbf{H}_{21}^{*}+\sigma^{2} \mathbf{I}\right] \mathbf{H}_{R_{1}}^{-1 *}
\end{aligned}
$$

where

$$
\begin{aligned}
& \mathbf{T}=P_{11} \mathbf{C C}^{*}+P_{21} \mathbf{D D}^{*}+P_{22} \mathbf{F} \mathbf{F}^{*}+P_{12} \boldsymbol{\Psi} \Psi^{*} \\
& \mathbf{\Upsilon}=P_{21} \mathbf{A A}^{*}+P_{22} \mathbf{B B}^{*}
\end{aligned}
$$

and

$$
\begin{aligned}
& P_{11}=\mathbb{E}\left(\left|h_{11}(1)\right|^{2}\right)=\mathbb{E}\left(\left|h_{11}(2)\right|^{2}\right) \\
& P_{12}=\mathbb{E}\left(\left|h_{12}(1)\right|^{2}\right)=\mathbb{E}\left(\left|h_{12}(2)\right|^{2}\right) \\
& P_{21}=\mathbb{E}\left(\left|h_{21}(1)\right|^{2}\right)=\mathbb{E}\left(\left|h_{21}(2)\right|^{2}\right) \\
& P_{22}=\mathbb{E}\left(\left|h_{22}(1)\right|^{2}\right)=\mathbb{E}\left(\left|h_{22}(2)\right|^{2}\right) .
\end{aligned}
$$

Finally, instantenous SINR is given for the symbol $x_{1,1}$ by

$$
\operatorname{SINR}_{\text {inst }_{x_{1,1}}}=\frac{\mathbb{E}\left|x_{1,1}\right|^{2}}{\boldsymbol{\Lambda}(1,1)},
$$

where $\boldsymbol{\Lambda}(1,1)$ is the first entry of the matrix $\boldsymbol{\Lambda}$ and denotes the additional interference and noise for the symbol $x_{1,1}$. 


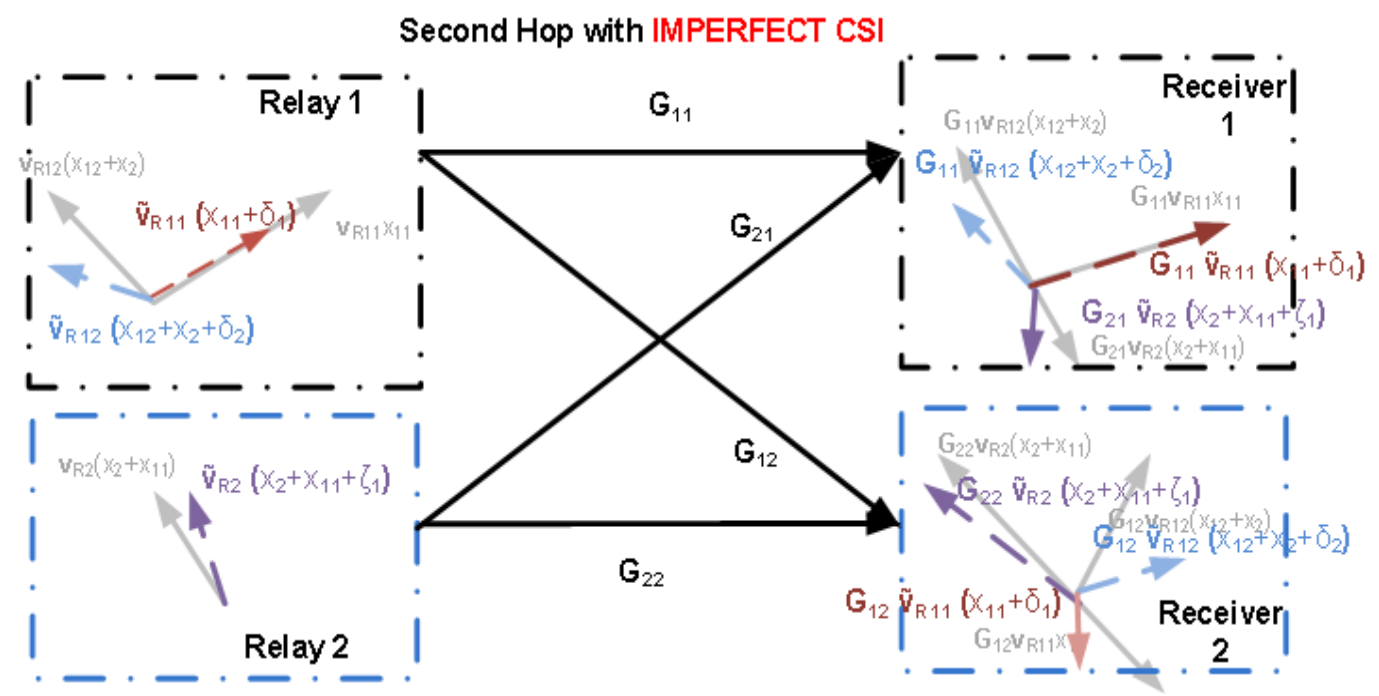

Figure 3.5: Misalignment due to the CSI errors in the second hop of [2]

The first term in $\Lambda$ which is one of the interference terms due to imperfect CSI is given by

$$
\boldsymbol{\Lambda}_{1}=\mathbf{H}_{R_{1}} \mathbb{E}\left|x_{1,2}\right|^{2} \mathbf{H}_{11} P_{11} \mathbf{C C}^{*} \mathbf{H}_{11}^{*} \mathbf{H}_{R_{1}}^{-1 *} .
$$

One can show that $\lambda_{11}=\boldsymbol{\Lambda}_{1}(1,1)$ is given by

$$
\lambda_{11}=\frac{P_{11} \mathbb{E}\left|x_{1,2}\right|^{2}\left(\frac{\bar{\varsigma}}{\varsigma}\right)^{2} \Delta_{2}}{|\Delta|^{2}},
$$

where

$$
\Delta_{2}=\left(\frac{1}{\left|h_{11}(1)\right|^{2}}+\frac{1}{\left|h_{11}(2)\right|^{2}}\right) \tau
$$

and $\tau=\left|h_{12}(2)\right|^{2}\left|h_{21}(2)\right|^{2}\left|h_{12}(1)\right|^{2}\left|h_{21}(1)\right|^{2}$. Additional terms in (3.59) follow similar structure. Noting that $|\Delta|^{2}$ is defined in (3.26) and includes the unit norm exponential term $X_{11}$, we observe that each of the interference terms will have infinite mean, thus resulting in severe degradation in SINR at the relay.

\section{End-To-End Interference with Imperfect CSI}

Having derived the SINR at the relay stage, it is straightforward to show that end-to-end interference at the destination can be expressed as the sum of the individual interference components arising from each hop. Without loss of generality, we consider $M=2$. From (3.11) and (3.12), we have that the signal at relay 1 is

$$
\mathbf{x}_{R_{1}}=\left[\begin{array}{c}
x_{1,1} \\
x_{1,2}+x_{2,1}
\end{array}\right]+\left[\begin{array}{l}
\delta_{1} \\
\delta_{2}
\end{array}\right]
$$


and similarly at relay 2

$$
\mathbf{x}_{R_{2}}=\left[\begin{array}{c}
x_{1,1}+x_{2,1} \\
x_{1,2}
\end{array}\right]+\left[\begin{array}{l}
\zeta_{1} \\
\zeta_{2}
\end{array}\right]
$$

where $\delta_{1}, \delta_{2}$ and $\zeta_{1}, \zeta_{2}$ are the additive interference and noise terms arising from the first hop. Then relay 1 sends $x_{1,1}+\delta_{1}$ and $x_{1,2}+x_{2,1}+\delta_{2}$ along the erroneous relay precoding vectors $\hat{\mathbf{v}}_{R_{1}, 1}$ and $\hat{\mathbf{v}}_{R_{1}, 2}$ respectively as shown in Fig. 3.5. Similarly the relay 2 sends $x_{1,1}+x_{2,1}+\zeta_{1}$ along the erroneous relay precoding vector $\hat{\mathbf{v}}_{R_{2}}$. In Fig. 3.5, alignment with the perfect precoders is show with light-gray colour, whereas the dimensions of the signals sent along the erroneous precoders are shown with colourful arrows. Thus, the misalignment could be noticed due to the erroneous precoders. Using an expansion for $\hat{\mathbf{G}}_{j n}^{-1}$ similar to (3.36), erroneous relay precoding vectors can be derived as

$$
\begin{aligned}
\hat{\mathbf{v}}_{R_{1}, 1} & =\mathbf{v}_{R_{1}, 1}=[1,1]^{T} \\
\hat{\mathbf{v}}_{R_{1}, 1} & =-\hat{\mathbf{G}}_{22}^{-1} \hat{\mathbf{G}}_{12} \mathbf{v}_{R_{1}, 1} \approx \mathbf{v}_{R_{2}, 1}+\epsilon_{1} \\
\hat{\mathbf{v}}_{R_{1}, 2} & =\hat{\mathbf{G}}_{11}^{-1} \hat{\mathbf{G}}_{21} \hat{\mathbf{G}}_{22}^{-1} \hat{\mathbf{G}}_{12} \mathbf{v}_{R_{1}, 1} \approx \mathbf{v}_{R_{1}, 2}+\epsilon_{2},
\end{aligned}
$$

where the error components, $\epsilon_{1}$ and $\epsilon_{2}$ can be derived replacing $\mathbf{H}_{k j}$ channel coefficients with $\mathbf{G}_{k j}$ channel coefficients in (3.36). Because $M-1$ symbols are sent from the second relay, the second term is not relayed. Simple substitution of (3.62) into (3.15) gives

$$
\begin{aligned}
\hat{\mathbf{y}}_{1} & =\mathbf{G}_{11}\left[\hat{\mathbf{v}}_{R_{1}, 1}\left(x_{1,1}+\delta_{1}\right)+\hat{\mathbf{v}}_{R_{1}, 2}\left(x_{1,2}+x_{2,1}+\delta_{2}\right)\right] \\
& +\mathbf{G}_{21} \hat{\mathbf{v}}_{R_{2}, 1}\left(x_{1,1}+x_{2,1}+\zeta_{1}\right)+\mathbf{n}_{1}^{[2]}
\end{aligned}
$$

where $\mathbf{n}_{1}^{[2]}$ is the additive white Gaussian noise in the second hop. Using (3.65) and (3.66),

$$
\begin{aligned}
\hat{\mathbf{y}}_{1} & \left.=\mathbf{G}_{11} \mathbf{v}_{R_{1}, 1}\left(x_{1,1}+\delta_{1}\right)+\mathbf{v}_{R_{1}, 2}\left(x_{1,2}+x_{2,1}+\delta_{2}\right)\right] \\
& +\mathbf{G}_{21} \mathbf{v}_{R_{2}, 1}\left(x_{1,1}+x_{2,1}+\zeta_{1}\right)+\tilde{\mathbf{n}}_{1}^{[2]}
\end{aligned}
$$

where $\tilde{\mathbf{n}}_{1}^{[2]}$ is the combined noise and interference term which includes interference arising from the error components of the precoding vectors $\epsilon_{1}$ and $\epsilon_{2}$, in the second hop. For example, considering (3.68), the interference that $x_{1,1}$ suffers from is the interference arising from the first hop, $\delta_{1}$, and also the second hop interference which is included in $\tilde{\mathbf{n}}_{1}^{[2]}$. Similar analysis can be applied to destination 2. Thus, we have shown that, under the approximation in (3.36), destination receives the symbol $\hat{x}_{1,1}$ corrupted by additive interference and noise 
due to CSI errors.

\subsubsection{Using Multiple Antennas instead of Symbol Exten- sion}

The authors of [2] implemented IA into a multihop network using symbol extension for a SISO system in order to achieve 2 DoF asymptotically. However, the scheme proposed for SISO system with $M$ symbol extensions can be directly carried over to the $N_{t} \times N_{r}$ MIMO case where $N_{t}=N_{r}=M$ now denotes the number of antenna at each transmitter, relay and receiver, instead of symbol extension in [2]. The difference for the MIMO case is that the channels are not in a diagonal form such that

$$
\mathbf{H}_{k j}=\left[\begin{array}{cccc}
h_{k j}(1,1) & h_{k j}(1,2) & \cdots & h_{k j}(1, M) \\
h_{k j}(2,1) & h_{k j}(2,2) & \cdots & h_{k j}(2, M) \\
\vdots & \vdots & \ddots & \vdots \\
h_{k j}(M, 1) & h_{k j}(M, 2) & \cdots & h_{k j}(M, M)
\end{array}\right]
$$

and similarly for the channels in the second hop as

$$
\mathbf{G}_{j k}=\left[\begin{array}{cccc}
g_{k j}(1,1) & g_{k j}(1,2) & \cdots & g_{k j}(1, M) \\
g_{k j}(2,1) & g_{k j}(2,2) & \cdots & g_{k j}(2, M) \\
\vdots & \vdots & \ddots & \vdots \\
g_{k j}(M, 1) & g_{k j}(M, 2) & \cdots & g_{k j}(M, M)
\end{array}\right]
$$

Hence, the erronous matrix, $\boldsymbol{\Pi}$, in $(2.23)$ can not be a diagonal matrix either. Also one can note that the extra dimensionality required from symbols in time domain can be obtained via increased antenna numbers in space domain.

\subsubsection{Simulation Results}

In Figure 3.6, we plot the CDFs of SNR and SINR for symbol $x_{1,1}$ at relay 1 using the equations (3.29) and (3.58). The figure demonstrates the degradation of SINR due to the increasing $\varsigma$ in (2.23), as expected. 


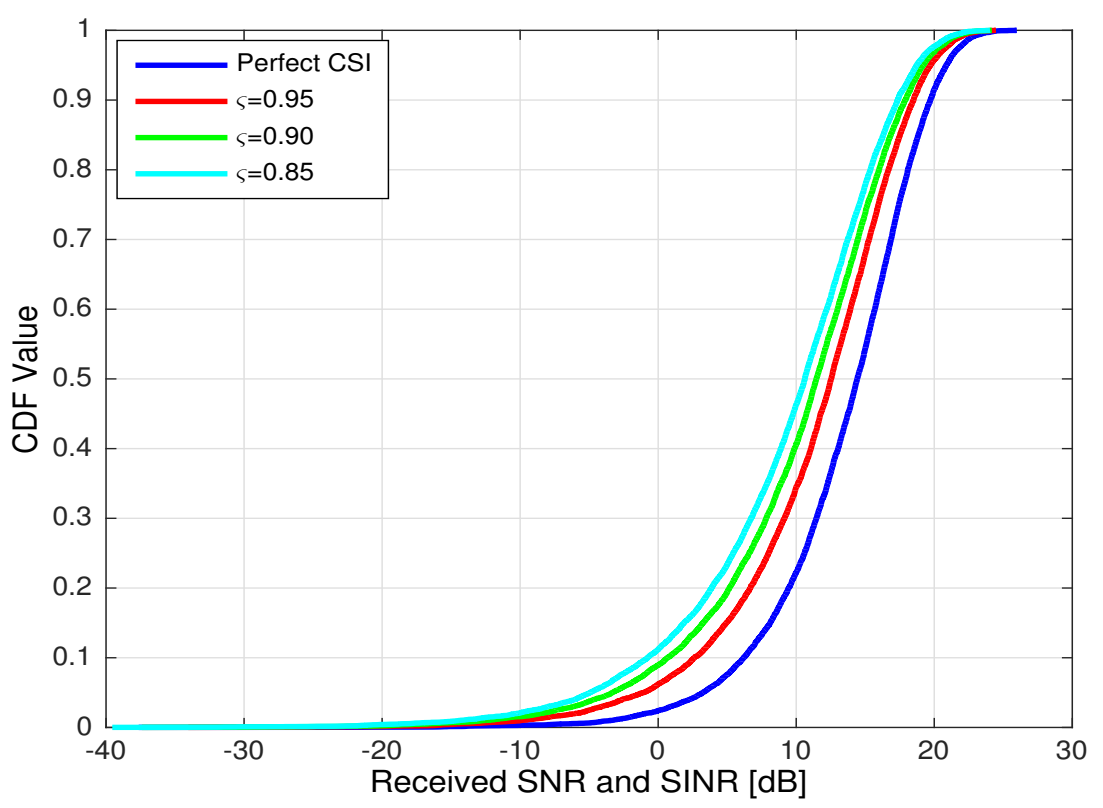

Figure 3.6: CDF of SNR with perfect CSI and SINR with imperfect CSI for $x_{1,1}$ at relay 1 .

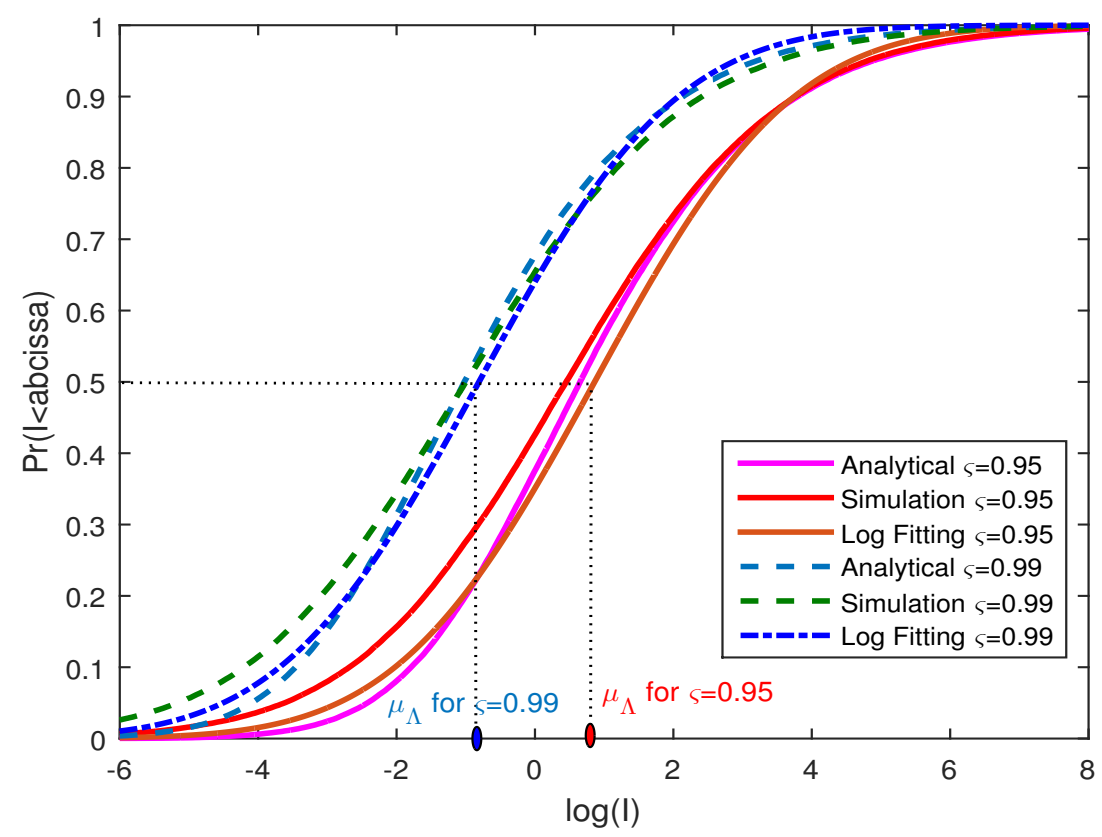

Figure 3.7: CDF of interference at relay 1 for $x_{1,1}$ via simulations, analytical analysis and log normal fitting

In Figure 3.7, we investigate the CDF of the interference term for the symbol $x_{1,1}$ at Relay $1, \boldsymbol{\Lambda}(1,1)$, using the equation (3.51) without noise $(\sigma=0)$. Analytical results agree closely to simulation results with the discrepancy resulting 
from neglecting $\left(\frac{\bar{\varsigma}}{\varsigma}\right)^{2}$ terms which increase with decreasing $\varsigma$. Note that the simulation results in Figure 3.7 also include the effect of higher order terms which are neglected in (3.36) and therefore (3.51). This difference between analytical and simulation results is more visible for lower interference and also smaller $\varsigma$ values. We also showed that the interference is closely modelled by a log normal distribution, $\ln \mathcal{N}\left(\mu_{\Lambda}, \sigma_{\Lambda}^{2}\right)$, where $\mu_{\Lambda}, \sigma_{\Lambda}^{2}$ are the mean and variance of $\boldsymbol{\Lambda}(1,1)$, respectively. The log normal distribution can be defined as a continuous probability distribution of a random variable whose logarithm is normally distributed. Because of the prominent tails of a log normal distributions, the large instantaneous interference degrades the system performance drastically (See Figure $3.9)$.

In Figure 3.8, we simulate the end-to-end interference values as discussed earlier in Section 3.1.2, where we assumed a feedback error while generating both transmitter and relay precoding vectors. As with the interference at the relay stage, the overall interference is shown to also follow a log normal distribution. In Figure 3.9, we investigate the bit error rates of each symbol for $M=2$ with QPSK modulation with respect to transmit $\mathrm{SNR}^{2}$. The channel at both hops is assumed to be complex Gaussian and we consider both perfect and imperfect CSI. For the imperfect CSI case $\varsigma=0.99$ is chosen. We note that $x_{1,1}, x_{1,2}$ and $x_{2}$ are corrupted by different noise levels as a result of aligning them into different dimensions during transmission and also due to the decoding technique. For example after decoding $x_{11}$ from the second dimension in order to decode $x_{12}$, the decision errors for $x_{11}$ impact decoding of $x_{12}$. It is also seen that bit error rate performances degrades drastically with as little estimation error as $\varsigma=0.99$. In order to show the loss due to multi hop and successive decoding, we also compare our results with analytical bit error rates of QPSK for 1 transmitter - 1 receiver system, under the assumption of complex gaussian fading channel (yellow curve with star) and AWGN channel (green curve with star).

In Figure 3.10, we investigate symbol error rates for receiver 1, considering different scenarios for channel estimation error. $\varsigma$ is assumed to be 0.99 and we consider three cases, error only at transmitter precoding vectors, error only at relay precoding vectors and third case is both transmitter and relay precoding vectors are erroneous. We note that the system performs worse when precoding vectors are erroneous only at transmitters, rather than only at relays. The reason is the fact that when there is error in transmitter side, the relay receives misaligned signals even though the relay has perfect CSI. Therefore the transmission is erroneous in both hops. However, if the transmitter has perfect CSI and erroneous precoders are only generated at relays, the transmission is

\footnotetext{
${ }^{2}$ Transmit SNR is the Signal-to Noise-Ratio of the transmitted symbol. It is calculated as the energy per bit to noise power at the receiver.
} 
erroneous only in the second hop, which provides better symbol error rates than the case where erroneous precoders are only generated at transmitters. On the other hand, the performance in the case of error at both precoding vectors at transmitters and relays is the worst as expected.

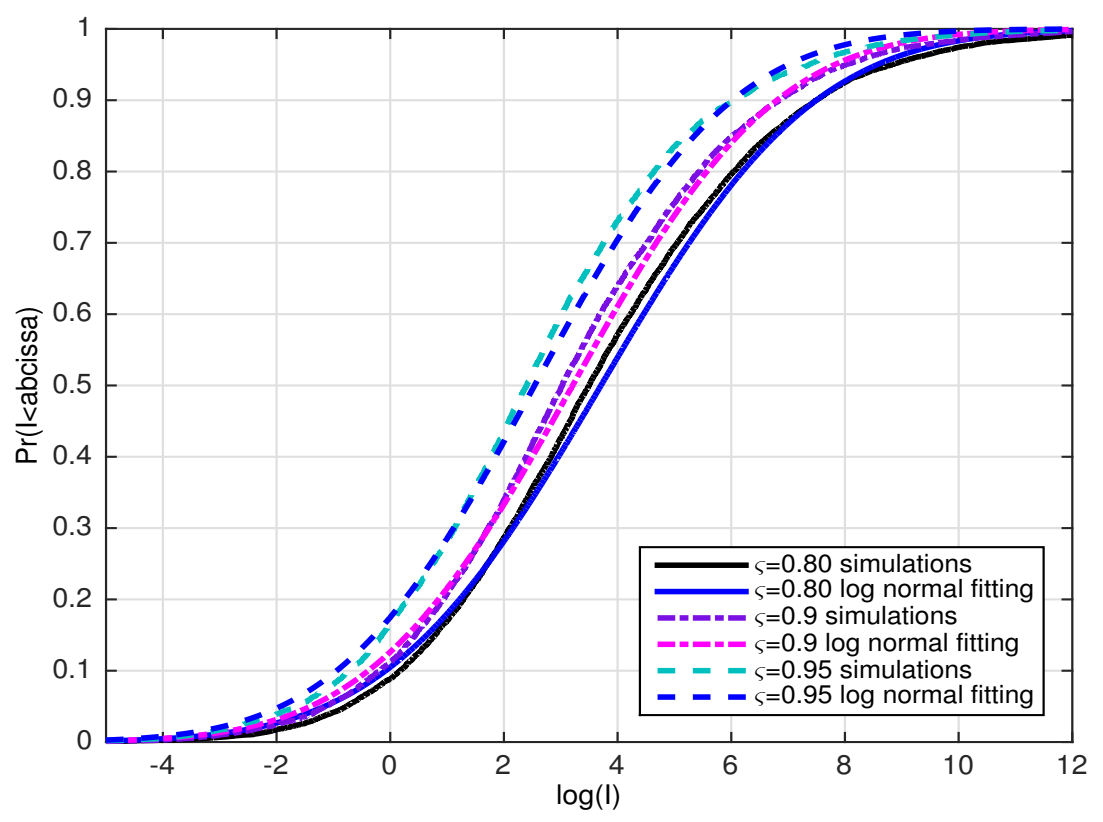

Figure 3.8: CDF of end-to-end Interference for $x_{1,1}$ symbol via simulations and log-normal fitting.

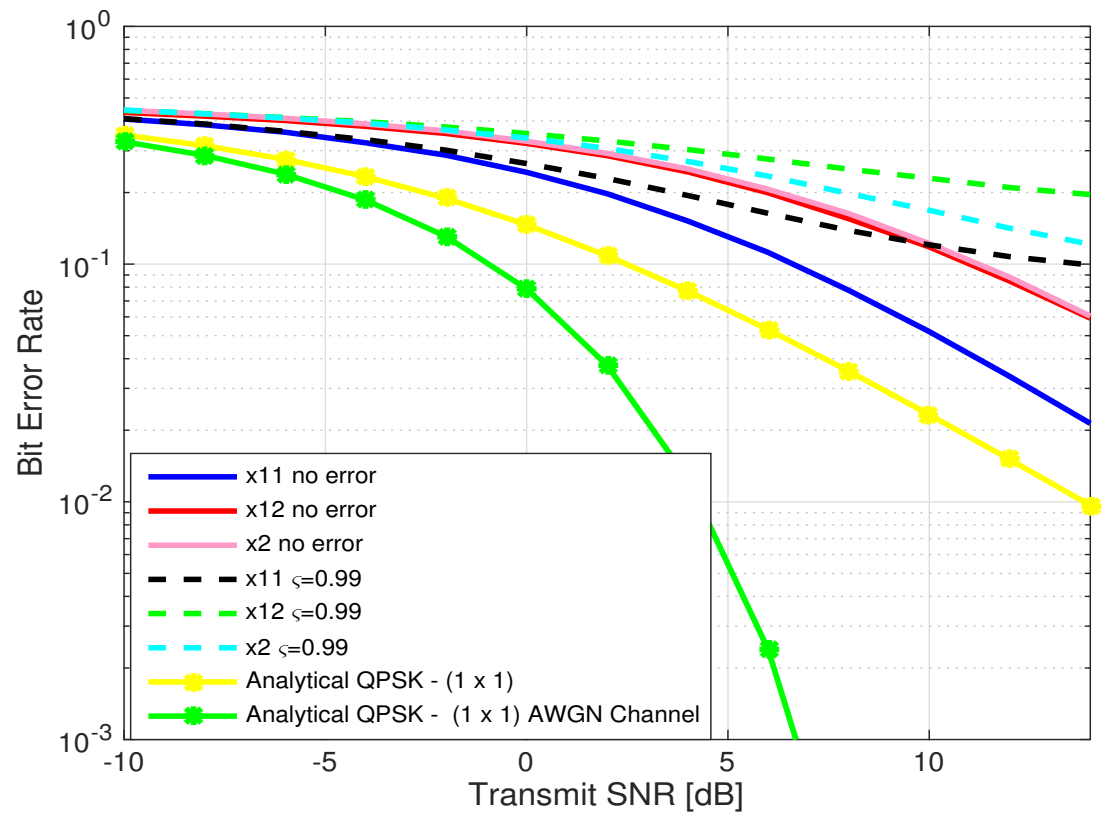

Figure 3.9: Bit error rates of each symbol with perfect and imperfect CSI cases. 


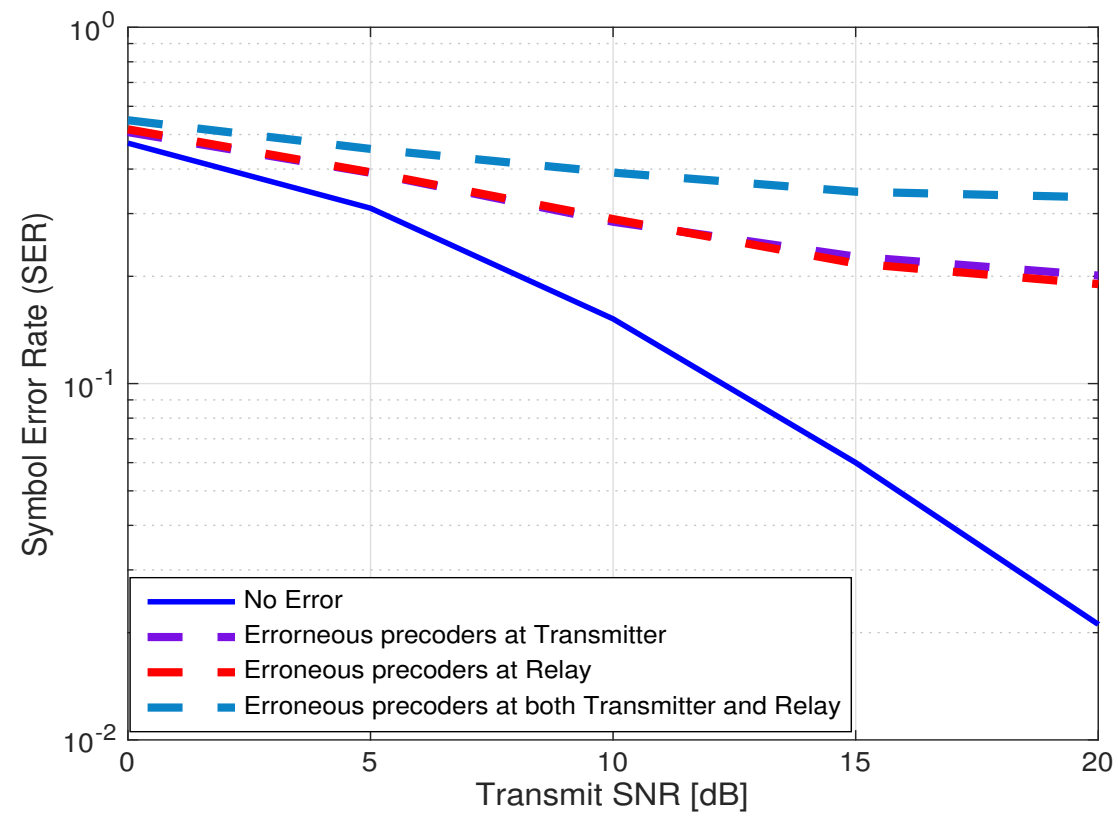

Figure 3.10: The symbol error rates for receiver 1 with channel estimation error: erroneous precoders at transmitters only, at relays only, at both transmitters and relays.

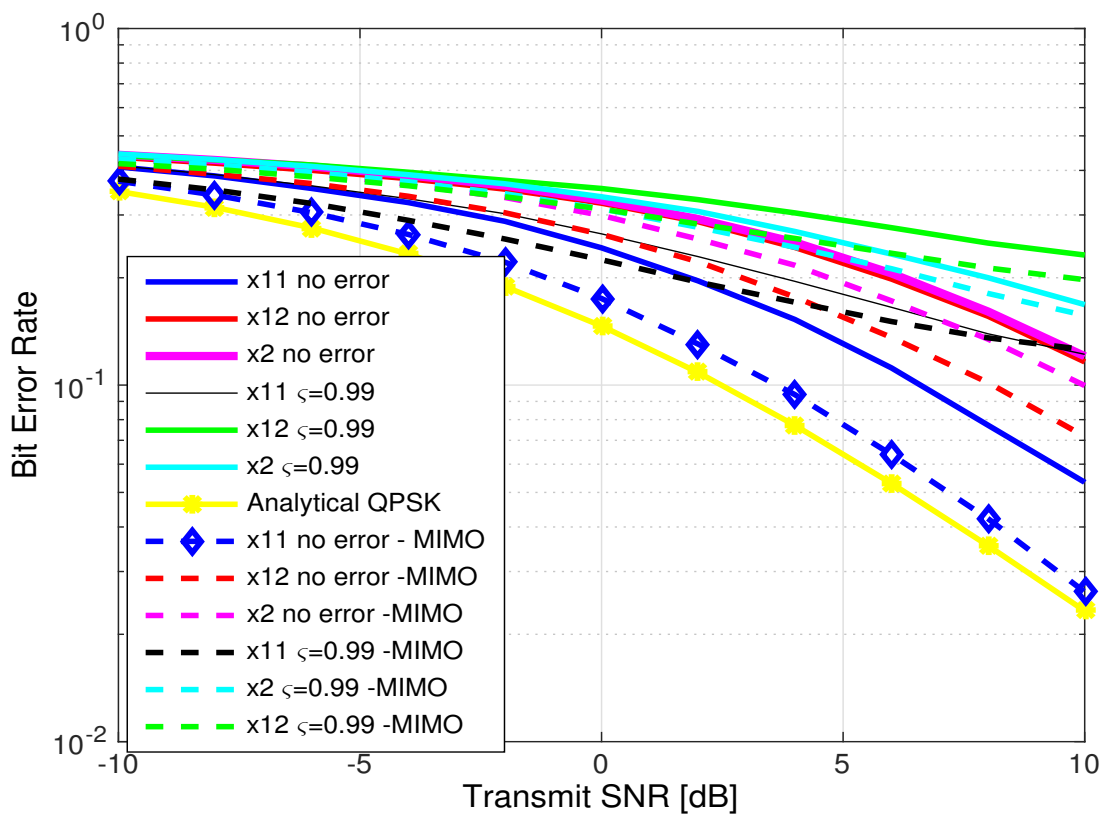

Figure 3.11: IA using symbol extension vs. MIMO, with perfect and imperfect CSI

Finally, we compare the BER results using symbol extension with employing multiple antennas. We assume that all transmitters relays and receivers have 
2 antennas, instead of 2 symbol extensions. Simulation results in Figure 3.11 present that employing multiple antennas provide better bit error rates (BER) compared to symbol extension for both perfect and imperfect CSI cases.

The reason why MIMO achieves better BER results is the fact that MIMO provides gain achieved by the diversity in the transmitters and the receivers and reduces the deep-fading effects of the wireless channel. This is because the diversity order of a $2 \times 2$ MIMO system is 4 , while the diversity order of a SISO system with 2 symbol extension is 2 .

\subsection{Summary}

In this chapter, we have discussed an IA scheme in multihop interference channels where relays are employed. We have given an alignment scheme for $2 \times 2 \times 2$ interference channels which consist of 2 transmitter 2 relays and 2 receivers. Furthermore we have considered imperfect CSI which cause misalignment of interference due to erroneous precoders. We have derived analytical expressions for the SNR and SINR at the relays. The interference caused by misalignment due to CSI errors is shown to be very prominent with the mean tending to infinity. We have shown that the interference due to CSI errors can be modelled via simulation by a log normal distribution. In conclusion, aligned interference neutralisation scheme for multihop relay networks is very prone to channel estimation errors that accumulate over time. This leads us to conclude that the gain proposed by the $2 \times 2 \times 2$ channel is only achievable in an ideal setting. The ZF receivers also contribute further to noise enhancement.

Considering results in this chapter, we will investigate how we can generate precoding vectors which could perform better with imperfect CSI. Furthermore, different kind of receivers instead of ZF can be implemented in order to avoid noise and interference enhancement. In the following chapters, we will address to these problems in cellular networks. 


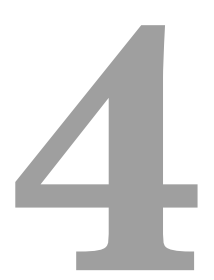

\section{Uplink Interference Alignment and Cancellation in Multicell Networks}

In this chapter, we discuss uplink IAC schemes for multi - user, multi - cellular networks. We begin with uplink 2-cell networks, followed by more complicated multicellular networks including heterogeneous networks. Here, we discuss various receiver types including ZF receivers, MMSE receivers and propose a novel receiver called the "Combined Receiver".

We can summarise some of the major contributions of this chapter which were published in [1] as follows

- We propose an IAC scheme with a novel receiver that we refer to as the Combined Receiver. The idea behind this receiver is to null the dominant interferers with ZF and then to use MMSE processing to reduce the effects of the remaining interference and noise.

- We implement our scheme in an IA heterogeneous uplink network which involves a mix of macro and pico cells.

- We show the range of remaining to dominant interference ratios in which our receiver outperforms the traditional MMSE receiver.

\subsection{Interference Alignment and Cancellation for Uplink 2-Cell Networks}

Here, we consider a two cell where each cell serves 2 users. Therefore, each BS receives four messages in total, two of which are the interference from the other 


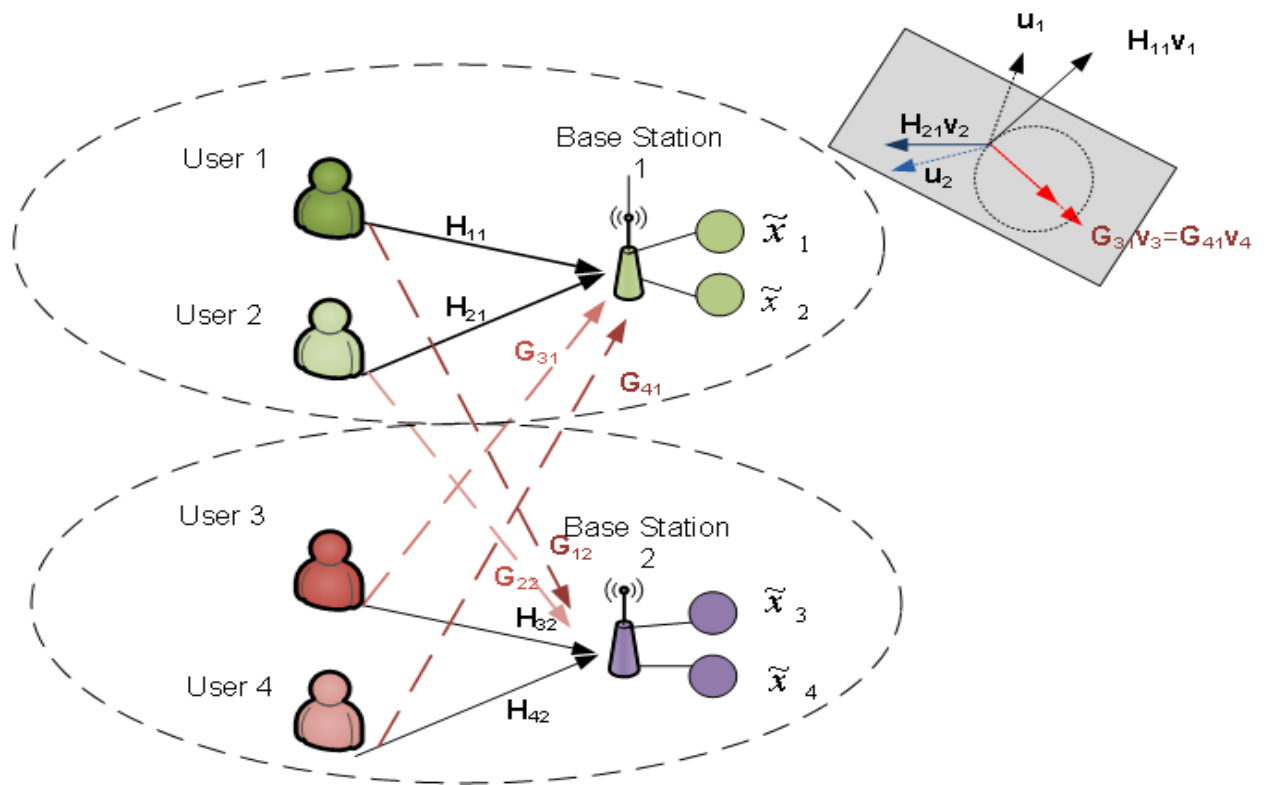

Figure 4.1: System model for 2 user-2 cell uplink MIMO system

cell users, which is called the intercell interference. We can summarise our work in this subsection as follows

- We reproduce and compare the results of [100] and [101].

- We evaluate the simulation results of such systems considering imperfect CSI and discuss its impact on the mean sum rates.

- We also use IEEE 802.16e codebooks which is given in Section 2.9 for comparing with the codebooks of [100] and [101].

Such a system is shown in Fig. 4.1. Each user is equipped with $N_{t}$ antennas, and each BS is equipped with $N_{r}$ antennas. User 1 and user 2 convey messages $x_{1}$ and $x_{2}$ to the BS 1 respectively, while user 3 and user 4 transmit their signals $x_{3}$ and $x_{4}$ to BS 2, respectively. The user $i$ transmits its message through a precoding vector $\mathbf{v}_{i}$ as follows

$$
\mathbf{x}_{i}=\mathbf{v}_{i} x_{i}
$$

The received signals at BS 1 and BS 2 can be represented as

$$
\begin{aligned}
& \mathbf{y}_{1}=\sum_{i=1}^{2} \mathbf{H}_{i 1} \mathbf{x}_{i}+\sum_{k=3}^{4} \mathbf{G}_{k 1} \mathbf{x}_{k}+\mathbf{n}_{1}, \\
& \mathbf{y}_{2}=\sum_{i=3}^{4} \mathbf{H}_{i 2} \mathbf{x}_{i}+\sum_{k=1}^{2} \mathbf{G}_{k 2} \mathbf{x}_{k}+\mathbf{n}_{2},
\end{aligned}
$$

where $\mathbf{n}_{j}$ is the AWGN vector at the BS $j$ and $\mathbf{H}_{i j} \in \mathcal{C N}(0,1),\left(N_{r} \times N_{t}\right)$ 
is the desired channel between the user $i$ and the BS $j$. Similarly, $\mathbf{G}_{i j} \in$ $\mathcal{C N}(0,1), \quad\left(N_{r} \times N_{t}\right)$ is the cross channel between the interfering user $i$ and the BS $j$. Each entry of the matrices is drawn from independently and identically distributed (i.i.d). All channel matrices in [100] are assumed to be full rank, which is also supported by the rank analysis of matrices in [102] and $\operatorname{rank}\left(\mathbf{H}_{i j}\right)=\operatorname{rank}\left(\mathbf{G}_{i j}\right)=\min \left(N_{r}, N_{t}\right)$.

Each BS decodes the desired messages coming from its serving users using postcoding vectors. Precoding and postcoding vectors are both generated at the BS (receiver). The BS then feeds back the users appropriate precoding vectors. The decoded signal for the user $j$ at BS 1 is represented as follows

$$
\tilde{x}_{j}=\mathbf{u}_{j}^{*}\left(\sum_{i=1}^{2} \mathbf{H}_{i 1} \mathbf{x}_{i}+\sum_{k=3}^{4} \mathbf{G}_{k 1} \mathbf{x}_{k}+\mathbf{n}_{1}\right) .
$$

Similarly the user $k$ at BS 2 can be decoded

$$
\tilde{x}_{k}=\mathbf{u}_{k}^{*}\left(\sum_{i=3}^{4} \mathbf{H}_{i 2} \mathbf{x}_{i}+\sum_{k=1}^{2} \mathbf{G}_{k 2} \mathbf{x}_{k}+\mathbf{n}_{2}\right)
$$

where $\mathbf{u}_{k}$ is the postcoding vector which is used to decode the message $x_{k}$ of the user $k$. The postcoding vectors have unit norm $\left\|\mathbf{u}_{k}\right\|=1$ and they cancel the interference which is aligned within a subspace. In [100], IA is achieved by precoding vectors which are carefully generated based on channel state information at transmitters. Let us denote the interference subspace for BS 1 as $\boldsymbol{\eta}_{1}^{\mathrm{ICI}}$ which should span the intercell interference on the directions of $\mathbf{G}_{31} \mathbf{v}_{3}$ and $\mathbf{G}_{41} \mathbf{v}_{4}$ as shown in Fig. 4.1. Therefore the alignment condition can be given by [100]

$$
\operatorname{span}\left(\boldsymbol{\eta}_{1}^{\text {ICI }}\right)=\operatorname{span}\left(\left[\begin{array}{lll}
\mathbf{G}_{31} \mathbf{v}_{3} & \mathbf{G}_{41} \mathbf{v}_{4}
\end{array}\right]\right)
$$

The condition can be satisfied with the following equation [100]

$$
\left[\begin{array}{ccc}
\mathbf{I}_{N_{r}} & -\mathbf{G}_{31} & \mathbf{0} \\
\mathbf{I}_{N_{r}} & \mathbf{0} & -\mathbf{G}_{41}
\end{array}\right]\left[\begin{array}{c}
\boldsymbol{\eta}_{1}^{I C I} \\
\tilde{\mathbf{v}}_{3} \\
\tilde{\mathbf{v}}_{4}
\end{array}\right]=\mathbf{0}
$$

Provided $\mathbf{G}_{31}$ and $\mathbf{G}_{41}$ are full rank, the alignment can be obtained with probability of 1 [100]. Then the precoding vectors are normalised to have unit norm as

$$
\begin{aligned}
& \mathbf{v}_{3}=\frac{\tilde{\mathbf{v}}_{3}}{\left\|\tilde{\mathbf{v}}_{3}\right\|} \\
& \mathbf{v}_{4}=\frac{\tilde{\mathbf{v}}_{4}}{\left\|\tilde{\mathbf{v}}_{4}\right\|} .
\end{aligned}
$$


Similarly we can align the interference at BS 2 in the direction of $\boldsymbol{\eta}_{2}^{I C I}$. We can generate the precoding vectors of $\mathbf{v}_{1}$ and $\mathbf{v}_{2}$ considering the following equation

$$
\left[\begin{array}{ccc}
\mathbf{I}_{N_{r}} & -\mathbf{G}_{12} & \mathbf{0} \\
\mathbf{I}_{N_{r}} & \mathbf{0} & -\mathbf{G}_{22}
\end{array}\right]\left[\begin{array}{c}
\boldsymbol{\eta}_{2}^{\text {ICI }} \\
\tilde{\mathbf{v}}_{1} \\
\tilde{\mathbf{v}}_{2}
\end{array}\right]=\mathbf{0}
$$

The precoding vectors $\mathbf{v}_{1}, \mathbf{v}_{2}$ are normalised to have unit norm as

$$
\begin{aligned}
& \mathbf{v}_{1}=\frac{\tilde{\mathbf{v}}_{1}}{\left\|\tilde{\mathbf{v}}_{1}\right\|} \\
& \mathbf{v}_{2}=\frac{\tilde{\mathbf{v}}_{2}}{\left\|\tilde{\mathbf{v}}_{2}\right\|} .
\end{aligned}
$$

After generating the precoding vectors, receiver $i$ can generate the postcoding vectors $\mathbf{u}_{i}$ which nulls out the interference subspace. For example, the BS 1 can generate postcoding vectors to decode $x_{1}$ and $x_{2}$ satisfying the following conditions [100]

$$
\begin{aligned}
& \mathbf{u}_{1}^{*}\left[\begin{array}{ll}
\mathbf{H}_{21} \mathbf{v}_{2} & \boldsymbol{\eta}_{1}^{\mathrm{ICI}}
\end{array}\right]=0 \\
& \mathbf{u}_{2}^{*}\left[\begin{array}{ll}
\mathbf{H}_{11} \mathbf{v}_{1} & \left.\boldsymbol{\eta}_{\mathbf{1}}^{\mathrm{ICI}}\right]
\end{array}\right]=0 .
\end{aligned}
$$

Similarly, the BS 2 can generate the postcoding vectors to decode $x_{3}$ and $x_{4}$ as

$$
\begin{aligned}
& \mathbf{u}_{3}^{*}\left[\begin{array}{ll}
\mathbf{H}_{42} \mathbf{v}_{4} & \boldsymbol{\eta}_{\mathbf{2}}^{\mathrm{ICI}}
\end{array}\right]=0 \\
& \mathbf{u}_{4}^{*}\left[\begin{array}{ll}
\mathbf{H}_{32} \mathbf{v}_{3} & \boldsymbol{\eta}_{\mathbf{2}}^{\mathrm{ICI}}
\end{array}\right]=0 \text {. }
\end{aligned}
$$

These postcoding vectors which null out the interference space are known as ZF postcoders. As a result, 1 degree of freedom per user can be achieved when $N_{r}=3$ and $N_{t}=2$. The achievable rate for user $i$ at BS 1 is given in [100] as

$$
\mathrm{R}_{i}=\log _{2}\left(1+\frac{\left|\mathbf{u}_{i}^{*} \mathbf{H}_{i 1} \mathbf{v}_{i}\right|^{2}}{\sigma^{2}+\left|\mathbf{u}_{i}^{*} \sum_{l=1, l \neq i}^{2} \mathbf{H}_{l 1} \mathbf{v}_{l}\right|^{2}+\left|\mathbf{u}_{i}^{*} \sum_{j=3}^{4} \mathbf{G}_{j 1} \mathbf{v}_{j}\right|^{2}}\right) .
$$

Ergodic mean sum rate can be found as

$$
\tilde{\mathrm{R}}=\sum_{i=1}^{4} \mathbb{E}\left\{R_{i}\right\}
$$

\subsubsection{Interference Alignment with Limited Feedback}

Authors of [100] and [101] considered limited feedback. They applied RVQ in [103]. BSs exchange the adjacent cell precoding vectors. Each BS then quantizes the precoding vectors for the served users by employing a quantization 
codebook, $\mathcal{C}=\left\{\mathbf{c}_{1}, \mathbf{c}_{2}, \ldots \mathbf{c}_{Q}\right\}$. Each codebook consists of $Q$-dimensional unit norm vectors of size $Q=2^{B}$, where $B$ is the number of bits for feedback channel. Indices for the codebooks, which will be used as precoding vectors, are found using the minimum chordal distance metric as

$$
\hat{\mathbf{v}}_{i}=\mathbf{c}_{k_{j}}, \quad k_{j}=\arg \max _{1 \leq m \leq 2^{B}}\left|\mathbf{c}_{m} \mathbf{v}_{i}\right| .
$$

These codebooks are known at transmitters as well. After quantization, each BS informs indices $k_{j}$ to its served user through the limited feedback channel $B$ bps rates. In [100], the authors also proposed to scale how many feedback bits $B$ to be used according to SNR as

$$
B \geq\left(N_{r}-1\right) \log _{2}(\rho)+C
$$

where $\rho$ is the transmit SNR and $C$ is a constant which is defined as $C=$ $(M-1) \log _{2}\left(\frac{\sum_{j=1, j \leq i} A_{j}}{\tau-1}\right), i$ is the desired user index, $j$ is the interfering user index and $A_{j}$ denotes the expectation of the effective interference channel. In [100], the exact distribution is not given. However, it is given that $A_{j} \approx 1.5 . \tau$ is also chosen as 2 in [100].

In [100] the achievable rate for a limited feedback scheme above is given as

$$
\hat{R}_{i}=\log _{2}\left(1+\frac{\left|\mathbf{u}_{i}^{*} \mathbf{H}_{i 1} \hat{\mathbf{v}}_{i}\right|^{2}}{\sigma^{2}+\left|\mathbf{u}_{i}^{*} \sum_{l=1, l \neq i}^{2} \mathbf{H}_{l 1} \hat{\mathbf{v}}_{l}\right|^{2}+\left|\mathbf{u}_{i}^{*} \sum_{j=3}^{4} \mathbf{G}_{j 1} \hat{\mathbf{v}}_{j}\right|^{2}}\right) .
$$

In (4.20), one could note that the postcoders are used as same as given in (4.12 - 4.15). However, in [101], it is shown that the rate loss can be eliminated if the postcoders are updated with codebooks as given by

$$
\begin{aligned}
& \hat{\mathbf{u}}_{1}^{*}\left[\begin{array}{ll}
\mathbf{H}_{21} \hat{\mathbf{v}}_{2} & \boldsymbol{\eta}_{1}^{\mathrm{ICI}}
\end{array}\right]=0 \\
& \hat{\mathbf{u}}_{2}^{*}\left[\begin{array}{ll}
\mathbf{H}_{11} \hat{\mathbf{v}}_{1} & \boldsymbol{\eta}_{\mathbf{1}}^{\mathrm{ICI}}
\end{array}\right]=0 .
\end{aligned}
$$

Similarly, the BS 2 can update the postcoders

$$
\begin{aligned}
& \hat{\mathbf{u}}_{3}^{*}\left[\begin{array}{ll}
\mathbf{H}_{42} \hat{\mathbf{v}}_{4} & \boldsymbol{\eta}_{\mathbf{2}}^{\mathrm{ICI}}
\end{array}\right]=0 \\
& \hat{\mathbf{u}}_{4}^{*}\left[\begin{array}{ll}
\mathbf{H}_{32} \hat{\mathbf{v}}_{3} & \boldsymbol{\eta}_{\mathbf{2}}^{\mathrm{ICI}}
\end{array}\right]=0 .
\end{aligned}
$$

Because the codebooks are decided at the receiver, the receiver can update the postcoding vector with the chosen codebook as a precoding vector. Thus, the mean sum rates can be improved [101]. 


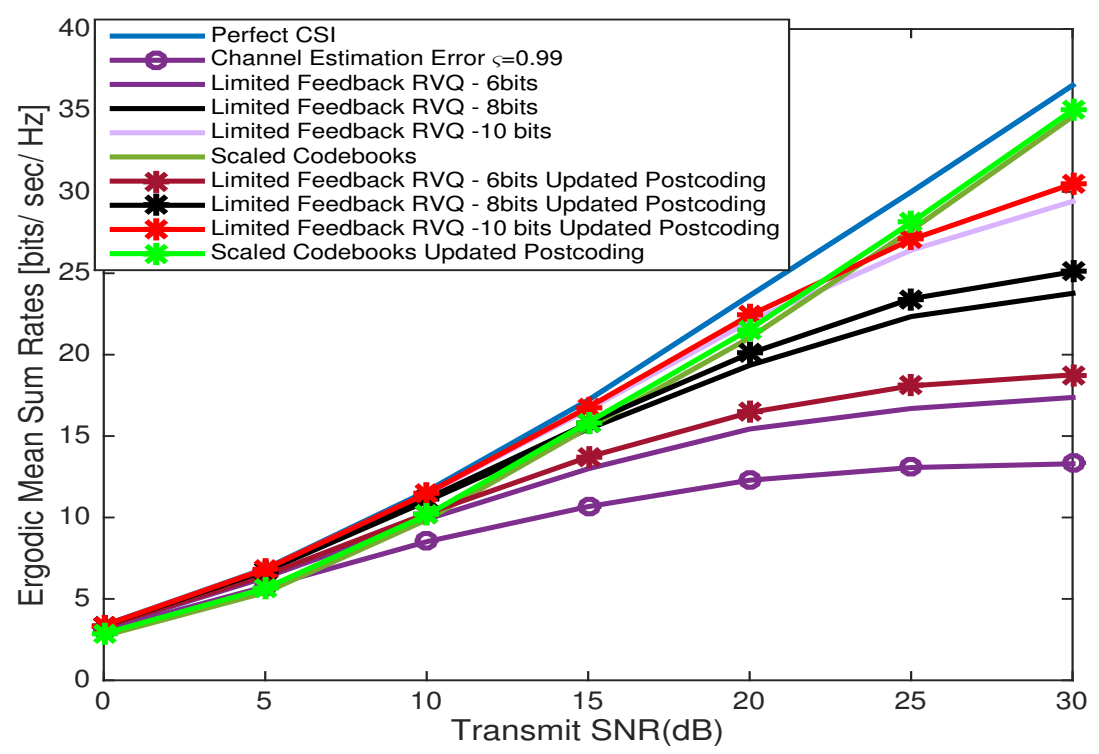

Figure 4.2: Ergodic mean sum rates for 2 - cell uplink network with imperfect CSI and limited feedback

\subsubsection{Simulation Results}

In this section we reproduce the results of [100] and [101] and also add our results using the imperfect CSI model given in (2.23). We also use IEEE 802.16e codebooks mentioned in Chapter 2 for comparing with the RVQ codebooks of [100] and [101]. We also compare the ergodic mean sum rates in (4.17) by using codebooks and using the imperfect CSI model of (2.23).

In Fig. 4.2, mean sum rates are given considering different cases. As seen in the results, imperfect CSI and limited feedback deteriorate the mean sum rates significantly relative to the case of perfect CSI. However, increasing number of feedback bits improves the mean sum rates with limited feedback. We use the RVQ codebooks as mentioned earlier. It is also shown in the results that the scaled codebooks of [100] which are given in (4.19) provide better mean sum rates compared to the case using fixed number of bits for all transmit SNRs. We have also shown that updated postcoders of [101] provide better mean sum rates than using the postcoders of [100] as they achieve better cancellation at the receivers. As shown in Fig. 4.2, we can achieve sum rates with limited feedback, close to sum rates with perfect CSI. However, the number of bits used for codebooks have to be considerably large which is actually not practical.

In Fig. 4.3, we give mean sum rates using a 3 bits RVQ codebook. Here we also use the 3 bits codebook of IEEE 802.16e [104] and the imperfect CSI model in (2.23). Simulation results show that IEEE 802.16e codebooks provide better sum rates compared to RVQ. However, the loss in sum rates is quite big compared to perfect CSI as we used only 3 bits codebooks. In this case, the 


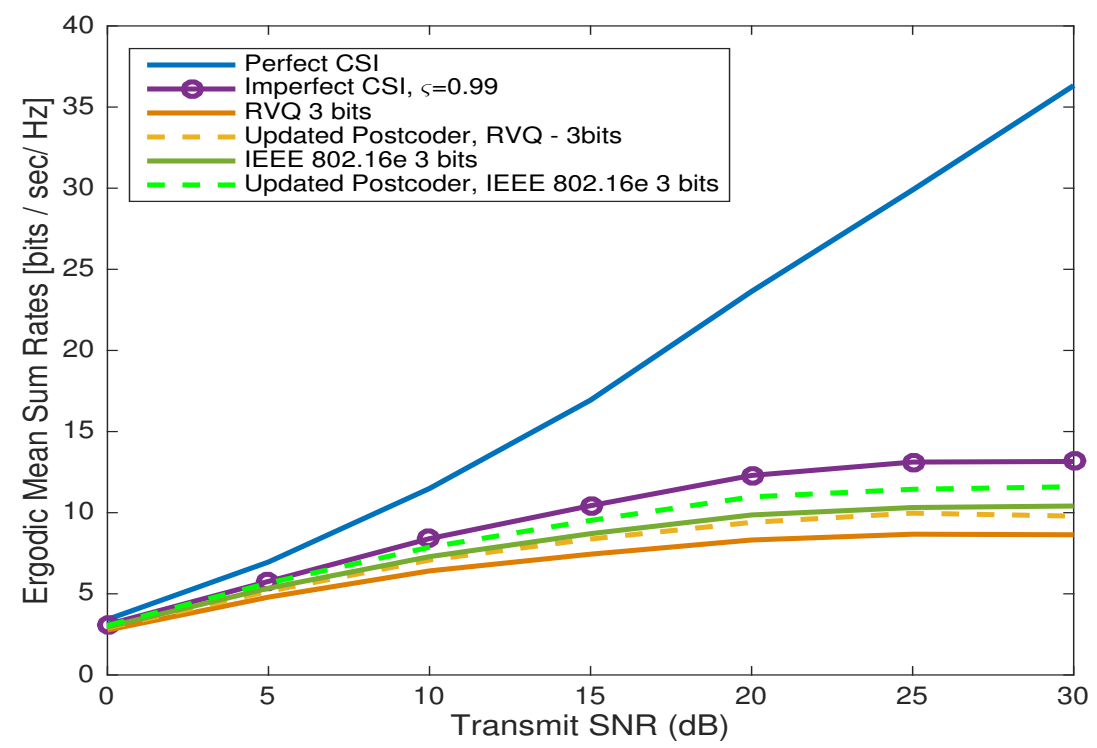

Figure 4.3: Ergodic mean sum rates for 2 - cell uplink network with imperfect CSI and limited feedback (3 bits only)

imperfect CSI model with $\varsigma=0.99$ provides better mean sum rates relative to the case of limited feedback.

\subsection{Interference Alignment for Multicell Networks in the Uplink}

In this section, we first discuss an IA scheme for a heterogeneous cellular network that we proposed in [1]. This can also be expanded to a multicellular macro-cell system. IA for multicellular systems is more complex as more dimensions are needed to align all interference in the same subspace. We address this problem by classifying the interfering sources as dominant and remaining. Dominant interferers are simply the interfering sources which have relatively stronger power, while remaining interferers have less power leaking to the destination.

\subsubsection{Interference Alignment with Combined Receivers For Heterogeneous Cellular Networks}

As mentioned earlier, ZF receivers can effectively cancel the interference, provided enough dimensions exist. However, the complete nulling of many dominant interferers with ZF receivers in a multi-user, multicellular case requires a large number of receive antennas. This becomes a challenge when we have a bigger network and many interfering sources. Here, we propose a novel combined receiver of [1] which applies ZF to null the dominant interferers followed by 
MMSE processing to reduce the effect of the remaining interference and noise. The receiver is used in conjunction with an IA scheme in a multicell environment. Simulation results considering a range of interferer profiles show that the combined receiver outperforms an MMSE receiver by ergodic mean sum rates, due to its ability to eliminate the dominant interferers. If the antennas are closely located in a compact array, then spatial correlation [105] is important and its impact on the IA scheme is also considered.

The idea of the combined receivers is to null out the dominant interferers via ZF, and subsequently reduce the remaining interference by the MMSE technique.

Our contributions in this subsection are as follows

- We consider an IA scheme with a novel combined receiver which nulls the dominant interferers with ZF and subsequently uses MMSE processing to reduce the effects of the remaining interference and noise.

- We implement our scheme in an IA heterogeneous uplink network which involves a mix of macro and pico cells.

- We show the range of remaining to dominant interference ratios in which our receiver outperforms the traditional MMSE receiver.

The concept of IA for heterogeneous cellular uplink networks [106] is given in Fig. 4.4.

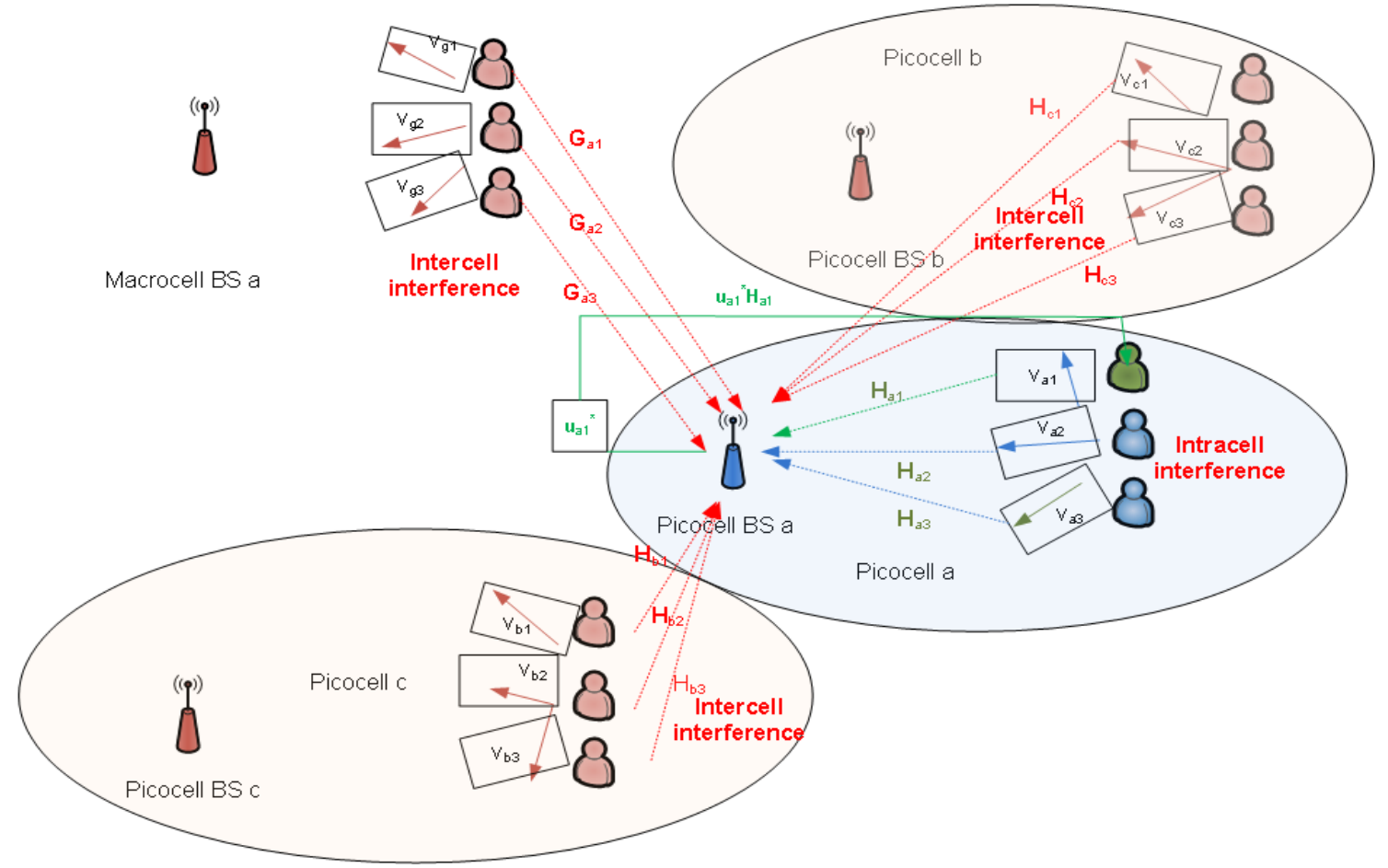

Figure 4.4: System model of the uplink heterogeneous cellular network of [1] 
Consider the BS of Picocell $a$ where interference will be aligned and mitigated. The received signal at this $\mathrm{BS}$ can be written as

$$
\begin{aligned}
\mathbf{y}_{a} & =\sum_{i=1}^{K}\left(\sqrt{\tilde{\rho}_{a, i}} \mathbf{H}_{a, i} \mathbf{v}_{a, i} x_{a, i}+\sqrt{\tilde{\rho}_{b, i}} \mathbf{H}_{b, i} \mathbf{v}_{b, i} x_{b, i}\right. \\
& \left.+\sqrt{\tilde{\rho}_{c, i}} \mathbf{H}_{c, i} \mathbf{v}_{c, i} x_{c, i}+\sqrt{\tilde{\rho}_{g, i}} \mathbf{G}_{a, i} \mathbf{v}_{g, i} x_{g, i}\right)+\mathbf{n}_{a},
\end{aligned}
$$

where $K$ is the number of users in each cell, $\mathbf{n}_{a}$ is additive white Gaussian noise (AWGN) vector which is normalised to variance 1 and $\tilde{\rho}_{a, i}, \tilde{\rho}_{b, i}, \tilde{\rho}_{c, i}$ and $\tilde{\rho}_{g, i}$ are the mean powers ${ }^{1}$ of the channels $\mathbf{H}_{a, i}, \mathbf{H}_{b, i}, \mathbf{H}_{c, i}, \mathbf{G}_{a, i}$ respectively, where

- $\mathbf{H}_{a, i}$ : The channel of the $i$ th user in Picocell a to its own BS.

- $\mathbf{H}_{b, i}, \mathbf{H}_{c, i}, \mathbf{G}_{a, i}$ : The channels of the $i$ th interfering user in picocell $b, c$ and macrocell to the BS of picocell $a$ respectively.

Ideally, channels are assumed to be perfectly known at both transmitters and receivers. However, applying some channel estimation errors will naturally result in performance degradation. The users transmit their precoded data, $x$, using the vectors $\mathbf{v}$, as shown in Fig 4.4, where the subscripts denote the originating cell. For concreteness, we consider 3 picocells and one macrocell with equal number of users. However, this can be generalised to any system dimension as required. The BS uses a postcoding vector $\mathbf{u}_{a, l}^{\mathrm{CR}}$ to decode the $l$ th user's signal as

$$
\begin{aligned}
\tilde{x}_{a, l} & =\mathbf{u}_{a, l}^{\mathrm{CR} *} \mathbf{y}_{a} \\
& =\mathbf{u}_{a, l}^{\mathrm{CR} *}(\underbrace{\sqrt{\tilde{\rho}_{a, l}} \mathbf{H}_{a, l} \mathbf{v}_{a, l} x_{a, l}}_{\text {Desired Signal }}+\underbrace{\sum_{i=1, i \neq l}^{K}\left(\sqrt{\tilde{\rho}_{a, i}} \mathbf{H}_{a, i} \mathbf{v}_{a, i} x_{a, i}\right)}_{\text {Intracell Interference }} \\
& +\underbrace{\sum_{i=1}^{K}\left(\sqrt{\tilde{\rho}_{b, i}} \mathbf{H}_{b, i} \mathbf{v}_{b, i} x_{b, i}+\sqrt{\tilde{\rho}_{c, i}} \mathbf{H}_{c, i} \mathbf{v}_{c, i} x_{c, i}\right.}_{\text {Intercell Interference }} \\
& +\underbrace{\left.\sqrt{\tilde{\rho}_{g, i}} \mathbf{G}_{a, i} \mathbf{v}_{g, i} x_{g, i}\right)}_{\text {Intercell Interference }}+\underbrace{\mathbf{n}_{a}}_{\text {Noise }}),
\end{aligned}
$$

where the superscript CR denotes the combined receiver. Considering (4.26), the BS cancels the interference coming from out-of-cell users (intercell interference) by application of the combined receiver. The interference coming from the users in the same cell (intracell interference) is aligned by application of precoders, $\mathbf{v}$, at the transmitters.

\footnotetext{
${ }^{1}$ Once the location of the picocells is fixed, the random user locations within the macrocell and picocell would then in practice determine the appropriate mean power levels for the dominant and the remaining interferers.
} 


\subsubsection{Scenarios Considered for Heterogeneous Networks}

We consider 3 scenarios to evaluate the performance of the proposed combined receiver. In all three scenarios, we consider 3 users in each cell. Each user is equipped with $N_{t}$ antennas, while each BS has $N_{r}$ antennas. We assume there are some dominant interferers with mean total power, $\tilde{\rho}_{\text {dom }}$, while the remaining interferers are weaker with mean total power $\tilde{\rho}_{\text {rem }}$. Similar to [3], we parametrise the system using

$$
\tilde{\gamma}=\frac{\tilde{\rho}_{\text {rem }}}{\tilde{\rho}_{\text {dom }}}
$$

In Scenario 1, the BS of picocell $a$ nulls out only 1 interfering macrocell user (assumed dominant) via ZF while MMSE processing reduces the effects of interference caused by the remaining 2 macrocell users and other interfering users in picocell $b$ and picocell $c$. The mean power of the channel of the dominant macrocell user (say user 1) is $\tilde{\rho}_{\text {dom }}=\tilde{\rho}_{g, 1}=\frac{\text { SNR }}{N_{t}}$ where SNR is the Signal to Noise Ratio. Since we have normalised the noise variance to $1, \mathrm{SNR}$ is equivalent to the power. The mean channel powers of users in picocells $b$ and $c$ are assumed to be equal to each other $\tilde{\rho}_{b, i}=\tilde{\rho}_{c, i}=\psi_{1} \tilde{\rho}_{g, 1}, \quad(i=1, \ldots, K)$. The mean channel power for the remaining two users in the macrocell is given as $\tilde{\rho}_{g, 2}=\psi_{2} \tilde{\rho}_{g, 1}$ and $\tilde{\rho}_{g, 3}=\psi_{3} \tilde{\rho}_{g, 1}$ respectively. We assume the coefficients $0<\psi_{1}, \psi_{2}, \psi_{3}<1$ as all these interferers are assumed weaker. The mean total power for remaining interference is then $\tilde{\rho}_{\text {rem }}=\sum_{i=1}^{K}\left(\tilde{\rho}_{b, i}+\tilde{\rho}_{c, i}\right)+\tilde{\rho}_{g, 2}+\tilde{\rho}_{g, 3}$.

Scenarios $\mathbf{2}$ and $\mathbf{3}$ are the most aggressive and are considered to evaluate the gains of the combined receiver. Here, we assume that the interfering users' powers are much stronger than in Scenario 1. In Scenarios 2 and 3 we assume that all 3 users in macrocell $a$ and picocell $b$ are dominant and the mean powers of their channels are equal to the mean power of the desired user's channel $\left(\tilde{\rho}_{a, i}=\tilde{\rho}_{g, i}=\tilde{\rho}_{b, i}=\frac{\mathrm{SNR}}{N_{t}}\right)$. The interference caused by the users of picocell $c$ is considered as weak remaining interference. All users of this picocell have the same mean power, $\tilde{\rho}_{c, i}=\tilde{\gamma}\left(\tilde{\rho}_{g, i}+\tilde{\rho}_{b, i}\right), \quad(i=1, \ldots, K)$. The total mean powers for dominant and remaining interference are then written as $\tilde{\rho}_{\text {dom }}=$ $\sum_{i=1}^{K}\left(\tilde{\rho}_{g, i}+\tilde{\rho}_{b, i}\right)$ and $\tilde{\rho}_{\text {rem }}=\sum_{i=1}^{K}\left(\tilde{\rho}_{c, i}\right)$, respectively.

In Scenario 2, we assume that the BS uses ZF to null out all of the dominant user interference while applying MMSE to the remaining interference.

In Scenario 3, the BS nulls out the interference from only 2 users in each dominant interfering cell. The rest of the interference is handled by MMSE processing.

As discussed earlier Scenario 2 requires more antennas at the receiver than Scenario 3 because of the number of interfering sources to be ZF'd. 


\subsubsection{Combined Receivers}

In this section, we explain the design of the combined ZF/MMSE receiver. For simplicity, only Scenarios 2 and 3 are described below as these are the most complex. The simplification of the equations for Scenario 1 is trivial and modification of equations for Scenario 1 is provided following Scenario 2 and 3. In order to reduce the notational complexity, we drop the mean power of the channels in the equations, as all interference levels are equal for both scenarios. Our aim is to null the dominant interferers from picocell $b$ and the macrocell by the use of ZF and reduce the remaining interference plus noise with MMSE. Remaining interference changes depending on the scenario. We denote the postcoding vector at the BS for the $l$ th user's signal as

$$
\mathbf{u}_{a, l}^{\mathrm{CR}}=\frac{\tilde{\mathbf{U}}_{a, l} \mathbf{w}_{a, l}}{\left\|\tilde{\mathbf{U}}_{a, l} \mathbf{w}_{a, l}\right\|},
$$

where $\tilde{\mathbf{U}}_{a, l}$ and $\mathbf{w}_{a, l}$ are the ZF part and the MMSE part of the receiver respectively.

- ZF Part of the Receiver We first take the singular value decomposition of the dominant interference channel matrices for picocell $a$. This gives

$$
\begin{aligned}
\boldsymbol{\Psi}_{a, l} & =\underbrace{\left[\begin{array}{lll}
\mathbf{H}_{b 1} \ldots \mathbf{H}_{b K_{l}} \mathbf{G}_{a 1} \ldots \mathbf{G}_{a K_{l}}
\end{array}\right]}_{N r \times 2 K_{l} N_{t}} \\
& =\underbrace{\left[\mathbf{U}_{a, l}\right.}_{N_{r} \times N_{r}}] \underbrace{\left[\mathbf{D}_{a, l}\right.}_{N_{r} \times 2 K_{l} N_{t}}] \quad \underbrace{\left[\mathbf{V}_{a, l}^{*}\right.}_{2 K_{l} N_{t} \times 2 K_{l} N_{t}}]
\end{aligned},
$$

where $\boldsymbol{\Psi}_{a, l}$ is the concatenation of the dominant intercell interference channels for the user $l$ in picocell $a$. We assume that $N_{t}$ and $N_{r}$ are the same for all sources and receivers for simplicity. $K_{l}$ is the number of dominant interfering users from each cell to be nulled out with ZF. In (4.29) the matrix, $\mathbf{D}_{a, l}$, is defined as

$$
\mathbf{D}_{a, l}=\left[\begin{array}{ccc}
\sqrt{\lambda_{1}} & 0 & \ldots \\
\vdots & \ddots & \vdots \\
0 & \cdots & \sqrt{\lambda_{2 K_{l} N_{t}}} \\
0 & \cdots & 0 \\
\vdots & \ddots & \vdots \\
0 & \cdots & 0
\end{array}\right]
$$

Here the last $N_{r}-2 K_{l} N_{t}$ rows of $\mathbf{D}_{a, l}$ are zeros. This should not be less than the number of interfering streams to be nulled with ZF which is $2 K_{l} N_{t}$. Therefore $N_{r} \geq 4 K_{l} N_{t}$. Using this, $N_{r} \geq 12 N_{t}$ and $N_{r} \geq 8 N_{t}$ for 
Scenarios 2 and 3 respectively.

We can denote $\mathbf{U}_{a, l}$ as

$$
\mathbf{U}_{a, l}=[\underbrace{\overline{\mathbf{U}}_{a, l}}_{N_{r} \times 2 K_{l} N_{t}} \mid \underbrace{\tilde{\mathbf{U}}_{a, l}}_{N_{r} \times\left(N_{r}-2 K_{l} N_{t}\right)}],
$$

where $\tilde{\mathbf{U}}_{a, l}$ is the ZF part of the receiver.

- MMSE Part of the Receiver The MMSE Part of the receiver denoted as $\mathbf{w}_{a, l}$ in (4.28) aims to reduce the remaining interference from the other picocells and also the AWGN.

The equivalent desired channels can be written as

$$
\tilde{\mathbf{H}}_{a, l}=\tilde{\mathbf{U}}_{a, l}^{*}\left(\sqrt{\tilde{\rho}_{a, i}} \mathbf{H}_{a, l} \mathbf{v}_{a, l}\right),
$$

where $\mathbf{v}_{a, l}$ is the precoding vector. Similarly, we can write the equivalent interference channels from picocells $b, c$ and the macrocell as

$$
\begin{aligned}
\tilde{\mathbf{H}}_{b, i} & =\tilde{\mathbf{U}}_{a, l}^{*}\left(\sqrt{\tilde{\rho}_{b, i}} \mathbf{H}_{b, i} \mathbf{v}_{b, i}\right), \\
\tilde{\mathbf{H}}_{c, i} & =\tilde{\mathbf{U}}_{a, l}^{*}\left(\sqrt{\tilde{\rho}_{c, i}} \mathbf{H}_{c, i} \mathbf{v}_{c, i}\right), \\
\tilde{\mathbf{G}}_{a, i} & =\tilde{\mathbf{U}}_{a, l}^{*}\left(\sqrt{\tilde{\rho}_{g, i}} \mathbf{G}_{a, i} \mathbf{v}_{g, i}\right) .
\end{aligned}
$$

Finally, we have the MMSE stage achieved by

$$
\mathbf{w}_{a, l}=(\underbrace{\tilde{\mathbf{H}}_{a, l} \tilde{\mathbf{H}}_{a, l}^{*}}_{\text {Desired }}+\boldsymbol{\Omega}+\underbrace{\mathbf{I}}_{\text {Noise }})^{-1} \tilde{\mathbf{H}}_{a, l},
$$

where $\Omega$ is the interference to be reduced by the MMSE processing, given as

$$
\begin{aligned}
\boldsymbol{\Omega} & =\sum_{i=1}^{K} \tilde{\mathbf{H}}_{c, i} \tilde{\mathbf{H}}_{c, i}^{*}+\sum_{i=K_{l}+1}^{K} \tilde{\mathbf{H}}_{b, i} \tilde{\mathbf{H}}_{b, i}^{*} \\
& +\sum_{i=K_{l}+1}^{K} \tilde{\mathbf{G}}_{a, i} \tilde{\mathbf{G}}_{a, i}^{*} .
\end{aligned}
$$

For Scenario 2, all picocell $b$ and macrocell users are nulled out with the ZF part $\left(K_{l}=K\right),(4.37)$ can be rewritten for Scenario 2 as $\boldsymbol{\Omega}=$ $\sum_{i=1}^{K} \tilde{\mathbf{H}}_{c, i} \tilde{\mathbf{H}}_{c, i}^{*}$.

\section{Modification of the equations for Scenario 1}

The simplification of post-processing for Scenario 1 would consist of using only the dominant macrocell user matrix $\mathbf{G}_{a, 1}$ in (4.29) and then appropriately mod- 
ifying (4.37) to include all the remaining interference. Note that as there are $N_{t}$ interfering streams to be nulled by the use of ZF, $N_{r}$ is equal to $2 N_{t}$ for Scenario 1. We can rewrite (4.29) for Scenario 1

$$
\begin{aligned}
\boldsymbol{\Psi}_{a, l} & =\underbrace{\left[\mathbf{G}_{a 1}\right]}_{N r \times N_{t}}] \\
& =\underbrace{\left[\mathbf{U}_{a, l}\right]}_{N_{r} \times N_{r}} \underbrace{\left[\mathbf{D}_{a, l}\right]}_{N_{r} \times N_{t}} \underbrace{\left[\mathbf{V}_{a, l}^{*}\right]}_{N_{t} \times N_{t}},
\end{aligned}
$$

Then we can rewrite $\mathbf{D}_{a, l}$ for Scenario 1

$$
\mathbf{D}_{a, l}=\left[\begin{array}{ccc}
\sqrt{\lambda_{1}} & 0 & \cdots \\
\vdots & \ddots & \vdots \\
0 & \cdots & \sqrt{\lambda_{N_{t}}} \\
0 & \cdots & 0 \\
\vdots & \ddots & \vdots \\
0 & \cdots & 0
\end{array}\right]
$$

Here the last $N_{r}-N_{t}$ rows of $\mathbf{D}_{a, l}$ are zeros. This should not be less than the number of interfering streams to be nulled with ZF which is $N_{t}$ for Scenario 1. Therefore $N_{r} \geq 2 N_{t}$.

For Scenario 1, the autocorrelation matrix of total interference to be reduced with the MMSE part, $\boldsymbol{\Omega}$ in (4.37), can be given by

$$
\boldsymbol{\Omega}=\sum_{i=1}^{K} \tilde{\mathbf{H}}_{c, i} \tilde{\mathbf{H}}_{c, i}^{*}+\sum_{i=1}^{K} \tilde{\mathbf{H}}_{b, i} \tilde{\mathbf{H}}_{b, i}^{*}+\sum_{i=2}^{K} \tilde{\mathbf{G}}_{a, i} \tilde{\mathbf{G}}_{a, i}^{*} .
$$

The rest of the equations are same for all scenarios.

\subsubsection{Proposed IA Algorithm of the Combined Receiver}

We now describe the proposed IA scheme.

- Initialization: The BS initialises its postcoding vector for the $i$ th user in Picocell $a$ as

$$
\mathbf{u}_{a, l}^{\mathrm{CR}(0)}=\tilde{\mathbf{U}}_{a, l} \mathbf{w}_{a, l}^{(0)}
$$

and

$$
\mathbf{w}_{a, l}^{(0)}=\left(\tilde{\mathbf{H}}_{a, l}^{(0)} \tilde{\mathbf{H}}_{a, l}^{(0) *}+\boldsymbol{\Omega}+\mathbf{I}\right)^{-1} \tilde{\mathbf{H}}_{a, l}^{(0)}
$$


where

$$
\tilde{\mathbf{H}}_{a, l}^{(0)}=\tilde{\mathbf{U}}_{a, l}^{*}\left(\sqrt{\tilde{\rho}_{a, l}} \mathbf{H}_{a, l} \mathbf{v}_{a, l}^{(0)}\right)
$$

In (4.43), we set $\mathbf{v}_{a, l}^{(0)}$ as the maximum eigenvector of $\mathbf{H}_{a, l}^{*} \mathbf{H}_{a, l}$. The BS then feeds back the equivalent channel $\mathbf{u}_{a, l}^{\mathrm{CR}(0) *} \mathbf{H}_{a, l}$ to the user $a$.

- Designing Precoding Vectors: The precoding vectors are generated to align the intercell interference at the BS. These should satisfy the following conditions

$$
\begin{aligned}
& \tilde{\mathbf{v}}_{a, 1}^{(1)} \subset \operatorname{null}\left(\left[\mathbf{u}_{a, 2}^{\mathrm{CR}(0) *} \mathbf{H}_{a, 1} ; \mathbf{u}_{a, 3}^{\mathrm{CR} *} \mathbf{H}_{a, 1}\right]\right), \\
& \tilde{\mathbf{v}}_{a, 2}^{(1)} \subset \operatorname{null}\left(\left[\mathbf{u}_{a, 1}^{\mathrm{CR}(0) *} \mathbf{H}_{a, 2} ; \mathbf{u}_{a, 3}^{\mathrm{CR} *} \mathbf{H}_{a, 2}\right]\right), \\
& \tilde{\mathbf{v}}_{a, 3}^{(1)} \subset \operatorname{null}\left(\left[\mathbf{u}_{a, 1}^{\mathrm{CR}(0) *} \mathbf{H}_{a, 3} ; \mathbf{u}_{a, 2}^{\mathrm{CR} *} \mathbf{H}_{a, 3}\right]\right),
\end{aligned}
$$

where null(A) denotes an orthonormal bases for the null space of the matrix A. These precoding vectors should be selected from the subset of vectors spanning the null space. The size of the null space is dependent on the number of dimensions. Then these precoding vectors are normalised as

$$
\mathbf{v}_{a, i}^{(1)}=\frac{\tilde{\mathbf{v}}_{a, i}^{(1)}}{\left\|\tilde{\mathbf{v}}_{a, i}^{(1)}\right\|}
$$

Note that $\Omega$ in (4.42) does not change with iteration, because we assume that the BSs do not have information of updated out-of-cell precoding vectors as in [3]. However, similar to (4.44), the adjacent BSs also update their precoding vectors to null intracell interference. Therefore, the complete nulling of intercell interference with MMSE receivers is not possible due to unknown out-of-cell precoding vectors. In [3], the authors proposed to use the expected value of the out-of-cell precoding vectors. Therefore, when $\boldsymbol{\Omega}$ in (4.42) is calculated, we use $\mathbb{E}\left[\mathbf{v}_{b, i} \mathbf{v}_{b, i}^{*}\right]=\mathbb{E}\left[\mathbf{v}_{c, i} \mathbf{v}_{c, i}^{*}\right]=\mathbb{E}\left[\mathbf{v}_{g, i} \mathbf{v}_{g, i}^{*}\right]=\frac{1}{N t} \mathbf{I}$ which is found via simulations. These findings are also supported by the analysis of $[107,108]$.

- Iteration: In this step, iteration and scheduling, i.e. selecting users to maximise sum rates, may also be applied as in [3]. However, in this section, we do not consider any scheduling. Then the selected users inform the BS of their precoding vectors $\mathbf{v}_{a, i}^{(1)}$ via precoded pilots. Each BS updates the postcoding vector as

$$
\mathbf{u}_{a, l}^{\mathrm{CR}(1)}=\tilde{\mathbf{U}}_{a, l} \mathbf{w}_{a, l}^{(1)},
$$


where

$$
\mathbf{w}_{a, l}^{(1)}=\left(\tilde{\mathbf{H}}_{a, l}^{(1)} \tilde{\mathbf{H}}_{a, l}^{(1) *}+\boldsymbol{\Omega}+\mathbf{I}\right)^{-1} \tilde{\mathbf{H}}_{a, l}^{(1)}
$$

with

$$
\tilde{\mathbf{H}}_{a, l}^{(1)}=\tilde{\mathbf{U}}_{a, l}^{*} \mathbf{H}_{a, l} \mathbf{v}_{a, l}^{(1)} \text {. }
$$

\subsubsection{Performance Analysis for Heterogeneous Networks}

The performance of the proposed system is gauged by the following metrics.

The instantaneous SINR for the $l$ th user in picocell $a$ is defined by

$$
\operatorname{SINR}_{a, l}=\frac{\tilde{\rho}_{a, l}\left|\mathbf{u}_{a, l}^{\mathrm{CR} *} \mathbf{H}_{a, l} \mathbf{v}_{a, l} \mathbf{v}_{a, l}^{*} \mathbf{H}_{a, l}^{*} \mathbf{u}_{a, l}^{\mathrm{CR}}\right|}{1+\mathrm{INR}_{a, l}}
$$

where $\mathrm{INR}_{a, l}$ is the interference-to-noise ratio which can be written as

$$
\begin{aligned}
\mathrm{INR}_{a, l} & =\left|\mathbf{u}_{a, l}^{\mathrm{CR} *}\left(\sum_{i=1, i \neq l}^{K} \tilde{\rho}_{a, i} \mathbf{H}_{a, i} \mathbf{v}_{a, i} \mathbf{v}_{a, i}^{*} \mathbf{H}_{a, i}^{*}\right) \mathbf{u}_{a, l}^{\mathrm{CR}}\right| \\
& +\left|\mathbf{u}_{a, l}^{\mathrm{CR} *}\left(\sum_{i=1}^{K} \tilde{\rho}_{b, i} \mathbf{H}_{b, i} \mathbf{v}_{b, i} \mathbf{v}_{b, i}^{*} \mathbf{H}_{b, i}^{*}\right) \mathbf{u}_{a, l}^{\mathrm{CR}}\right| \\
& +\left|\mathbf{u}_{a, l}^{\mathrm{CR} *}\left(\sum_{i=1}^{K} \tilde{\rho}_{c, i} \mathbf{H}_{c, i} \mathbf{v}_{c, i} \mathbf{v}_{c, i}^{*} \mathbf{H}_{c, i}^{*}\right) \mathbf{u}_{a, l}^{\mathrm{CR}}\right| \\
& +\left|\mathbf{u}_{a, l}^{\mathrm{CR} *}\left(\sum_{i=1}^{K} \tilde{\rho}_{g, i} \mathbf{G}_{a, i} \mathbf{v}_{g, i} \mathbf{v}_{g, i}^{*} \mathbf{G}_{a, i}^{*}\right) \mathbf{u}_{a, l}^{\mathrm{CR}}\right| .
\end{aligned}
$$

The second metric is the ergodic mean sum rate for picocell $a$

$$
\tilde{\mathrm{R}}_{a}=\sum_{i=1}^{K} \mathbb{E}\left\{R_{a, i}\right\}
$$

where

$$
\mathrm{R}_{a, i}=\log _{2}\left(1+\mathrm{SINR}_{a, i}\right)
$$

\subsubsection{Simulation Results}

We evaluate the performance of the proposed IA scheme with combined receivers through simulations. We analyse the performance of the BS in picocell $a$ for all three scenarios described previously. We do not consider any iteration as it would increase the system overhead. 
In order to evaluate the scenarios, we need to determine the mean powers of the interferers. Once the location of the picocells is fixed, the random user locations within the macrocell and picocell would then in practice determine the appropriate mean power levels for the dominant and the remaining interferers. In [1], we have not considered random user locations and simply fix the values of the mean power levels of the interferers. This is because the purpose of this study is to evaluate and compare the IA scheme. Intracell interference is "nulled" via the null space precoding in (4.44-4.46). The null space precoding involves 3 users in each cell, therefore the minimum number of transmit antennas is $N_{t}=3$. The number of receive antennas, $N_{r}$, is scenario dependent. Note that scheduling is not considered in the simulations.

In Fig. 4.5, for Scenario 1, we present the ergodic mean sum rates as given by (4.53) of the proposed combined receiver IA approach and compare it with the MMSE based IA approach which is simply the approach given in [3] with a traditional MMSE receiver. We assume $\psi_{1}=0.04, \psi_{2}=0.01$ and $\psi_{3}=0.05$. Hence $\tilde{\gamma}=\frac{\left(\psi_{1}+\psi_{2}+\psi_{3}\right) \rho_{g, 1}}{\rho_{g, 1}}=0.1$. As in the previous discussion, $N_{r}=6$, (i.e. $2 N_{t}$ ) for this scenario. All channels are i.i.d complex Gaussian and the noise is AWGN. The results indicate that, even though we completely null out only 1 dominant

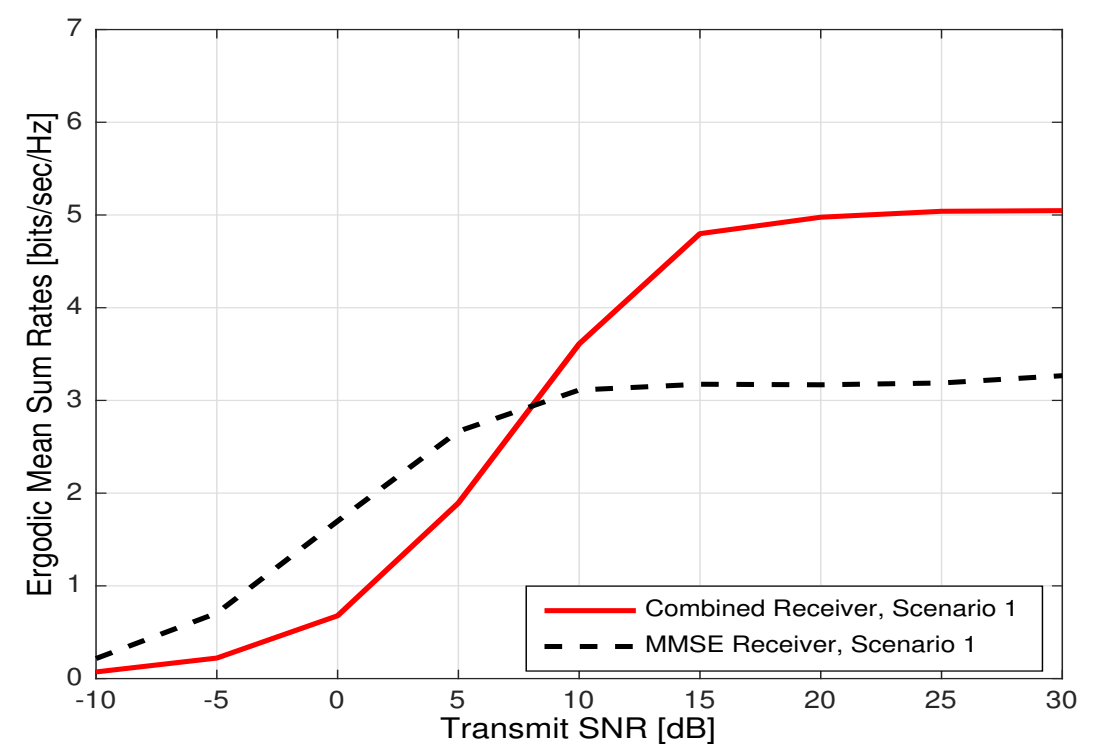

Figure 4.5: Ergodic mean sum rates for scenario 1 of uplink heterogeneous cellular networks, $N_{t}=3, N_{r}=6$

interferer with the ZF part, the proposed approach achieves better sum rates at higher SNR than the MMSE receiver. For low SNRs there are no dominant interferers, and ZF is not being utilised to its full potential. Therefore, because of the noise-enhancing feature of the ZF part of the combined receiver, the combined receiver performs poorer in the noise limited environments (low transmit SNRs). 
Next, we evaluate the simulations for Scenarios 2 and 3. In Fig. 4.6, we assume $N_{t}=3$ and $\tilde{\gamma}=0.2$ for both scenarios. Scenarios 2 and 3 have many dominant interferers. Both of these scenarios require a large number of antennas, therefore we consider the impact of antenna correlation as given in (2.25) in Chapter 2.

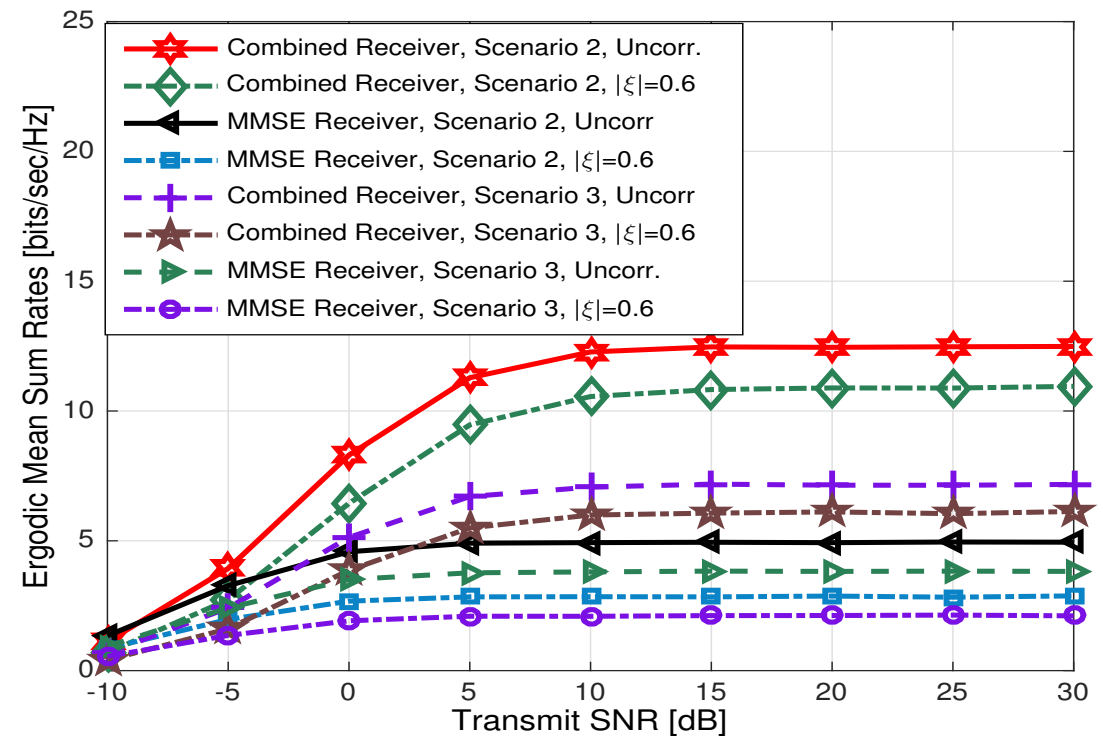

Figure 4.6: Ergodic mean sum rates of both scenario 2 and scenario 3 of uplink heterogeneous cellular networks for $N_{t}=3, \tilde{\gamma}=0.2$

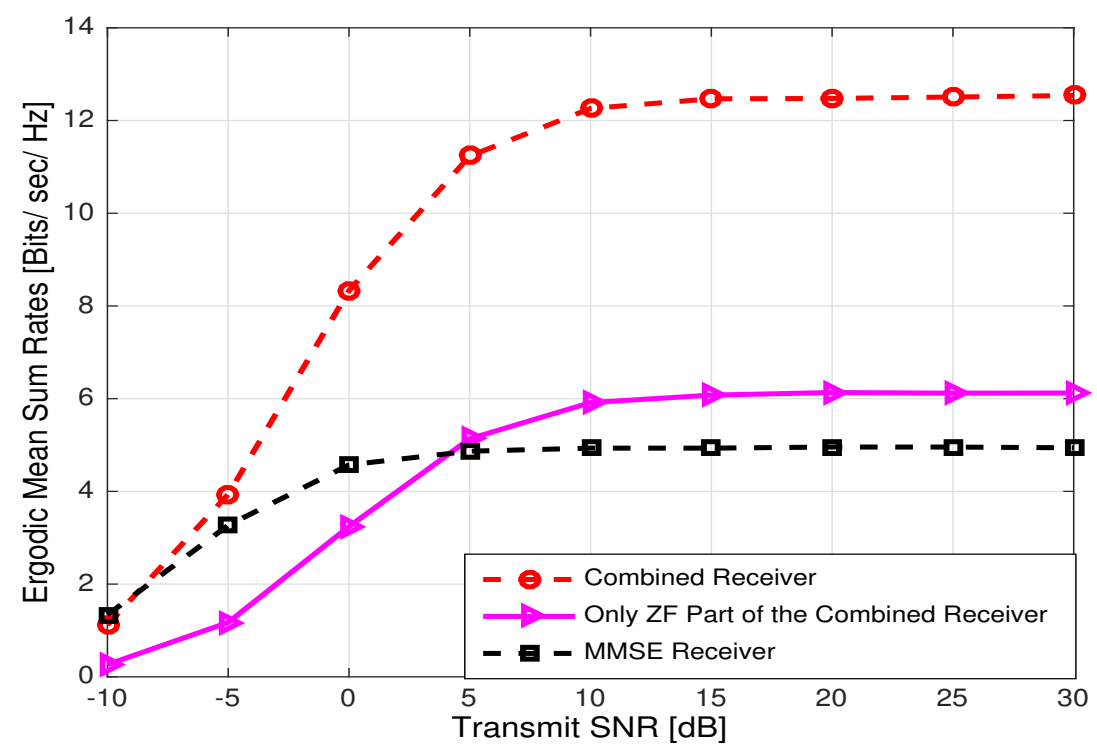

Figure 4.7: Ergodic mean sum rates of scenario 2 using combined receiver, Only $\mathrm{ZF}$ part of this combined receiver and MMSE receiver $N_{t}=3, \tilde{\gamma}=0.2$

The simulation results in Fig. 4.6 show that the combined receiver IA significantly outperforms the MMSE receiver IA. The gain in Scenario 3, where we 
null out fewer dominant interferers, is reduced as compared to Scenario 2. This is intuitively justifiable as some dominant interferers are being added to the remaining interferers and are mitigated via the MMSE part. The correlated results with $|\xi|=0.6$ result in a degradation in the performance. However, we see that the combined receiver still significantly outperforms the MMSE receiver. Antenna correlation impacts the MMSE receiver more significantly than the combined receiver, which is further explained in Fig. 4.9.

Considering the results given in Fig. 4.5 and Fig. 4.6, the transmit SNR region where the combined receiver becomes advantageous is higher for Scenario 1, compared to Scenario 2 and 3. This is because the interference levels are lower for Scenario 1 as mentioned in Section 4.2.2 and in the description of Fig. 4.5 and Fig. 4.6.

In Fig. 4.7, we investigate which part of the combined receiver is providing the gain. We show the ergodic mean sum rate for a ZF-only receiver where only the dominant interferers are nulled and the remaining interferers are ignored. We compare this with the combined receiver and the MMSE receiver for Scenario 2 .

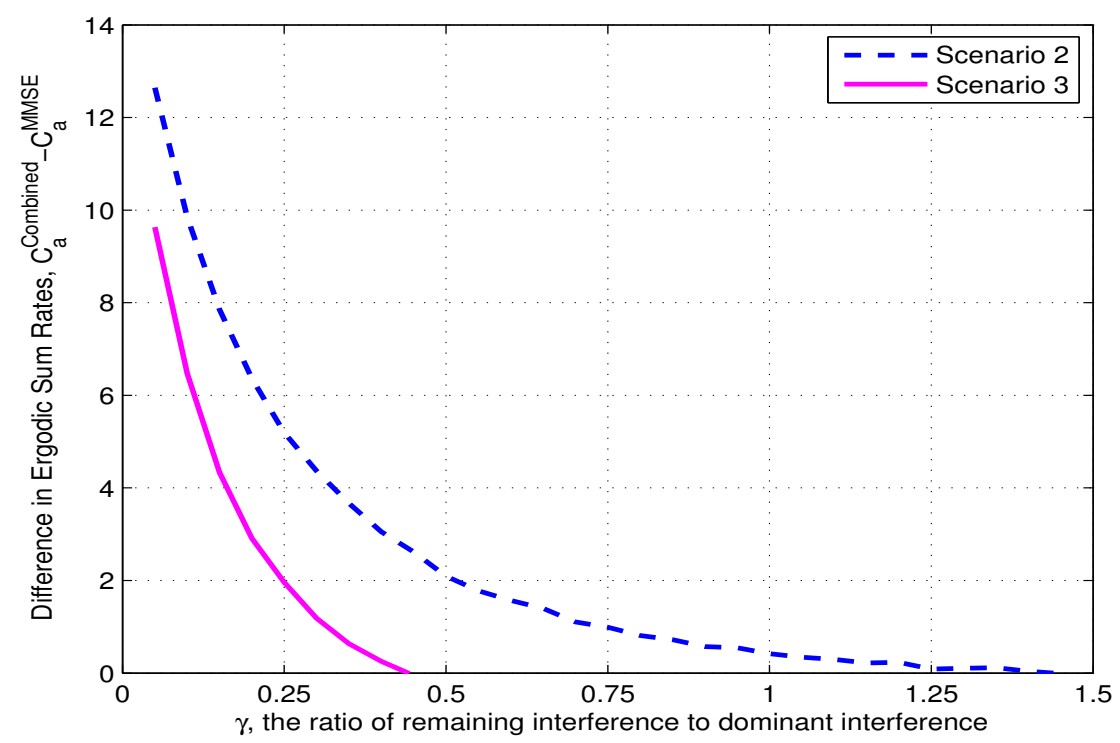

Figure 4.8: Difference between the sum rates of proposed combined receivers and MMSE receivers for various $\tilde{\gamma}$ ratios, $\mathrm{SNR}=5 \mathrm{~dB}, N_{t}=3$

Here we see that it can be beneficial to cancel dominant interferers as the ZF part results in a gain over the MMSE receiver for large SNRs. At lower SNRs, there is no dominant interferer, hence the MMSE receiver is superior. For high SNR, however, when both dominant and remaining interference are present, both receivers are required to obtain the large gains.

In Fig. 4.8, we give the difference between the sum rates of the combined receiver IA and the MMSE IA for different $\tilde{\gamma}$ values for both Scenario 2 and Scenario 
3. In doing so, we can analyse the range of $\tilde{\gamma}$ values where the performance of the combined receiver converges to that of the MMSE receiver. We evaluate the simulations for $\mathrm{SNR}=5 \mathrm{~dB}$. The results show that the combined receiver IA provides a huge gain when $\tilde{\gamma}$ is close to zero i.e. the remaining interference is very much weaker than the strong dominant nearby interfering cells. However, naturally this gain degrades as $\tilde{\gamma}$ increases. The simulation results also indicate that the combined receiver in Scenario 3 converges to MMSE much quicker than in Scenario 2, as it nulls out less interference with ZF in Scenario 3, relative to Scenario 2.

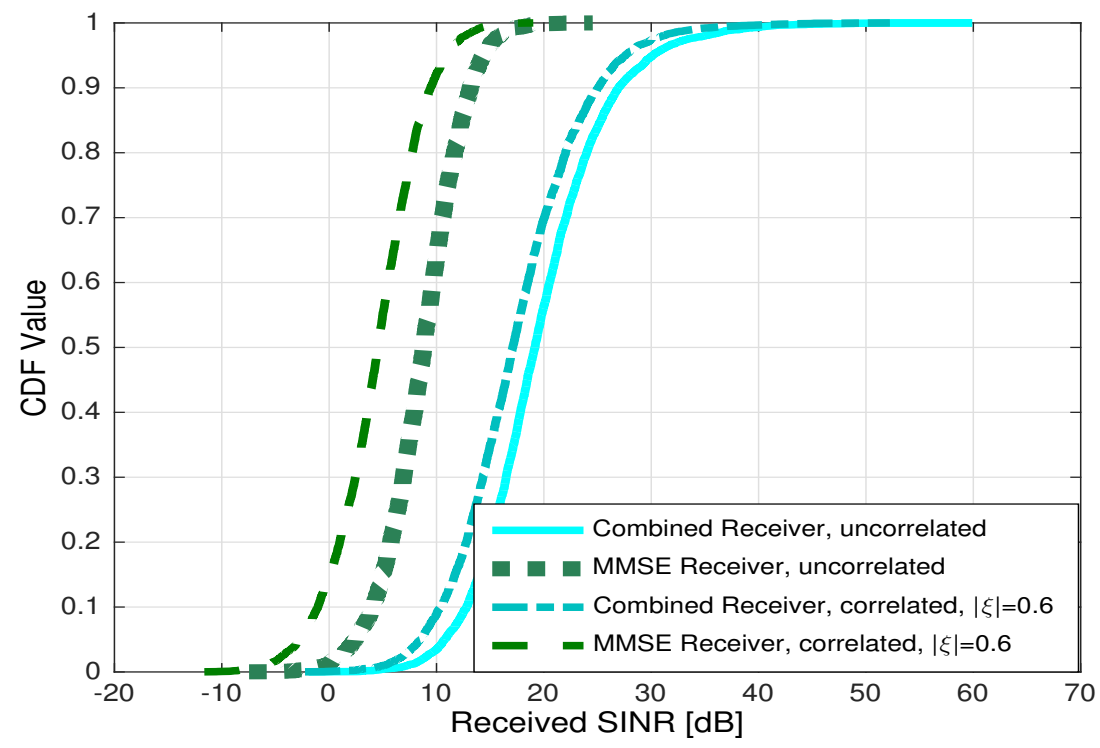

Figure 4.9: CDF of SINR, scenario 2 for correlated and uncorrelated channels, $\tilde{\gamma}=0.2, \quad N_{t}=3$

Finally, we show the cumulative distribution functions (cdfs) of SINR at the BS of picocell $a$ for Scenario 2 in Fig. 4.9. The results show that the combined receiver achieves better SINR compared to the MMSE receiver IA scheme even with correlated antennas. Furthermore, we observe that the combined receiver is less prone to the effects of antenna correlation, as evidenced by the smaller performance degradation in Fig. 4.9.

\subsection{Summary}

Having enough dimensions plays an important role in aligning all interference within a subspace. This is especially very important for the cases where there are multiple users served simultaneously. The multiple users sharing the same radio resources at the same time interfere with each other, degrading the overall system performance. 
In this chapter, we have mainly addressed how to apply IA schemes in the uplink. We have started by discussing the schemes with 2-cell case and then we have shown how to deal with large networks where there are many cells serving many users.

We first started with IA in 2-cell uplink networks. We discussed an IA scheme for such systems, reproduced the results of [100] and [101] and extended these results considering imperfect CSI and limited feedback. We have shown that IEEE 802.16e codebooks could perform better than RVQ codebooks, however imperfect CSI and limited feedback could degrade system performance significantly. Not only did we have discussed the dependency on perfect CSI, we have also looked into the dimensionality challenge. The proposed technique could only accommodate two-cell networks. As the size of the network gets bigger, we need to exploit more dimensions at the transmitter and the receiver. This would result in employing many antennas, however considering the size of the mobile handsets, it is almost impossible to have as many antennas to align all interference at the transmitter side.

In Section 4.1.2, we discussed how to implement IA in an uplink multicell network, where there are more than two cells in the network with many users being served in each cell. We discussed cancelling the interference with existing receiver architectures and addressed the challenges that we encounter with the $\mathrm{ZF}$ and MMSE postcoding vectors. The ZF receiver is required to have enough number of dimensions to cancel the interference, whereas the MMSE receiver needs the knowledge of out-of-cell precoding vectors which is very difficult to obtain in practical systems. Here we categorised the interference as dominant interference and remaining interference and we proposed a novel receiver called the combined receiver which is a combination of the ZF and MMSE postcoders. According to this scheme, the receivers cancel the dominant interference with the ZF part, whereas reduce the remaining interference with MMSE part. We have shown that we can achieve significantly more mean sum rate with our proposed receiver compared to the existing receivers. We also considered antenna correlation here as the combined receiver may require many antennas to be employed at the BS, depending on the number of interferers to be ZF'd. However, as the combined receiver leaves the remaining interference to be handled by the MMSE part, it requires less number of antennas at the receivers compared to the ZF postcoding vectors. 


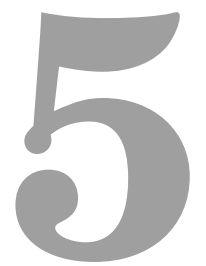

\section{Downlink Interference Alignment and Cancellation in Multicell Networks}

In this chapter, we discuss downlink IAC schemes for multi - user, multi - cellular networks in the downlink. In the first section, we investigate the IAC schemes for the downlink. We discuss IA for downlink 2-cell networks. Following this, we expand the scheme for multicellular networks. Here, we also discuss various alignment schemes such as ZF precoding and SLNR precoding. Furthermore, we study various receivers such as matched filtering (MF), ZF, and the MMSE-like receiver which mimics an MMSE receiver. We then propose a new precoding scheme called the "SLRINR precoding vectors". We also investigate the optimization of a colouring parameter to be introduced later.

Finally in the last section, we discuss cooperation schemes. We propose a partial cooperative interference cancellation scheme based on received powers from adjacent BSs. This enables us to assess the merits of varying mixtures of cooperative and non-cooperative users and the gains while reducing the overhead of CCSI estimation between the interfering BS and the user. We also analytically derive expressions for the additional interference caused by imperfect CCSI and demonstrate its impact on the receiver performance in terms of mean sum rates and the cooperation gains.

We can summarise some of the major contributions of this chapter which are published in [109] and submitted as [110] as follows

- We propose novel precoding vectors which reduce the impact of unknown remaining interference and maximize the SLRINR for each user. Therefore, we refer to these precoders as SLRINR Precoders [110]. 
- We optimize the system colouring parameter in order to reduce the effects of interference and noise [110].

- We propose a partial cooperative IAC scheme based on the received powers from adjacent BSs [109].

- We analyse our system in urban and suburban multicellular environments and demonstrate that the cooperation gains are different for different environments [109].

- We analytically derive expressions for additional interference caused by imperfect CCSI and demonstrate its impact [109].

\subsection{Interference Alignment for 2-Cell Networks in the Downlink}

Here, we consider a simple 2 cell downlink system as the most basic case for IA application in such systems. We can summarise our work in this subsection as follows

- We reproduce and discuss the results of [111].

- We evaluate the simulation results of this system considering imperfect CSI and discuss its impact on the mean sum rates.

We assume 2 users in each cell where the BS is equipped with $N_{t}$ antennas and the receivers have $N_{r}$ antennas. Such system is given in [111], [112]. As seen in Fig. 5.1 the BS $\alpha$ coveys a message $x_{i}$ to each of its own user $i$ through a channel $\mathbf{H}_{\alpha, i} \in \mathcal{C N}(0, \mathbf{I}) \quad\left(N_{r} \times N_{t}\right)$. As there are 2 users in each cell, the BS conveys 2 messages in total. These messages also leak to the user $j$ in cell $\beta$, which is denoted as intercell interference, through a channel $\mathbf{G}_{\beta, j} \in \mathcal{C N}(0, \mathbf{I}) \quad\left(N_{r} \times N_{t}\right)$. The BS $\alpha$ transmits 2 messages through precoding vectors $\mathbf{v}_{\alpha, i}$. The received signal at user $i$ is given as

$$
\mathbf{y}_{i}=\mathbf{H}_{\alpha, i} \sum_{k=1}^{2} \mathbf{v}_{\alpha, k} x_{\alpha, k}+\mathbf{G}_{\alpha, i} \sum_{l=1}^{2} \mathbf{v}_{\beta, l} x_{\beta, l}+\mathbf{n}_{i}
$$

where $\mathbf{n}_{i}$ is the AWGN vector. Then the desired signals are decoded by each user using postcoding vector, $\mathbf{u}_{\alpha, i}$, as follows

$$
\tilde{x}_{\alpha, i}=\mathbf{u}_{\alpha, i}^{*} \mathbf{y}_{\alpha, i}
$$

where the postcoding vector has unit norm, $\left\|\mathbf{u}_{\alpha, i}\right\|=1$. Similar to [100], IA is achieved by the precoding vectors which are carefully generated based on the CSI at the transmitters. 
IAC has been done in 2 steps. The BS first generates the postcoding vectors and then determine the precoding vectors by using the postcoding vectors.

- Step 1: Designing the postcoding vectors The users 1 and 2 in cell $\alpha$ design the postcoding vectors $\mathbf{u}_{\alpha, 1}, \mathbf{u}_{\alpha, 2}$, respectively so that their intercell interference can be aligned.

$$
\operatorname{span}\left(\mathbf{G}_{\alpha, 1}^{*} \mathbf{u}_{\alpha, 1}\right)=\operatorname{span}\left(\mathbf{G}_{\alpha, 2}^{*} \mathbf{u}_{\alpha, 2}\right)
$$

We can find the intersection subspace satisfying the condition (5.3) above by solving the following equation

$$
\left[\begin{array}{ccc}
\mathbf{I}_{N_{r}} & -\mathbf{G}_{\alpha, 1} & \mathbf{0} \\
\mathbf{I}_{N_{r}} & \mathbf{0} & -\mathbf{G}_{\alpha, 2}
\end{array}\right]\left[\begin{array}{c}
\boldsymbol{\eta}_{\alpha}^{I C I} \\
\tilde{\mathbf{u}}_{\alpha, 1} \\
\tilde{\mathbf{u}}_{\alpha, 2}
\end{array}\right]=\mathbf{0} .
$$

Similarly for cell $\beta$

$$
\left[\begin{array}{ccc}
\mathbf{I}_{N_{r}} & -\mathbf{G}_{\beta, 1} & \mathbf{0} \\
\mathbf{I}_{N_{r}} & \mathbf{0} & -\mathbf{G}_{\beta, 2}
\end{array}\right]\left[\begin{array}{c}
\boldsymbol{\eta}_{\beta}^{I C I} \\
\tilde{\mathbf{u}}_{\beta, 1} \\
\tilde{\mathbf{u}}_{\beta, 2}
\end{array}\right]=\mathbf{0}
$$

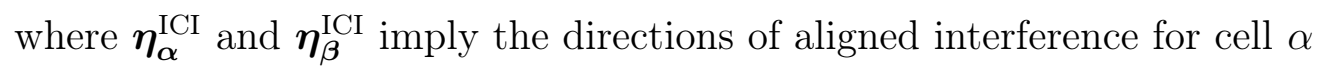
and $\beta$ respectively. Then the postcoding vectors are normalised to have unit norm

$$
\begin{aligned}
& \mathbf{u}_{\alpha, i}=\frac{\tilde{\mathbf{u}}_{\alpha, i}}{\left\|\tilde{\mathbf{u}}_{\alpha, i}\right\|}, \quad i \in\{1,2\} \\
& \mathbf{u}_{\beta, j}=\frac{\tilde{\mathbf{u}}_{\beta, j}}{\left\|\tilde{\mathbf{u}}_{\beta, j}\right\|}, \quad j \in\{1,2\} .
\end{aligned}
$$

- Step 2: Designing the precoding vectors Since the intercell interference vectors are aligned, the BS $\alpha$ can consider two intercell interference vectors as one intercell interference vector which is in the direction of $\boldsymbol{\eta}_{\boldsymbol{\beta}}^{\mathrm{ICI}}$ and spans one dimensional subspace as shown in Fig. 5.1. Therefore, if the precoding vectors $\mathbf{v}_{\alpha, 1}$ and $\mathbf{v}_{\alpha, 2}$ are designed as

$$
\begin{array}{rll}
\tilde{\mathbf{v}}_{\alpha, 1} & \subset \operatorname{null}\left(\left[\left(\mathbf{u}_{\alpha, 2}^{*} \mathbf{H}_{\alpha, 2}\right)^{*}\right.\right. & \left.\left.\boldsymbol{\eta}_{\beta}^{\mathrm{ICI}}\right]\right), \\
\tilde{\mathbf{v}}_{\alpha, 2} & \subset \operatorname{null}\left(\left[\left(\mathbf{u}_{\alpha, 1}^{*} \mathbf{H}_{\alpha, 1}\right)^{*}\right.\right. & \left.\left.\boldsymbol{\eta}_{\beta}^{\mathrm{ICI}}\right]\right) .
\end{array}
$$




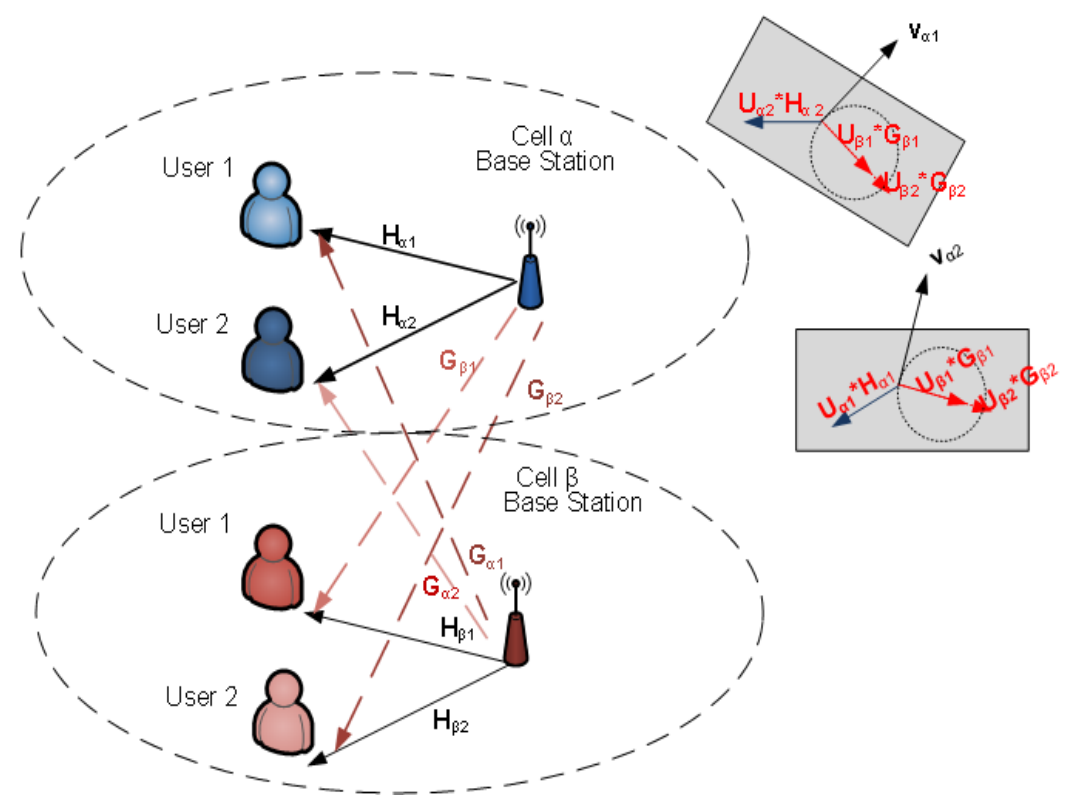

Figure 5.1: System model for 2-cell downlink network of [111]

Similarly, the BS $\beta$ generates the precoding vectors $\mathbf{v}_{\beta, 1}$ and $\mathbf{v}_{\beta, 2}$ as

$$
\begin{aligned}
& \tilde{\mathbf{v}}_{\beta, 1} \subset \operatorname{null}\left(\left[\left(\mathbf{u}_{\beta, 2}^{*} \mathbf{H}_{\beta, 2}\right)^{*} \quad \boldsymbol{\eta}_{\alpha}^{\mathrm{ICI}}\right]\right), \\
& \tilde{\mathbf{v}}_{\beta, 2} \subset \operatorname{null}\left(\left[\left(\mathbf{u}_{\beta, 1}^{*} \mathbf{H}_{\beta, 1}\right)^{*} \quad \boldsymbol{\eta}_{\alpha}^{\mathrm{ICI}}\right]\right) \text {. }
\end{aligned}
$$

Then the precoding vectors are normalised to have unit norms

$$
\begin{aligned}
& \mathbf{v}_{\alpha, i}=\frac{\tilde{\mathbf{v}}_{\alpha, i}}{\left\|\tilde{\mathbf{v}}_{\alpha, i}\right\|}, \quad i \in\{1,2\} \\
& \mathbf{v}_{\beta, j}=\frac{\tilde{\mathbf{v}}_{\beta, j}}{\left\|\tilde{\mathbf{v}}_{\beta, j}\right\|}, \quad j \in\{1,2\} .
\end{aligned}
$$

\section{Imperfect CSI:}

In this case, we assume that the channel matrices used to generate precoding vectors are erroneously estimated. Therefore, considering (2.23), we write the erroneous precoding vectors for cell $\alpha$ and $\beta$ respectively as follows

$$
\begin{aligned}
& \tilde{\hat{\mathbf{v}}}_{\alpha, 1} \subset \operatorname{null}\left(\left[\left(\mathbf{u}_{\alpha, 2}^{*} \hat{\mathbf{H}}_{\alpha, 2}\right)^{*} \quad \boldsymbol{\eta}_{\beta}^{\mathrm{ICI}}\right]\right), \\
& \tilde{\mathbf{v}}_{\alpha, 2} \subset \operatorname{null}\left(\left[\left(\mathbf{u}_{\alpha, 1}^{*} \hat{\mathbf{H}}_{\alpha, 1}\right)^{*} \quad \boldsymbol{\eta}_{\beta}^{\mathrm{ICI}}\right]\right) \text {. } \\
& \tilde{\hat{\mathbf{v}}}_{\beta, 1} \subset \operatorname{null}\left(\left[\left(\mathbf{u}_{\beta, 2}^{*} \hat{\mathbf{H}}_{\beta, 2}\right)^{*} \quad \boldsymbol{\eta}_{\alpha}^{\mathrm{ICI}}\right]\right), \\
& \tilde{\mathbf{v}}_{\beta, 2} \subset \operatorname{null}\left(\left[\left(\mathbf{u}_{\beta, 1}^{*} \hat{\mathbf{H}}_{\beta, 1}\right)^{*} \quad \boldsymbol{\eta}_{\alpha}^{\mathrm{ICI}}\right]\right) .
\end{aligned}
$$


Then the normalised erroneous precoding vectors are given by

$$
\begin{aligned}
\hat{\mathbf{v}}_{\alpha, i}=\frac{\tilde{\hat{\mathbf{v}}}_{\alpha, i}}{\left\|\tilde{\hat{\mathbf{v}}}_{\alpha, i}\right\|}, \quad i \in\{1,2\} \\
\hat{\mathbf{v}}_{\beta, j}=\frac{\tilde{\hat{\mathbf{v}}}_{\beta, j}}{\left\|\tilde{\hat{\mathbf{v}}}_{\beta, j}\right\|}, \quad j \in\{1,2\} .
\end{aligned}
$$

\subsubsection{Performance Metrics}

For 2-cell downlink systems, we give the instantaneous SINR as

$$
\begin{aligned}
& \operatorname{SINR}_{\alpha, i}= \\
& \qquad \frac{\mathbf{u}_{\alpha, i}^{*} \mathbf{H}_{\alpha, i} \mathbf{v}_{\alpha, i} \mathbf{v}_{\alpha, i}^{*} \mathbf{H}_{\alpha, i}^{*} \mathbf{u}_{\alpha, i}}{\sigma^{2}+\mathbf{u}_{\alpha, i}^{*}\left(\sum_{k=1}^{K} \mathbf{G}_{\alpha, i} \mathbf{v}_{\beta, k} \mathbf{v}_{\beta, k}^{*} \mathbf{G}_{\alpha, i}^{*}\right) \mathbf{u}_{\alpha, i}+\mathbf{u}_{\alpha, i}^{*} \mathbf{H}_{\alpha, i}\left(\sum_{j=1, j \neq i}^{K} \mathbf{v}_{\alpha, j} \mathbf{v}_{\alpha, j}^{*}\right) \mathbf{H}_{\alpha, i}^{*} \mathbf{u}_{\alpha, i}} .
\end{aligned}
$$

Then we find the ergodic rate per unit bandwidth for each user $i$ as

$$
\mathrm{R}_{\alpha, i}=\mathbb{E}\left\{\log _{2}\left(1+\operatorname{SINR}_{\alpha, i}\right)\right\}
$$

Finally, we give the ergodic mean sum rate as

$$
\tilde{\mathrm{R}}_{\alpha}=\sum_{i=1}^{K} \mathrm{R}_{\alpha, i}
$$

\section{Simulation Results}

Here we give simulation results for a 2-cell system with 2 users in each cell. We assume the BSs are equipped with $N_{t}=3$ and the users are equipped with $N_{r}=2$ antennas. We also consider imperfect CSI which is given in (2.23), therefore the precoding vectors are erroneous as given in (5.18). We evaluate the simulations in order to find ergodic mean sum rates (5.22) with respect to varying transmit SNRs. The results in Fig. 5.2 show that the system can achieve very good ergodic mean sum rates with increasing transmit SNR. However, introducing channel imperfections, the mean sum rates drop drastically. For example, when the imperfect CSI parameter $\varsigma=0.99$ and at $30 \mathrm{~dB}$ transmit SNR, the system encounter $36 \%$ decrease in mean sum rates. Poorer estimation leads to a dramatical decrease, such that when $\varsigma=0.90$ which is a poor estimation, the mean sum rates at $30 \mathrm{~dB}$ transmit SNR drops to $11 \mathrm{bits} / \mathrm{sec} / \mathrm{Hz}$ from approximately $36 \mathrm{bits} / \mathrm{sec} / \mathrm{Hz}$. This is almost $70 \%$ loss.

Although, the proposed IA technique of [111] achieves very good mean sum rates, it requires extensive information exchange between adjacent cells which 


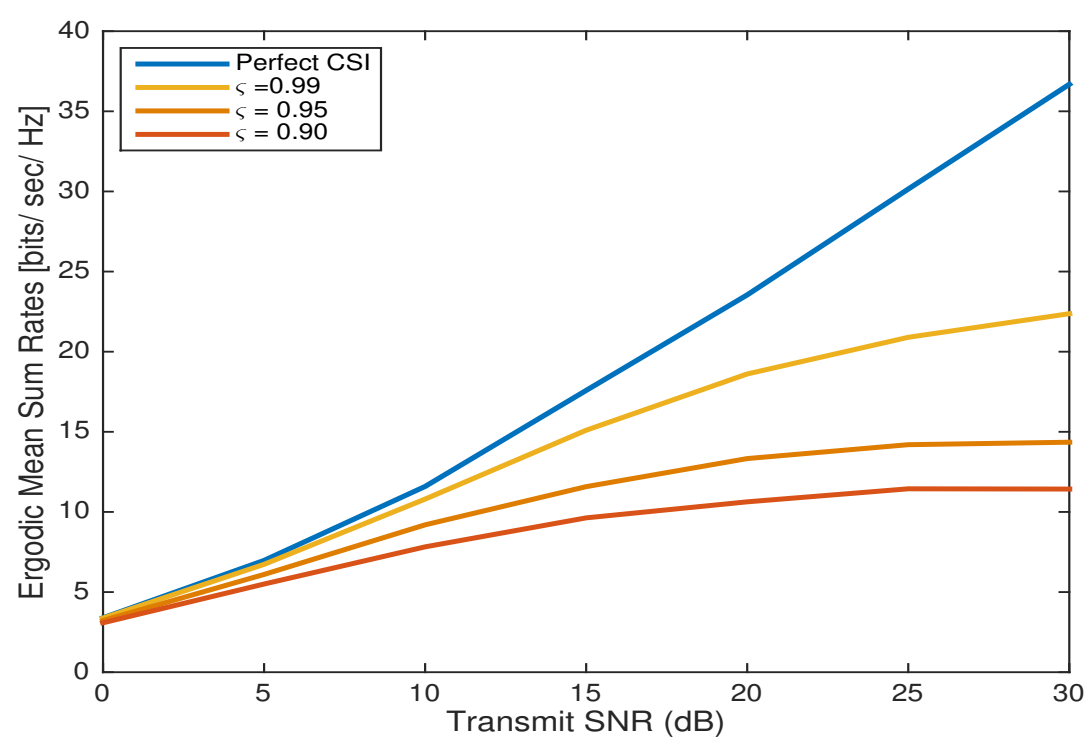

Figure 5.2: Ergodic mean sum rates for 2-cell downlink network with imperfect CSI

is very hard to achieve in practical systems due to the overhead issues. Therefore, the method may not be desirable for larger networks where there are many interfering sources. Furthermore, our results indicate that the proposed technique suffers dramatically from imperfect channel estimation. In the following sections, we investigate methods for larger networks where there are more than 2 cells.

\subsection{Interference Alignment for Multicell Networks in the Downlink}

In this section, we first analyse the IA scheme of [3] which focuses on the implementation of IA for multicell downlink networks. They proposed the implementation of MMSE-like receivers with ZF precoding vectors. In [110], we propose employing precoding vectors based on maximizing the SLNR. We also propose improving these SLNR-based precoding vectors by maximizing the SLRINR. We reduce the effects of interference with an optimised colouring parameter. In addition, we consider imperfect CSI and CCSI. We analyse our system in realistic cellular scenarios given in Chapter 2, considering multiple dominant interferers for randomly located users and also cell-edge users. We demonstrate that our proposed SLRINR precoding vectors outperform the existing precoding vectors. The contributions of this section are as follows

- We propose novel precoding vectors which reduce the impact of unknown remaining interference and also maximise SLRINR for each user. 
- We optimize the system colouring parameter (to be defined later) in order to reduce the effects of interference and noise. We investigate how to improve the sum rates using colouring parameters based on user location within the cell where each user is exposed to different interference levels.

- We also consider the multiple dominant interference case for which we provide simulations using urban macro-cell scenarios using the COST 231 Hata-Urban model and considering random user locations and cell-edge user locations.

- We analyse the impact of imperfect CSI and CCSI and demonstrate that the proposed precoding vectors are more robust to channel estimation errors compared to existing precoding vectors.

We consider a system model similar to downlink IA in [3] as shown in Fig. 5.3 where BS $\alpha$ refers to the desired BS whereas $\beta$ refers to the interfering BS. Each

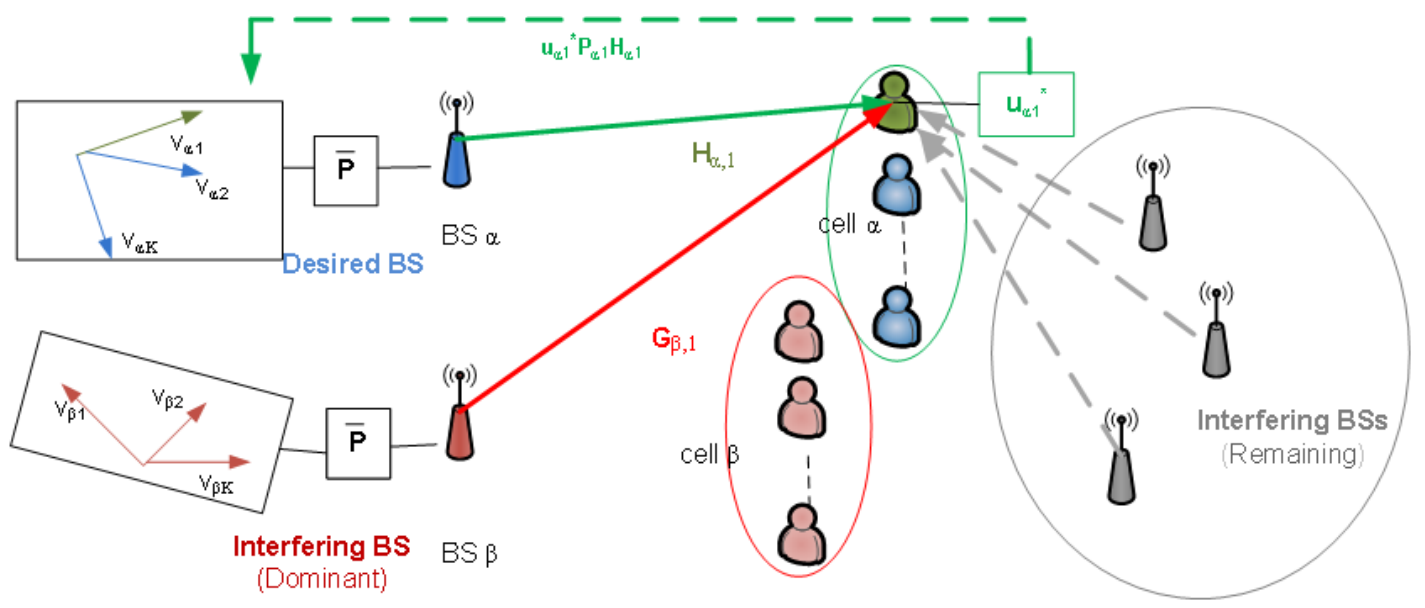

Figure 5.3: System model of downlink IA in multicellular networks of [3]

cell contains $K$ users, each with $N_{r}$ antennas. Each base station (BS) has $N_{t}$ antennas. $S$ is the total number of streams, which is equal to the number of users as it is assumed to be 1 stream per user. The interference can be categorized into two types: intracell interference and intercell interference. Precoding vectors, $\mathbf{v}$, are used to align intracell interference caused by transmissions to other users in the same cell, while postcoding vectors, $\mathbf{u}$, mitigate intercell interference caused by the neighbouring cells. In addition to precoding vectors, an $N_{t}$ by $N_{t}$ secondary precoding matrix $\overline{\mathbf{P}}$ is used at the transmitters to colour the interference space. This fixed precoder matrix is independent of the channel gains.

We note that the system shown in Fig. 5.3 is different from the 2-cell system in Fig. 5.1 because it has multiple interference cells which are considered as remaining interference and dealt with a secodary precoding matrix $\overline{\mathbf{P}}$. However, 
the system of Fig. 5.1 only has 2 cells and do not need a secondary precoding matrix.

Each BS transmits its data using $K$ precoding vectors, for example at BS $\alpha, \mathbf{B}_{\alpha}=\left[\mathbf{v}_{\alpha, 1}, \mathbf{v}_{\alpha, 2}, \ldots, \mathbf{v}_{\alpha, K}\right] \in \mathbb{C}^{N_{t} \times K}$, and the precoding matrix $\overline{\mathbf{P}}$ are used. Using these two precoders, $\overline{\mathbf{P}}$ and $\mathbf{B}_{\alpha}$, BS $\alpha$ sends the complex symbols $\left(x_{\alpha, 1}, x_{\alpha, 2}, \ldots, x_{\alpha, K}\right)$, intended for the users in cell $\alpha$. Furthermore, $\mathbf{H}_{\alpha, k} \in$ $\mathcal{C N}^{N_{r} \times N_{t}}(0, \mathbf{I})$ indicates the direct channel from BS $\alpha$ to user $k$ of cell $\alpha$ and $\mathbf{G}_{\beta, k} \in \mathcal{C N}^{N_{r} \times N_{t}}(0, \mathbf{I})$ denotes the cross channel from BS $\beta$. In a multicell environment, there is more than one interfering cell. Some interfering BSs (intercell interference) are closer to the users in the desired cell and are likely to be dominant compared to the others. The other intercell interferers which have weaker strength are, in aggregate, defined as the remaining interference. In this section, we also apply the approach of [3] to mitigate the remaining interference by treating it as noise. The received signal of user $k$ in cell $\alpha$ is given by

$\mathbf{y}_{\alpha, k}=\left(\sqrt{\frac{\rho_{\alpha, k}}{S}}\right) \mathbf{H}_{\alpha, k} \overline{\mathbf{P}} \sum_{i=1}^{K} \mathbf{v}_{\alpha, i} x_{\alpha, i}+\sum_{\beta}\left(\sqrt{\frac{\rho_{\beta, k}}{S}}\right) \mathbf{G}_{\beta, k} \overline{\mathbf{P}} \sum_{l=1}^{K} \mathbf{v}_{\beta, l} x_{\beta, l}+\mathbf{z}_{\alpha, k}+\mathbf{n}_{\alpha, k}$

where the noise is assumed to be $\mathbf{n}_{\alpha, k} \sim \mathcal{C N}(0, \mathbf{I})$. The dominant interference forms the second term in (5.23) which involves the summation over all dominant BSs indexed by $\beta$. We define $\rho_{\alpha, k}=v \mu_{\alpha, k}$ where $v$ is the total transmit power of the BS and $\mu_{\alpha, k}$ is the attenuation due to path-loss and shadowing. Using (2.2) in Chapter 2, one could write $\mu_{\alpha, k}=10^{\frac{\mathrm{PL}_{[d B]}}{10}} \theta \psi$, where $\theta$ is 3-sector antenna pattern given in $[16], \psi=10^{\left(\tilde{\eta} \sigma_{S F} / 10\right)}$, and $\sigma_{S F}$ is the shadowing standard deviation in dB. $\tilde{\eta}$ is a zero mean Gaussian random variable with unit variance. Because the noise has unit variance, $v$ can also be considered as the transmit $\mathrm{SNR}^{1}$ as in [3]. Note that, we assume a single dominant interferer as in [3], then we extend our work to multiple dominant interferers. The variable $\mathbf{z}_{\alpha, k}$ denotes the remaining interference from all other, non-dominant cells. We parametrize the system with $\gamma=\frac{\mathrm{INR}_{\text {rem }}}{\mathrm{INR}_{\mathrm{dom}}}$, where $\mathrm{INR}_{\mathrm{dom}}$ and $\mathrm{INR}_{\text {rem }}$ are the long term interference to noise ratios of the total dominant and remaining interference respectively.

\footnotetext{
${ }^{1}$ Note that SNR is a value that should be derived at the receiver. However, in this case $v$ can be considered as logically equivalent to an SNR as the noise variance is unity. Therefore we refer to it as transmit SNR. This concept is also adopted in [3] and in a number of other papers.
} 


\subsubsection{Design of the Receivers}

Receiver design is vital for mitigating intercell interference. The strength of the remaining interference plays a key role in designing the receiver. If the remaining interference is small, or negligible compared to the dominant interference, ZF receivers provide significant gains over MF receivers [3]. The user $k$ in cell $\alpha$ estimates the interference channel of the neighbouring cell, $\mathbf{G}_{\beta, k} \overline{\mathbf{P}}$ using pilots or a preamble and then it generates a postcoding vector, $\mathbf{u}_{\alpha, k}$, with which the received vector is post-processed as $\mathbf{u}_{\alpha, k}^{*} \mathbf{y}_{\alpha, k}$. In order to cancel intercell interference, the ZF postcoding vector can be generated such that $\mathbf{u}_{\alpha, k}^{(Z F) *} \mathbf{G}_{\beta, k} \overline{\mathbf{P}}=0$. However, if some interferers in the network are of similar power levels or the total remaining interference is stronger than the dominant interference, the MF receiver outperforms the $\mathrm{ZF}$ receiver [3]. The MF receiver

uses the dominant left singular vector of the direct channel so that $\mathbf{u}_{\alpha, k}^{(M F)}$ is given by the maximum left singular vector of $\mathbf{H}_{\alpha, k}$.

For a multicell multi-user network where there are interferers coming from more than one cell, assuming the presence of a dominant interferer, an MMSE-like receiver is proposed in [3]. The concept of MMSE-like IA consists of three parts: (a) colouring an interference space, (b) designing a colouring parameter, $\kappa$, to colour the interference space and (c) designing an MMSE-like receiver based on this colouring parameter. The term colouring in this section is similar to the idea of colouring the white noise (i.e. White noise has same power over all frequency bands, while coloured noise has different power over different frequency bands). Similarly, the colouring interference signals in [3] is based on weighting each stream considering remaining and dominant interference. We control the colouredness of interference signals by using a secondary precoding matrix $\overline{\mathbf{P}}$, differently weighting the last $\left(N_{t}-S\right)$ columns of an arbitrary unitary matrix with a colouring parameter $\kappa . \overline{\mathbf{P}}$ is given in [3]

$$
\overline{\mathbf{P}}=\tilde{\boldsymbol{\Psi}}\left[\begin{array}{cc}
\mathbf{I}_{S} & 0 \\
0 & \kappa \mathbf{I}_{N_{t}-S}
\end{array}\right]
$$

where $\tilde{\boldsymbol{\Psi}}$ is an arbitrary $N_{t} \times N_{t}$ unitary matrix.

In [3], $\kappa$ is found heuristically based on simulation results where it was found that a suitable $\kappa$ given by

$$
\kappa=\min \left(\gamma^{1 / 4}, 1\right)
$$

The authors of [3] suggested to use different colouring parameter, $\kappa$ for different scenarios, however they did not provide any solution regarding to this. In the following section, we will show that the $\kappa$, can be optimised to achieve a better performance than that given by (5.25). For the MMSE-like receiver, the 
covariance matrix of interference plus noise at user $k$ in cell $\alpha$ is given by [3]

$$
\boldsymbol{\Phi}_{\alpha, k}=\left(1+\mathrm{INR}_{\mathrm{rem}}\right) \mathbf{I}+\frac{\rho_{\beta, k}}{S}\left(\mathbf{G}_{\beta, k} \overline{\mathbf{P}} \mathbf{B}_{\beta} \mathbf{B}_{\beta}^{*} \overline{\mathbf{P}}^{*} \mathbf{G}_{\beta, k}^{*}\right)
$$

where $\mathbf{B}_{\beta}=\left[\mathbf{v}_{\beta, 1}, \ldots, \mathbf{v}_{\beta, K}\right]$ indicates the $\mathrm{ZF}$ precoding vector of a dominant interferer $(\mathrm{BS} \beta)$. Note that (5.26) is for the case where only one of the neighbouring cells causes dominant interference. It can be expanded for the cases where there are multiple dominant interfering cells as discussed earlier. Since the system is uncoordinated, $\mathbf{B}_{\beta}$ is unknown to each user in cell $\alpha$ and therefore $\boldsymbol{\Phi}_{\alpha, k}$ cannot be computed. Consequently, the expected value of the covariance matrix is considered

$\overline{\mathbf{\Phi}}_{\alpha, k}:=\mathbb{E}\left[\boldsymbol{\Phi}_{k}\right]=\left(1+\mathrm{INR}_{\mathrm{rem}}\right) \mathbf{I}+\frac{\rho_{\beta, k}}{S+\left(N_{t}-S\right) \kappa^{2}}\left(\mathbf{G}_{\beta, k} \overline{\mathbf{P}}\left[\begin{array}{cc}\mathbf{I}_{S} & \mathbf{0} \\ \mathbf{0} & \kappa^{2} \mathbf{I}_{N_{t}-S}\end{array}\right] \overline{\mathbf{P}}^{*} \mathbf{G}_{\beta, k}^{*}\right)$,

where the expectation is performed over $\mathbf{B}_{\beta} . \mathbb{E}\left[\mathbf{B}_{\beta} \mathbf{B}_{\beta}^{*}\right]=\frac{S}{S+\left(N_{t}-S\right) \kappa^{2}}\left[\begin{array}{cc}\mathbf{I}_{S} & \mathbf{0} \\ \mathbf{0} & \kappa^{2} \mathbf{I}_{N_{t}-S}\end{array}\right]$ is given in [3] for ZF precoding vectors. Because the value of $\mathbb{E}\left[\mathbf{B}_{\beta} \mathbf{B}_{\beta}^{*}\right]$ with SLNR precoding vectors involves the maximum eigenvector of a matrix, it is very difficult to derive a close-form formula for the expectation of the SLNRbased precoding vectors. Via simulations, we find that the expectation of the SLNR-based precoders approximates that of ZF precoders where the transmit SNR is high (interference limited region). The findings in references [113] and [114] also support our simulation results. [113] indicates that regularized ZF (RZF) is same as ZF precoders at high transmit SNR. Also [114] proves that the SLNR precoder is equivalent to the RZF. Therefore, considering our simulation results and the references [113] and [114], we use the expectation given in [3] for our SLNR-based precoding vectors. Then the MMSE-like postcoding vectors are found as follows

$$
\mathbf{u}_{\alpha, i}=\frac{\left(\overline{\mathbf{\Phi}}_{\alpha, k}\right)^{-1} \mathbf{H}_{\alpha, k} \overline{\mathbf{P}}_{\mathbf{v}_{\alpha, k}}}{\|\left(\overline{\boldsymbol{\Phi}}_{\alpha, k}\right)^{-1} \mathbf{H}_{\alpha, k} \overline{\mathbf{P}}_{\mathbf{v}_{\alpha, k} \|}}
$$

The authors of [3] proposed an iterative algorithm to design an MMSE-like receiver, incorporating an opportunistic scheduler. According to this algorithm, each user first initializes its postcoding vector, $\mathbf{u}_{\alpha, k}$. Then, precoding vectors, $\mathbf{v}_{\alpha, k}$, are found and postcoding vectors are updated. Note that (5.27) does not change with the iteration as it is an expectation. However, generating 
postcoding and precoding vectors requires feedback between the transmitter and receiver, therefore iteration would extensively increase the overhead. Therefore, we do not consider any iteration.

\subsubsection{Selecting The Colouring Parameter $\kappa$}

In [3], it is assumed that all users are located in the same region within the cell, so that they experience the same amount of interference from the other cells. Therefore, the same $\kappa$ is used for all users.

However, users have variable locations and therefore their interference ratio, $\gamma$, and hence $\kappa$ should also change. The MMSE-like receiver mimics ZF when the remaining interference is negligible, $\gamma \ll 1$, and adjusts itself to mimic MF when there are no dominant interferers, $\gamma \gg 1$. Here, we provide information how $\kappa$ impacts the system for the extreme cases of the $\gamma$ ratios.

- $\gamma \gg 1$ : In this case, $\kappa=1$, so remaining interference is equal to dominant interference. In other words, there is no dominant interference. Therefore MMSE-like receiver of which weight vector given by $\frac{\overline{\mathbf{\Phi}}_{\alpha, k}^{-1} \mathbf{H}_{\alpha, k} \overline{\mathbf{P}} \mathbf{v}_{\alpha, k}}{\left\|\overline{\mathbf{\Phi}}_{\alpha, k}^{-1} \mathbf{H}_{\alpha, k} \overline{\mathbf{P}}_{\alpha, k}\right\|}$, should mimic the MF where $\overline{\mathbf{\Phi}}_{\alpha, k} \approx \mathbf{I}$.

Substituting $\kappa=1$ in (5.27), we get

$$
\overline{\mathbf{\Phi}}_{k}=\left(1+\mathrm{INR}_{\mathrm{rem}}\right) \mathbf{I}+\frac{\rho_{\beta, k}}{N_{t}} \tilde{\mathbf{G}}_{\beta, k} \tilde{\mathbf{G}}_{\beta, k}^{*}
$$

where $\tilde{\mathbf{G}}_{\beta, k}$ is an equivalent channel $\left(\mathbf{G}_{\beta, k} \tilde{\boldsymbol{\Psi}}\right), \tilde{\boldsymbol{\Psi}}$ is a unitary matrix defined in $(5.24)$. Note that $\mathbb{E}\left(\frac{\tilde{\mathbf{G}} \tilde{\mathbf{G}}^{*}}{N_{t}}\right)=\mathbf{I}$ so on average $\overline{\boldsymbol{\Phi}}_{k} \approx \mathbf{I}$.

- $\gamma \ll 1$ In this case $\kappa=0$, this means dominant interference is so strong and the remaining interference is negligible. So the covariance matrix of interference and noise can be written as

$$
\overline{\mathbf{\Phi}}=\left(1+\mathrm{INR}_{\mathrm{rem}}\right) \mathbf{I}+\frac{\rho_{\beta, k}}{S} \mathbf{G}_{\beta, k}\left[\tilde{\boldsymbol{\Psi}}_{S} 0\right]\left[\begin{array}{cc}
\mathbf{I}_{S} & 0 \\
0 & 0
\end{array}\right]\left[\begin{array}{c}
\tilde{\boldsymbol{\Psi}}_{S} \\
0
\end{array}\right] \mathbf{G}_{\beta, k}^{*} .
$$

In this case, IA with $\mathrm{ZF}$ receivers outperform the other techniques because the dominant interferers can be nulled properly at receivers and the remaining interferers are negligible. In this case the other receivers can not provide better performance than $\mathrm{ZF}$ receivers because of the following reasons

- ZF receivers do not need information of the out-of-cell precoders to null intercell interference, but MMSE receivers do need them for perfect cancellation. However, it is very difficult to get this information from all interfering users, that is why we assume that the information 
of the out-of-cell precoders are not available. Therefore ZF receivers perform better than MMSE receivers under this assumption.

- MF receivers do not cancel intercell interference directly.

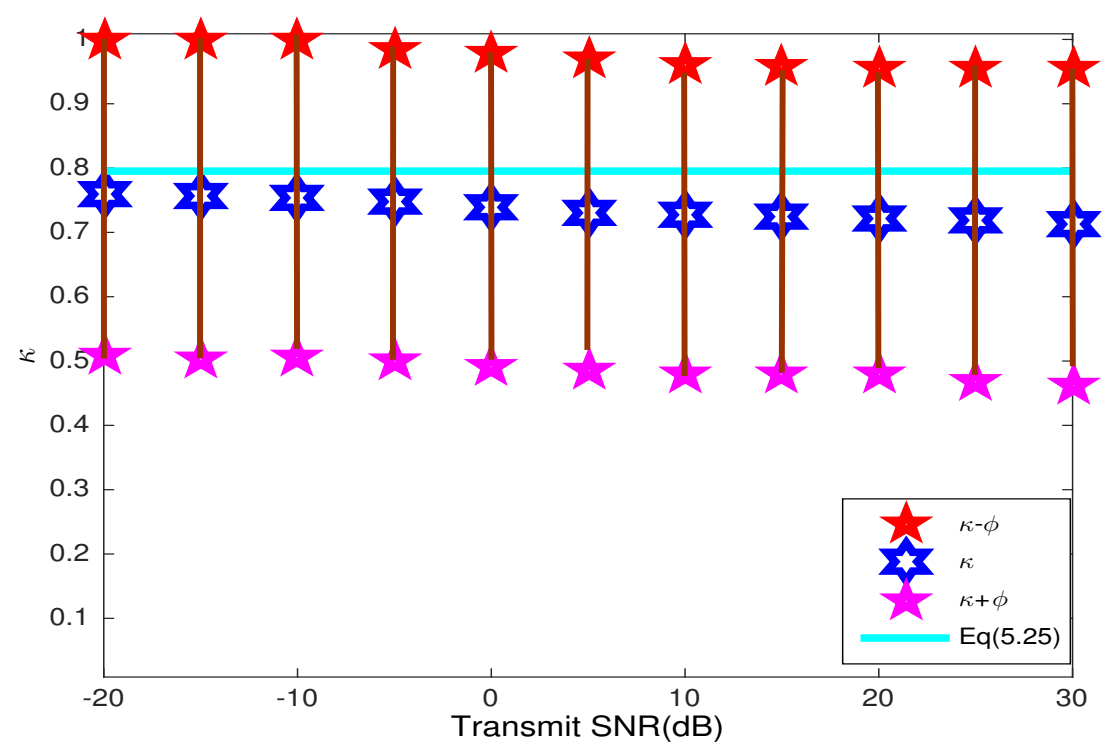

Figure 5.4: Simulations for $\gamma=0.4$ showing the variation in optimal $\kappa$ values. $\kappa$ computed from (5.25) is also shown

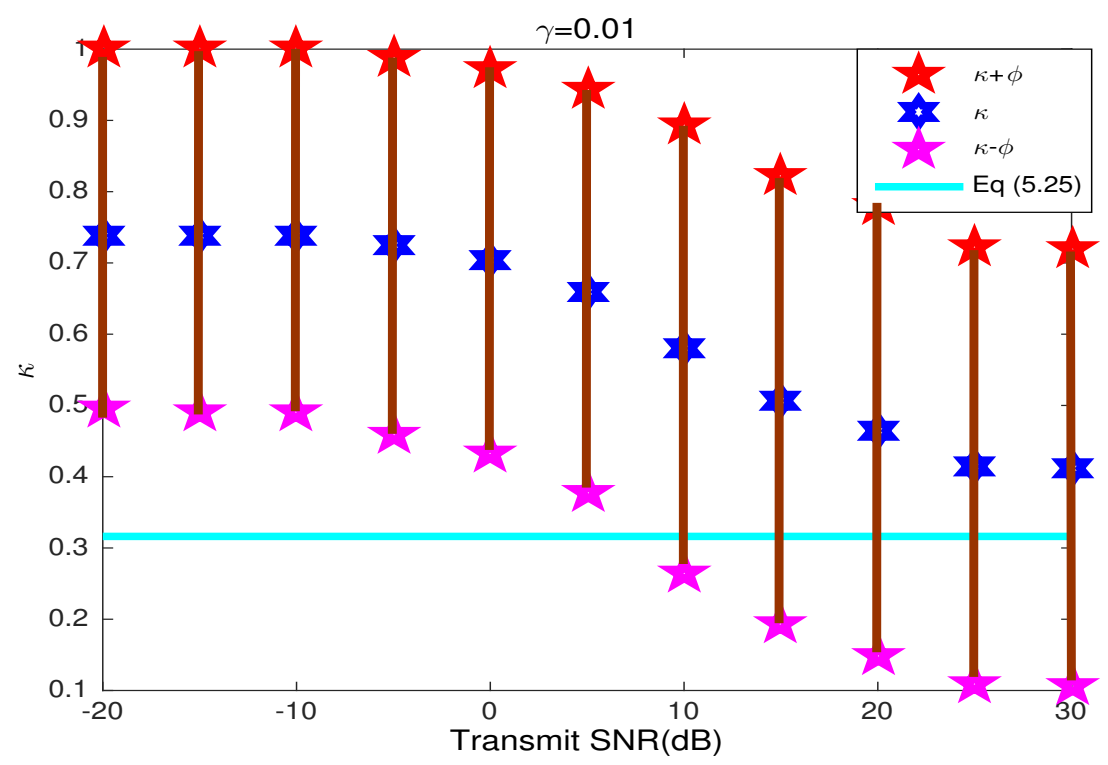

Figure 5.5: Simulations for $\gamma=0.01$ showing the variation in optimal $\kappa$ values. $\kappa$ computed from (5.25) is also shown

Since $\kappa$ impacts the SINR, each user's capacity can be increased by selecting $\kappa$ depending on the location. As a result, the way that each receiver handles 
the interference should change according to the user's location. Note that $\kappa$ found from (5.25) gives approximate results to maximise the mean sum rate but not the exact maximum as seen in Fig. 5.4 and Fig. 5.5. These results show a gap between the mean sum capacity for a fixed $\kappa$ derived heuristically from (5.25) and a $\kappa$ derived via simulations that maximises the sum rate. We have evaluated the simulations for two cases in which remaining interference is significantly strong $(\gamma=0.4)$ and significantly weak $(\gamma=0.01)$. The simulations shown are for $N_{t}=N_{r}=4$ and $S=3$.

In Fig. 5.4 and Fig. 5.5, the mean $\kappa$ value which maximised the sum rates is given. We also give the values at one standard deviation $(\phi)$ higher and lower to show the range of $\kappa$. As seen in Fig. 5.5, there is a gap between the $\kappa$ that maximises the sum rate and the $\kappa$ that is found in Eq. 5.25. This gap is more prominent for lower $\gamma$ ratios, however it is very close to actual values for higher $\gamma$ ratios.

We propose to improve these results by finding $\kappa$ values that reduce the interference for each user at each instantaneous channel. This improves the received SINR and hence the sum rate. Maximizing the sum rate itself is a very complex problem because a closed-form expression for sum rate cannot be written due to the fact that maximum eigenvectors are used in the precoding vectors. Therefore, our approach to selecting $\kappa$ is based on reducing the power of interference and noise which is the trace of (5.27).

The power of interference and noise is

$$
\begin{aligned}
\Xi & =\operatorname{tr}\left(\overline{\mathbf{\Phi}}_{\alpha, k}\right) \\
& =\operatorname{tr}\left(\left(1+\mathrm{INR}_{\mathrm{rem}}\right) \mathbf{I}+\frac{\rho_{\beta, k}}{S+\left(N_{t}-S\right) \kappa^{2}}\left(\mathbf{G}_{\beta, k} \overline{\mathbf{P}}\left[\begin{array}{cc}
\mathbf{I}_{S} & 0 \\
0 & \kappa^{2} \mathbf{I}_{N_{t}-S}
\end{array}\right] \overline{\mathbf{P}}^{*} \mathbf{G}_{\beta, \mathbf{k}}^{*}\right)\right)
\end{aligned}
$$

Then we rewrite $\Xi$ as

$$
\Xi=\left(1+\mathrm{INR}_{\mathrm{rem}}\right) N_{t}+\frac{\rho_{\beta, k}}{S+\left(N_{t}-S\right) \kappa^{2}}\left(A+B \kappa^{4}\right),
$$

where

$$
\begin{aligned}
& A=\left\|\widetilde{\mathbf{G}}_{\beta, k}\right\|_{F}^{2} \\
& B=\left\|\widehat{\mathbf{G}}_{\beta, k}\right\|_{F}^{2},
\end{aligned}
$$

where $\widetilde{\mathbf{G}}_{\beta, k}$ contains the first $S$ columns of $\mathbf{G}_{\beta, k} \boldsymbol{\Psi}$ and $\widehat{\mathbf{G}}_{\beta, k}$ contains the last $N_{t}-S$ columns of $\mathbf{G}_{\beta, k} \boldsymbol{\Psi}$. \|\|$_{F}$ denotes the Frobenius norm. $\boldsymbol{\Psi}$ is any unitary matrix used to generate $\overline{\mathbf{P}}$. Now we can minimise $\Xi$ with respect to $\kappa$ by setting 
the first derivative of $\Xi$ to zero giving

$$
\kappa=\sqrt{\frac{-B S+\sqrt{B^{2} S^{2}+B A\left(N_{t}-S\right)^{2}}}{B\left(N_{t}-S\right)}} .
$$

Hence, the proposed colouring parameter is $\tilde{\kappa}$ where $\tilde{\kappa}=\min (\kappa, 1)$. Note that $\kappa$ found heuristically using (5.25) is only dependent on the $\gamma$ ratio and is fixed for all channel drops. However, the optimised $\tilde{\kappa}$ is also dependent on the cross channel information, and needs to be updated for each channel drop. This would add to the complexity of the system.

\subsubsection{Generating Precoding Vectors}

In this section, we study SLNR precoding vectors due to their improved performance relative to the ZF [92]. The study in [92] considered a single cell and we extend this to a multicell network. We also propose improving the SLNR-based precoders by considering the remaining interference and maximizing SLRINR for each user. We denote these precoders as SLRINR precoding vectors.

- ZF-Based Precoding Vectors ZF precoders are computed by [3]

$$
\left[\begin{array}{llll}
\mathbf{v}_{\alpha, 1} & \mathbf{v}_{\alpha, 2} & \ldots & \mathbf{v}_{\alpha, K}
\end{array}\right]=\mathbf{H}^{*}\left(\mathbf{H H}^{*}\right)^{-1},
$$

where $\mathbf{H}=\left[\mathbf{u}_{\alpha, 1}^{*} \mathbf{H}_{\alpha, 1} \overline{\mathbf{P}} ; \ldots ; \mathbf{u}_{\alpha, K}^{*} \mathbf{H}_{\alpha, K} \overline{\mathbf{P}}\right]$. The precoders are then normalised, such that $\left\|\mathbf{v}_{\alpha, i}\right\|=1$.

- SLNR-Based Precoders In [92], the use of transmit precoding vectors based on the concept of signal leakage was proposed. We now consider employing such precoders in the system of [3]. To analyse the SLNR based precoders, we first write the postcoded received signal at user $k$ of cell $\alpha$ as

$$
\begin{aligned}
& \tilde{\mathbf{x}}_{\alpha, k}=\underbrace{\sqrt{\frac{\rho_{\alpha, k}}{S}} \mathbf{u}_{\alpha, k}^{*} \mathbf{H}_{\alpha, k} \overline{\mathbf{P}} \mathbf{v}_{\alpha, k} x_{\alpha, k}}_{\text {Desired Signal }} \\
& +\sum_{j=1, j \neq k}^{K} \underbrace{\sqrt{\frac{\rho_{\alpha, k}}{S}} \mathbf{u}_{\alpha, k}^{*} \mathbf{H}_{\alpha, k} \overline{\mathbf{P}} \mathbf{v}_{\alpha, j} x_{\alpha, j}}_{\text {Intracell Interference }} \\
& +\underbrace{\sqrt{\frac{\rho_{\beta, k}}{S}} \mathbf{u}_{\alpha, k}^{*} \mathbf{G}_{\beta, k} \overline{\mathbf{P}} \sum_{i=1}^{K} \mathbf{v}_{\beta, i} x_{\beta, i}}_{\text {Intercell Interference }}+\mathbf{u}_{\alpha, k}^{*} \mathbf{z}_{\alpha, k}+\mathbf{u}_{\alpha, k}^{*} \mathbf{n}_{\alpha, k} .
\end{aligned}
$$

SLNR-based precoders aim to maximise signal power over leaked intracell interference and noise. The power of interference that is "leaking" from 
the desired user $(k)$ to user $(j)$ is given by $\left|\sqrt{\frac{\rho_{\alpha, j}}{S}} \mathbf{u}_{\alpha, j}^{*} \mathbf{H}_{\alpha, j} \overline{\mathbf{P}} \mathbf{v}_{\alpha, k}\right|^{2}$. Thus the SLNR parameter for user $k$ is given by

$$
\operatorname{SLNR}_{\alpha, k}=\frac{\left|\sqrt{\frac{\rho_{\alpha, k}}{S}} \mathbf{u}_{\alpha, k}^{*} \mathbf{H}_{\alpha, k} \overline{\mathbf{P}} \mathbf{v}_{\alpha, k}\right|^{2}}{1+\sum_{j=1, j \neq k}^{K}\left|\sqrt{\frac{\rho_{\alpha, j}}{S}} \mathbf{u}_{\alpha, j}^{*} \mathbf{H}_{\alpha, j} \overline{\mathbf{P}} \mathbf{v}_{\alpha, k}\right|^{2}}
$$

We select precoding vectors $\mathbf{v}_{\alpha, k}, k=\{1,2,3, \ldots, K\}$, such that (5.37) is maximised over $\mathbf{v}_{\alpha, k}$, subject to $\left\|\mathbf{v}_{\alpha, k}\right\|=1$. In doing so, (5.37) can be rewritten as

$$
\operatorname{SLNR}_{\alpha, k}=\frac{\left(\frac{\rho_{\alpha, k}}{S}\right) \mathbf{v}_{\alpha, k}^{*} \overline{\mathbf{P}}^{*} \mathbf{H}_{\alpha, k}^{*} \mathbf{u}_{\alpha, k} \mathbf{u}_{\alpha, k}^{*} \mathbf{H}_{\alpha, k} \overline{\mathbf{P}} \mathbf{v}_{\alpha, k}}{1+\mathbf{v}_{\alpha, k}^{*} \overline{\mathbf{P}}^{*} \tilde{\mathbf{H}}_{\alpha, k}^{*} \tilde{\mathbf{H}}_{\alpha, k} \overline{\mathbf{P}}_{\mathbf{v}_{\alpha, k}}},
$$

where

$$
\begin{gathered}
\tilde{\mathbf{H}}_{\alpha, k}=\left[\mathbf{F}_{\alpha, 1}^{T} \mathbf{F}_{\alpha, 2}^{T} \ldots \mathbf{F}_{\alpha, k-1}^{T} \mathbf{F}_{\alpha, k+1}^{T} \ldots \mathbf{F}_{\alpha, K}^{T}\right]^{T} \\
\text { and } \mathbf{F}_{\alpha, i}=\sqrt{\frac{\rho_{\alpha, i}}{S}} \mathbf{u}_{\alpha, i}^{*} \mathbf{H}_{\alpha, i} .
\end{gathered}
$$

$\tilde{\mathbf{H}}_{\alpha, k}$ is an extended equivalent channel which excludes only $\mathbf{u}_{\alpha, k}^{*} \mathbf{H}_{\alpha, k}$. It was shown in [115] that the vector, $\mathbf{v}_{\alpha, k}$, which maximises the SLNR is the leading eigenvector of $\left(\left(\mathbf{I}+\overline{\tilde{\mathbf{H}}}_{\alpha, k}^{*} \overline{\tilde{\mathbf{H}}}_{\alpha, k}\right)^{-1} \overline{\mathbf{H}}_{\alpha, k}^{*} \overline{\mathbf{H}}_{\alpha, k}\right)$, where

$$
\begin{aligned}
\overline{\tilde{\mathbf{H}}}_{\alpha, k} & =\tilde{\mathbf{H}}_{\alpha, k} \overline{\mathbf{P}} \\
\overline{\mathbf{H}}_{\alpha, k} & =\sqrt{\frac{\rho_{\alpha, k}}{S}} \mathbf{u}_{\alpha, k}^{*} \mathbf{H}_{\alpha, k} \overline{\mathbf{P}} .
\end{aligned}
$$

\section{- SLRINR-Based Precoders}

As the transmit SNR increases, the system becomes interference limited. In particular, the remaining interference is the main limitation as the receiver cannot handle it very efficiently. In [3], it is assumed that the power of the remaining interference is constant for each transmit SNR, independent of the dominant interfering channel, and known at the transmitters. Hence in [3], user selection is proposed using estimated rates and considering the remaining interference powers. Using remaining interference knowledge at the transmitters, we propose new precoders based on 
maximizing the SLRINR which can be defined as

$$
\operatorname{SLRINR}_{\alpha, k}=\frac{\left|\sqrt{\frac{\rho_{\alpha, k}}{S}} \mathbf{u}_{\alpha, k}^{*} \mathbf{H}_{\alpha, k} \overline{\mathbf{P}} \mathbf{v}_{\alpha, k}\right|^{2}}{\left(1+\mathrm{INR}_{\mathrm{rem}}\right)+\sum_{j=1, j \neq k}^{K} \mid \sqrt{\frac{\rho_{\alpha, j}}{S}} \mathbf{u}_{\alpha, j}^{*} \mathbf{H}_{\alpha, j} \overline{\mathbf{P}}_{\left.\mathbf{v}_{\alpha, k}\right|^{2}}}
$$

We select precoding vectors $\mathbf{v}_{\alpha, k}, k=\{1,2,3, \ldots, K\}$, such that (5.42) is maximised over $\mathbf{v}_{\alpha, k}$, subject to $\left\|\mathbf{v}_{\alpha, k}\right\|=1$.

Following the previous section, the vector, $\mathbf{v}_{\alpha, k}$, which maximises the SLRINR can be found as the leading eigenvector of

$$
\left(\left(\left(1+\mathrm{INR}_{\mathrm{rem}}\right) \mathbf{I}+\overline{\tilde{\mathbf{H}}}_{\alpha, k}^{*} \overline{\tilde{\mathbf{H}}}_{\alpha, k}\right)^{-1} \overline{\mathbf{H}}_{\alpha, k}^{*} \overline{\mathbf{H}}_{\alpha, k}\right) .
$$

\subsubsection{Considering Multiple Dominant Interferers}

Dominant interference can be due to a single interfering cell or multiple interfering cells. Hence, we extend [3] to multiple dominant interferers, where (5.26) can be written as

$$
\boldsymbol{\Phi}_{\alpha, k}=\mathbb{E}\left[\left(1+\mathrm{INR}_{\mathrm{rem}}\right) \mathbf{I}+\sum_{\beta}\left(\frac{\rho_{\beta, k}}{S} \mathbf{G}_{\beta, k} \overline{\mathbf{P}} \mathbf{B}_{\beta} \mathbf{B}_{\beta}^{*} \overline{\mathbf{P}}^{*} \mathbf{G}_{\beta, k}^{*}\right)\right] .
$$

In (5.43), $\mathbf{G}_{\beta, k}$ denotes cross-channels from all dominant interfering BSs which are indexed by $\beta$.

\section{The Algorithm}

We now describe the algorithm for the proposed IAC scheme.

- Generating postcoding vectors MMSE postcoding vectors need precoders as given in (5.28). All precoders, ZF, SLNR and SLRINR-based precoding vectors also need postcoding vectors (See Eqs. (5.35), (5.37) and (5.42)). Therefore, the users first initialise their postcoding vectors as

$$
\mathbf{u}_{\alpha, i}^{(0)}=\left(\overline{\mathbf{\Phi}}_{\alpha, i}\right)^{-1} \mathbf{H}_{\alpha, i} \overline{\mathbf{P}}_{\mathbf{v}_{\alpha, i}^{(0)}}
$$

where we set $\mathbf{v}_{\alpha, i}^{(0)}$ as the maximum eigenvector of $\overline{\mathbf{P}}^{*} \mathbf{H}_{\alpha, i}^{*} \overline{\boldsymbol{\Phi}}_{\alpha, i}^{-1} \mathbf{H}_{\alpha, i} \overline{\mathbf{P}}$. The initial postcoding vectors are also normalised to have unit norm, $\left\|\mathbf{u}_{\alpha, i}^{(0)}\right\|=$ 1.

- Generating Precoding Vectors Depending on the precoding type, the $\mathrm{BS}$ computes the precoding vectors. 
- ZF Precoders

$$
\left[\begin{array}{llll}
\mathbf{v}_{\alpha, 1}^{(1)} & \mathbf{v}_{\alpha, 2}^{(1)} & \ldots & \mathbf{v}_{\alpha, k}^{(1)}
\end{array}\right]=\mathbf{H}^{(\mathbf{0})^{*}}\left(\mathbf{H}^{(\mathbf{0})} \mathbf{H}^{(\mathbf{0})^{*}}\right)^{-1}
$$

where $\mathbf{H}^{(0)}=\left[\mathbf{u}_{\alpha, 1}^{(0) *} \mathbf{H}_{\alpha, 1} \overline{\mathbf{P}} ; \ldots ; \mathbf{u}_{\alpha, K}^{(0) *} \mathbf{H}_{\alpha, K} \overline{\mathbf{P}}\right]$.

- SLNR Precoders $\mathbf{v}_{\alpha, i}^{(1)}$, is the leading eigenvector of

$$
\begin{aligned}
& \left(\mathbf{I}+\tilde{\mathbf{H}}_{\alpha, i}^{(0) *} \tilde{\mathbf{H}}_{\alpha, i}^{(0)}\right)^{-1}\left(\frac{\rho_{\alpha, i}}{S}\right) \overline{\mathbf{P}}^{*} \mathbf{H}_{\alpha, i}^{*} \mathbf{u}_{\alpha, i}^{(0)} \mathbf{u}_{\alpha, i}^{(0) *} \mathbf{H}_{\alpha, i} \overline{\mathbf{P}} \text {, where } \\
& \tilde{\mathbf{H}}_{\alpha, i}^{(0)}=\left[\begin{array}{llllll}
\mathbf{F}_{\alpha, 1}^{(0)^{T}} \mathbf{F}_{\alpha, 2}^{(0)^{T}} & \ldots & \mathbf{F}_{\alpha, i-1}^{(0)^{T}} \mathbf{F}_{\alpha, i+1}^{(0)^{T}} & \ldots & \mathbf{F}_{\alpha, K}^{(0)^{T}}
\end{array}\right]^{T} \\
& \text { and } \mathbf{F}_{\alpha, i}^{(0)}=\sqrt{\frac{\rho_{\alpha, i}}{S}} \mathbf{u}_{\alpha, i}^{(0)^{*}} \mathbf{H}_{\alpha, i} \overline{\mathbf{P}} \text {. }
\end{aligned}
$$

- SLRINR Precoders $\mathbf{v}_{\alpha, i}^{(1)}$, is the leading eigenvector of

$$
\left(\left(1+\operatorname{INR}_{\mathrm{rem}}\right) \mathbf{I}+\tilde{\mathbf{H}}_{\alpha, i}^{(0) *} \tilde{\mathbf{H}}_{\alpha, i}^{(0)}\right)^{-1}\left(\frac{\rho_{\alpha, i}}{S}\right) \overline{\mathbf{P}}^{*} \mathbf{H}_{\alpha, i}^{*} \mathbf{u}_{\alpha, i}^{(0)} \mathbf{u}_{\alpha, i}^{(0) *} \mathbf{H}_{\alpha, i} \overline{\mathbf{P}}
$$

subject to $\left\|\mathbf{v}_{\alpha, k}^{(1)}\right\|=1$. Then the BS use the precoders generated. In [3], iteration between the BS and the users was applied to update the postcoders and precoders. However, we don't use iteration in this work as it would increase the overhead dramatically.

\subsubsection{Performance Metrics}

SINR for user $k$ in cell $\alpha$ is defined as

$$
\operatorname{SINR}_{\alpha, k}=\frac{\left(\frac{\rho_{\alpha, k}}{S}\right) \mathbf{u}_{\alpha, k}^{*} \mathbf{H}_{\alpha, k} \overline{\mathbf{P}} \mathbf{v}_{\alpha, k} \mathbf{v}_{\alpha, k}^{*} \overline{\mathbf{P}}^{*} \mathbf{H}_{\alpha, k}^{*} \mathbf{u}_{\alpha, k}}{\Delta_{\alpha, k}+\Gamma_{\alpha, k}+\Lambda_{\alpha, k}},
$$

where $\Delta_{\alpha, k}$ is the dominant intereference defined as

$$
\Delta_{\alpha, k}=\mathbf{u}_{\alpha, k}^{*} \sum_{\beta}\left(\frac{\rho_{\beta, k}}{S}\right) \mathbf{G}_{\beta, k} \overline{\mathbf{P}}\left[\mathbf{v}_{\beta, 1} \mathbf{v}_{\beta, 2} \ldots \mathbf{v}_{\beta, K}\right]\left[\begin{array}{llll}
\mathbf{v}_{\beta, 1} & \mathbf{v}_{\beta, 2} & \ldots & \mathbf{v}_{\beta, K}
\end{array}\right]^{*} \overline{\mathbf{P}}^{*} \mathbf{G}_{\beta, k}^{*} \mathbf{u}_{\alpha, k}
$$

$\Gamma_{\alpha, k}$ is the power of remaining interference and noise. Note that, channels of interferers generating remaining interference (denoted as $G_{\beta, k}^{r}$ ) are independent from those of dominant interferers. We take the expectation of the instantaneous remaining interference over channel matrices

$$
\mathrm{INR}_{\mathrm{rem}}=\mathbb{E}\left\{\operatorname{tr}\left(\sum_{\beta} \frac{\rho_{\beta, k}^{\mathrm{r}}}{S+\kappa^{2}} \mathbf{G}_{\beta, k}^{r} \overline{\mathbf{P}}\left[\begin{array}{cc}
\mathbf{I}_{S} & 0 \\
0 & \kappa^{2} \mathbf{I}_{N_{t}-S}
\end{array}\right] \overline{\mathbf{P}}^{*} \mathbf{G}_{\beta, k}^{r *}\right)\right\} .
$$


We assume that remaining interference is simply treated as noise with variance $\mathrm{INR}_{\text {rem. }}$. Then we calculate the average power of remaining interference and noise as

$$
\Gamma_{\alpha, k}=\mathbf{u}_{\alpha, k}^{*}\left(1+\mathrm{INR}_{\mathrm{rem}}\right) \quad \mathbf{u}_{\alpha, k}
$$

The power of intracell interference is given by

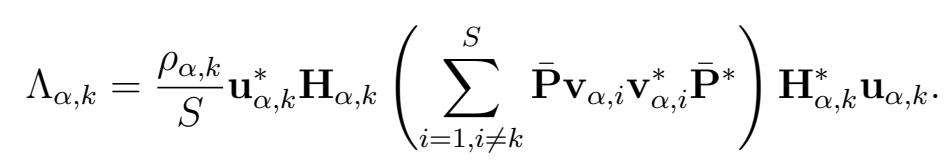

Substituting (5.48), (5.50) and (5.51) in (5.47), the corresponding ergodic rate per unit bandwidth for user $k$ is

$$
\mathrm{R}_{\alpha, k}=\mathbb{E}\left\{\log _{2}\left(1+\mathrm{SINR}_{\alpha, k}\right)\right\}
$$

and the ergodic sum rate per unit bandwidth for cell $\alpha$ is

$$
\tilde{\mathrm{R}}_{\alpha}=\sum_{k=1}^{S} \mathrm{R}_{\alpha, k}
$$

\subsubsection{Simulation Results}

In this section, we give simulation results. We first compare our proposed precoders with benchmark results of [3]. Then we investigate the impact of selecting the colouring parameter on the mean sum rates. Finally we give the simulation results of the cellular scenarios discussed in Chapter 2, with multiple dominant interferers, considering imperfect CSI and CCSI.

\section{Comparisons for Single Dominant Interferer Case - Benchmark Re- sults}

Fig. 5.6 presents simulation results to compare the SLNR and SLRINR-based precoders with the ZF based precoders of [3] for a multicell network, in terms of mean sum rates given in (5.53). Similar to [3], we assume that $N_{t}=N_{r}=4$ and there are $K=3$ in each cell. We also consider that the ratio of remaining interference over dominant interference is $\gamma=0.4$ on average as in [3], and only single dominant interferer is considered. In our simulations, we apply the MMSE-like receivers as in [3].

As seen in Fig. 5.6, SLNR-based precoders achieve better sum rates in low SNRs (noise-limited regions) and approximate to the ZF precoders as transmit SNR increases, because the intercell interference is also increasing. SLNR precoders achieve better sum rates than ZF precoders for transmit SNRs up to 15dB. Our proposed SLRINR based precoders, however, outperform ZF precoders for all 


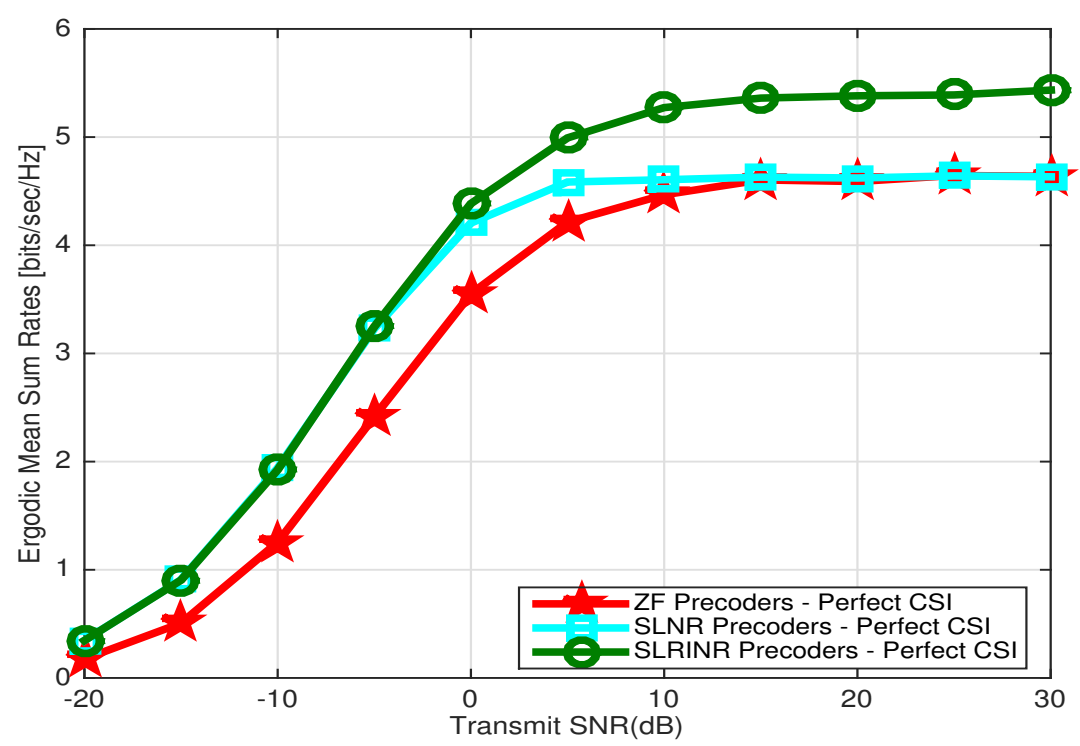

Figure 5.6: Comparison of the precoders with benchmark results of [12]. $\kappa$ is found from (5.25)

transmit SNRs. They provide a significant gain at high SNRs as well, because they minimise the effects of remaining interference which also increases with high transmit SNRs. The mean sum rates achieved with the SLRINR precoders is approximately $22 \%$ higher than with the $\mathrm{ZF}$ precoders at $30 \mathrm{~dB}$ transmit SNR.

\section{Selecting Colouring Parameter, $\kappa$}

In Fig. 5.7, we present simulation results of the mean sum rate using (5.53).

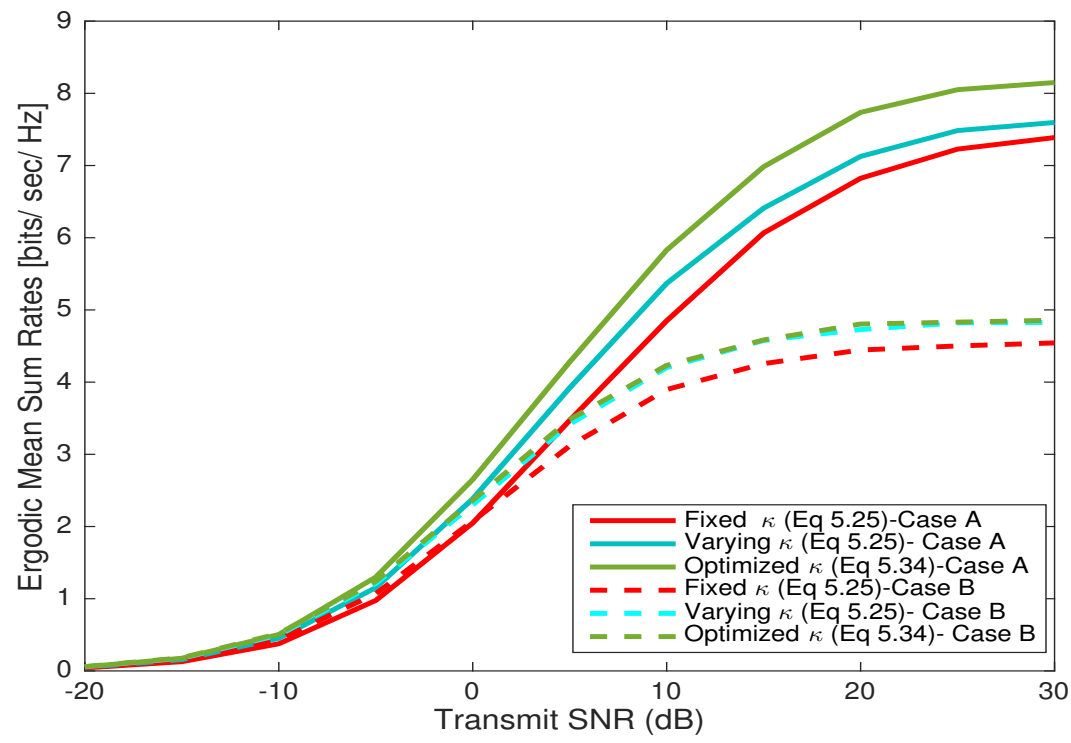

Figure 5.7: Comparison of optimised, variable and fixed $\kappa$ 
We also assume that each user has a different location, therefore different $\gamma$ ratios should be considered. We consider two cases. To be consistent with the simulation results given before for the optimization of $\kappa$, we define Case A where $\gamma_{1}=0.4, \gamma_{2}=0.1$ and $\gamma_{3}=0.01$. Previously, it is also mentioned that (5.25) is a good approximation for higher $\gamma$ ratios, therefore we also consider Case $B$ where we replace the lowest $\gamma$ ratio of case $\mathrm{A}, \gamma_{3}$, with a higher value, for which we arbitrarily choose $\gamma_{3}=0.8$. We compare these two cases corresponding to $\kappa$ values found from (5.25) and (5.34). For $\kappa$ value found by (5.25), we also compare fixed $\kappa$ for all users which was calculated for $\gamma=0.4$, and varying $\kappa$ for each user according to each of their $\gamma$ values.

Simulation results given in Fig. 5.7 indicate that for Case $A$, using varying $\kappa$ values from (5.25) for each user provides better sum rates than having a fixed $\kappa$ for all users. We also note that for Case A, optimizing $\kappa$ with (5.34) provides substantial gains. On the other hand, these gains vanish for higher gamma ratios as considered in Case $B$ where remaining interference is much stronger. We also note that using different $\kappa$ values for each user still achieves better sum rates than using a fixed $\kappa$ value for all users. However, this gain is not as significant as in Case A.

Comparison of the Precoders with Multiple Dominant Interferers, using Cellular Scenarios

We assume that there are $K=3$ users in each cell. We also assume that both transmitters and receivers are equipped with $N_{t}=N_{r}=4$ antennas respectively. We consider the 3GPP urban macro cell model in a 19-cell hexagonal network, using the COST 231 Hata urban path-loss model with log-normal shadowing as discussed in Chapter 2.

\section{- Scenario 1: Random User Locations}

We first evaluate simulations for random user locations. In Fig. 5.8, we give simulation results considering a single dominant interferer and 2 dominant interferers. From 19 cell simulations, we find $\gamma \approx 1.65$ on average if we assume a single dominant interferer, and $\gamma \approx 0.68$ on average if we assume 2 dominant interferers. Simulation results in Fig. 5.8 demostrate that SLNR precoders achieve better mean sum rates than the ZF precoders of [3] for lower transmit SNRs up to approximately $25 \mathrm{~dB}$. After this transmit SNR, the performance of SLNR precoders approximates that of ZF precoders. Overall, the proposed SLRINR-based precoders achieve better mean sum rates than the ZF precoders for all transmit SNRs. If we consider a single dominant interferer, the SLRINR-based precoders achieve mean sum rates $17 \%$ higher than ZF precoders. However, if we consider 2 dominant interferers, this difference decreases to $13 \%$. If we consider 2 dominant interferers, the level of unknown remaining interference decreases, therefore the gain which can be achieved by the use of the 


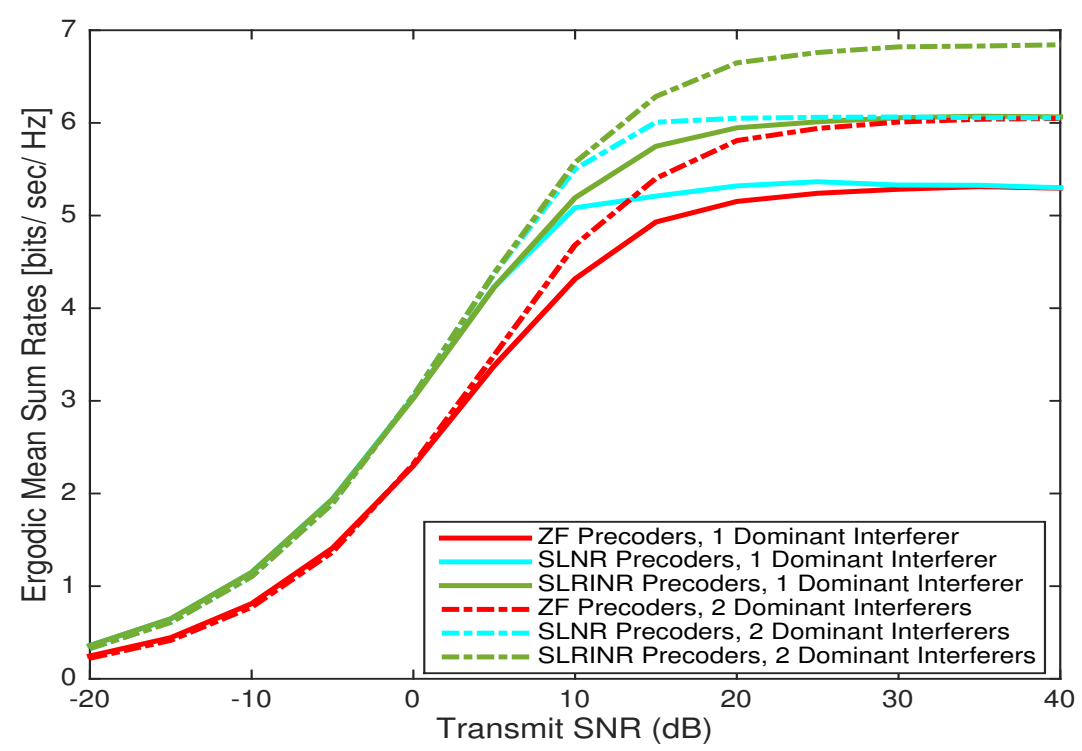

Figure 5.8: Ergodic mean sum rates with random locations (Scenario 1 - Downlink multicell systems )

SLRINR precoders decreases.

In Fig. 5.9, we demonstrate the performance of the precoders for 2 dominant interferers, considering imperfect CSI (between the desired BS and the users) and CCSI (between the interfering BSs and the desired users). First we consider imperfect CCSI which causes erroneous postcoders. The simulation results demonstrate that assuming $\varsigma=0.99$, erroneous postcoders do not cause a significant drop in mean sum rates. The reason is the fact that the postcoders cannot achieve a perfect cancellation of dominant interferers, even with perfect CCSI because the users do not know the actual precoders of the interfering BSs (See Eq. (5.27)). Hence, imperfect CCSI does not have a significant impact on the postcoders. Then we assume imperfect CSI which causes erroneous precoders and thus additional intracell interference. Assuming $\varsigma=0.99$, erroneous precoders cause a drastic degradation in mean sum rates. The ZF and SLNR precoders encounter a loss of $17 \%$ in mean sum rates whereas the SLRINR precoders lose approximately $11 \%$ of their mean sum rates at high transmit SNRs. However, we also note that SLRINR precoders with imperfect CSI still achieve better sum rates than the ZF precoders with perfect CSI. This shows us that the proposed SLRINR precoders are more robust to channel imperfections than the ZF and SLNR precoders. Then we also demonstrate the results with imperfect CSI and CCSI where all channel estimations are imperfect with $\varsigma=0.99$. The simulation results show that SLRINR precoders still achieve significantly better sum rates, approxi- 


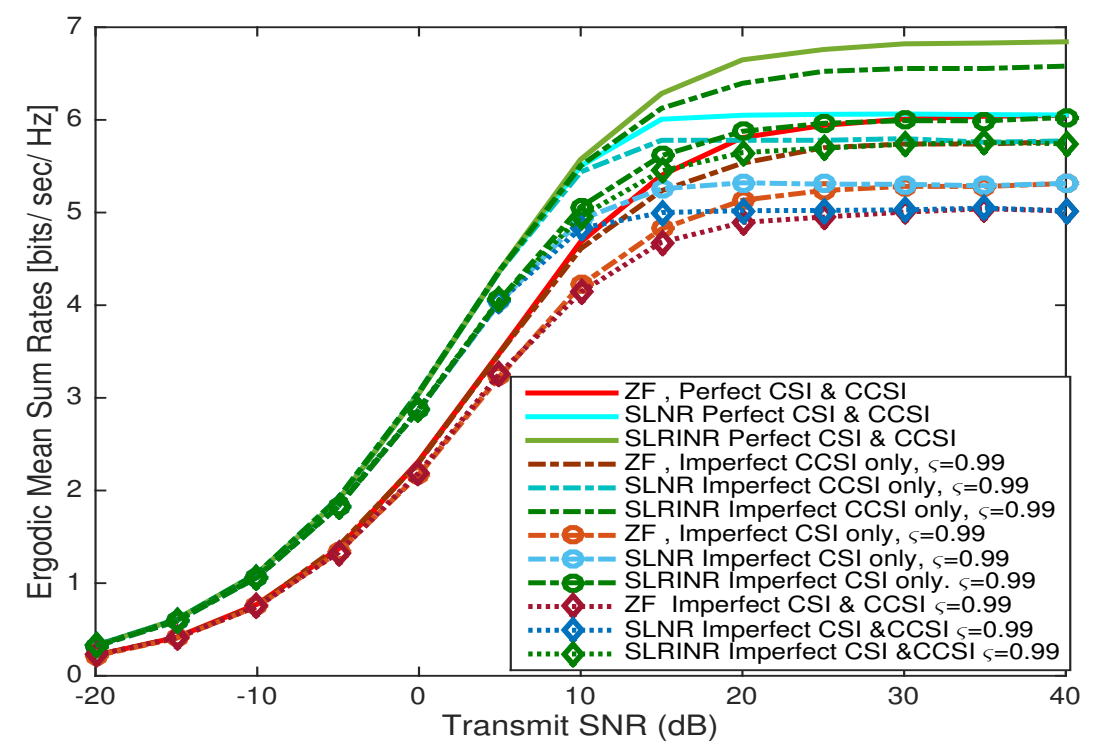

Figure 5.9: Ergodic mean sum rates for random locations with imperfect CSI and CCSI (Scenario 1 - Downlink multicell systems)

mately $16 \%$ more than the ZF and SLNR precoders with imperfect CSI and CCSI.

\section{- Scenario 2: Cell - Edge User Locations}

Here, we look at simulation results for cell-edge users only. We define the cell-edge users as given in Chapter 2. In Fig. 5.10, we demonstrate the gains that can be achieved considering 2 dominant interferers. These results indicate that cell-edge users encounter significant drop in mean sum rates. However, it is also shown that the SLRINR precoders provide significant gains compared to the ZF precoders. Considering a single dominant interferer, the SLRINR precoders can achieve approximately $57 \%$ higher mean sum rates than what the ZF precoders can achieve at high transmit SNR. If we consider 2 dominant interferers, the SLRINR precoders encounter approximately $13 \%$ increase in mean sum rates relative to single dominant interferer while the ZF and SLNR precoders encounter approximately $23 \%$ increase in mean sum rates relative to the single dominant interferer at $40 \mathrm{~dB}$ transmit SNR. However, the SLRINR precoders can achieve approximately $45 \%$ better mean sum rates than what the $\mathrm{ZF}$ and SLNR precoders can achieve with 2 dominant interferers at $40 \mathrm{~dB}$ transmit SNR.

Finally, in Fig. 5.11, we demonstrate the impact of the imperfect CSI and CCSI on the ergodic mean sum rates for cell-edge users, considering 2 dominant interferers. Imperfect CSI reduces the mean sum rates dramatically because of the additional intracell interference for cell-edge users. How- 


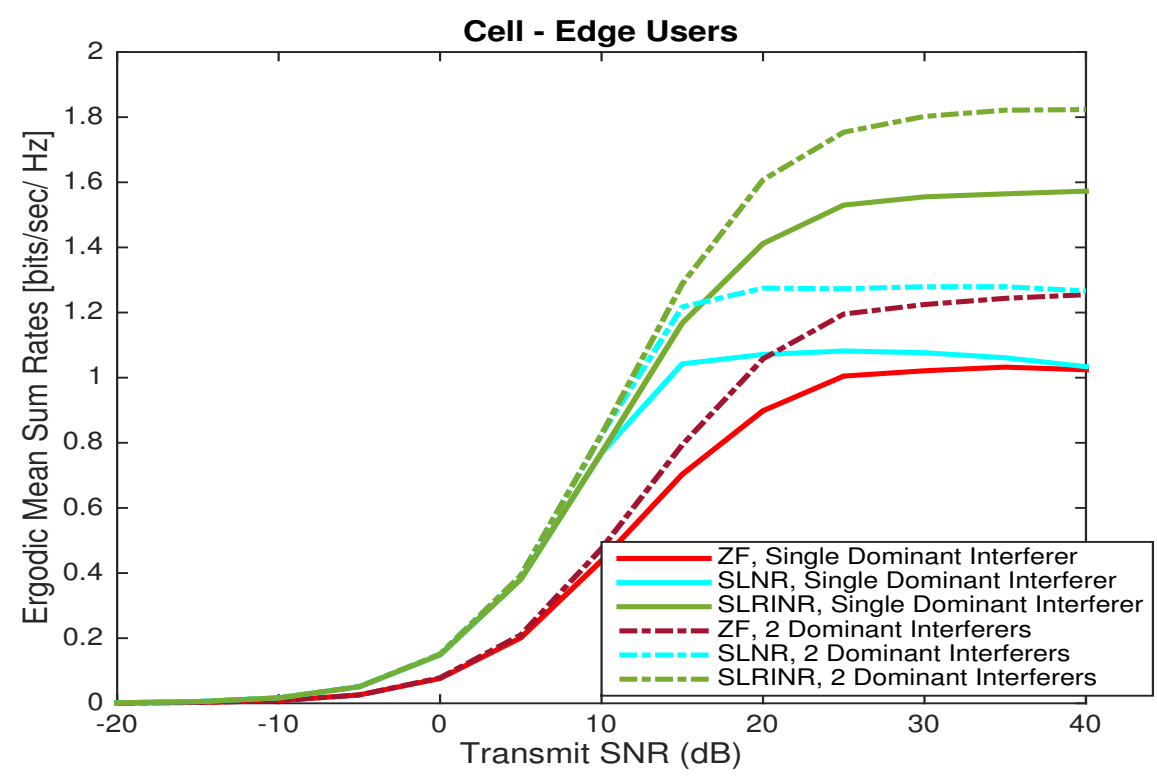

Figure 5.10: Ergodic mean sum rates for cell-edge locations (Scenario 2 - Downlink multicell systems)

ever, the simulation results indicate that the SLRINR precoders achieve better mean sum rates than other precoders with imperfect CSI and CCSI. Furthermore, it is also noted that the SLRINR precoders with imperfect CCSI $(\varsigma=0.99)$ achieve approximately $28 \%$ better mean sum rates than what ZF and SLNR precoders can achieve with perfect CCSI at $40 \mathrm{~dB}$ transmit SNR. These results show that our proposed SLRINR precoders are more robust to channel imperfections.

\subsection{Cooperation in Interference Alignment and Cancellation}

In this section, we investigate the impact of cooperation in an interference limited downlink cellular network [109]. We identify the areas in which the transmissions of adjacent cells overlap in a 19 cell network for urban and suburban environments. We assume that the cooperative users are located in these overlapping areas and thus can receive channel information from neighbouring BSs to cancel intercell interference. We also study the performance of such systems with various types of precoding techniques. We demonstrate that we can improve the system performance employing precoders that maximise SLNR and SLRINR for each user. We also consider imperfect CCSI between cooperative users and interfering BSs. Furthermore, we analytically derive the additional intercell interference that is caused by the imperfect CCSI at the receivers. Our results demonstrate that the system can achieve better sum rates with coopera- 


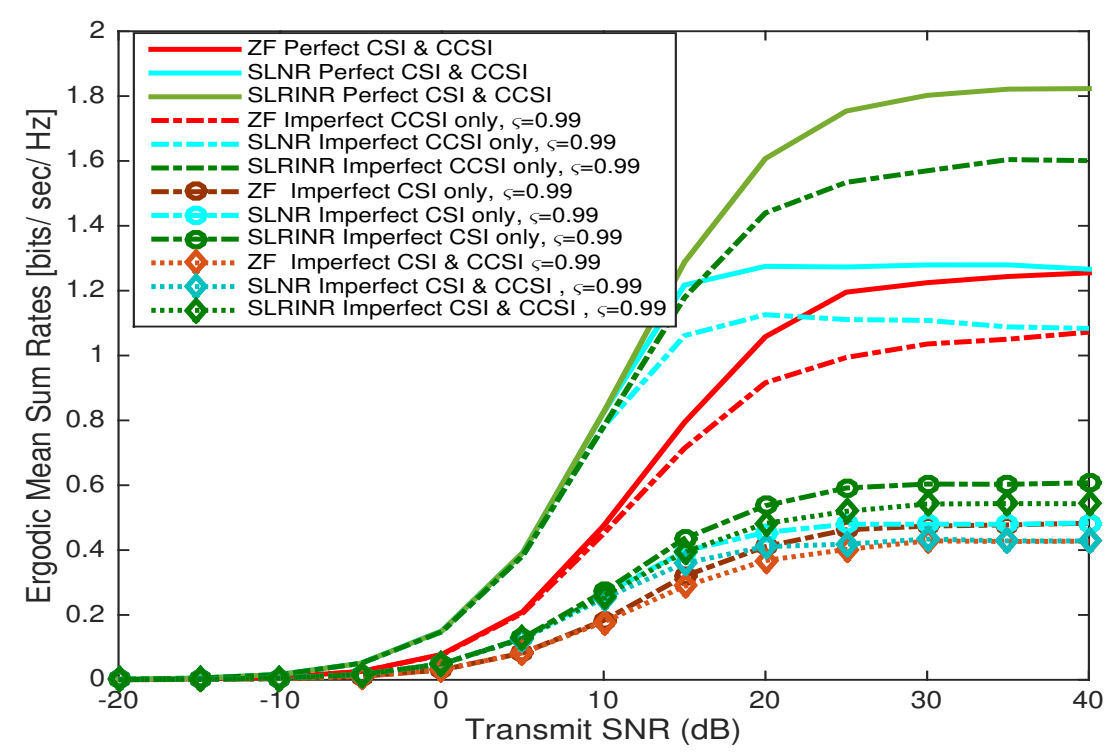

Figure 5.11: Ergodic mean sum rates for cell edge locations with Imperfect CSI and CCSI (Scenario 2 - Downlink multicell systems)

tion, however this improvement is susceptible to the accuracy of the CCSI. We show that imperfect CCSI degrades the cooperation gains drastically.

The contributions of this section are as follows

- We propose a partial cooperative interference cancellation scheme based on the received powers from adjacent BSs. This enables us to assess the merits of varying mixtures of cooperative and non-cooperative users and the gains achievable while reducing the overhead of the CCSI estimation between the interfering BS and the user.

- In order to show the impact of the environment type on the cooperation gains, we analyse our system for urban and suburban multicellular environments using the COST 231 Hata model. We demonstrate that the cooperation gains are different for different environments.

- We analytically derive expressions for the additional interference caused by the imperfect CCSI and demonstrate its impact on the receiver's performance in terms of mean sum rates and cooperation gains.

The received signal of user $i$ in cell $\alpha$ is given in (5.23) in the previous section. Similarly, the BS transmits its signals through 2 precoders: the secondary precoding matrix $\overline{\mathbf{P}}$ and $\mathbf{v}_{\alpha, i}$. The difference here is, the users activate different types of receivers depending on their locations. In the following section, we will give information on the receiver architecture of our proposed cooperative scheme. 


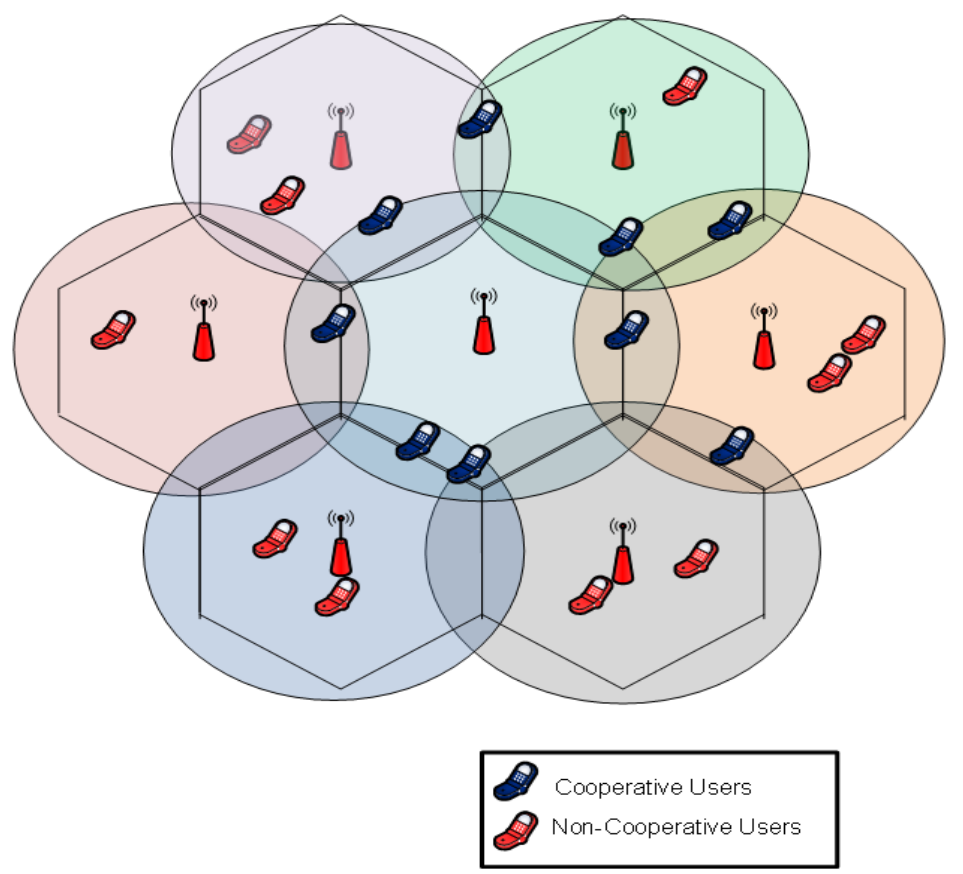

Figure 5.12: System model for cooperation in a downlink cellular network

\subsubsection{Receiver Architecture}

The receiver applies postcoding vectors, $\mathbf{u}_{\alpha, i}$, to the received signal to cancel intercell interference producing the received symbol

$$
\tilde{x}_{\alpha, i}=\mathbf{u}_{\alpha, i}^{*} \mathbf{y}_{\alpha, i}
$$

In [3], several receivers such as MF, MMSE-like and ZF receivers have been studied in the context of interference cancellation in multicellular networks. It has been shown in [3] that ZF receivers require perfect CCSI and sufficient antennas to null all interference. This is not practical when many interfering sources are present in the network. The MF receiver is preferred when there are many unknown interferers without any CCSI [3]. MMSE-like receivers are superior when the CCSI is available at the receivers [3]. Here, we assume that the users are equipped with both MF and MMSE-like receivers, and use either of them depending on whether they are located in the cooperation zone as in Fig 5.12.

\section{Non-cooperative Users}

Non-cooperative users utilise MF receivers due to the lack of CCSI. The postcoding vectors for MF receivers can be written as follows [3]

$$
\mathbf{u}_{\alpha, i}^{\mathrm{MF}}=\max \text { left singular vector of }\left(\mathbf{H}_{\alpha, i}\right) \text {. }
$$




\section{Cooperative Users}

The users located in the cooperation zone have CCSI and thus are able to employ the MMSE-like receivers which for user $i$ in cell $\alpha$ are given by [3]

$$
\mathbf{u}_{\alpha, i}^{\mathrm{MMSE}}=\left(\overline{\boldsymbol{\Phi}}_{\alpha, k}\right)^{-1} \mathbf{H}_{\alpha, k} \overline{\mathbf{P}}_{\mathbf{v}_{\alpha, k}}
$$

where the covariance matrix $\overline{\mathbf{\Phi}}_{\alpha, k}$ is given in (5.27). The postcoding vectors are also normalised to have a unit norm as in previous section, $\left\|\mathbf{u}_{\alpha, i}^{\mathrm{MME}}\right\|=1$

\subsubsection{Transmitter Architecture}

The BS generates the precoders, $\mathbf{v}_{\alpha, i}$, based on the equivalent channels $\mathbf{u}_{\alpha, i}^{*} \mathbf{H}_{\alpha, i} \overline{\mathbf{P}}$ fed back by the users. We assume a perfect feedback link. Using precoders, intracell interference can be mitigated without any information required at the receiver side. In this section, we consider ZF precoders, SLNR-based precoders and SLRINR-based precoders that are described in the previous section.

\subsubsection{The Algorithm}

We now describe the algorithm for the proposed cooperative interference cancellation scheme.

- Decision of cooperation The users decide on cooperation based on the ratio of channel strengths

$$
r_{\beta}=10 \log _{10}\left(\frac{\operatorname{tr}\left(\mathbf{H}_{\alpha, i} \mathbf{H}_{\alpha, i}^{*}\right)}{\operatorname{tr}\left(\mathbf{G}_{\beta, i} \mathbf{G}_{\beta, i}^{*}\right)}\right) .
$$

We set a threshold, $\Lambda$. If $r_{\beta}>\Lambda$, then the user is in a no-cooperation zone and it activates the MF receiver. If $r_{\beta}<\Lambda$, then the user is in a cooperation zone, receives CCSI from neighbouring BS(s), and activates the MMSE-like receiver. If all users are in the cooperation zone, we refer to this case as full cooperation. If some users are in the no-cooperation zone, then this is referred to as partial cooperation.

- Generating postcoding vectors Each user generates its postcoding vector according to its designation.

- Cooperative Users: MMSE postcoding vectors need precoders as given in (5.56). All precoders, ZF, SLNR and SLRINR-based precoding vectors also need postcoding vectors (See Eqs. (5.35) and (5.37)). 
Therefore, the cooperative users first initialize their postcoding vectors

$$
\mathbf{u}_{\alpha, i}^{(0)}=\left(\overline{\mathbf{\Phi}}_{\alpha, i}\right)^{-1} \mathbf{H}_{\alpha, i} \overline{\mathbf{P}}_{\mathbf{v}_{\alpha, i}^{(0)}}
$$

where we set $\mathbf{v}_{\alpha, i}^{(0)}$ as the maximum eigenvector of $\overline{\mathbf{P}}^{*} \mathbf{H}_{\alpha, i}^{*} \overline{\mathbf{\Phi}}_{\alpha, i}^{-1} \mathbf{H}_{\alpha, i} \overline{\mathbf{P}}$ [3].

- Non-cooperative Users: Non-cooperative users activate the MF receiver which does not need any initialization because the MF receiver doesn't require the precoding vectors. We generate non-cooperative users' postcoding vectors using (5.55).

- Generating Precoding Vectors Depending on the precoding type, the BS computes the precoding vectors.

\section{- ZF Precoders}

$$
\left[\begin{array}{llll}
\mathbf{v}_{\alpha, 1}^{(1)} & \mathbf{v}_{\alpha, 2}^{(1)} & \ldots & \mathbf{v}_{\alpha, k}^{(1)}
\end{array}\right]=\mathbf{H}^{(\mathbf{0})^{*}}\left(\mathbf{H}^{(\mathbf{0})} \mathbf{H}^{(\mathbf{0})^{*}}\right)^{-1}
$$

where $\mathbf{H}^{(0)}=\left[\mathbf{u}_{\alpha, 1}^{(0)^{*}} \mathbf{H}_{\alpha, 1} \overline{\mathbf{P}} ; \ldots ; \mathbf{u}_{\alpha, K}^{(0)^{*}} \mathbf{H}_{\alpha, K} \overline{\mathbf{P}}\right]$.

- SLNR Precoders $\mathbf{v}_{\alpha, i}^{(1)}$, is the leading eigenvector of

$$
\begin{aligned}
& \left(\mathbf{I}+\left(\frac{\rho_{\alpha, i}}{S}\right) \tilde{\mathbf{H}}_{\alpha, i}^{(0) *} \tilde{\mathbf{H}}_{\alpha, i}^{(0)}\right)^{-1} \overline{\mathbf{P}}^{*} \mathbf{H}_{\alpha, i}^{*} \mathbf{u}_{\alpha, i}^{(0)} \mathbf{u}_{\alpha, i}^{(0) *} \mathbf{H}_{\alpha, i} \overline{\mathbf{P}} \text {, where } \\
& \tilde{\mathbf{H}}_{\alpha, i}^{(0)}=\left[\begin{array}{llllll}
\mathbf{F}_{\alpha, 1}^{(0)^{T}} \mathbf{F}_{\alpha, 2}^{(0)^{T}} & \ldots & \mathbf{F}_{\alpha, i-1}^{(0)^{T}} \mathbf{F}_{\alpha, i+1}^{(0)^{T}} & \ldots & \mathbf{F}_{\alpha, K}^{(0)^{T}}
\end{array}\right]^{T} \\
& \text { and } \mathbf{F}_{\alpha, i}^{(0)}=\sqrt{\frac{\rho_{\alpha, i}}{S}} \mathbf{u}_{\alpha, i}^{(0)^{*}} \mathbf{H}_{\alpha, i} \overline{\mathbf{P}}
\end{aligned}
$$

- SLRINR Precoders $\mathbf{v}_{\alpha, i}^{(1)}$, is the leading eigenvector of

$$
\left(\left(1+\operatorname{INR}_{\mathrm{rem}}\right) \mathbf{I}+\left(\frac{\rho_{\alpha, i}}{S}\right) \tilde{\mathbf{H}}_{\alpha, i}^{(0) *} \tilde{\mathbf{H}}_{\alpha, i}^{(0)}\right)^{-1} \overline{\mathbf{P}}^{*} \mathbf{H}_{\alpha, i}^{*} \mathbf{u}_{\alpha, i}^{(0)} \mathbf{u}_{\alpha, i}^{(0) *} \mathbf{H}_{\alpha, i} \overline{\mathbf{P}}
$$

and then the BS use the precoders generated. In [3], iteration between the BS and the users was applied to update the postcoders and precoders. However, we don't use iteration in this work as it would increase the overhead dramatically. Finally we can summarise the system to give the precoders and postcoders for cooperative and non-cooperative users in the following table. 
Table 5.1: Summary of the system for a cooperative downlink cellular network

\begin{tabular}{|l|l|l|}
\hline & Precoder & Postcoder \\
\hline Cooperative Users & ZF/SLNR/SLRINR & MMSE-like \\
\hline Non-Cooperative Users & ZF/SLNR/SLRINR & MF \\
\hline
\end{tabular}

\subsubsection{Performance Metrics}

We consider 4 metrics to analyse the system performance: SINR, the cooperation gain, mean sum rates and the outage probability.

1 For the system described, the SINR for user $i$ in cell $\alpha$ is expressed in $(5.47)$

2 The cooperation gain is expressed as

$$
\phi=\mathbb{E}\left\{\frac{\operatorname{SINR}_{\alpha, i}^{\text {cooperation }}}{\operatorname{SINR}_{\alpha, i}^{\text {no-cooperation }}}\right\}
$$

$\operatorname{SINR}_{\alpha, i}^{\text {cooperation }}$ is the SINR achieved by the described cooperation scheme, while $\mathrm{SINR}_{\alpha, i}^{\text {no-cooperation }}$ represents the benchmark SINR where the system does not permit cooperation for any users.

3 The ergodic mean sum rate per unit bandwidth for cell $\alpha$ is given by

$$
\tilde{\mathrm{R}}_{\alpha}=\sum_{i=1}^{K} \mathbb{E}\left\{\log _{2}\left(1+\operatorname{SINR}_{\alpha, i}\right)\right\}
$$

4 Finally the outage probability for user $i$ in cell $\alpha$ is given by

$$
\mathrm{p}_{\alpha, i}^{\text {out }}\left(R_{\tau}\right)=\mathbb{P}\left\{\log _{2}\left(1+\operatorname{SINR}_{\alpha, i}\right)<R_{\tau}\right\}
$$

where $R_{\tau}$ is the chosen rate threshold. In [109], we assume that the users with received SINR less than $-5 \mathrm{~dB}$ are in outage [116], i.e., $R_{\tau}=\log _{2}(1+$ $\left.10^{-0.5}\right)=0.3964$.

\section{Instantaneous Intercell Interference with Imperfect CCSI}

Here we derive the instantaneous intercell interference with imperfect CCSI using (2.24). Therefore the additional instantaneous intercell interference can be estimated with respect to the channel imperfection parameter $\varsigma$. We first write the erroneous postcoding vectors for users in the overlapping regions by 
replacing $\mathbf{G}_{\beta, i}$ with $\hat{\mathbf{G}}_{\beta, i}$ in (5.56). This gives

$$
\hat{\mathbf{u}}_{\alpha, i}^{\mathrm{MMSE}}=\left(\hat{\boldsymbol{\Phi}}_{\alpha, i}\right)^{-1} \mathbf{H}_{\alpha, i} \overline{\mathbf{P}}_{\mathbf{v}_{\alpha, i}}
$$

where the erroneous covariance matrix is

$$
\begin{aligned}
\hat{\boldsymbol{\Phi}}_{\alpha, i} & =\left(1+\mathrm{INR}_{\mathrm{rem}}\right) \mathbf{I}_{N_{r}} \\
& +\sum_{\beta} \frac{\rho_{\beta, i}}{S+\left(N_{t}-S\right) \kappa^{2}} \hat{\mathbf{G}}_{\beta, i} \overline{\mathbf{P}}\left[\begin{array}{cc}
\mathbf{I}_{S} & \mathbf{0} \\
\mathbf{0} & \kappa^{2} \mathbf{I}_{N_{t}-S}
\end{array}\right] \overline{\mathbf{P}}^{*} \hat{\mathbf{G}}_{\beta, i}^{*} .
\end{aligned}
$$

Substituting (2.24) into (5.65), we obtain

$$
\begin{aligned}
& \hat{\mathbf{\Phi}}_{\alpha, i}=\left(1+\mathrm{INR}_{\mathrm{rem}}\right) \mathbf{I}_{N_{r}} \\
& +\sum_{\beta} \frac{\rho_{\beta, i}}{S+\left(N_{t}-S\right) \kappa^{2}}\left({ }_{\varsigma} \mathbf{G}_{\beta, i}+\bar{\varsigma} \boldsymbol{\Pi}_{\beta, i}\right) \overline{\mathbf{P}}\left[\begin{array}{cc}
\mathbf{I}_{S} & \mathbf{0} \\
\mathbf{0} & \kappa^{2} \mathbf{I}_{N_{t}-S}
\end{array}\right] \overline{\mathbf{P}}^{*}\left({ }_{\varsigma} \mathbf{G}_{\beta, i}+\bar{\varsigma}_{\beta} \boldsymbol{\Pi}_{\beta, i}\right)^{*} .
\end{aligned}
$$

We can rewrite (5.66) as

$$
\hat{\boldsymbol{\Phi}}_{\alpha, i}=\overline{\boldsymbol{\Phi}}_{\alpha, i}+\mathbf{T}_{\alpha, i}
$$

where

$$
\begin{aligned}
\mathbf{T}_{\alpha, i} & =\sum_{\beta} \frac{\rho_{\beta, i}}{S+\left(N_{t}-S\right) \kappa^{2}}\left(\left(\varsigma^{2}-1\right) \mathbf{G}_{\beta, i} \overline{\mathbf{P}}\left[\begin{array}{cc}
\mathbf{I}_{K} & \mathbf{0} \\
\mathbf{0} & \kappa^{2} \mathbf{I}_{N_{t}-K}
\end{array}\right] \overline{\mathbf{P}}^{*} \mathbf{G}_{\beta, i}^{*}\right) \\
& +\left(\varsigma \bar{\varsigma} \mathbf{G}_{\beta, i} \overline{\mathbf{P}}\left[\begin{array}{cc}
\mathbf{I}_{S} & \mathbf{0} \\
\mathbf{0} & \kappa^{2} \mathbf{I}_{N_{t}-S}
\end{array}\right] \overline{\mathbf{P}}^{*} \boldsymbol{\Pi}_{\beta, i}^{*}\right)+\left(\varsigma \bar{\varsigma} \mathbf{\Pi}_{\beta, i} \overline{\mathbf{P}}\left[\begin{array}{cc}
\mathbf{I}_{K} & \mathbf{0} \\
\mathbf{0} & \kappa^{2} \mathbf{I}_{N_{t}-K}
\end{array}\right] \overline{\mathbf{P}}^{*} \mathbf{G}_{\beta, i}^{*}\right) \\
& \left.+\left(\bar{\varsigma}^{2} \boldsymbol{\Pi}_{\beta, i} \overline{\mathbf{P}}\left[\begin{array}{cc}
\mathbf{I}_{K} & \mathbf{0} \\
\mathbf{0} & \kappa^{2} \mathbf{I}_{N_{t}-K}
\end{array}\right] \overline{\mathbf{P}}^{*} \boldsymbol{\Pi}_{\beta, i}^{*}\right)\right) .
\end{aligned}
$$

We drop the superscript of MMSE in (5.56) for notational clarity and write the intercell interference term as

$$
\hat{\Omega}_{\alpha, i}=\sum_{\beta}\left(\frac{\rho_{\beta, i}}{S}\right) \sum_{k=1}^{K} \hat{\mathbf{u}}_{\alpha, i}^{*} \mathbf{G}_{\beta, i} \overline{\mathbf{P}} \mathbf{v}_{\beta, k} \mathbf{v}_{\beta, k}^{*} \overline{\mathbf{P}}^{*} \mathbf{G}_{\beta, i}^{*} \hat{\mathbf{u}}_{\alpha, i}
$$


Substituting (5.56) and (6.9) into (5.69),

$$
\begin{aligned}
\hat{\Omega}_{\alpha, i} & =\left(\hat{\mathbf{\Phi}}_{\alpha, i}^{-1} \mathbf{H}_{\alpha, i} \overline{\mathbf{P}} \mathbf{v}_{\alpha, i}\right)^{*} \sum_{\beta}\left(\frac{\rho_{\beta, i}}{S}\right) \sum_{k=1}^{K} \mathbf{G}_{\beta, i} \overline{\mathbf{P}} \mathbf{v}_{\beta, k} \mathbf{v}_{\beta, k}^{*} \overline{\mathbf{P}}^{*} \mathbf{G}_{\beta, i}^{*}\left(\hat{\boldsymbol{\Phi}}_{\alpha, i}^{-1} \mathbf{H}_{\alpha, i} \mathbf{v}_{\alpha, i}\right) \\
& =\left(\left(\overline{\mathbf{\Phi}}_{\alpha, i}+\mathbf{T}_{\alpha, i}\right)^{-1} \mathbf{H}_{\alpha, i} \overline{\mathbf{P}}_{\mathbf{v}_{\alpha, i}}\right)^{*} \\
& \sum_{\beta}\left(\frac{\rho_{\beta, i}}{S}\right) \sum_{k=1}^{K} \mathbf{G}_{\beta, i} \overline{\mathbf{P}}_{\beta, k} \mathbf{v}_{\beta, k}^{*} \overline{\mathbf{P}}^{*} \mathbf{G}_{\beta, i}^{*}\left(\left(\overline{\mathbf{\Phi}}_{\alpha, i}+\mathbf{T}_{\alpha, i}\right)^{-1} \mathbf{H}_{\alpha, i} \overline{\mathbf{P}}_{\left.\mathbf{v}_{\alpha, i}\right)}\right.
\end{aligned}
$$

In order to find the instantaneous interference term with imperfect CCSI, we need to find the inverse of the erroneous covariance matrix $\hat{\boldsymbol{\Phi}}_{\alpha, i}^{-1}$ in (5.66) which involves the inverse of the summation of two full rank matrices, where the second matrix $\left(\mathbf{T}_{\alpha, i}\right)$ includes information about imperfect CCSI as given in (5.68). Taking the inverse of (5.66) is not straightforward. In [113], the authors provide a recursive solution for the inverse of a summation of two non-singular matrices which is applicable to our system. Following [113], we first decompose $\mathbf{T}_{\alpha, i}$ into matrices of rank 1. Therefore, let $\mathbf{T}_{\alpha, i}=\boldsymbol{\Upsilon}_{1}+\boldsymbol{\Upsilon}_{2}+\ldots+\boldsymbol{\Upsilon}_{\operatorname{rank}\left(\mathbf{T}_{\alpha, i}\right)}$. $\mathbf{\Upsilon}_{k}$ is simply a matrix in which the $k$ th column is the $k$ th column of $\mathbf{T}_{\alpha, i}$ and other elements are zero. Here we define $\mathbf{C}_{k+1}=\overline{\mathbf{\Phi}}_{\alpha, i}+\boldsymbol{\Upsilon}_{1}+\ldots+\boldsymbol{\Upsilon}_{k}$. Then if $\mathbf{C}_{1}=\overline{\mathbf{\Phi}}_{\alpha, i}$

$$
\hat{\boldsymbol{\Phi}}_{\alpha, i}^{-1}=\left(\overline{\boldsymbol{\Phi}}_{\alpha, i}+\mathbf{T}_{\alpha, i}\right)^{-1}=\overline{\mathbf{\Phi}}_{\alpha, i}^{-1}-\sum_{k=1}^{\operatorname{rank}\left(\mathbf{T}_{\alpha, i}\right)} \nu_{k} \mathbf{C}_{k}^{-1} \mathbf{\Upsilon}_{k} \mathbf{C}_{k}^{-1}
$$

where $\nu_{k}=\frac{1}{1+\operatorname{tr}\left(\mathbf{C}_{k}^{-1} \boldsymbol{\Upsilon}_{k}\right)}$.

Thus, the instantaneous intercell interference with imperfect CCSI is given by

$$
\begin{aligned}
& \hat{\Omega}_{\alpha, i}=\left(\left(\overline{\mathbf{\Phi}}_{\alpha, i}^{-1}-\sum_{k=1}^{\operatorname{rank}\left(\mathbf{T}_{\alpha, i}\right)} \nu_{k} \mathbf{C}_{k}^{-1} \mathbf{\Upsilon}_{k} \mathbf{C}_{k}^{-1}\right) \mathbf{H}_{\alpha, i} \overline{\mathbf{P}}_{\mathbf{v}_{\alpha, i}}\right)^{*} \\
& \sum_{\beta}\left(\frac{\rho_{\beta, i}}{S}\right) \sum_{k=1}^{K} \mathbf{G}_{\beta, i} \overline{\mathbf{P}}_{\mathbf{v}_{\beta, k} \mathbf{v}_{\beta, k}^{*}} \overline{\mathbf{P}}^{*} \mathbf{G}_{\beta, i}^{*}\left(\left(\overline{\mathbf{\Phi}}_{\alpha, i}^{-1}-\sum_{k=1}^{\operatorname{rank}\left(\mathbf{T}_{\alpha, i}\right)} \nu_{k} \mathbf{C}_{k}^{-1} \mathbf{\Upsilon}_{k} \mathbf{C}_{k}^{-1}\right) \mathbf{H}_{\alpha, i} \overline{\mathbf{P}} \mathbf{v}_{\alpha, i}\right) .
\end{aligned}
$$

We can represent the erroneous instantaneous intercell interference $\hat{\Omega}_{\alpha, i}$ with additional interference caused by the imperfect CCSI

$$
\hat{\Omega}_{\alpha, i}=\Omega_{\alpha, i}+\tilde{\Omega}_{\alpha, i}
$$

where $\tilde{\Omega}_{\alpha, i}$ is the additional interference caused by imperfect CCSI and is given 
as

$$
\begin{aligned}
& \tilde{\Omega}_{\alpha, i}=\left(\left(\sum_{k=1}^{\operatorname{rank}\left(\mathbf{T}_{\alpha, i}\right)} \nu_{k} \mathbf{C}_{k}^{-1} \mathbf{\Upsilon}_{k} \mathbf{C}_{k}^{-1}\right) \mathbf{H}_{\alpha, i} \overline{\mathbf{P}}_{\mathbf{v}_{\alpha, i}}\right)^{*} \mathbf{D}_{\alpha, i} \sum_{k=1}^{\operatorname{rank}\left(\mathbf{T}_{\alpha, i}\right)} \nu_{k} \mathbf{C}_{k}^{-1} \mathbf{\Upsilon}_{k} \mathbf{C}_{k}^{-1} \mathbf{H}_{\alpha, i} \overline{\mathbf{P}}_{\mathbf{v}_{\alpha, i}} \\
& -\left(\overline{\mathbf{\Phi}}_{\alpha, i}^{-1} \mathbf{H}_{\alpha, i} \overline{\mathbf{P}}_{\mathbf{v}_{\alpha, i}}\right)^{*} \mathbf{D}_{\alpha, i}\left(\left(\sum_{k=1}^{\operatorname{rank}\left(\mathbf{T}_{\alpha, i}\right)} \nu_{k} \mathbf{C}_{k}^{-1} \boldsymbol{\Upsilon}_{k} \mathbf{C}_{k}^{-1}\right) \mathbf{H}_{\alpha, i} \overline{\mathbf{P}}_{\mathbf{v}_{\alpha, i}}\right) \\
& -\left(\left(\sum_{k=1}^{\operatorname{rank}\left(\mathbf{T}_{\alpha, i}\right)} \nu_{k} \mathbf{C}_{k}^{-1} \boldsymbol{\Upsilon}_{k} \mathbf{C}_{k}^{-1}\right) \mathbf{H}_{\alpha, i} \overline{\mathbf{P}} \mathbf{v}_{\alpha, i}\right)^{*} \mathbf{D}_{\alpha, i}\left(\overline{\mathbf{\Phi}}_{\alpha, i}^{-1} \mathbf{H}_{\alpha, i} \overline{\mathbf{P}} \mathbf{v}_{\alpha, i}\right)
\end{aligned}
$$

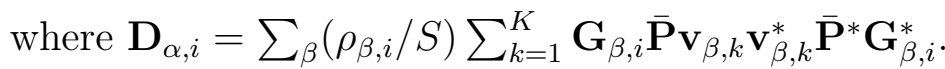

Main motivation to find (5.74) is to find the expected value of the additional interference. However, because (5.74) is such a complex expression, it is very hard to find the expected value. Therefore we only give the instantaneous additional interference as in (5.74).

\subsubsection{Simulation Results}

Here we give simulation results of our cooperative scheme. We consider $K=3$ users in each cell, equipped with $N_{r}=4$ antennas. We also assume that the BS is equipped with $N_{t}=4$ antennas.

\section{Urban Area Performance Comparisons}

We first evaluate the simulations for users in urban areas. We give mean sum rates using (5.62) for these users in Fig. 5.13. We compare the SLNR precoders with the ZF Precoders for no-cooperation, full cooperation and partial cooperation schemes. For partial cooperation, we assume $\Lambda=8 \mathrm{~dB}$ as mentioned in [97].

As seen in Fig. 5.13, the SLNR precoders provide better sum rates than the ZF precoders from very low transmit SNRs up to $25 \mathrm{~dB}$. After this value, the performance of the SLNR precoders approaches that of the ZF precoders. The simulation results also indicate that full cooperation can increase the mean sum rate by $55 \%$ compared to the no-cooperation scheme. On the other hand, setting $\Lambda=8 \mathrm{~dB}$, the partial cooperation scheme can increase the mean sum rate by $22 \%$ compared to the no-cooperation scheme.

In Fig. 5.14, assuming imperfect CCSI with a small error, $\varsigma=0.99$, the partial cooperation scheme can still increase the mean sum rate by $16 \%$ compared to the no-cooperation scheme. We also note here that SLRINR precoders provide significantly higher performance compared to other precoding schemes, however the gap between SLRINR precoders and other precoders decrease with increas- 
ing cooperation. The reason is that remaining interference is the main factor which makes SLRINR precoders better than the other precoders. As the remaining interference decreases with cooperation, the gap between SLRINR and other precoders also decrease.

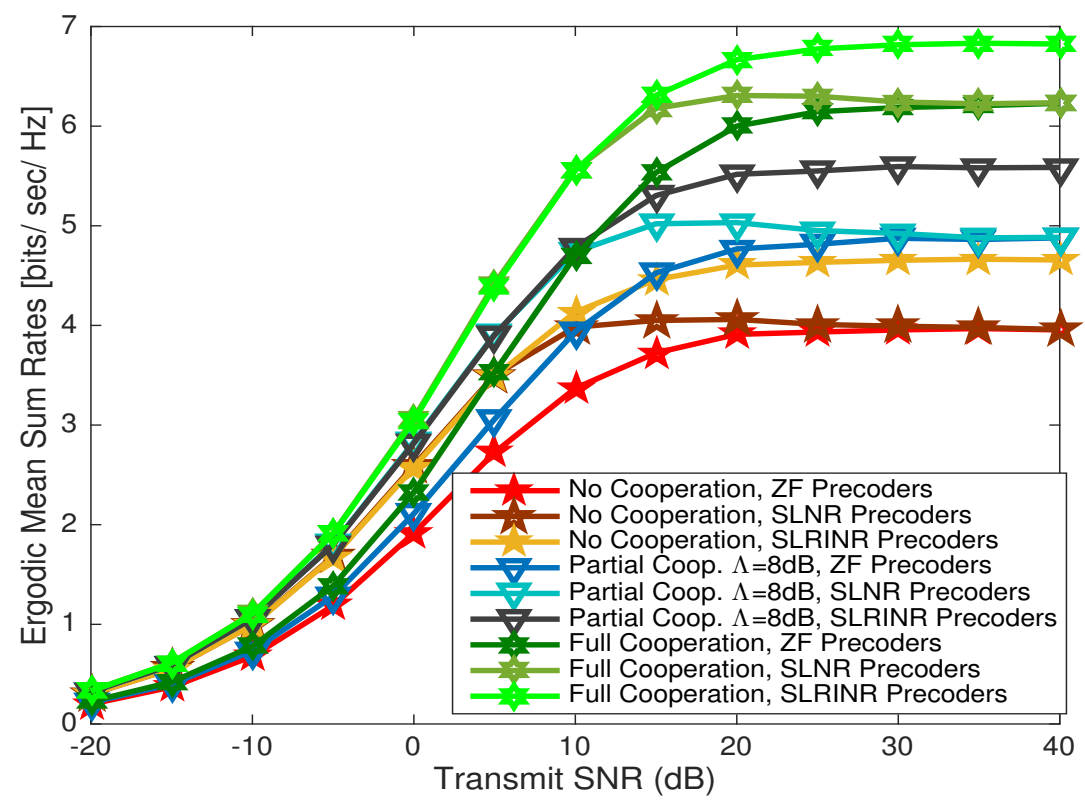

Figure 5.13: Ergodic mean sum rates for urban users with/without cooperation

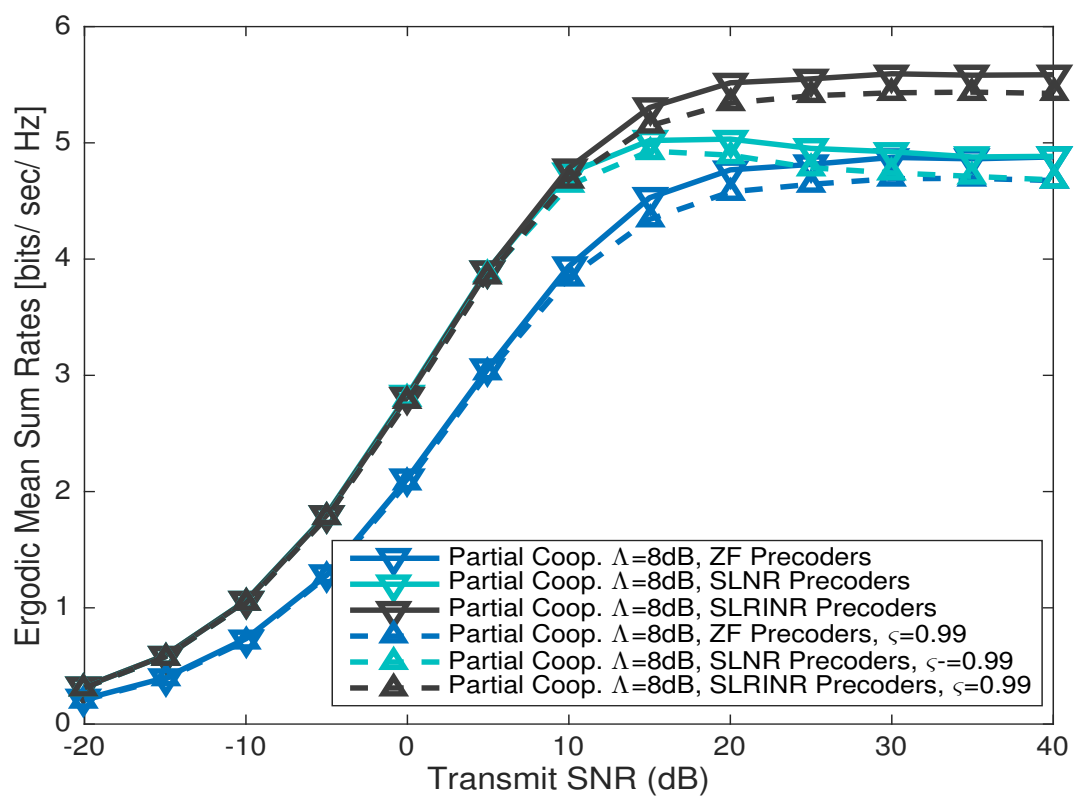

Figure 5.14: Ergodic mean sum rates for urban users with partial cooperation and imperfect CCSI

Next we find the cooperation gain defined as (5.61) with respect to the threshold, 
$\Lambda$ in Fig. 5.15. We assume the transmit SNR is $30 \mathrm{~dB} . \Lambda=0$ refers to nocooperation.

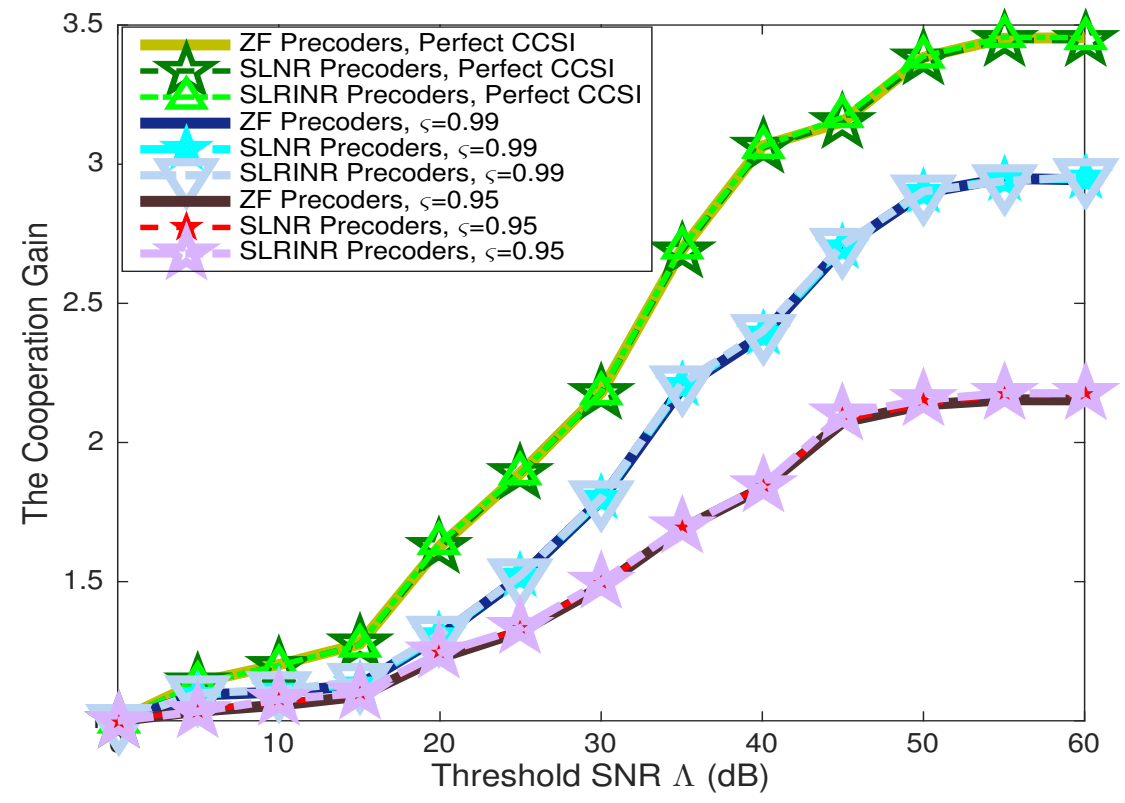

Figure 5.15: The cooperation gain for urban users

If we set the threshold in the region $\Lambda>50 \mathrm{~dB}$, the cooperation gain saturates as all users fall into the cooperation zone which is equivalent to the full cooperation scheme. The simulation results also demonstrate that imperfect CCSI significantly reduces the cooperation gain. For example, the full cooperation scheme encounters a very significant drop in cooperation gain with imperfect CCSI, from 3.48 to 2.94 and 2.19 for $\varsigma=0.99$ and $\varsigma=0.95$ respectively.

Table 5.2: Outage probability for urban users with/without cooperation

\begin{tabular}{|l|l|l|l|}
\hline \multicolumn{4}{|c|}{ Outage probability percentage (\%) } \\
\hline Precoders: & ZF & SLNR & SLRINR \\
\hline Full Cooperation & 13.6 & 13.3 & 8.7 \\
\hline Partial Cooperation & 14.5 & 13.9 & 9.5 \\
\hline Partial Cooperation $\varsigma=0.99$ & 16.1 & 15.3 & 11.0 \\
\hline No Cooperation & 28.3 & 27.7 & 17.5 \\
\hline
\end{tabular}

In Table 5.2, we give the outage probabilities for urban users at $30 \mathrm{~dB}$ transmit SNR. It can be seen that, the SLNR precoders outperform the ZF precoders by a small margin as expected, which is consistent with the results in Fig. 5.13, whereas the SLRINR precoders provide significant gains over the ZF and SLNR precoders. We also note that the outage probability with partial cooperation is significantly higher than no cooperation scheme as partial-cooperation improves 
the gains for cell-edge users in particular. The majority of the users which are below the chosen threshold are also located in the cooperation zone. Therefore, the outage probabilities with partial cooperation scheme are close to the outage probabilities with full cooperation scheme.

\section{Suburban Area Performance Comparisons}

Next, we evaluate the simulations for users in suburban areas. In Fig. 5.16, the mean sum rates (5.62) are given for suburban users. The results in Fig. 5.16 demonstrate that the SLNR precoders provide better sum rates than the ZF precoders from very low transmit SNRs up to $35 \mathrm{~dB}$, after which they provide approximately the same performace as ZF precoders. Here, we also observe that the performance of the SLNR precoders approaches the performance of the ZF precoders at higher transmit SNRs, compared to the urban users. The reason is the fact that the interference levels are lower for suburban users as seen in Chapter 2. We also note that SLRINR precoders achieve significantly higher mean sum rates, however this gain decreases if we increase the level of cooperation from no-cooperation to the full. We also observe in Fig. 5.17 that partial cooperation with imperfect CCSI where $\varsigma=0.99$ provides better mean sum rates compared to the no-cooperation scheme in Fig. 5.16.

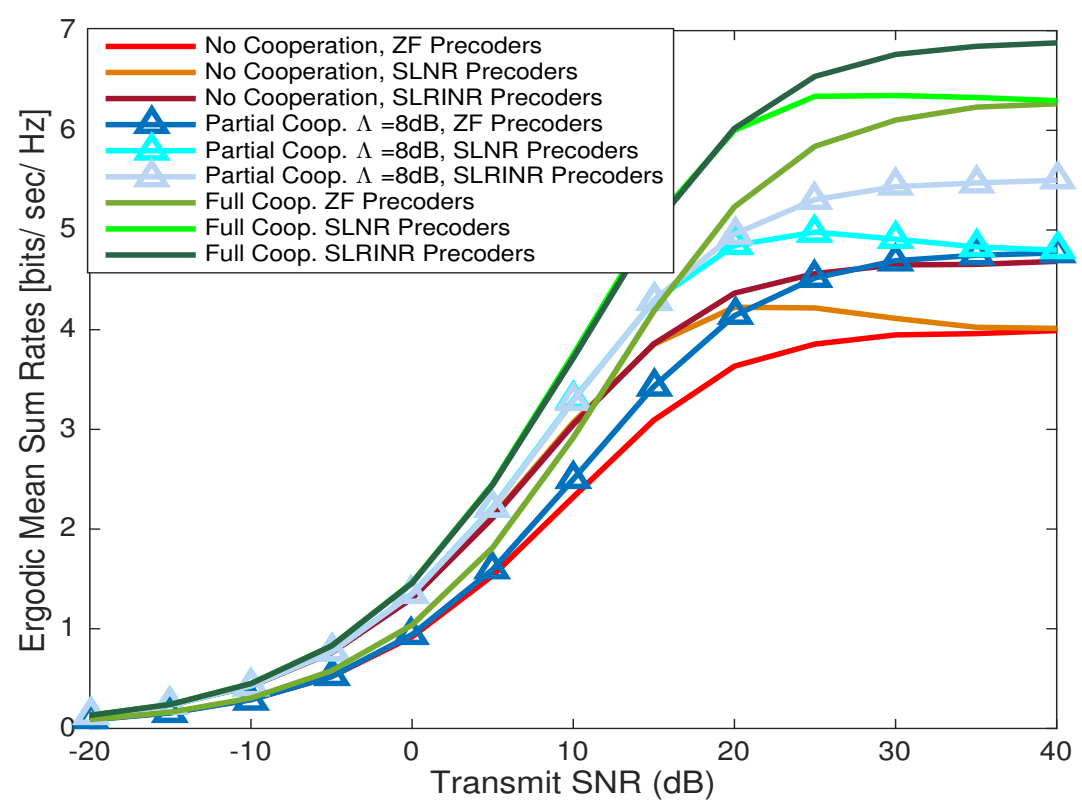

Figure 5.16: Ergodic mean sum rates for suburban users with/without cooperation 


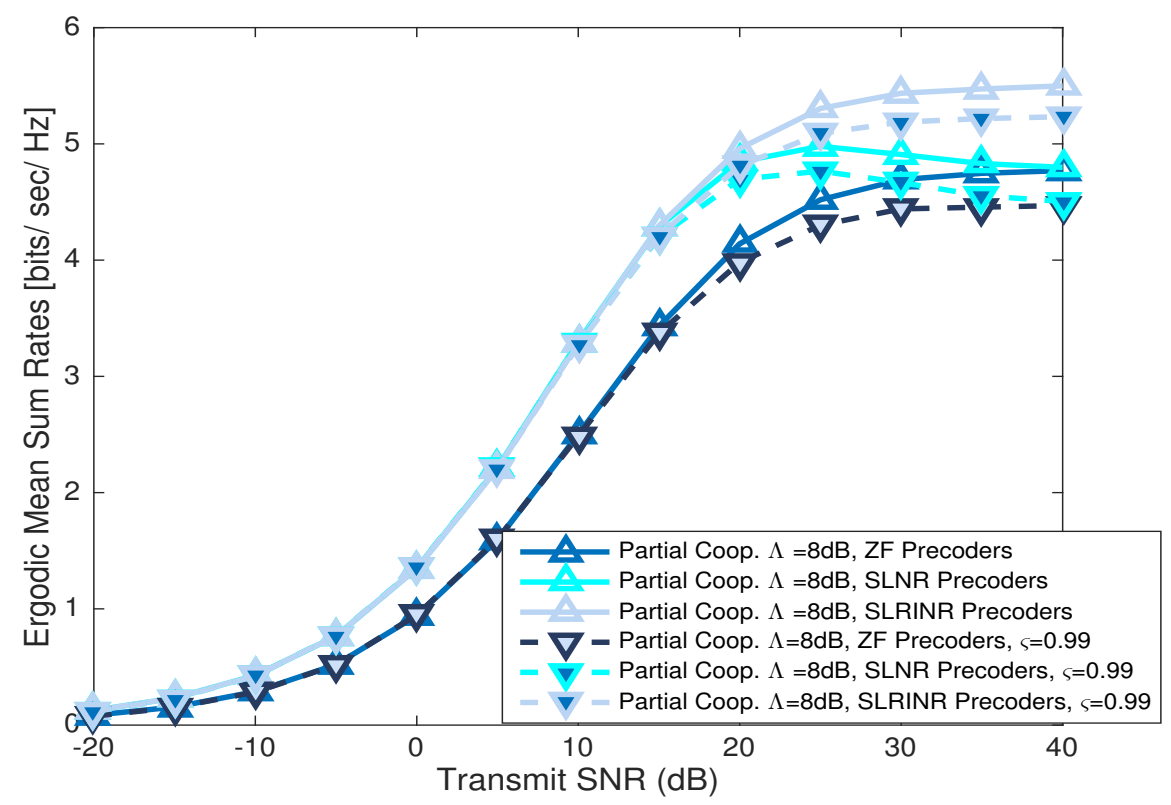

Figure 5.17: Ergodic mean sum rates for suburban users with partial cooperation and imperfect CCSI

In Fig. 5.18, we investigate the cooperation gain (5.61) vs threshold, $\Lambda$, for suburban users. We assume that the transmit $\mathrm{SNR}=30 \mathrm{~dB}$.

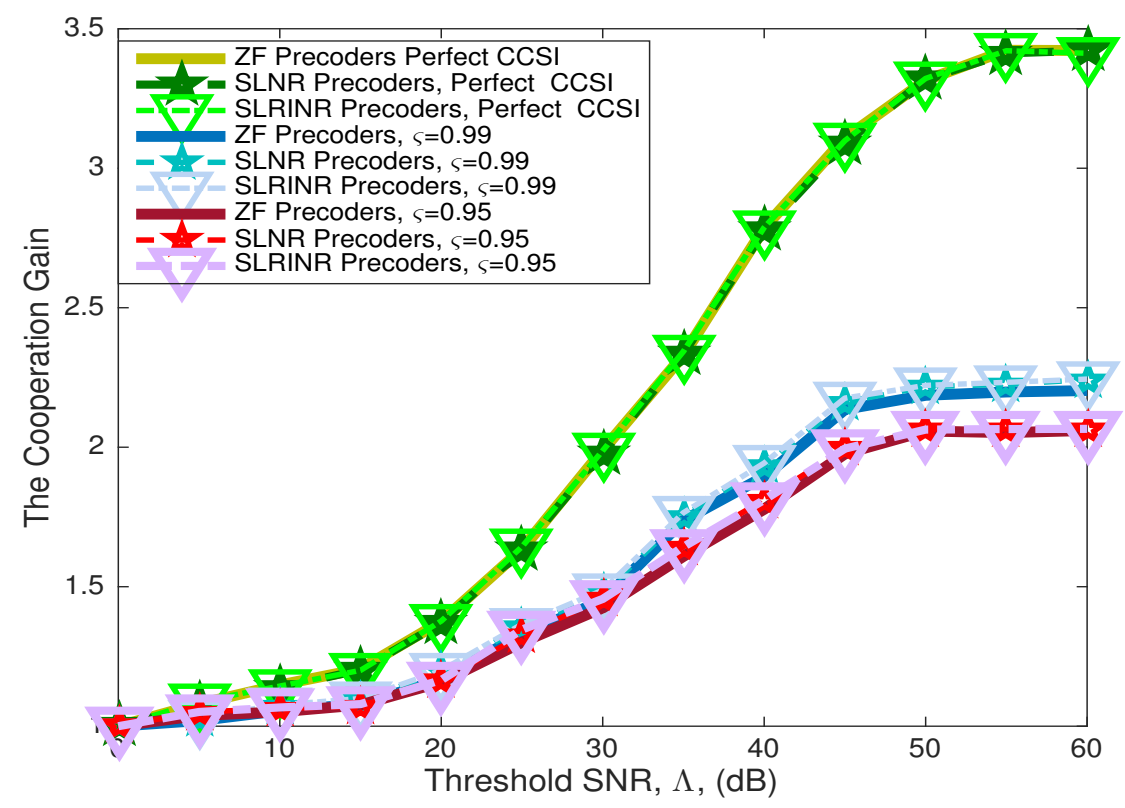

Figure 5.18: The cooperation gain for suburban users

As seen in Fig. 5.18, the full cooperation scheme suffers a drop in cooperation gain with imperfect CCSI, from 3.39 to 2.30 and 2.09 for $\varsigma=0.99$ and $\varsigma=$ 0.95 respectively. We note that the cooperation gains for suburban areas are 
lower than urban areas because interference for suburban areas is less than the interference in urban areas as shown in Chapter 2. However, suburban users suffer a loss in cooperation gain with imperfect CCSI more than urban area users, because the unknown remaining interference is also less for suburban areas as discussed in Chapter 2. The dominant interference, for which crosschannels are known and cancelled at the receiver, takes up more of the total interference for suburban area users. In other words, the $\gamma$ ratio is lower for suburban users. This explains why the imperfect CCSI impacts these users more than urban area users.

The outage probabilities for suburban users at transmit SNR 30dB are given in Table 5.3. Once again, the SLNR precoders perform better than the ZF precoders, which is consistent with Fig. 5.16. Furthermore, it is observed that the SLRINR precoders provide significant gains compared to the ZF and SLNR precoders. The results also indicate that partial cooperation provides significant gain compared to no cooperation scheme. We also observe that imperfect CCSI increases the outage probabilities significantly, and this increase is higher for suburban users than urban users. For example, for suburban users the outage probability increases from $9.82 \%$ to $14.83 \%$ with SLRINR precoders for suburban users with imperfect CCSI. However, for urban users employed with SLNR precoders, the imperfect CCSI increases the outage probability from $9.51 \%$ to $11.04 \%$, as given in Table 5.2 .

Table 5.3: Outage probability for suburban users with/without cooperation

\begin{tabular}{|l|l|l|l|}
\hline \multicolumn{4}{|c|}{ Outage probability percentage (\%) } \\
\hline Precoders: & ZF & SLNR & SLRINR \\
\hline Full Cooperation & 14.2 & 12.2 & 9.1 \\
\hline Partial Cooperation & 15.7 & 13.1 & 9.8 \\
\hline Partial Cooperation $\varsigma=0.99$ & 20.2 & 17.3 & 14.8 \\
\hline No Coopeartion & 29.8 & 27.1 & 17.7 \\
\hline
\end{tabular}

\section{Imperfect CCSI Analysis}

Finally, we investigate the accuracy of (5.74). We plot the CDF of the additional interference that is caused by the imperfect CCSI, assuming a transmit SNR at $30 \mathrm{~dB}$ and $\varsigma=0.99,0.95$ for urban and suburban users. 


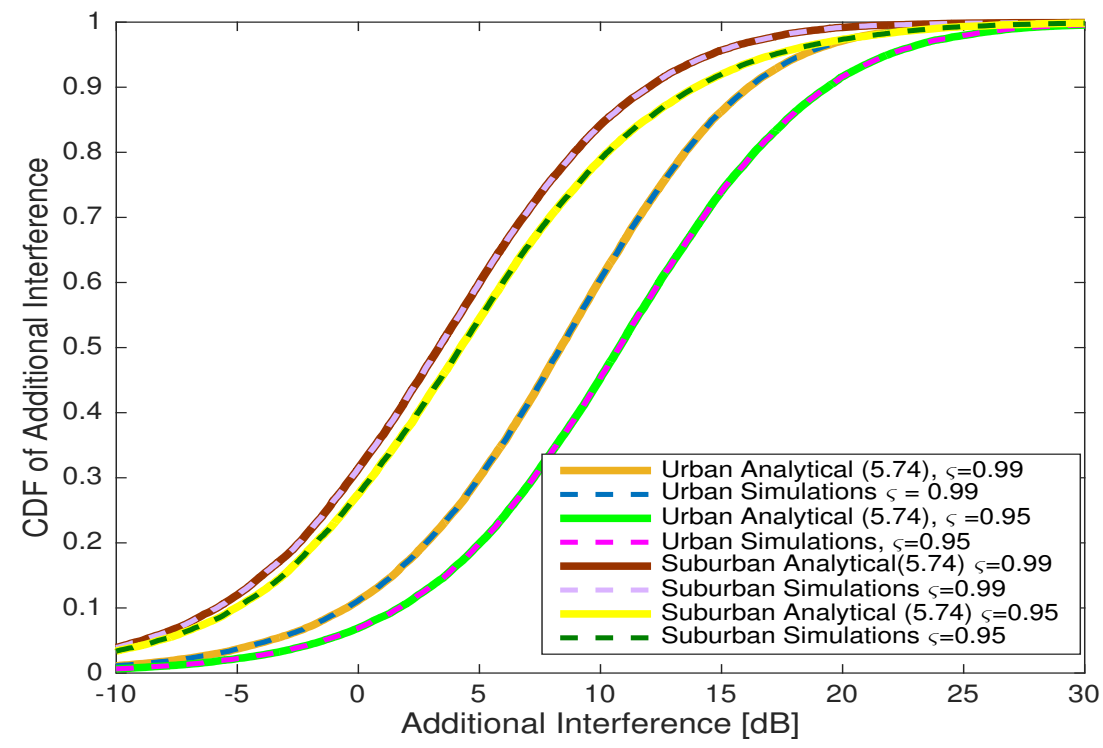

Figure 5.19: The CDF of additional interference that is caused by imperfect CCSI

In Fig. 5.19, it is observed that (5.74) is matching with the simulation results. Therefore we verify the accuracy of (5.74). As seen in this figure, the additional interference increases as $\varsigma$ decreases due to the reduced accuracy in the channel estimation. We also note that the additional interference for urban users is more than for suburban users as a result of path loss differences.

\subsection{Summary}

In this chapter, we have addressed how to apply IA schemes in downlink cellular networks. We have started by discussing the schemes with 2-cell case then we have shown how to deal with large networks where there are many cells serving many users.

First, we gave an IA scheme for a 2-cell downlink system in Section 4.2.1. We reproduced and discussed the results of [111]. We have also considered imperfect CSI for such systems and shown that IA for downlink systems is very susceptible to perfect channel estimation, similar to uplink systems.

Following the discussion for two cell downlink systems, we analysed a scheme that was proposed in [3] for cellular networks where there are more than two cells. We reproduced and expanded the results of [3] with their proposed MMSE-like postcoding vectors and ZF precoding vectors. We optimised the colouring parameter to improve the mean sum rates. Furthermore, we have applied SLNR-based precoding vectors which have been shown to perform better for low-transmit SNR regions and they achieve approximately same mean sum rates compared to ZF precoding vectors at high transmit SNR. We also 
proposed a new precoding vector based on SLRINR. As it considers the remaining interference, it provides significantly higher mean sum rates compared to other existing precoding schemes at all transmit SNRs. We also considered the imperfect CSI and showed that our proposed precoding scheme outperforms all other precoding schemes. We demonstrated that the proposed scheme provides significantly higher gain for cell-edge users as these users suffer from interference more than other users.

Finally in Section 4.3, we investigated a partial cooperative interference cancellation scheme for a downlink cellular network. We proposed that the cooperative users in the cooperation zone use MMSE-like receivers and can cancel intercell interference using CCSI from neighbouring cells. Other users located outside the cooperation zone have been assumed to use MF receivers. We showed that the system achieves better sum rates with cooperation, provided there is reasonable CCSI accuracy. Setting the cooperation threshold as $8 \mathrm{~dB}$, we have demonstrated that the partial cooperative scheme can achieve gains of approximately $25 \%$ in mean sum rates relative to the no-cooperation scheme at a transmit SNR of 40dB. We considered urban and suburban environments and computed the ratio of the users falling in the overlapping regions (i.e. subject to cooperation) for urban and suburban environments. We have also calculated the SNR, SINR levels for each of these environments. We compared the results for ZF, SLNR and SLRINR-based precoders and showed that the SLNR-based precoders achieve better sum rates at low transmit SNRs while their performance is equivalent to the ZF precoders at high transmit SNRs. However, we also showed that the transmit SNR value at which the performance of the SLNR precoders approaches that of the ZF precoders is different for different environments. For suburban users, this value is higher than for urban areas, because of lower interference levels. We also considered imperfect CCSI between the cooperative users and interfering BSs. What is more, we provided an analytical expression for additional interference caused by the imperfect CCSI. We confirmed the accuracy of this analytical expression via simulation results which show that analytical expression perfectly matches with the simulation results. We also noted that the additional interference for urban users is more than for suburban users as a result of path loss differences. 


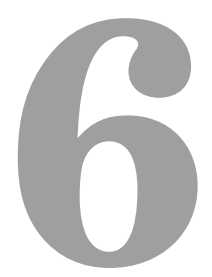

\section{Jointly Optimisation of Interference Alignment and Cancellation}

In this chapter, we focus on the jointly optimisation of the IAC techniques. We propose novel optimised transceivers which minimise total MSE for both the uplink and the downlink. For the uplink, we also investigate the optimisation using the combined receivers which we proposed in Chapter 4 . We analyse our system for randomly located users and cell-edge users. We investigate the performance loss with imperfect CSI and CCSI. The main contributions of this chapter are summarised as follows

- For the uplink, we propose two types of optimised transceivers minimising MSE for the desired cell. First, we consider optimising using an MMSE receiver of [85]. Later, we investigate optimising with the combined receiver of Section 4.2.3.

- For the downlink, we propose optimised transceivers using an MMSE receiver of Section 5.2. We do not consider optimisation with combined receivers, as it has been shown in Chapter 4 that the combined receivers are not suitable for downlink networks due to the antenna requirements at the receiver end.

- We analyse our systems considering cellular scenarios given in Chapter 2, comparing the techniques to existing schemes for both randomly located users and cell-edge users.

- We investigate the impact of imperfect channel estimation on the ergodic mean sum rates of each technique proposed in this chapter. 


\subsection{Jointly Optimisation of the Transmitters and the Receivers}

In Section 5.2.1, we proposed an IAC technique for a multicellular network in the downlink. The authors of [3] proposed a receiver which mimics MMSE receiver and showed that it outperforms ZF and MF techniques in systems where there are many interferers with different power levels. They introduced a colouring parameter, which is dependent on the interference levels that the users are exposed to and used to unify the extreme cases of these interference levels for receiver design. ZF precoders were used in [3] in order to cancel intracell interference, which we have shown in Section 5.2.1 that they provide poor performance when the remaining interference is high. Therefore we proposed the use of SLRINR precoders in Section 5.2.3.

In [3], the authors also used iteration to improve their system performance. However, this adds large complexity to the system as it requires feedback from transmitter to receiver for each iteration. Joint transmitter and receiver design can be an effective technique which can avoid undesired overhead and complexity in the system. It was shown that collective optimisation where the users adapt to optimise an overall system objective function together performs better than individual optimisation [117-119]. The authors of [85] proposed to jointly optimise transmitter and receiver sets for multiuser MIMO uplink systems. They worked with a system-wide measure to optimise the system: minimising the MSE. They showed that the minimising MSE function is jointly convex optimisation problem given the constraint set and the Karush-Kuhn-Tucker (KKT) conditions are satisfied. However, the authors of [85] did not extend their work for multicellular networks, nor did they address to the application of such systems in downlink networks.

In this chapter, we propose optimised transceivers for uplink and downlink multicellular networks. We propose to jointly optimise the transmitter and receiver set at the BS that minimises the total MSE. In the optimisation for downlink networks, we consider the MMSE receiver of Section 5.2.1 which is shown to be a very effective receiver technique where the system suffers from unknown interference (remaining interference). Because of the joint optimisation at the BS, our proposed system only needs one feedback link from the users to the $\mathrm{BS}$ in order to inform the users the optimum precoding vectors. That is why it does not need any iteration between the user and the BS as proposed in [3] (It does require iteration only in the BS). Furthermore, we also modify the scheme of [85] for uplink multicellular networks. In addition to this, for optimisation, we also consider the combined receiver of Section 4.2.3, which is shown to perform better when remaining interference is low. The 19-cell simulation results which have been given for uplink networks in Chapter 2 also support that remaining 
interference level for uplink networks is relatively low, therefore we investigate the application of the combined receiver in optimisation of such systems.

\subsection{Optimised Transceivers for Multicell Uplink Networks}

Consider the uplink multicellular network in Fig. 6.1. The main difference between Fig. 6.1 and Fig. 4.4 is the fact that there are only macro-cells in the network of Fig. 6.1. However, Fig. 4.4 is a mix of macro and pico cells. The system considered in this section also includes out-of-cell interference of which CSI is not known. The received signal at the $\mathrm{BS} \alpha$ is as follows

$$
\begin{aligned}
\mathbf{y}_{\alpha} & =\left(\sqrt{\frac{\rho_{\alpha, i}}{S}}\right) \mathbf{H}_{\alpha, i} \mathbf{v}_{\alpha, i} x_{\alpha, i}+\underbrace{\sum_{j=1, j \neq i}^{K}\left(\sqrt{\frac{\rho_{\alpha, j}}{S}}\right) \mathbf{H}_{\alpha, j} \mathbf{v}_{\alpha, j} x_{\alpha, j}}_{\text {Intracell Interference }} \\
& +\underbrace{\sum_{\beta} \sum_{l=1}^{K}\left(\sqrt{\frac{\rho_{\beta, l}}{S}}\right) \mathbf{G}_{\beta, l} \mathbf{v}_{\beta, l} x_{\beta, l}+\mathbf{z}_{\alpha}+\mathbf{n}_{\alpha},}_{\text {Dominant Intercell Interference }}
\end{aligned}
$$

where we define the terms for the $i$ th user in cell $\alpha . \mathbf{H}_{\alpha, i}$ is the desired channel, $\mathbf{G}_{\beta, i}$ is the dominant intercell interfering channel from adjacent cells, $\mathbf{v}_{\alpha, i}$ is the precoding vector, $x_{\alpha, i}$ is the transmitted signal, $\mathbf{z}_{\alpha}$ is the remaining interference and $\mathbf{n}_{\alpha}$ is the noise.

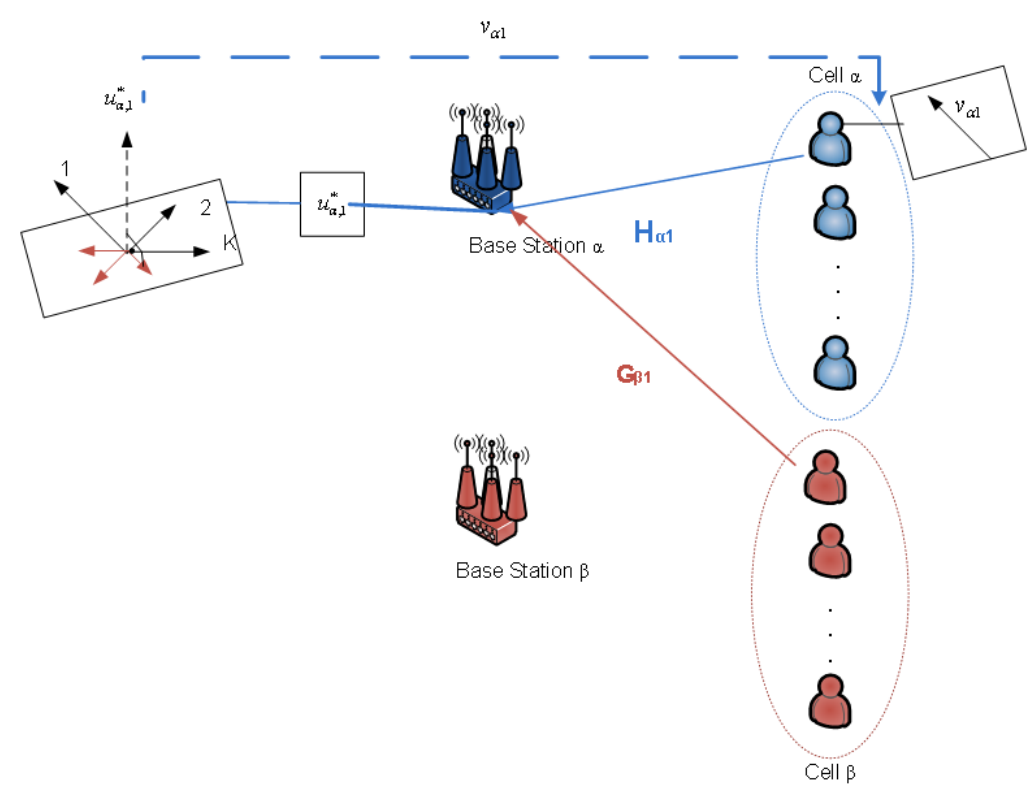

Figure 6.1: System model for multicell uplink networks 
We assume that there are $K$ users in each cell and $S$ streams are sent in total. As each user sends one stream, total number of streams is equal to number of users, $S=K$. Second term in (6.1) denotes the intracell interference when the BS decodes the signal of the $i$ th user. Note that $\beta$ denotes all dominant intercell interferers of which CCSI is available at the desired BS $\alpha$. As seen in Fig. 6.1, the BS generates the precoding vectors, informs the users. Then each user transmits desired signals using these precoding vectors.

The BS also generates the postcoder $\mathbf{u}_{\alpha, i}$ and multiplies the received signal $\mathbf{y}_{\alpha}$ with $\mathbf{u}_{\alpha, i}$ for each user to obtain the desired signal

$$
\tilde{x}_{\alpha, i}=\mathbf{u}_{\alpha, i}^{*} \mathbf{y}_{\alpha} .
$$

\subsubsection{The Algorithms of Optimised Transceivers For Mul- ticell Uplink Networks}

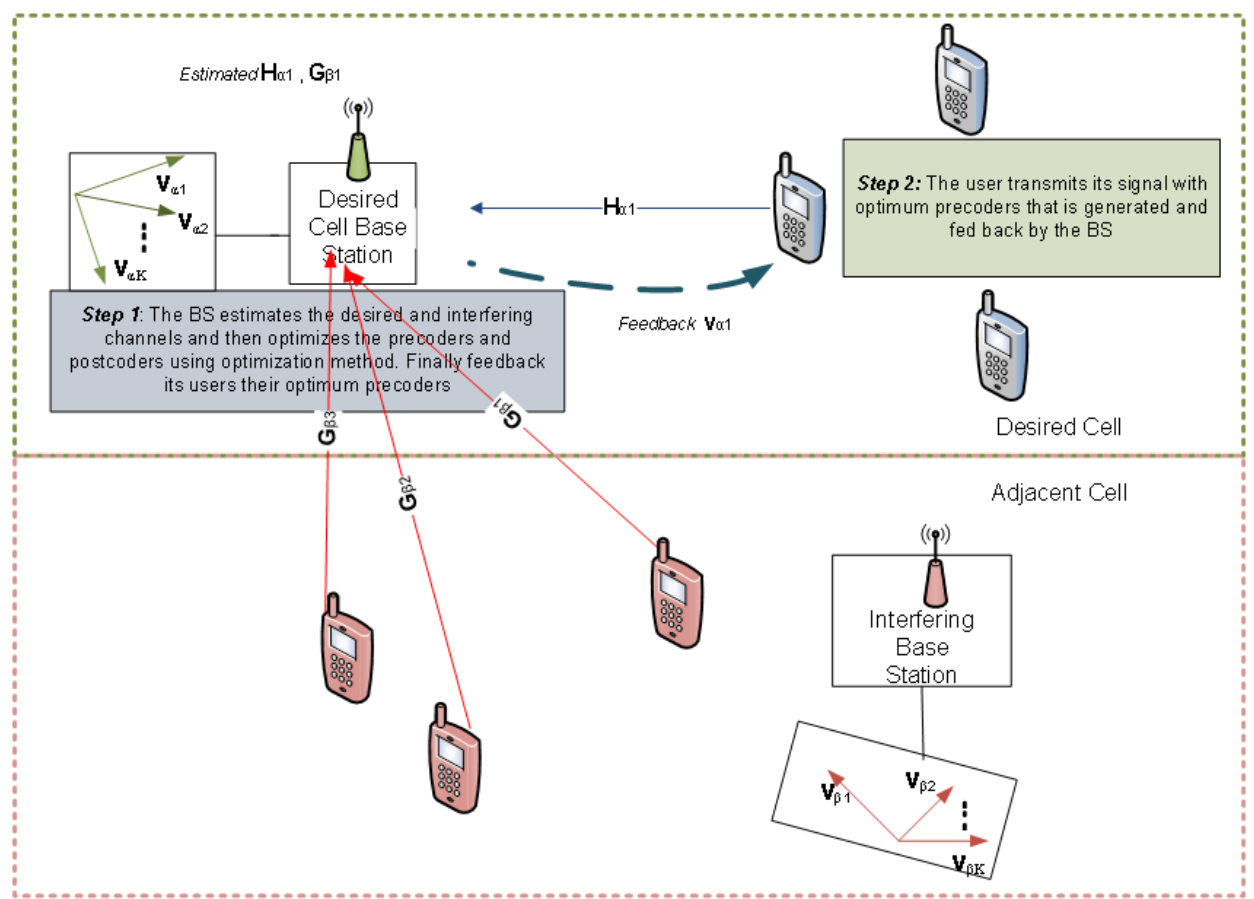

Figure 6.2: System model of the proposed optimised transceivers for uplink multicell networks

In this section, we expand the system in [85] to multicellular networks. We give the system model of the proposed optimised transceivers for the uplink in Fig. 6.2. We provide the algorithms for two types of optimised transceivers for uplink systems.

- Optimised Transceivers with MMSE Receivers: Here we recast the method of [85] for uplink multicellular networks using the MMSE Receiver of Chapter 4. 
- Optimised Transceivers with Combined Receivers: Here we recast the method of [85] using the receiver of Section 4.2 .3 which is referred to as the Combined Receiver.

\section{Optimised Transceivers with MMSE Receivers}

\section{The Algorithm}

The algorithm steps in order to find the optimum transceivers set for uplink systems using MMSE receivers are as follows

- Initialise the precoders and postcoders: The BS generates the initial precoding vectors, $\mathbf{v}_{\alpha, i}^{(0)}$, similar approach to [3],

$$
\begin{aligned}
\mathbf{v}_{\alpha, i}^{(0)} & =\max \text { eig. vector of }\left(\mathbf{H}_{\alpha, i}^{*} \overline{\boldsymbol{\Phi}}^{-1} \mathbf{H}_{\alpha, i}\right), \\
\text { such that } & :\left\|\mathbf{v}_{\alpha, \mathbf{i}}{ }^{(0)}\right\|=1,
\end{aligned}
$$

where $\overline{\boldsymbol{\Phi}}$ is given as

$$
\overline{\mathbf{\Phi}}=\left(1+\mathrm{INR}_{\mathrm{rem}}\right) \mathbf{I}+\underbrace{\left(\sum_{\beta} \sum_{j=1}^{K} \frac{\rho_{\beta, j}}{S} \mathbf{G}_{\beta, j} \mathbb{E}\left\{\mathbf{v}_{\beta, i} \mathbf{v}_{\beta, j}^{*}\right\} \mathbf{G}_{\beta, j}^{*}\right)}_{\text {Autocovariance matrix of dominant intercell interference }} .
$$

Since the out of cell precoders are not known in (6.4), we use $\mathbb{E}\left\{\mathbf{v}_{\beta, i} \mathbf{v}_{\beta, j}^{*}\right\}=$ $\frac{1}{N_{t}} \mathbf{I}$ which we have found via simulations.

Then the postcoder vector $\mathbf{u}_{\alpha, i}^{(0)}$ is generated using MMSE receivers [85],

$$
\mathbf{u}_{\alpha, i}^{(0)^{*}}=\mathbf{v}_{\alpha, i}^{(0) *} \mathbf{H}_{\alpha, i}^{*}\left(\sqrt{\frac{\rho_{\alpha, i}}{S}}\right) \mathbf{T}_{\alpha}^{(0)^{-1}}
$$

where

$$
\begin{aligned}
\mathbf{T}_{\alpha}^{(0)} & =\left(1+\mathrm{INR}_{\mathrm{rem}}\right) \mathbf{I}+\sum_{j=1}^{K} \frac{\rho_{\alpha, j}}{S} \mathbf{H}_{\alpha, j} \mathbf{v}_{\alpha, j}^{(0)} \mathbf{v}_{\alpha, j}^{(0) *} \mathbf{H}_{\alpha, j}^{*} \\
& +\left(\sum_{\beta} \sum_{j=1}^{K} \frac{\rho_{\beta, j}}{S} \mathbf{G}_{\beta, j}\left(\frac{1}{N_{t}} \mathbf{I}\right) \mathbf{G}_{\beta, j}^{*}\right) .
\end{aligned}
$$

The difference between the MMSE receiver we consider here and the receiver of [85] is the fact that we take into account the dominant and remaining intercell interference. 
- Optimisation: We find the set of optimum precoders and postcoders that minimise the total MSE. We compute MSE for each user following a similar way to [85], that is

$$
\operatorname{MSE}_{\alpha, i}^{(0)}=1-\mathbf{v}_{\alpha, i}^{(0) *} \mathbf{H}_{\alpha, i}^{*}\left(\frac{\rho_{\alpha, i}}{S}\right) \mathbf{T}_{\alpha, i}^{(0)^{-1}} \mathbf{H}_{\alpha, i} \mathbf{v}_{\alpha, i}^{(0)}
$$

Explanation of (6.7) is given in Appendix B. We then compute total $\mathrm{MSE}_{\alpha}$ for cell $\alpha$

$$
\operatorname{MSE}_{\alpha}^{(0)}=\sum_{i=1}^{K} \operatorname{MSE}_{\alpha, i}^{(0)}
$$

The inverse of $\mathbf{T}_{\alpha}^{(0)}$ can be found using matrix inversion lemma as given in [85]

$$
\mathbf{T}_{\alpha, i}^{(0)^{-1}}=\mathbf{E}_{\alpha, i}^{(0)^{-1}}-\frac{\mathbf{E}_{\alpha, i}^{(0)^{-1}} \mathbf{H}_{\alpha, i} \mathbf{v}_{\alpha, i}^{(0)}\left(\frac{\rho_{\alpha, i}}{S}\right) \mathbf{v}_{\alpha, i}^{(0) *} \mathbf{H}_{\alpha, i}^{*} \mathbf{E}_{\alpha, i}^{(0)^{-1}}}{1+\mathbf{v}_{\alpha, i}^{(0) *} \mathbf{H}_{\alpha, i}^{*}\left(\frac{\rho_{\alpha, i}}{S}\right) \mathbf{E}_{\alpha, i}^{(0)^{-1}} \mathbf{H}_{\alpha, i} \mathbf{v}_{\alpha, i}^{(0)}}
$$

where

$$
\begin{aligned}
\mathbf{E}_{\alpha, i}^{(0)} & =\left(\sum_{k=1, i \neq k}^{K} \frac{\rho_{\alpha, k}}{S} \mathbf{H}_{\alpha, k} \mathbf{v}_{\alpha, k}^{(0)} \mathbf{v}_{\alpha, k}^{(0) *} \mathbf{H}_{\alpha, k}^{*}\right) \\
& +\left(\sum_{\beta} \sum_{j=1}^{K} \frac{\rho_{\beta, j}}{S} \mathbf{G}_{\beta, j}\left(\frac{1}{N_{t}} \mathbf{I}\right) \mathbf{G}_{\beta, j}^{*}\right)+\left(1+\mathrm{INR}_{\mathrm{rem}}\right) \mathbf{I} .
\end{aligned}
$$

Then, we can rewrite (6.8) using (6.9) and (6.10) in (6.7) as given in [85]

$$
\begin{aligned}
\mathrm{MSE}_{\alpha}^{(0)} & = \\
& C_{i}-\left(1+\mathrm{INR}_{\mathrm{rem}}\right)\left(\frac{\mathbf{v}_{\alpha, i}^{(0) *} \mathbf{H}_{\alpha, i}^{*}\left(\frac{\rho_{\alpha, i}}{S}\right) \mathbf{E}_{\alpha, i}^{(0)^{-2}} \mathbf{H}_{\alpha, i} \mathbf{v}_{\alpha, i}^{(0)}}{\mathbf{v}_{\alpha, i}^{(0) *}\left(\mathbf{I}+\mathbf{H}_{\alpha, i}^{*}\left(\frac{\rho_{\alpha, i}}{S}\right) \mathbf{E}_{\alpha, i}^{(0)^{-1}} \mathbf{H}_{\alpha, i}\right) \mathbf{v}_{\alpha, i}^{(0)}}\right)
\end{aligned}
$$

where $C_{i}$ represents the term independent of the user $i$. We can find $C_{i}$ as $C_{i}=K-\operatorname{tr}\left(\mathbf{I}_{N_{r}}\right)+\left(1+\mathrm{INR}_{\mathrm{rem}}\right) \mathbf{E}_{\alpha, i}^{(0)^{-1}}$ following [85]. Note that $\mathbf{E}_{\alpha, i}^{(0)}$ does not depend on the precoding vector of the user $i, \mathbf{v}_{\alpha, i}$. From the perspective of the user $i$, MSE can be minimised by choosing $\mathbf{v}_{\alpha, i}$ to maximise the second term in (6.11), which is the term dependent on $\mathbf{v}_{\alpha, i}$. Using the Rayleigh Quotient Theorem [85,120], the precoding vectors that 
minimises MSE are given by

$$
\mathbf{v}_{\alpha, i}^{(1)}=\max \operatorname{eig} \operatorname{vec} .\left(\mathbf{H}_{\alpha, i}^{*} \frac{\rho_{\alpha, i}}{S} \mathbf{E}_{\alpha, i}^{(0)^{-2}} \mathbf{H}_{\alpha, i}, \quad \mathbf{I}+\mathbf{H}_{\alpha, i}^{*} \frac{\rho_{\alpha, i}}{S} \mathbf{E}_{\alpha, i}^{(0)^{-1}} \mathbf{H}_{\alpha, i}\right)
$$

subject to : $\left\|\mathbf{v}_{\alpha, i}^{(1)}\right\|=1$.

- Iteration: We update the $\mathbf{E}_{\alpha, i}$ at the $l$ th iteration using the updated precoding vectors

$$
\begin{aligned}
\mathbf{E}_{\alpha, i}^{(l)} & =\sum_{k=1}^{i-1} \frac{\rho_{\alpha, k}}{S} \mathbf{H}_{\alpha, k} \mathbf{v}_{\alpha, k}^{(l+1)} \mathbf{v}_{\alpha, k}^{(l+1) *} \mathbf{H}_{\alpha, k}^{*} \\
& +\sum_{j=i+1}^{K} \frac{\rho_{\alpha, j}}{S} \mathbf{H}_{\alpha, j} \mathbf{v}_{\alpha, j}^{(l)} \mathbf{v}_{\alpha, j}^{(l) *} \mathbf{H}_{\alpha, j}^{*} \\
& +\left(\sum_{\beta} \sum_{j=1}^{K} \frac{\rho_{\beta, j}}{S} \mathbf{G}_{\beta, j}\left(\frac{1}{N_{t}} \mathbf{I}\right) \mathbf{G}_{\beta, j}^{*}\right)+\left(1+\mathrm{INR}_{\mathrm{rem}}\right) \mathbf{I} .
\end{aligned}
$$

We update the precoders with updated $\mathbf{E}_{\alpha, i}^{(l)}$,

$$
\mathbf{v}_{\alpha, i}^{(l+1)}=\max \operatorname{eig} \operatorname{vec} .\left(\mathbf{H}_{\alpha, i}^{*}\left(\frac{\rho_{\alpha, i}}{S}\right) \mathbf{E}_{\alpha, i}^{(l)^{-2}} \mathbf{H}_{\alpha, i}, \quad \mathbf{I}+\mathbf{H}_{\alpha, i}^{*}\left(\frac{\rho_{\alpha, i}}{S}\right) \mathbf{E}_{\alpha, i}^{(l)^{-1}} \mathbf{H}_{\alpha, i}\right)
$$

subject to $:\left\|\mathbf{v}_{\alpha, i}^{(l+1)}\right\|=1$.

We keep iterating until $\epsilon=\mathrm{MSE}_{\alpha}^{(l+1)}-\mathrm{MSE}_{\alpha}^{(l)} \leq 0.001$. After finding the optimum precoding vectors, the BS generates the postcoders,

$$
\begin{aligned}
\mathbf{u}_{\alpha, i}^{(l+1)^{*}} & =\mathbf{v}_{\alpha, i}^{(l+1) *} \mathbf{H}_{\alpha, i}^{*}\left(\sqrt{\frac{\rho_{\alpha, i}}{S}}\right) \mathbf{T}_{\alpha, i}^{(l+1)^{-1}} \\
\text { such that } & : \quad\left\|\mathbf{u}_{\alpha, i}^{(l+1)}\right\|=1,
\end{aligned}
$$

where

$$
\begin{aligned}
\mathbf{T}_{\alpha}^{(l+1)} & =\left(1+\operatorname{INR}_{\mathrm{rem}}\right) \mathbf{I}+\left(\sum_{j=1}^{S} \frac{\rho_{\alpha, j}}{S} \mathbf{H}_{\alpha, j} \mathbf{v}_{\alpha, j}^{(l+1)} \mathbf{v}_{\alpha, j}^{(l+1) *} \mathbf{H}_{\alpha, j}^{*}\right) \\
& +\left(\sum_{\beta} \sum_{j=1}^{S} \frac{\rho_{\beta, j}}{S} \mathbf{G}_{\beta, j}\left(\frac{1}{N_{t}} \mathbf{I}\right) \mathbf{G}_{\beta, j}^{*}\right) .
\end{aligned}
$$

Finally, the BS feeds back the optimum precoders to the user in order to cancel the intracell interference. 


\section{Optimised Transceivers with Combined Receivers}

In the previous section, $\beta$ has denoted the dominant intercell interference of which the CCSI is available. In this section, we will specify the intercell interference which is significantly more than others and cancelled with ZF part of the combined receivers. These interferers are indexed as $\beta_{1} \in \beta$.

\section{The Algorithm}

Here, we modify the algorithm given in previous section, using the combined receivers.

- Initialise the precoders and postcoders: Similarly to optimised transceivers with MMSE receivers, we first generate the initial precoding vectors, $\mathbf{v}_{\alpha, i}^{(0)}$ as follows

$$
\begin{aligned}
\mathbf{v}_{\alpha, i}^{(0)} & =\max \text { eig. vector of }\left(\mathbf{H}_{\alpha, i}^{*} \overline{\mathbf{\Phi}}^{-1} \mathbf{H}_{\alpha, i}\right), \\
\text { such that } & :\left\|\mathbf{v}_{\alpha, \mathbf{i}}{ }^{(0)}\right\|=1,
\end{aligned}
$$

where $\overline{\mathbf{\Phi}}$ is given in (6.4).

Then, we write the postcoder vector $\mathbf{u}_{\alpha, i}^{(0)}$ using the combined receiver [1],

$$
\begin{aligned}
\mathbf{u}_{\alpha, i}^{(0)} & =\underbrace{\boldsymbol{\Xi}_{\alpha}}_{\mathrm{ZF} \text { part }} \underbrace{\mathbf{w}_{\alpha, i}^{(0)^{*}}}_{\text {MMSE part }} \\
\text { such that } & :\left\|\mathbf{u}_{\alpha, i}^{(0)}\right\|=1 .
\end{aligned}
$$

We find the ZF part of the receiver as given in Section 4.2.3. Please note that ZF part of the receiver is the same for all users in the same cell, as this is an uplink system, where intercell interference is same for all users. We first take the singular value decomposition of the dominant interference channel matrices (that will be cancelled using zero forcing) such that

$$
\begin{aligned}
\boldsymbol{\Psi}_{\alpha} & =\underbrace{\left[\mathbf{G}_{\beta_{1}, 1} \ldots \mathbf{G}_{\beta_{1}, K_{Z}}\right]}_{N r \times K_{Z} N_{t}} \\
& =\underbrace{\left[\boldsymbol{\Delta}_{\alpha}\right]}_{N_{r} \times N_{r}} \underbrace{\left[\mathbf{D}_{\alpha}\right]}_{N_{r} \times K_{Z} N_{t}} \underbrace{\left[\mathbf{V}_{\alpha}^{*}\right]}_{K_{Z} \times N_{t} \times K_{Z} N_{t}},
\end{aligned}
$$

where $\mathbf{G}_{\beta_{1}, k}$ denotes the channel from the dominant interfering user $k$ in adjacent cells to the BS of cell $\alpha, K_{Z}$ is the number of dominant interfering 
users to be nulled out with ZF and

$$
\mathbf{D}_{\alpha}=\left[\begin{array}{ccc}
\sqrt{\lambda_{1}} & 0 & \cdots \\
\vdots & \ddots & \vdots \\
0 & \cdots & \sqrt{\lambda_{K_{Z} N_{t}}} \\
0 & \cdots & 0 \\
\vdots & \ddots & \vdots \\
0 & \cdots & 0
\end{array}\right] .
$$

Here, the last $N_{r}-K_{Z} N_{t}$ rows of $\mathbf{D}_{\alpha}$ are zeros and $N_{r}-K_{Z} N_{t}$ must not be less than the number of interfering streams which is $K_{Z} N_{t}$. Hence the number of antennas at the receivers needs to satisfy $N_{r} \geq 2 K_{Z} N_{t}$.

We can denote $\boldsymbol{\Delta}_{\alpha}$ as

$$
\boldsymbol{\Delta}_{\alpha}=[\underbrace{\overline{\boldsymbol{\Delta}}_{a, l}}_{N_{r} \times K_{Z} N_{t}} \mid \underbrace{\boldsymbol{\Xi}_{\alpha}}_{N_{r} \times\left(N_{r}-K_{Z} N_{t}\right)}],
$$

where $\boldsymbol{\Xi}_{\alpha}$ is the ZF part of the receiver.

MMSE part of the receiver [1] which is denoted as $\mathbf{w}_{\alpha, i}$ in (6.18) aims to reduce the dominant intercell interference (of which power is not as significant as the dominant interferers indexed by $\beta_{1}$ ) with unknown remaining interference and also additive white Gaussian noise. The MMSE part of the combined receiver is found using [1]

$$
\mathbf{w}_{\alpha, i}^{(0)}=\mathbf{v}_{\alpha, i}^{(0) *} \mathbf{H}_{\alpha, i}^{*} \boldsymbol{\Xi}_{\alpha}\left(\sqrt{\frac{\rho_{\alpha, i}}{S}}\right) \hat{\mathbf{T}}_{\alpha}^{(0)^{-1}}
$$

where

$$
\begin{aligned}
\hat{\mathbf{T}}_{\alpha}^{(0)} & =\left(1+\mathrm{INR}_{\mathrm{rem}}\right) \mathbf{I}+\left(\sum_{j=1}^{K} \frac{\rho_{\alpha, j}}{S} \boldsymbol{\Xi}_{\alpha}^{*} \mathbf{H}_{\alpha, j} \overline{\mathbf{P}} \mathbf{v}_{\alpha, j}^{(0)} \mathbf{v}_{\alpha, j}^{(0) *} \overline{\mathbf{P}}^{*} \mathbf{H}_{\alpha, j}^{*} \boldsymbol{\Xi}_{\alpha}\right) \\
& +\left(\sum_{\beta \neq \beta_{1}} \sum_{j=1}^{K} \frac{\rho_{\beta, j}}{S} \boldsymbol{\Xi}_{\alpha}^{*} \mathbf{G}_{\beta, j}\left(\frac{1}{N_{t}} \mathbf{I}\right) \mathbf{G}_{\beta, j}^{*} \boldsymbol{\Xi}_{\alpha}\right) .
\end{aligned}
$$

- Optimisation: We compute the MSE for each user following the method given in [85], that is

$$
\operatorname{MSE}_{\alpha, i}^{(0)}=1-\mathbf{v}_{\alpha, i}^{(0) *} \mathbf{H}_{\alpha, i}^{*} \boldsymbol{\Xi}_{\alpha}\left(\frac{\rho_{\alpha, i}}{S}\right) \hat{\mathbf{T}}_{\alpha, i}^{(0)^{-1}} \boldsymbol{\Xi}_{\alpha}^{*} \mathbf{H}_{\alpha, i} \mathbf{v}_{\alpha, i}^{(0)}
$$


Then, we compute total $\mathrm{MSE}_{\alpha}$ for cell $\alpha$

$$
\operatorname{MSE}_{\alpha}^{(0)}=\sum_{i=1}^{K} \operatorname{MSE}_{\alpha, i}^{(0)}
$$

where $\operatorname{MSE}_{\alpha, 0}^{(0)}$ in (6.25) uses [85]

$$
\hat{\mathbf{T}}_{\alpha, i}^{(0)^{-1}}=\mathbf{E}_{\alpha, i}^{(0)^{-1}}-\frac{\mathbf{E}_{\alpha, i}^{(0)^{-1}} \boldsymbol{\Xi}_{\alpha}^{*} \mathbf{H}_{\alpha, i} \mathbf{v}_{\alpha, i}^{(0)}\left(\frac{\rho_{\alpha, i}}{S}\right) \mathbf{v}_{\alpha, i}^{(0) *} \mathbf{H}_{\alpha, i}^{*} \boldsymbol{\Xi}_{\alpha} \mathbf{E}_{\alpha, i}^{(0)^{-1}}}{1+\mathbf{v}_{\alpha, i}^{(0) *} \mathbf{H}_{\alpha, i}^{*} \boldsymbol{\Xi}_{\alpha}\left(\frac{\rho_{\alpha, i}}{S}\right) \mathbf{E}_{\alpha, i}^{(0)^{-1}} \boldsymbol{\Xi}_{\alpha}^{*} \mathbf{H}_{\alpha, i} \mathbf{v}_{\alpha, i}^{(0)}}
$$

with

$$
\begin{aligned}
\mathbf{E}_{\alpha, i}^{(0)} & =\left(\sum_{k=1, i \neq k}^{K} \frac{\rho_{\alpha, k}}{S} \boldsymbol{\Xi}_{\alpha}^{*} \mathbf{H}_{\alpha, k} \mathbf{v}_{\alpha, k}^{(0)} \mathbf{v}_{\alpha, k}^{(0) *} \mathbf{H}_{\alpha, k}^{*} \boldsymbol{\Xi}_{\alpha}\right) \\
& +\left(\sum_{\beta \neq \beta_{1}} \sum_{j=1}^{K} \frac{\rho_{\beta, j}}{S} \boldsymbol{\Xi}_{\alpha}^{*} \mathbf{G}_{\beta, j}\left(\frac{1}{N_{t}} \mathbf{I}\right) \mathbf{G}_{\beta, j}^{*} \boldsymbol{\Xi}_{\alpha}\right)+\left(1+\mathrm{INR}_{\mathrm{rem}}\right) \mathbf{I} .
\end{aligned}
$$

We can rewrite (6.26) substituting (6.27) and (6.28) in (6.25), giving

$$
\operatorname{MSE}_{\alpha}^{(0)}=C_{i}-\left(1+\operatorname{INR}_{\mathrm{rem}}\right)\left(\frac{\mathbf{v}_{\alpha, i}^{(0) *} \mathbf{H}_{\alpha, i}^{*} \boldsymbol{\Xi}_{\alpha}\left(\frac{\rho_{\alpha, i}}{S}\right) \mathbf{E}_{\alpha, i}^{(0)^{-2}} \boldsymbol{\Xi}_{\alpha}^{*} \mathbf{H}_{\alpha, i} \mathbf{v}_{\alpha, i}^{(0)}}{\mathbf{v}_{\alpha, i}^{(0) *}\left(\mathbf{I}+\mathbf{H}_{\alpha, i}^{*} \boldsymbol{\Xi}_{\alpha}\left(\frac{\rho_{\alpha, i}}{S}\right) \mathbf{E}_{\alpha, i}^{(0)^{-1}} \boldsymbol{\Xi}_{\alpha}^{*} \mathbf{H}_{\alpha, i}\right) \mathbf{v}_{\alpha, i}^{(0)}}\right),
$$

where $C_{i}$ represents the term independent of user $i$. We can modify (6.12) for optimised transceivers with combined receivers, giving

$$
\begin{aligned}
& \mathbf{v}_{\alpha, i}^{(1)}=\max \text { eig vec. } \\
& \left(\mathbf{H}_{\alpha, i}^{*} \boldsymbol{\Xi}_{\alpha} \frac{\rho_{\alpha, i}}{S} \mathbf{E}_{\alpha, i}^{(0)^{-2}} \boldsymbol{\Xi}_{\alpha}^{*} \mathbf{H}_{\alpha, i}, \quad \mathbf{I}+\mathbf{H}_{\alpha, i}^{*} \boldsymbol{\Xi}_{\alpha} \frac{\rho_{\alpha, i}}{S} \mathbf{E}_{\alpha, i}^{(0)^{-1}} \boldsymbol{\Xi}_{\alpha}^{*} \mathbf{H}_{\alpha, i}\right) \\
& \text { subject to : }\left\|\mathbf{v}_{\alpha, i}^{(1)}\right\|=1 .
\end{aligned}
$$

- Iteration: We can refine $\mathbf{E}_{\alpha, i}$ at $l$ th iteration using the updated precoding 
vectors,

$$
\begin{aligned}
\mathbf{E}_{\alpha, i}^{(l)} & =\sum_{k=1}^{i-1} \frac{\rho_{\alpha, k}}{S} \boldsymbol{\Xi}_{\alpha}^{*} \mathbf{H}_{\alpha, k} \mathbf{v}_{\alpha, k}^{(l+1)} \mathbf{v}_{\alpha, k}^{(l+1) *} \mathbf{H}_{\alpha, k}^{*} \boldsymbol{\Xi}_{\alpha} \\
& +\sum_{j=i+1}^{K} \frac{\rho_{\alpha, j}}{S} \boldsymbol{\Xi}_{\alpha}^{*} \mathbf{H}_{\alpha, j} \mathbf{v}_{\alpha, j}^{(l)} \mathbf{v}_{\alpha, j}^{(l) *} \mathbf{H}_{\alpha, j}^{*} \boldsymbol{\Xi}_{\alpha} \\
& +\left(\sum_{\beta} \sum_{j=1}^{K} \frac{\rho_{\beta, j}}{S} \boldsymbol{\Xi}_{\alpha}^{*} \mathbf{G}_{\beta, j}\left(\frac{1}{N_{t}} \mathbf{I}\right) \mathbf{G}_{\beta, j}^{*} \boldsymbol{\Xi}_{\alpha}\right)+\left(1+\mathrm{INR}_{\mathrm{rem}}\right) \mathbf{I}
\end{aligned}
$$

and then we update the precoders with updated $\mathbf{E}_{\alpha, i}^{(l)}$

$$
\begin{aligned}
& \mathbf{v}_{\alpha, i}^{(l+1)}= \\
& \quad \max \operatorname{eig} \operatorname{vec}\left(\mathbf{H}_{\alpha, i}^{*} \boldsymbol{\Xi}_{\alpha}\left(\frac{\rho_{\alpha, i}}{S}\right) \mathbf{E}_{\alpha, i}^{(l)^{-2}} \boldsymbol{\Xi}_{\alpha}^{*} \mathbf{H}_{\alpha, i}, \quad \mathbf{I}+\mathbf{H}_{\alpha, i}^{*} \boldsymbol{\Xi}_{\alpha}\left(\frac{\rho_{\alpha, i}}{S}\right) \mathbf{E}_{\alpha, i}^{(l)^{-1}} \boldsymbol{\Xi}_{\alpha}^{*} \mathbf{H}_{\alpha, i}\right)
\end{aligned}
$$

subject to : $\left\|\mathbf{v}_{\alpha, i}^{(l+1)}\right\|=1$.

The iteration continues until $\epsilon=\mathrm{MSE}_{\alpha}^{(l+1)}-\mathrm{MSE}_{\alpha}^{(l)} \leq 0.001$. After finding the precoding vectors, the BS generates the postcoders,

$$
\begin{gathered}
\mathbf{u}_{\alpha, i}^{(l+1)}=\boldsymbol{\Xi}_{\alpha} \mathbf{w}_{\alpha, i}^{(l+1)^{*}} \\
\text { such that }\left\|\mathbf{u}_{\alpha, i}^{(l+1)}\right\|=1,
\end{gathered}
$$

where

$$
\mathbf{w}_{\alpha, i}^{(l+1)}=\mathbf{v}_{\alpha, i}^{(l+1) *} \mathbf{H}_{\alpha, i}^{*} \boldsymbol{\Xi}_{\alpha}\left(\sqrt{\frac{\rho_{\alpha, i}}{S}}\right) \hat{\mathbf{T}}_{\alpha, i}^{(l+1)^{-1}}
$$

and

$$
\begin{aligned}
\hat{\mathbf{T}}_{\alpha}^{(l+1)} & =\left(1+\mathrm{INR}_{\mathrm{rem}}\right) \mathbf{I}+\left(\sum_{j=1}^{K} \frac{\rho_{\alpha, j}}{S} \boldsymbol{\Xi}_{\alpha}^{*} \mathbf{H}_{\alpha, j} \mathbf{v}_{\alpha, j}^{(l+1)} \mathbf{v}_{\alpha, j}^{(l+1) *} \mathbf{H}_{\alpha, j}^{*} \boldsymbol{\Xi}_{\alpha}\right) \\
& +\left(\sum_{\beta} \sum_{j=1}^{K} \frac{\rho_{\beta, j}}{S} \boldsymbol{\Xi}_{\alpha}^{*} \mathbf{G}_{\beta, j}\left(\frac{1}{N_{t}} \mathbf{I}\right) \mathbf{G}_{\beta, j}^{*} \boldsymbol{\Xi}_{\alpha}\right) .
\end{aligned}
$$

Finally, the transmitter feeds back the optimum precoders to the user to cancel the intracell interference. 


\subsubsection{Summary of the Equations For the Uplink}

Here we summarise the required precoder and postcoder equations for each uplink optimised transceiver method.

Table 6.1: Summary of the Equations for Uplink Optimised Transceivers

\begin{tabular}{|l|l|l|}
\hline \multicolumn{3}{|c|}{ Equations } \\
\hline Optimised Transceivers & Precoder & Postcoder \\
\hline with MMSE Rx & $(6.14)$ & $(6.15)$ \\
\hline with Combined Rx & $(6.32)$ & $(6.33)$ \\
\hline
\end{tabular}

\subsubsection{Performance Parameters for Uplink Optimised Transceivers}

The SINR for user $k$ in cell $\alpha$ is expressed as

$$
\operatorname{SINR}_{\alpha, k}=\frac{\left(\frac{\rho_{\alpha, k}}{S}\right) \mathbf{u}_{\alpha, k}^{*} \mathbf{H}_{\alpha, k} \mathbf{v}_{\alpha, k} \mathbf{v}_{\alpha, k}^{*} \mathbf{H}_{\alpha, k}^{*} \mathbf{u}_{\alpha, k}}{\Delta_{\alpha, k}+\Omega_{\alpha, k}+\Lambda_{\alpha, k}},
$$

where $\Delta_{\alpha, k}$ is the dominant interference expressed as

$$
\Delta_{\alpha, k}=\mathbf{u}_{\alpha, k}^{*} \sum_{\beta}\left(\frac{\rho_{\beta, k}}{S}\right) \mathbf{G}_{\beta, k}\left[\begin{array}{lllll}
\mathbf{v}_{\beta, 1} & \mathbf{v}_{\beta, 2} & \ldots & \mathbf{v}_{\beta, K}
\end{array}\right]\left[\begin{array}{llll}
\mathbf{v}_{\beta, 1} & \mathbf{v}_{\beta, 2} & \ldots & \mathbf{v}_{\beta, K}
\end{array}\right]^{*} \mathbf{G}_{\beta, k}^{*} \mathbf{u}_{\alpha, k} .
$$

$\Omega_{\alpha, k}$ is the power of the remaining interference and noise. Note that channels of remaining interference (denoted as $\mathbf{G}_{\beta, k}^{r}$ ) are independent from those of dominant interference, as described in Chapter 4. The expectation of the remaining interference is given by

$$
\mathrm{INR}_{\mathrm{rem}}=\mathbb{E}\left\{\operatorname{tr}\left(\sum_{\beta} \frac{\rho_{\beta, k}^{r}}{S} \mathbf{G}_{\beta, k}^{r} \mathbf{G}_{\beta, k}^{r *}\right)\right\} .
$$

We assume that remaining interference is simply treated as noise with variance $\mathrm{INR}_{\text {rem. }}$. We thus calculate the average power of remaining interference and noise using

$$
\Omega_{\alpha, k}=\mathbf{u}_{\alpha, k}^{*}\left(1+\mathrm{INR}_{\mathrm{rem}}\right) \mathbf{I} \mathbf{u}_{\alpha, k} .
$$

The power of intracell interference is given by

$$
\Lambda_{\alpha, k}=\frac{\rho_{\alpha, k}}{S} \mathbf{u}_{\alpha, k}^{*} \mathbf{H}_{\alpha, k}\left(\sum_{i=1, i \neq k}^{S} \mathbf{v}_{\alpha, i} \mathbf{v}_{\alpha, i}^{*}\right) \mathbf{H}_{\alpha, k}^{*} \mathbf{u}_{\alpha, k} .
$$




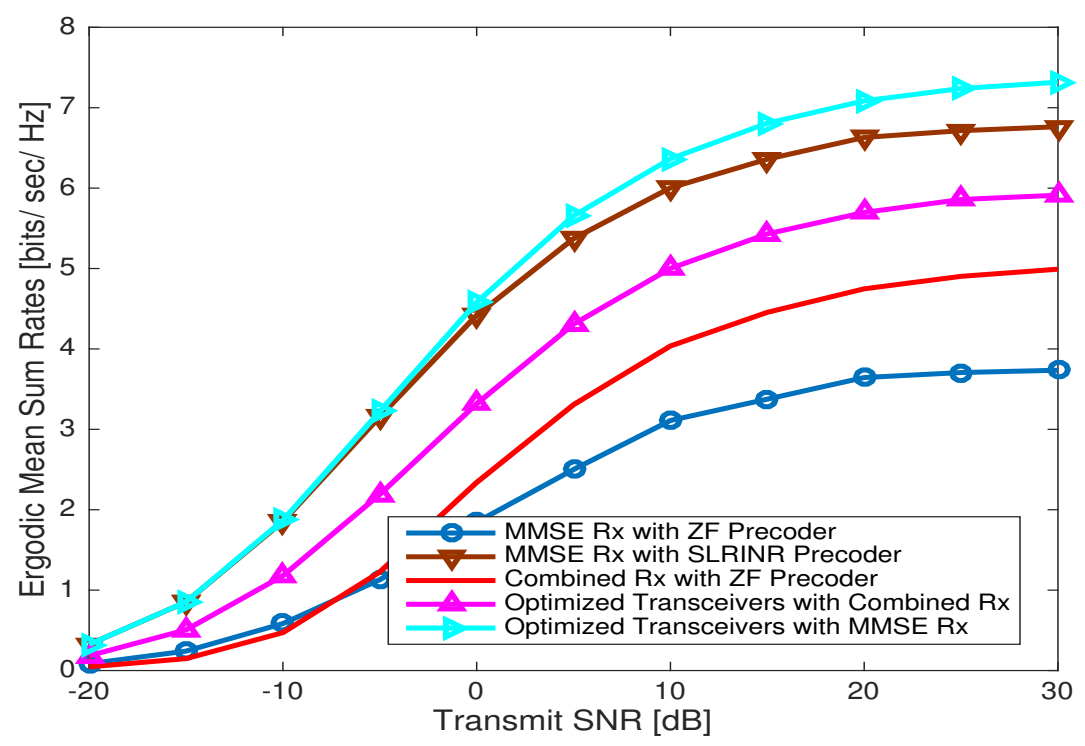

Figure 6.3: Ergodic mean sum rates for random user locations (Scenario 1 Uplink multicell networks)

The ergodic rate per bandwidth for each user is given by

$$
\mathrm{R}_{\alpha, k}=\mathbb{E}\left\{\log _{2}\left(1+\operatorname{SINR}_{\alpha, k}\right)\right\}
$$

from which we find the ergodic mean sum rates for cell $\alpha$,

$$
\tilde{\mathrm{R}}_{\alpha}=\sum_{k=1}^{S} \mathrm{R}_{\alpha, k}
$$

\subsubsection{Simulation Results}

In this section, we give the simulation results for the performance of the optimised transceivers for the scenarios defined in Chapter 2.

\section{Scenario 1: Randomly Located Users}

We first evaluate the simulations for users which are located randomly within the cell, using COST 231 Hata Urban Model [96] given for uplink systems in Chapter 2. In Fig. 6.3, we compare the optimised transceiver techniques with previous techniques mentioned earlier specifically MMSE receiver with ZF (Section 4.2.1) and SLRINR precoders (Section 5.2.3). We assume that each cell has 3 users. Here, we have assumed that each user is equipped with $N_{t}=3$ antennas. We have also assumed that CCSI from the users in 3 adjacent cells is available. Because we consider 2 adjacent cell users to be ZF'd with the combined receivers (6 users in total), each BS is equipped with $N_{r}=36$ antennas. We calculate the ergodic mean sum rates using (6.43). As seen in Fig. 


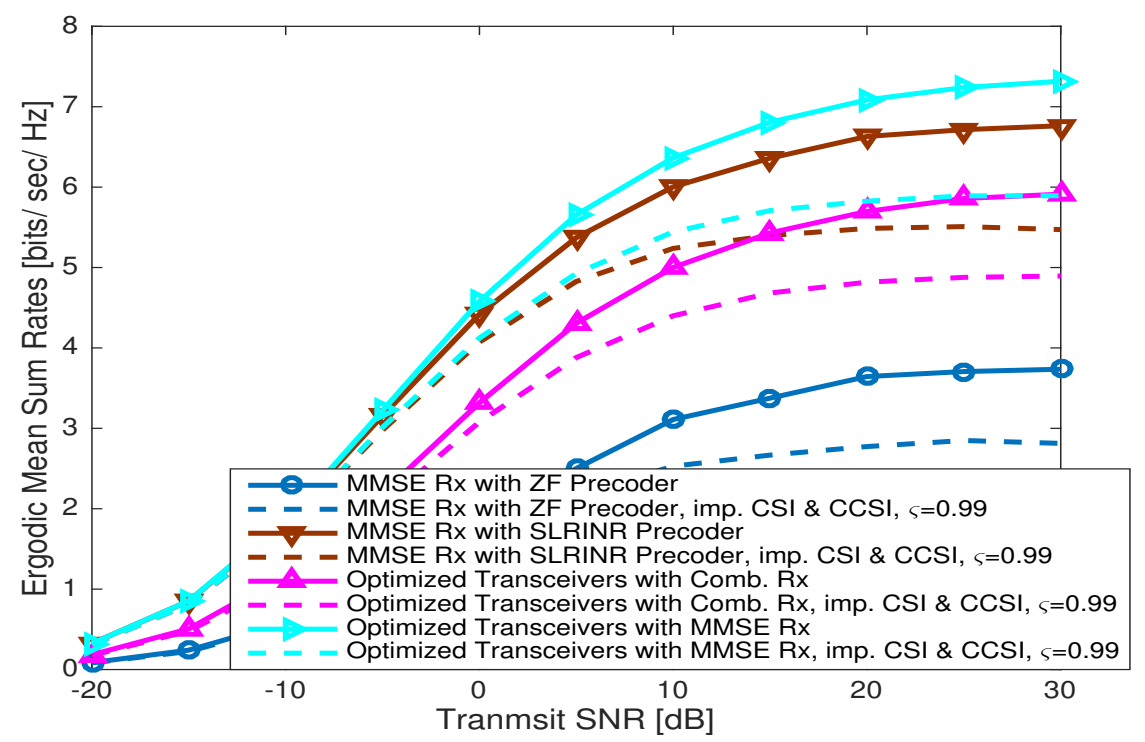

Figure 6.4: Ergodic mean sum rates for random user locations with imperfect CSI and CCSI (Scenario 1 - Uplink multicell networks)

6.3, the optimised transceivers with MMSE receiver provides the best ergodic mean sum rates, where as the MMSE receiver with ZF precoders provide the lowest mean sum rates among all techniques studied. We also observe that the optimised transceiver with combined receivers provide poorer performance than optimised transceiver with MMSE receivers. The reason is the fact that ZF part of the receiver enhances the noise and also the remaining interference as in (6.32). Similarly, we observe that the MMSE receivers with SLRINR precoders provide reasonable high mean sum rates considering that there is no iteration applied.

Next, we consider imperfect CSI and CCSI as in (2.23) and (2.24). Fig. 6.4 indicates that the optimised transceivers with MMSE receivers achieve the best ergodic mean sum rates with imperfect CSI and CCSI where $\varsigma=0.99$. The optimised transceivers could achieve almost $7.3 \mathrm{bits} / \mathrm{sec} / \mathrm{Hz}$ mean sum rate when transmit SNR is $30 \mathrm{~dB}$ and perfect CSI and CCSI is available. However, when we consider erroneous channel estimation with $\varsigma=0.99$, the ergodic mean sum rate for the optimised transceivers decreases to approximately $5.9 \mathrm{bits} / \mathrm{sec} / \mathrm{Hz}$. Considering imperfect CSI and CCSI, the optimised transceivers could achieve almost $95 \%$ higher than what MMSE receiver with ZF precoders could achieve.

\section{Scenario 2: Cell Edge Users}

Next, we consider users located in the cell-edge, using the COST 231 simulations given in Chapter 2. As cell-edge users suffer remaining interference more than other users, in Fig. 6.5 the difference between the optimised transceiver with MMSE receivers and the MMSE receivers with SLRINR precoders is not as significant as for randomly located users in Fig. 6.3. However, the optimised 


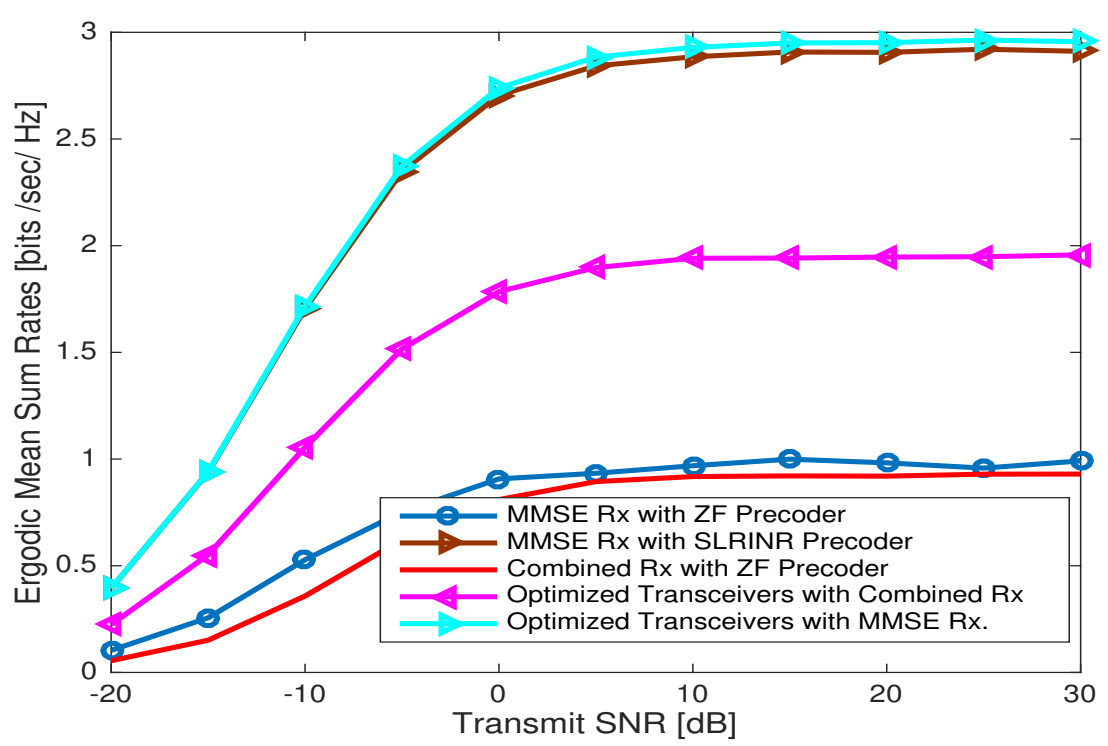

Figure 6.5: Ergodic mean sum rates for cell-edge locations (Scenario 2 - Uplink multicell networks)

transceivers with MMSE receivers still provide the highest ergodic mean sum rates among all techniques considered here. As discussed in Chapter 2, the cell edge users suffer from stronger remaining interference which is simply treated as noise. Therefore at high transmit SNR, the system can be considered similar to a noise - limited system. Because the ZF part of the combined receiver enhances the noise, it does not perform well in noise - limited environments, as discussed in Chapter 4. Therefore, the combined receivers here perform poorer than MMSE receivers. It is also observed that the optimised transceivers with combined receivers provide lower ergodic mean sum rates compared to optimised transceivers with MMSE receivers. The reason is the fact that the ZF part of the combined receiver within the iteration is enhancing the remaining interference and noise.

In Fig. 6.6, we assume erroneous channel estimation. Considering imperfect CSI and CCSI, we observe a degradation in ergodic mean sum rates, however, the optimised transceivers with MMSE receivers provide the highest ergodic mean sum rates. Fig. 6.6 also indicates that the optimised transceivers using MMSE receivers with imperfect CSI and CCSI, perform slightly better than MMSE receiver with SLRINR precoders with perfect CSI and CCSI. However, because the remaining interference is strong for cell-edge users, the optimised transceivers' gain over MMSE receiver with SLRINR precoders is very minor. 


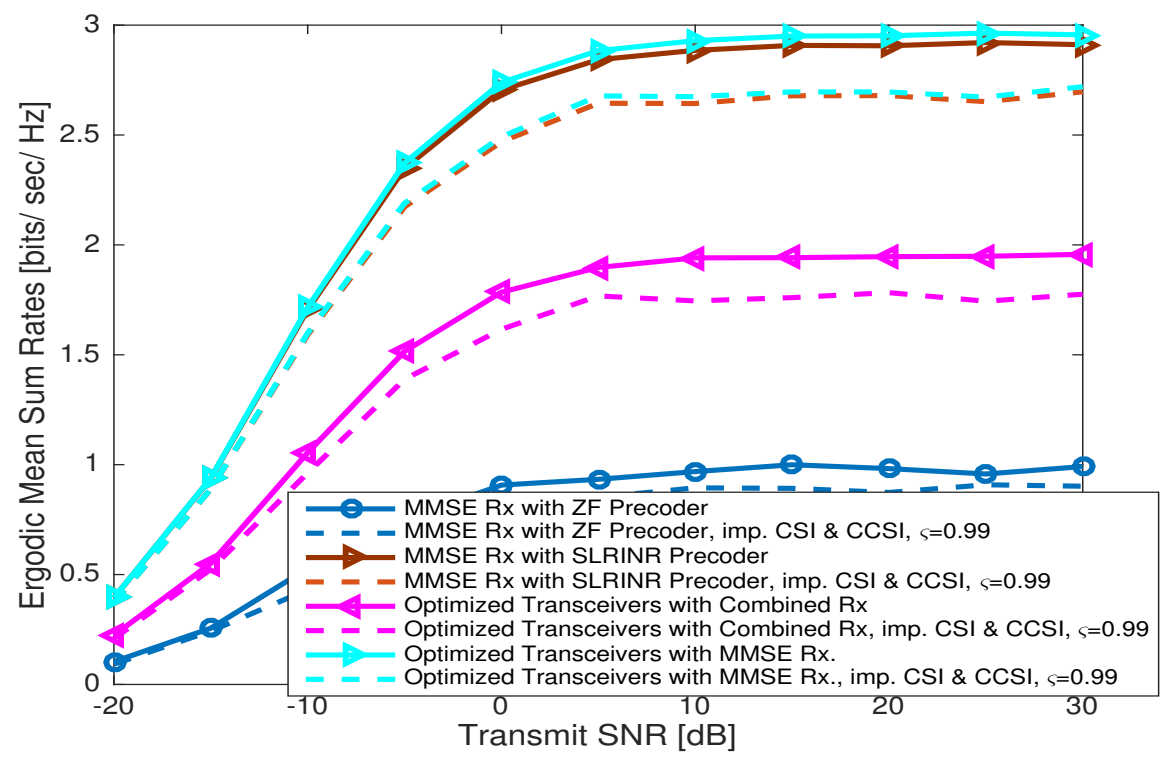

Figure 6.6: Ergodic mean sum rates for cell-edge users with imperfect CSI and CCSI (Scenario 2 - Uplink multicell networks)

\subsection{Optimised Transceivers for Multicell Down- link Networks}

We now propose novel optimised transceivers for multicell downlink systems. Similar to the uplink, we set the optimised transceivers minimising the total MSE at the BS. Because the optimisation is done at the BS, the proposed scheme does not require iterative feedback between the base station and the users.

The contributions of this section are as follows

- We propose novel optimised transceivers which minimise total MSE in the cell, considering remaining interference. We compare the performance of the proposed system with the technique of [3] and other techniques studied in Chapter 5.

- We investigate how imperfect CSI and CCSI impact the ergodic mean sum rates, considering cellular scenarios given in Chapter 2, such as cell edge and random user locations. We demonstrate via simulations that the proposed optimised transceivers still outperform the technique of [3] and the techniques in Section 5.2.3 significantly under the assumption of channel estimation errors. 


\subsubsection{The System Model}

We assume a multicellular downlink network. Each BS transmits its data using $K$ precoding vectors, $\mathbf{B}_{\alpha}=\left[\mathbf{v}_{\alpha, 1}, \mathbf{v}_{\alpha, 2}, \ldots, \mathbf{v}_{\alpha, K}\right] \in \mathbb{C}^{N_{t} \times K}$. The BS $\alpha$ sends the complex symbols $\left(x_{\alpha, 1}, x_{\alpha, 2}, \ldots, x_{\alpha, K}\right)$, intended for the users in cell $\alpha$. The received signal of user $k$ in cell $\alpha$ is given as

$\mathbf{y}_{\alpha, k}=\left(\sqrt{\frac{\rho_{\alpha, k}}{S}}\right) \mathbf{H}_{\alpha, k} \sum_{i=1}^{K} \mathbf{v}_{\alpha, i} x_{\alpha, i}+\sum_{\beta}\left(\sqrt{\frac{\rho_{\beta, k}}{S}}\right) \mathbf{G}_{\beta, k} \sum_{l=1}^{K} \mathbf{v}_{\beta, l} x_{\beta, l}+\mathbf{z}_{\alpha, k}+\mathbf{n}_{\alpha, k}$,

where $\mathbf{z}_{\alpha, k}$ is the remaining interference and $\mathbf{n}_{\alpha, k}$ is the AWGN noise. Here we note that the main difference between (6.44) and (5.23) in Chapter 5 is the fact that 6.44 does not use secondary precoding matrix $\overline{\mathbf{P}}$ as it optimises the whole system. The received signal is multiplied with the postcoding vector, $\mathbf{u}_{\alpha, k}$, giving the desired signal

$$
\tilde{x}_{\alpha, k}=\mathbf{u}_{\alpha, k}^{*} \mathbf{y}_{\alpha, k} .
$$

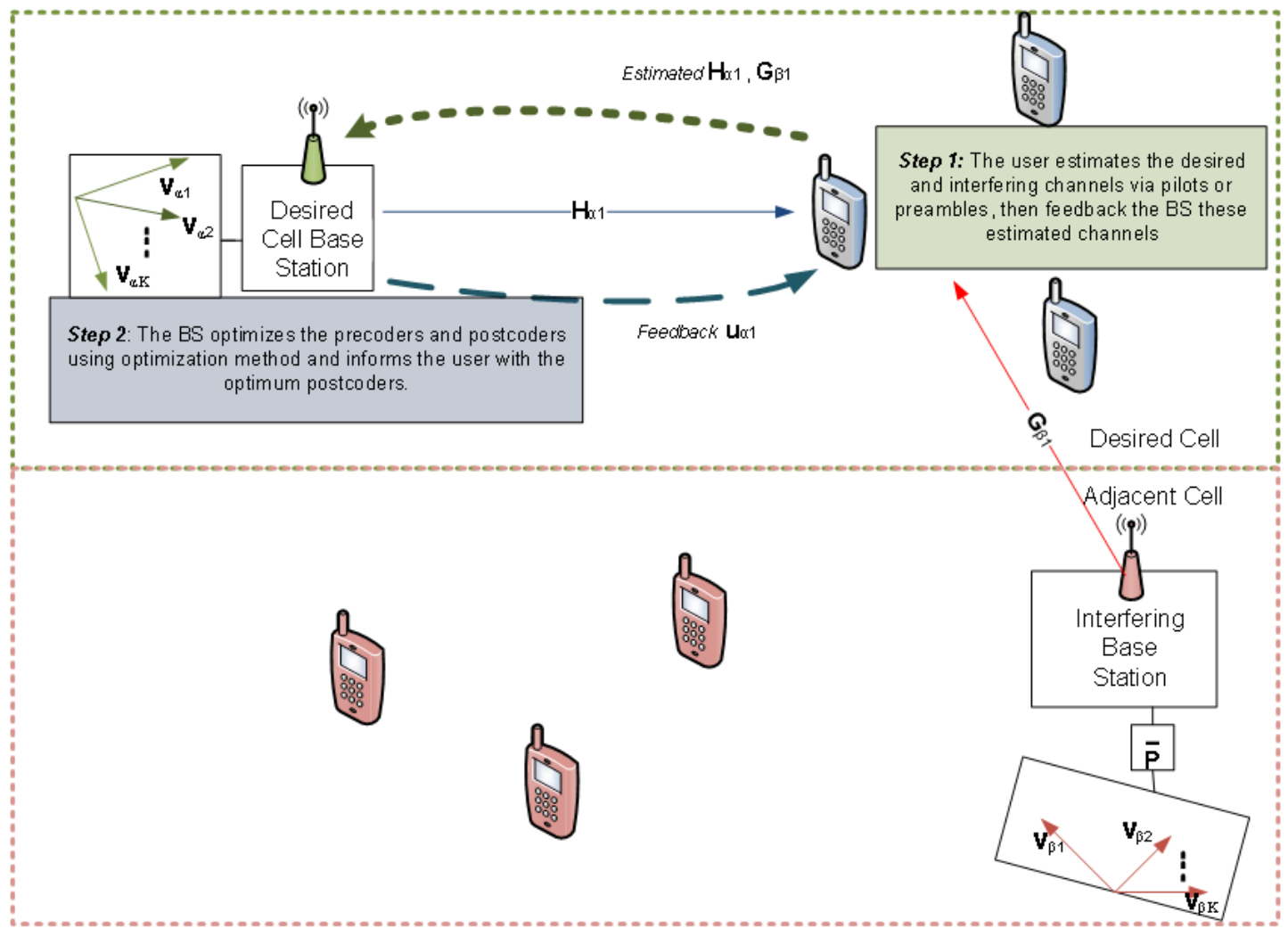

Figure 6.7: System model of the proposed optimised transceivers for multicell downlink networks 


\subsubsection{The Algorithm of the Optimised Transceivers for Downlink Networks}

In this section, we give the algorithm of our proposed optimised transceivers. We find the precoder and postcoder set for each user that minimises the total MSE at the BS. After the optimisation process at the BS, the BS informs each user of their optimum postcoders. The system model is given in Fig. 6.7.

\section{The Algorithm}

Each step of the proposed algorithm is explained here in detail.

- Initialise the precoders and postcoders: We first initialise the precoders,

$$
\mathbf{v}_{\alpha, k}^{(0)}=\max \text { eig. vector of }\left(\mathbf{H}_{\alpha, k}^{*} \tilde{\boldsymbol{\Phi}}_{\alpha, k} \mathbf{H}_{\alpha, k}\right),
$$

where $\tilde{\boldsymbol{\Phi}}_{\alpha, k}=\left(1+\mathrm{INR}_{\mathrm{rem}}\right) \mathbf{I}+\sum_{\beta} \frac{\rho_{\beta, k}}{S}\left(\mathbf{G}_{\beta, k} \mathbb{E}\left\{\mathbf{B}_{\beta} \mathbf{B}_{\beta}^{*}\right\} \mathbf{G}_{\beta, k}^{*}\right)$ and $\mathbb{E}\left\{\mathbf{B}_{\beta} \mathbf{B}_{\beta}^{*}\right\}=$ $\frac{S}{N_{t}} \mathbf{I}$, as found via simulations. Then, we write the initial value of the postcoder vector as follows

$$
\mathbf{u}_{\alpha, k}^{(0)}=\mathbf{v}_{\alpha, k}^{(0)^{*}} \mathbf{H}_{\alpha, k}^{*}\left(\sqrt{\frac{\rho_{\alpha, k}}{S}}\right) \mathbf{T}_{\alpha, k}^{(0)^{-1}}
$$

where

$$
\begin{aligned}
\mathbf{T}_{\alpha, k}^{(0)} & =\left(1+\mathrm{INR}_{\mathrm{rem}}\right) \mathbf{I}+\frac{\rho_{\alpha, k}}{S} \sum_{j=1}^{K} \mathbf{H}_{\alpha, k} \mathbf{v}_{\alpha, j}^{(0)} \mathbf{v}_{\alpha, j}^{(0) *} \mathbf{H}_{\alpha, i}^{*} \\
& +\sum_{\beta} \frac{\rho_{\beta, k}}{S} \mathbf{G}_{\beta, k}\left(\frac{S}{N_{t}} \mathbf{I}\right) \mathbf{G}_{\beta, k}^{*} .
\end{aligned}
$$

- Optimisation: We find the optimum precoders and postcoders that minimise the total MSE. We compute MSE for each user following a similar way of [85]

$$
\operatorname{MSE}_{\alpha, k}^{(0)}=1-\mathbf{v}_{\alpha, k}^{(0) *} \mathbf{H}_{\alpha, k}^{*}\left(\frac{\rho_{\alpha, k}}{S}\right) \mathbf{T}_{\alpha, k}^{(0)^{-1}} \mathbf{H}_{\alpha, k} \mathbf{v}_{\alpha, k}^{(0)} .
$$

Total $\mathrm{MSE}_{\alpha}$ for cell $\alpha$ can be computed as follows

$$
\operatorname{MSE}_{\alpha}^{(0)}=\sum_{k=1}^{K} \operatorname{MSE}_{\alpha, k}^{(0)}
$$

Next, we note that using the matrix inversion lemma, $\mathbf{T}_{\alpha, k}^{(0)^{-1}}$ can be ex- 
pressed as

$$
\mathbf{T}_{\alpha, k}^{(0)^{-1}}=\mathbf{E}_{\alpha, k}^{(0)^{-1}}-\frac{\mathbf{E}_{\alpha, k}^{(0)^{-1}} \mathbf{H}_{\alpha, k} \mathbf{v}_{\alpha, k}^{(0)}\left(\frac{\rho_{\alpha, k}}{S}\right) \mathbf{v}_{\alpha, k}^{(0)^{*}} \mathbf{H}_{\alpha, k}^{*} \mathbf{E}_{\alpha, k}^{(0)^{-1}}}{1+\mathbf{v}_{\alpha, k}^{(0)^{*}} \mathbf{H}_{\alpha, k}^{*}\left(\frac{\rho_{\alpha, k}}{S}\right) \mathbf{E}_{\alpha, k}^{(0)^{-1}} \mathbf{H}_{\alpha, k} \mathbf{v}_{\alpha, k}^{(0)}}
$$

where

$$
\mathbf{E}_{\alpha, k}^{(0)}=\frac{\rho_{\alpha, k}}{S} \sum_{i=1, i \neq k}^{K} \mathbf{H}_{\alpha, k} \mathbf{v}_{\alpha, i}^{(0)} \mathbf{v}_{\alpha, i}^{(0) *} \mathbf{H}_{\alpha, k}^{*}+\left(\sum_{\beta} \frac{\rho_{\beta, k}}{N_{t}} \mathbf{G}_{\beta, k} \mathbf{G}_{\beta, k}^{*}\right)+\left(1+\mathrm{INR}_{\mathrm{rem}}\right) \mathbf{I} .
$$

Then, we can rewrite the total MSE in (6.50)

$$
\mathrm{MSE}_{\alpha}^{(0)}=K-A_{\alpha},
$$

where

$$
A_{\alpha}=\sum_{k=1}^{K} \frac{\rho_{\alpha, k}}{S} \mathbf{v}_{\alpha, k}^{(0)^{*}} \mathbf{H}_{\alpha, k}^{*}\left(\mathbf{E}_{\alpha, k}^{(0)^{-1}}-\frac{\mathbf{E}_{\alpha, k}^{(0)^{-1}} \mathbf{H}_{\alpha, k} \mathbf{v}_{\alpha, k}^{(0)}\left(\frac{\rho_{\alpha, k}}{S}\right) \mathbf{v}_{\alpha, k}^{(0)^{*}} \mathbf{H}_{\alpha, k}^{*} \mathbf{E}_{\alpha, k}^{(0)^{-1}}}{1+\mathbf{v}_{\alpha, k}^{(0)^{*}} \mathbf{H}_{\alpha, k}^{*}\left(\frac{\rho_{\alpha, k}}{S}\right) \mathbf{E}_{\alpha, k}^{(0)^{-1}} \mathbf{H}_{\alpha, k} \mathbf{v}_{\alpha, k}^{(0)}}\right) \mathbf{H}_{\alpha, k} \mathbf{v}_{\alpha, k}^{(0)}
$$

In order to minimise (6.53), we need to maximise the second term of (6.53) that we substract from $K$. Let us rewrite (6.53)

$$
\operatorname{MSE}_{\alpha}^{(0)}=K-\left(\sum_{k=1}^{K} Q_{\alpha, k}^{(0)}-\frac{Q_{\alpha, k}^{(0)^{2}}}{1+Q_{\alpha, k}^{(0)}}\right)
$$

where $Q_{\alpha, k}^{(0)}=\mathbf{v}_{\alpha, k}^{(0)^{*}} \mathbf{H}_{\alpha, k}^{*}\left(\frac{\rho_{\alpha, k}}{S}\right) \mathbf{E}_{\alpha, k}^{(0)^{-1}} \mathbf{H}_{\alpha, k} \mathbf{v}_{\alpha, k}^{(0)}$. We note that $Q_{\alpha, k}^{(0)}-\frac{Q_{\alpha, k}^{(0)^{2}}}{1+Q_{\alpha, k}^{(0)}}=$ $1-\frac{1}{1+Q_{\alpha, k}^{(0)}}$. Therefore we can rewrite $(6.53)$

$$
\begin{aligned}
\operatorname{MSE}_{\alpha}^{(0)} & =K-\sum_{k=1}^{K}\left(1-\frac{1}{1+Q_{\alpha, k}^{(0)}}\right) \\
& =K-K+\sum_{k=1}^{K}\left(\frac{1}{1+Q_{\alpha, k}^{(0)}}\right) \\
& =\sum_{k=1}^{K} \frac{1}{1+\mathbf{v}_{\alpha, k}^{(0)^{*}} \mathbf{H}_{\alpha, k}^{*}\left(\frac{\rho_{\alpha, k}}{S}\right) \mathbf{E}_{\alpha, k}^{(0)^{-1}} \mathbf{H}_{\alpha, k} \mathbf{v}_{\alpha, k}^{(0)}} .
\end{aligned}
$$

We can minimise (6.56) maximising its denominator. Using the Rayleigh Quotinent Theorem [85,120], we find the precoding vectors that minimise 
(6.56) as follows

$$
\begin{aligned}
\mathbf{v}_{\alpha, k}^{(1)} & =\max \text { eig vector of }\left(\mathbf{H}_{\alpha, k}^{*}\left(\frac{\rho_{\alpha, k}}{S}\right) \mathbf{E}_{\alpha, k}^{(0)^{-1}} \mathbf{H}_{\alpha, k}\right) \\
\text { such that } & : \quad\left\|\mathbf{v}_{\alpha, k}^{(1)}\right\|=1 .
\end{aligned}
$$

- Iteration: We can find $\mathbf{E}_{\alpha, k}$ at $l$ th iteration using the updated precoding vectors. The iteration continues until $\epsilon=\mathrm{MSE}_{\alpha}^{(l+1)}-\mathrm{MSE}_{\alpha}^{(l)} \leq 0.001$. After convergence, the BS finds the optimum precoding vector

$$
\begin{aligned}
\mathbf{v}_{\alpha, k}^{(l+1)} & =\max \text { eig vector of }\left(\mathbf{H}_{\alpha, k}^{*}\left(\frac{\rho_{\alpha, k}}{S}\right) \mathbf{E}_{\alpha, k}^{(l)^{-1}} \mathbf{H}_{\alpha, k}\right) \\
\text { such that } & :\left\|\mathbf{v}_{\alpha, k}^{(l+1}\right\|=1 .
\end{aligned}
$$

After finding the optimum precoders, the postcoders are generated as

$$
\begin{aligned}
\mathbf{u}_{\alpha, k} & =\mathbf{v}_{\alpha, k}^{(l+1) *} \mathbf{H}_{\alpha, k}^{*}\left(\sqrt{\frac{\rho_{\alpha, k}}{S}}\right) \mathbf{T}_{\alpha, k}^{(l+1)^{-1}} \\
\text { such that } & : \quad\left\|\mathbf{u}_{\alpha, k}\right\|=1,
\end{aligned}
$$

where

$$
\begin{aligned}
\mathbf{T}_{\alpha, k}^{(l+1)} & =\left(1+\mathrm{INR}_{\mathrm{rem}}\right) \mathbf{I}+\left(\frac{\rho_{\alpha, k}}{S} \sum_{j=1}^{K_{\alpha}} \mathbf{H}_{\alpha, k} \mathbf{v}_{\alpha, j}^{(l+1)} \mathbf{v}_{\alpha, j}^{(l+1) *} \mathbf{H}_{\alpha, k}^{*}\right) \\
& +\left(\sum_{\beta} \frac{\rho_{\beta, k}}{N_{t}} \mathbf{G}_{\beta, k} \mathbf{G}_{\beta, k}^{*}\right) .
\end{aligned}
$$

Finally, the transmitter informs each user the optimum postcoders in order to cancel the interference at the receivers. Note that the iteration is performed only at the transmitter. There is only one feedback link from the user to the BS where they feed back the channel estimations to the BS.

\subsubsection{Summary of the Equations For the Downlink}

Here we summarise the required precoder and postcoder equations for each uplink optimised transceiver method.

Table 6.2: Summary of the Equations for Downlink Optimised Transceivers

\begin{tabular}{|l|l|l|}
\hline \multicolumn{3}{|c|}{ Equations } \\
\hline Optimised Transceivers & Precoder & Postcoder \\
\hline with MMSE Rx & $(6.58)$ & $(6.59)$ \\
\hline
\end{tabular}




\section{Performance Metrics for Downlink Optimised Transceivers}

The SINR for user $k$ in cell $\alpha$ is expressed as

$$
\operatorname{SINR}_{\alpha, k}=\frac{\left(\frac{\rho_{\alpha, k}}{S}\right) \mathbf{u}_{\alpha, k}^{*} \mathbf{H}_{\alpha, k} \mathbf{v}_{\alpha, k} \mathbf{v}_{\alpha, k}^{*} \mathbf{H}_{\alpha, k}^{*} \mathbf{u}_{\alpha, k}}{\Delta_{\alpha, k}+\Gamma_{\alpha, k}+\Lambda_{\alpha, k}},
$$

where $\Delta_{\alpha, k}$ is the dominant intereference given as

$$
\Delta_{\alpha, k}=\mathbf{u}_{\alpha, k}^{*} \sum_{\beta}\left(\frac{\rho_{\beta, k}}{S}\right) \mathbf{G}_{\beta, k}\left[\mathbf{v}_{\beta, 1} \mathbf{v}_{\beta, 2} \ldots \mathbf{v}_{\beta, K}\right]\left[\begin{array}{llll}
\mathbf{v}_{\beta, 1} & \mathbf{v}_{\beta, 2} & \ldots & \mathbf{v}_{\beta, K}
\end{array}\right]^{*} \mathbf{G}_{\beta, k}^{*} \mathbf{u}_{\alpha, k} .
$$

$\Gamma_{\alpha, k}$ is the power of remaining interference and noise. Note that, channels of interferers generating remaining interference (denoted as $\mathbf{G}_{\beta, k}^{r}$ ) are independent from those of dominant interferers. As mentioned in Chapter 4, we assume that remaining interference is simply treated as noise with variance $\mathrm{INR}_{\text {rem. }}$. Then we calculate the average power of remaining interference and noise as

$$
\Gamma_{\alpha, k}=\mathbf{u}_{\alpha, k}^{*}\left(1+\mathrm{INR}_{\mathrm{rem}}\right) \quad \mathbf{u}_{\alpha, k} .
$$

The power of intracell interference is obtained using

$$
\Lambda_{\alpha, k}=\frac{\rho_{\alpha, k}}{S} \mathbf{u}_{\alpha, k}^{*} \mathbf{H}_{\alpha, k}\left(\sum_{i=1, i \neq k}^{S} \mathbf{v}_{\alpha, i} \mathbf{v}_{\alpha, i}^{*}\right) \mathbf{H}_{\alpha, k}^{*} \mathbf{u}_{\alpha, k}
$$

Substituting (6.62), (6.63) and (6.64) in (6.61), the corresponding rate per unit bandwidth for user $k$ is

$$
\mathrm{R}_{\alpha, k}=\mathbb{E}\left\{\log _{2}\left(1+\mathrm{SINR}_{\alpha, k}\right)\right\}
$$

and the sum rate per unit bandwidth for cell $\alpha$ as

$$
\tilde{\mathrm{R}}_{\alpha}=\sum_{k=1}^{S} \mathrm{R}_{\alpha, k}
$$

\subsubsection{Simulation Results}

In this section, we give simulation results to compare our proposed optimised scheme with the technique (MMSE-like postcoders and ZF precoders) given in [3], as well as our other proposed techniques (SLNR and SLRINR precoders with MMSE-like postcoders) given in Section 5.2.3. We first evaluate simulations for the benchmark results considering the parameters of [3]. Then we apply our system in $3 \mathrm{GPP}$ scenarios that we defined in Chapter 2. We also give simulation 


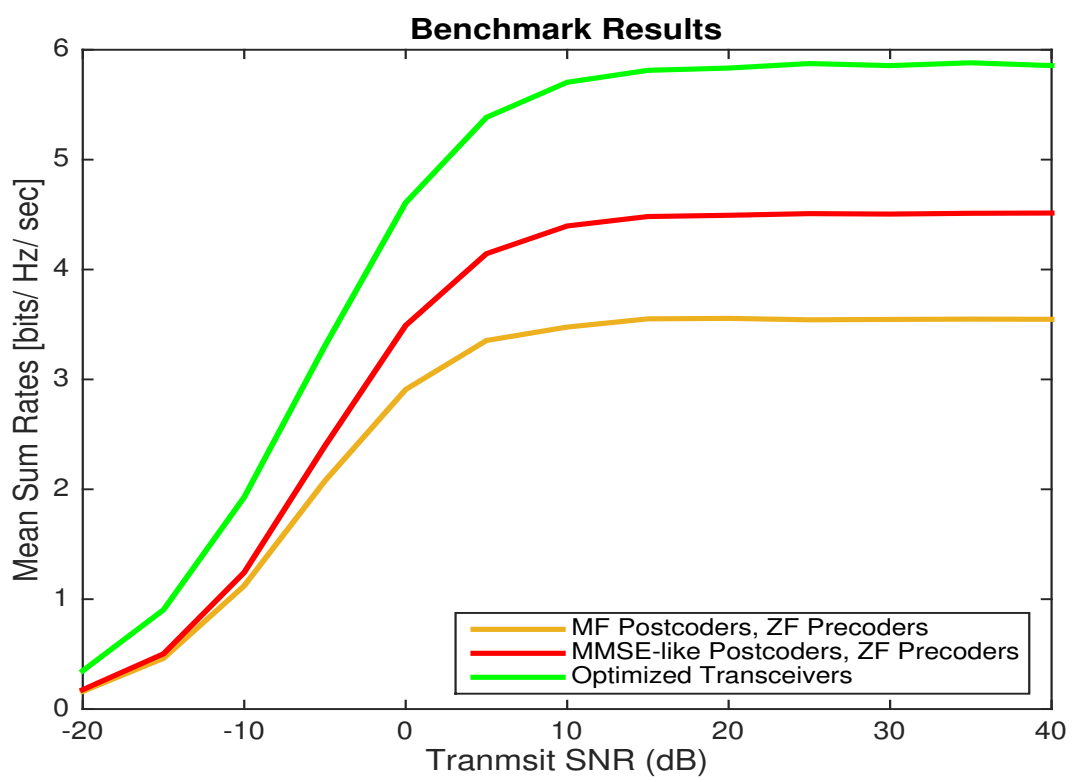

Figure 6.8: Comparison of proposed optimised transceivers with benchmark results of $[12]$

results considering imperfect CSI and CCSI.

\section{Benchmark Results}

Fig. 6.8 presents simulation results to compare our proposed optimised transceivers with the set of MMSE-like postcoders and ZF precoders that was proposed in [3]. Similar to [3], we assume that $N_{t}=N_{r}=4$ and there are $K=3$ users in each cell. Each user sends a single stream therefore total number of streams is $S=3$. We also consider that the ratio of remaining interference over dominant interference is $\gamma=0.4$ on average and only one dominant interference is considered. We assume there is only one feedback link from the user to the base station. As seen in Fig. 6.8, the proposed optimised transceivers achieve significant gain over the MMSE-like postcoders and ZF precoders of [3]. The authors of [3] claimed that, their technique achieves approximately $28 \%$ more sum rates than the MF receivers. However, our optimised transceivers outperforms both techniques. It achieves approximately $32 \%$ better sum rates than the technique of [3], and approximately $69 \%$ better than MF receiver. The reason of these significant gains is the fact that MF receiver does not use the CCSI and the remaining interference, while the technique of [3] does not use the remaining interference in generating the precoders. However, the optimised transceivers consider the remaining interference in finding the optimum precoders. 


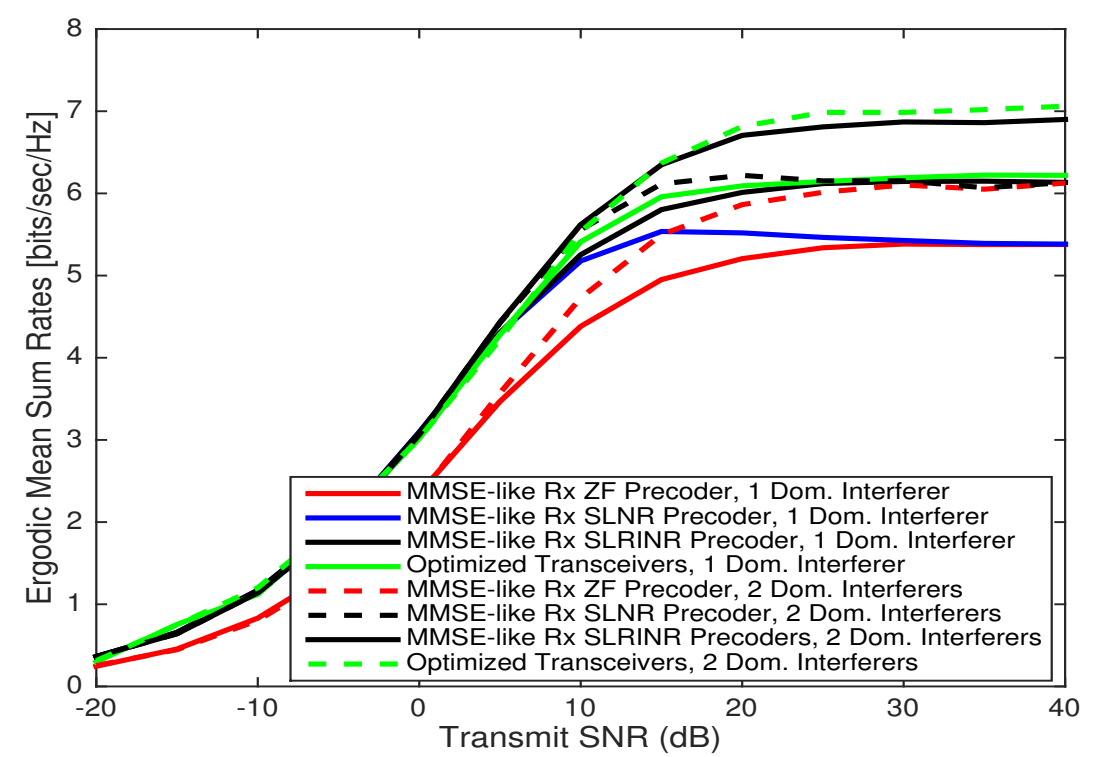

Figure 6.9: Ergodic mean sum rates for random user locations (Scenario 1 Downlink multicell networks)

\section{Using Cellular Scenarios}

Here, we assume that there are $K=3$ users in each cell. We also assume that both transmitters and receivers are equipped with $N_{t}=N_{r}=4$ antennas respectively. We consider the 19-cell hexagonal cellular network, using COST 231 Hata urban path loss model with log-normal shadowing as discussed in Chapter 2.

\section{Scenario 1: Random User Locations}

We first evaluate the simulations for random user locations. In Fig. 6.9, we give simulation results considering 1 dominant interferer case and 2 dominant interferers case. From the 19-cell simulations given in Chapter 2 , we find $\gamma \approx 1.65$ on average if we assume 1 dominant interferer, and $\gamma \approx 0.68$ on average if we assume 2 dominant interferers. Simulation results in Fig. 6.9 demonstrate that our proposed optimised transceivers outperforms the technique of [3] which is MMSE-like receivers with ZF precoders significantly. Considering 1 dominant interferer, the proposed technique achieves approximately $13 \%$ better sum rates compared to MMSE-like receivers with ZF precoders given in [3]. On the other hand, considering 2 dominant interferers, the proposed technique achieves approximately $16 \%$ over [3]. We also observe that our previously proposed technique, MMSE-like receivers with SLRINR precoders (Section 5.2.3), achieve slightly lower ergodic mean sum rates than the optimised transceivers. However, the difference between these two techniques is more prominent when we consider 2 dominant interferers. The reason is the fact that the remaining interference is lower when we consider 2 dominant interferers. As the much more 


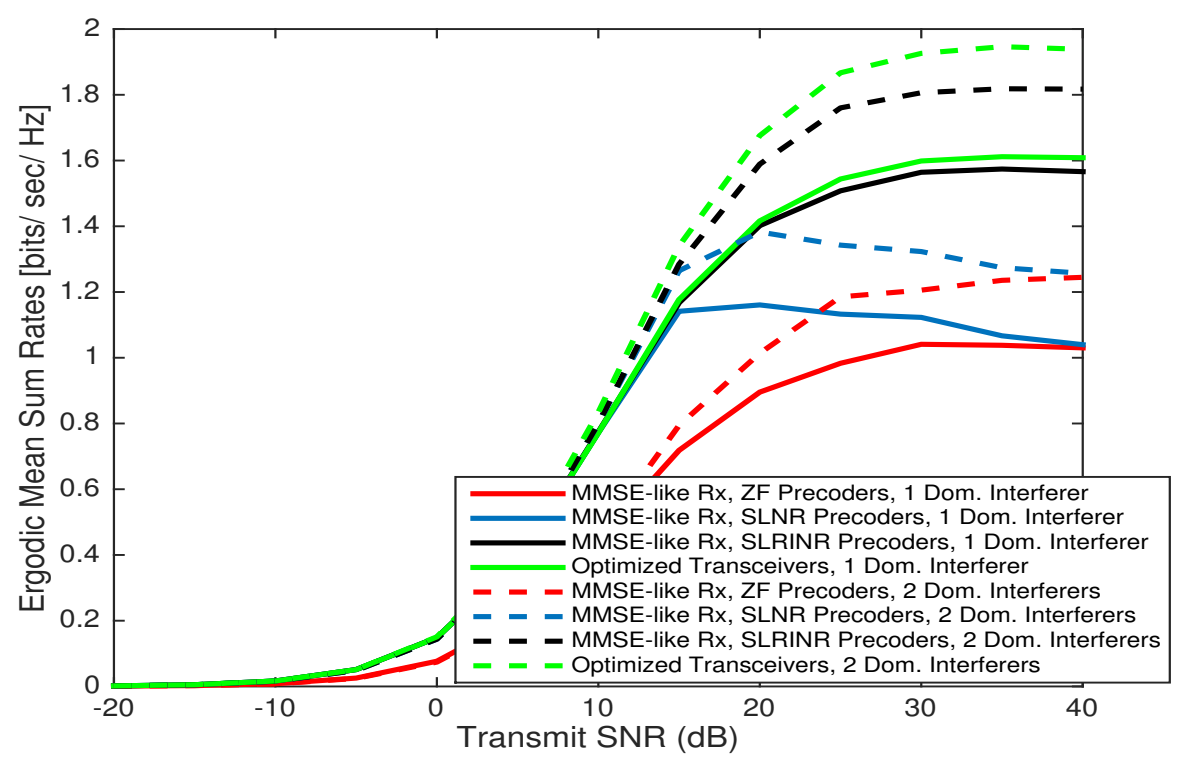

Figure 6.10: Ergodic mean sum rates for cell-edge locations (Scenario 2 - Downlink multicell networks)

of the interfering channel is known, the optimised transceivers perform better than SLRINR precoders.

\section{Scenario 2: Cell Edge Users}

Next, we specifically investigate the performance of cell-edge users. We define the cell-edge users as given in Chapter 2, Section 2.3.2. In Fig. 6.10, we demonstrate the gain that can be achieved by considering 2 dominant interferers. Simulation results indicate that the proposed optimised transceivers achieve more significant gains for cell-edge users. Considering 1 dominant interferer and transmit SNR is $40 \mathrm{~dB}$, our proposed technique achieves approximately $61 \%$ over MMSE-like receivers with ZF precoders of [3]. Considering 2 dominant interference, optimised transceivers achieve approximately $67 \%$ better sum rates than [3]. We also note that we can almost double the performance with optimised transceivers, compared to the technique of [3] considering 1 dominant interference. This is a very significant gain. We also observe that the optimised transceivers provider higher ergodic mean sum rates over MMSE receivers with SLRINR precoders. Considering 2 dominant interferers, the optimised transceivers achieve approximately $11 \%$ more ergodic mean sum rates over MMSE receivers with SLRINR precoders when transmit SNR is $40 \mathrm{~dB}$.

\section{Imperfect CSI and CCSI}

Next, we demonstrate how the proposed optimised transceivers perform with channel imperfections. In Fig. 6.11, we give results for randomly located users. We consider 2 dominant interferers. We assume all channel estimations are 


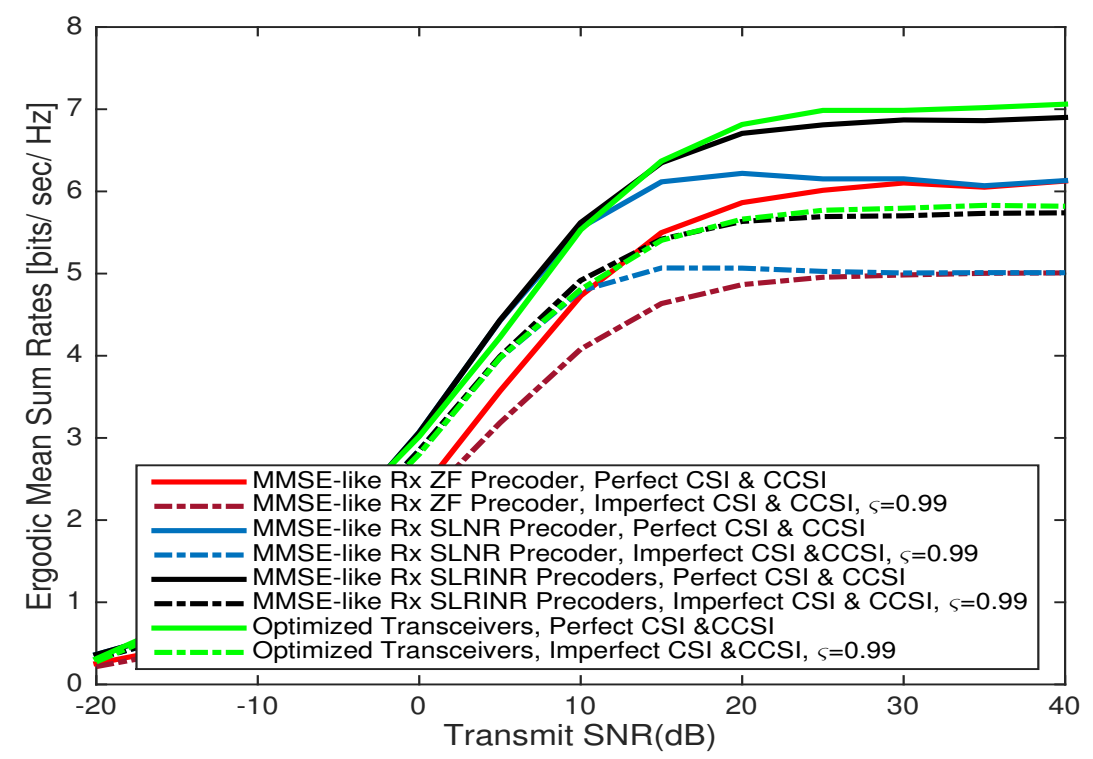

Figure 6.11: Ergodic mean sum rates with imperfect CSI and CCSI for random user locations (Scenario 1 - Downlink multicell networks)

erroneous (direct channels and cross channels) and the imperfection parameter $\varsigma$ in (2.23) and (2.24) is assumed to be $\varsigma=0.99$. The results indicate that all of the techniques suffer from imperfect channel estimation. However, the optimised transceivers are shown to achieve the highest ergodic mean sum rates among the techniques compared.

Finally, the simulations are evaluated for cell-edge users who are expected to suffer from channel imperfections more than any other user in the cell. We again consider 2 dominant interferers case. We also assume the imperfect CSI and CCSI case where $\varsigma=0.99$. As seen in Fig. 6.12, all techniques suffer from channel imperfections drastically. However, it is also shown that the optimised receivers achieve the highest ergodic mean sum rates among all techniques. When transmit SNR is $40 \mathrm{~dB}$, the optimised transceivers achieve almost $50 \%$ higher ergodic mean sum rates over both ZF precoders and SLNR precoders, approximately $12 \%$ over SLRINR precoders.

\subsection{Summary}

In this chapter we have investigated the optimization techniques for both uplink and downlink multicellular networks. First, we have proposed novel optimised transceivers for multicell uplink networks. We have derived optimum precoderpostcoder set that minimizes the total MSE at the base station. We have shown that optimised transceiver achieves significantly better results compared to our scheme, Combined Receivers with ZF Precoders of Section 4.2.1. We have also 


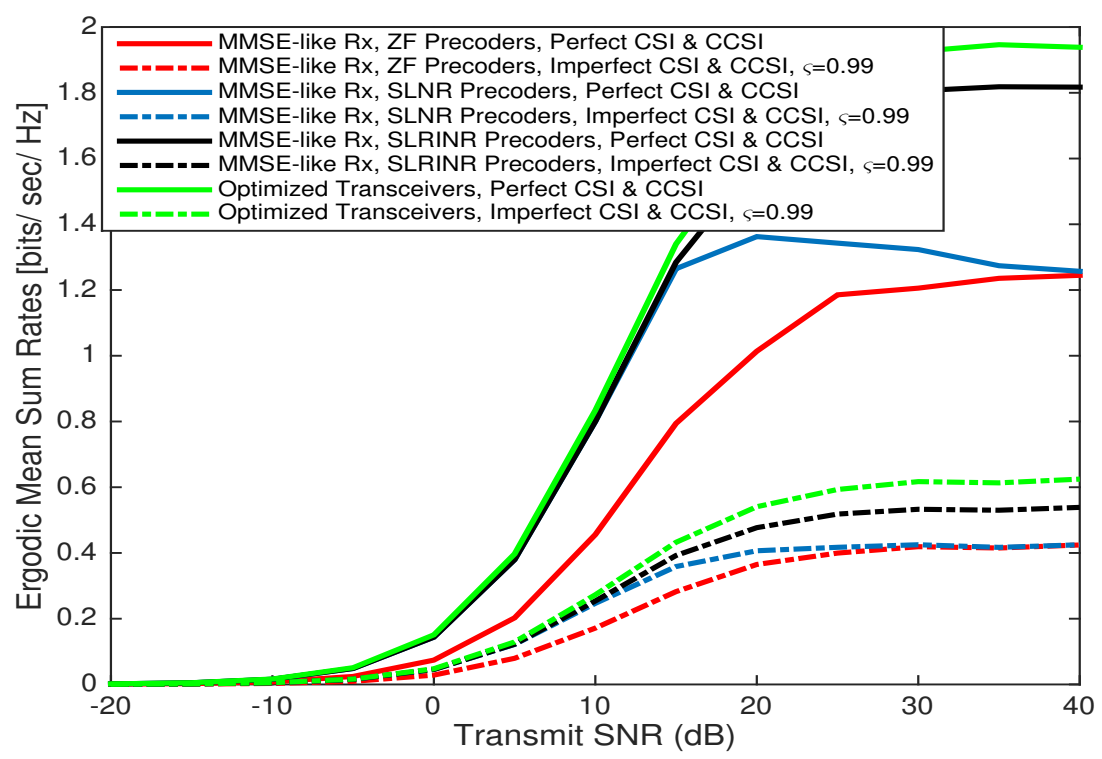

Figure 6.12: Ergodic mean sum rates with imperfect CSI and CCSI for cell-edge locations (Scenario 2 - Downlink multicell networks)

considered implementing the combined receivers in the optimisation. We have shown that optimised transceivers with combined receivers perform well, however, not better than the optimised transceivers with MMSE receivers, because the $\mathrm{ZF}$ part of the combined receiver is enhancing the remaining interference and noise. However it still provides higher ergodic mean-sum rates compared to non-optimised transceivers, the MMSE-receiver with ZF precoders and the combined receiver with ZF precoders. We have also considered MMSE receivers with SLRINR precoders that we have proposed in Section 5.2.3. The simulation results have indicated that the optimised transceivers have also outperform MMSE receivers with SLRINR precoders, at high transmit SNR. Furthermore, we have considered cell-edge users only, using the 3GPP scenarios given in Chapter 2. We have also considered imperfect channel state information. As cell-edge users suffer from interference drastically, compared to other users, the gain that can be achieved with optimised transceivers is more prominent. Optimised transceivers with MMSE receivers have been shown to almost double the ergodic mean sum rates over MMSE receivers with ZF precoders. Later, we have considered imperfect CSI and CCSI. We have also demonstrated that for any case, the proposed optimised transceivers with MMSE receivers outperform the other techniques significantly.

Following the uplink networks, we have proposed novel optimised transceivers for multicellular downlink networks as well. We have derived optimum precoderpostcoder set that minimizes the total MSE at the base station. We have shown that optimised transceiver outperforms the existing scheme of [3]. Especially 
for cell-edge users, we have demonstrated that it is possible to get gains more than $60 \%$. Furthermore, we have shown that we can even double the mean sum rates of [3] with optimised transceivers considering 2 dominant interferers for cell-edge users. We have also considered imperfect CSI and CCSI for randomly located users and cell-edge users. We have observed that the proposed optimised transceivers are more prone to suffer imperfect channel estimations because the error accumulates with the iterations. However, we have also demonstrated that for any case, the proposed optimised transceiver outperforms the technique of [3] significantly. 


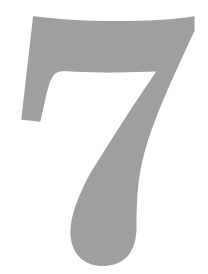

\section{User Selection and Power Adapta- tion Techniques}

Spectral efficiency of a multi-user MIMO system can be improved with user selection diversity and power adaptation techniques. In this chapter, we study some user selection and power adaptation schemes in order to apply to the IAC techniques. The main contributions of this chapter are

- We compare several user selection schemes that we have applied to our proposed optimized transceivers. We show that the ergodic mean sum rates can be improved significantly with selecting users, even with imperfect CSI and CCSI.

- We also study power adaptation schemes. We show that adapting the power based on estimated rates and SLRINR provide better ergodic mean sum rates relative to the corresponding values from uniform power adaptation.

\subsection{Motivation and Related Work}

User selection and power adaptation are commonly used in many kinds of systems where demand is high or low. They are essential when the potential user demand exceeds the available transmission resources. Prudent user selection will help ensure high system throughput by avoiding users with high interference and low received signal power [121]. However, selecting users opportunistically to maximize sum-rate will result in unequal rates provided to a single user. This leads us to apply user scheduling under a fairness constraint [122]. However, 
fairness is not the only issue. The major issues in user selection can be addressed as follows [123]

- Wireless link variability: Contrary to wireline networks, wireless transmissions have high variability [123]. Due to characteristics of wireless channels which are discussed in Chapter 2, the wireless channels are more prone to errors and suffer from interference, fading and shadowing. During some deep-fading, a wireless channel can be so bad that transmission can fail. The performance of wireless links is user location dependent. Especially in multicell IA as discussed in previous chapters, the remaining interference is dependent on the mobile users location which is the main factor that limits the achievable sum rates. Furthermore, the mobility of the users increases the variability of the transmission links. Such link variations require the user selection algorithms to be developed in order to deal with these time and location dependent challenges.

- Fairness: It is considered as a complicated issue for wireless networks. It may happen that a user is scheduled for transmission according to a certain service discipline or fairness guideline, which is independent of link state and the link is actually in error state [123]. If the user starts transmission, it will be corrupted and the transmission will waste the resources. In this case deferring transmission of this transmission until the link recovers from the errored state is clearly a reasonable choice. To determine fairness, two metrics are generally used. We will define these metrics in the following section, when we define the fair user selection algorithms.

- Quality of Service: Quality of Service (QoS) is the overall performance of a network. To quantitatively measure QoS, several metrics are considered such as error rates, bit rates, throughput, latency etc [123]. The wireless networks will provide services with various QoS requirements as transmission traffic is location and time dependent. Therefore QoS differentiation and guarantees must be supported. If a wireless link experiences frequent channel degradations, it is very difficult to guarantee QoS for the transmissions using this link. However, QoS should be guaranteed for the transmissions, either deterministically or statistically, on those links where the physical channel degradation does not exceed certain thresholds [123].

- Data Throughput and Channel Utilization: The bandwidth and therefore throughput is a very precious resource in wireless networks. An efficient user selection algorithm should aim to minimize inefficient transmission on error links and at the same time, maximize the utilization of the wireless channels [123]. 
- Power Constraint and Simplicity: Power constraints may be big concern in wireless communications. Especially, mobile users in uplink communications are power-constrained. Therefore they should be considered to design selection algorithms. Moreover, simplicity is also important so that power constraints can be executed at high speed to schedule real-time multimedia traffic with stringent timing requirements [123].

In order to guarantee a minimum transmission performance, as well as to provide varying QoS in the presence of channel fading and interference, power adaptation techniques are also necessary [124]. In this section, we also study some power adaptation techniques to improve the system capacity, following the user selection methods.

\subsection{User Selection Methods}

In this section, certain user selection algorithms are reviewed. We later analyse their performance for the multicell networks in the downlink which was considered in Chapter 6, Section 6.3.

\subsubsection{Fair User Selection Algorithms}

Fairness is a very important issue in user selection. However, there is a tradeoff between rate and fairness. To determine the fairness in the system, we use Jain's fairness metric [125]

$$
f_{\text {jains }}=\frac{\left(\sum_{i=1}^{K} \bar{R}_{\alpha, i}\right)^{2}}{K \sum_{i=1}^{K}\left(\bar{R}_{\alpha, i}^{2}\right)},
$$

where $K$ is the total number of users and $\bar{R}_{\alpha, i}$ is the mean rate of the $i$ th user in cell $\alpha$. The algorithms with $f_{\text {jains }}=1$ are defined as fair user algorithms. There are well known fair user algorithms in the literature, such as Round Robin (RR) and opportunistic Round Robin (ORR).

\section{Round Robin}

When a user connects to the BS, it is given a position in the queue of users and the scheduler will iterate through the queue.

\section{Opportunistic Round Robin}

The scheduler iterates the list of users and everytime the best user is selected and removed from the list. 


\subsubsection{Norm Based User Selection}

In norm-based user selection, users are selected based only upon their channel strengths. So $K$ users are sorted in descending order of their channel norms, and first $M_{\mathrm{ST}}$ users are selected for transmission [126]. For example, let us consider the received signal given in (5.23). As the BS has the knowledge of the desired channels, it select the users from the initial user set $\mathbb{A}_{0}=\{1, \ldots K\}$ with maximum channel norms

$$
\tilde{i}=\arg \max _{i \in \mathbb{A}_{0}}\left\|\mathbf{H}_{\alpha, i}\right\|_{F}
$$

where $\tilde{i} \in \tilde{\mathbb{A}}_{0}$ and $\tilde{\mathbb{A}}_{0}$ is the set of $M_{\mathrm{ST}}$ selected users.

\subsubsection{Semi-Orthogonal User Selection}

The user selection metric for Semi-Orthogonal user selection is the combination of the channel strength and its spatial orthogonality with respect to the other users [126-128]. According to this technique, the transmitter selects the first user from the initial user set $\mathbb{A}_{0}=\{1, \ldots K\}$ as

$$
\tilde{i}^{(1)}=\arg \max _{i \in \mathbb{A}_{0}}\left\|\mathbf{H}_{\alpha, i}\right\|_{F}
$$

After selecting $\tilde{i}^{(1)}$ user which is $\tilde{i}^{(1)} \in \mathbb{A}_{0}^{(1)}$, the next user, $\tilde{i}^{(2)} \in \mathbb{A}_{0}^{(2)}$ is selected within the user set as

$$
\tilde{i}^{(2)}=\left\{1 \leq i \leq K:\left\|\mathbf{H}_{\alpha, i} \mathbf{H}_{\alpha, j}^{*}\right\|_{F} \leq \tilde{\xi}\right\}
$$

where $j \in \mathbb{A}_{0}^{(1)}$ and $\tilde{\xi}$ is the design parameter which dictates the orthogonality allowed between channels. In this way, the transmitter can choose users that have high channel qualities and are mutually semi-orthogonal.

\subsubsection{SLNR-Based User Selection}

Based on this technique, the $\mathrm{BS}$ selects $M_{\mathrm{ST}}$ users which have the maximum SLNR ratio [129]

$$
\tilde{i}=\arg \max _{i \in \mathbb{A}_{0}} \operatorname{SLNR}_{\alpha, i}
$$

where $\mathrm{SLNR}_{\alpha, i}$ is defined in (5.37). For optimized transceivers, we will assume that $\overline{\mathbf{P}}$ is an identity matrix in (5.37) as there is no colourization used in optimized transceivers. 


\subsubsection{Estimated Rate User Selection}

In this selection technique, the BS orders the users based on their estimated rates. In our system of Section 6.3., the BS has the knowledge of the desired and intercell interference channels, however, it doesn't have the knowledge of intercell precoders. Therefore it can estimate the rate and order the users according to this information [3]. The users are selected as

$$
\tilde{i}=\arg \max _{i \in \mathbb{A}_{0}} \tilde{\bar{R}}_{\alpha, i}
$$

$\tilde{\bar{R}}_{\alpha, i}$ is the estimated rate for $i$ th user in cell $\alpha$ and can be obtained by [3]

$$
\tilde{\bar{R}}_{\alpha, i}=\log _{2}\left(1+\frac{\left(\frac{\rho_{\alpha, i}}{S}\right) \mathbf{u}_{\alpha, i}^{*} \mathbf{H}_{\alpha, i} \mathbf{v}_{\alpha, i} \mathbf{v}_{\alpha, i}^{*} \mathbf{H}_{\alpha, i}^{*} \mathbf{u}_{\alpha, i}}{\tilde{\bar{\Delta}}_{\alpha, i}+\Gamma_{\alpha, i}+\Lambda_{\alpha, i}}\right)
$$

where $\Gamma_{\alpha, i}$ is given in (6.63) and $\Lambda_{\alpha, i}$ is given in (6.64). Estimated dominant intercell interference, $\tilde{\bar{\Delta}}_{\alpha, i}$, can be computed as

$$
\tilde{\bar{\Delta}}_{\alpha, i}=\mathbf{u}_{\alpha, i}^{*} \sum_{\beta}\left(\frac{\rho_{\beta, i}}{N_{t}}\right) \mathbf{G}_{\beta, i} \mathbf{G}_{\beta, i}^{*} \mathbf{u}_{\alpha, i} .
$$

\subsubsection{Minimum Remaining Interference User Selection}

The criterion for this selection is the users which suffer the least remaining interference and selected according to

$$
\tilde{i}=\arg \min _{i \in \mathbb{A}_{0}} \operatorname{INR}_{\mathrm{rem}_{\alpha, i}} .
$$

\subsubsection{SLRINR-Based User Selection}

The user selection metric that this technique is based on is the maximum SLRINR ratio

$$
\tilde{i}=\arg \max _{i \in \mathbb{A}_{0}} \operatorname{SLRINR}_{\alpha, i}
$$

where $\operatorname{SLRINR}_{\alpha, i}$ is defined in (5.42). For optimized transceivers, we will assume that $\overline{\mathbf{P}}$ is an identity matrix in (5.42) as there is no colourization used in the optimized transceivers. 


\subsubsection{Simulation Results}

In this section, we give simulation results of some user selection techniques given in previous sections, considering the optimized transceivers proposed in Section 6.3. We consider there are 5 users in the network and the BS selects 3 users out of these 5 users. We first consider the channel estimation is perfect for direct and cross channels.

As seen in Fig. 7.1, the technique considering remaining interference only doesn't provide much gain over random user selection. However, when we consider norm of the desired channels, the gain that can be achieved with user selection is significant. We note that semi-orthogonal user selection scheme also provides as much gain as norm based user selection, however it doesn't provide much better sum rates as the number of users in the chosen set is not enough to observe the difference. We also show that SLRINR-based user selection and estimated rate user selection achieves the best ergodic mean sum rates. however the difference between these two techniques and both norm-based and semi orthogonal user selection is not significant. The reason is the fact that the optimized transceivers already reduce the effects of remaining interference.

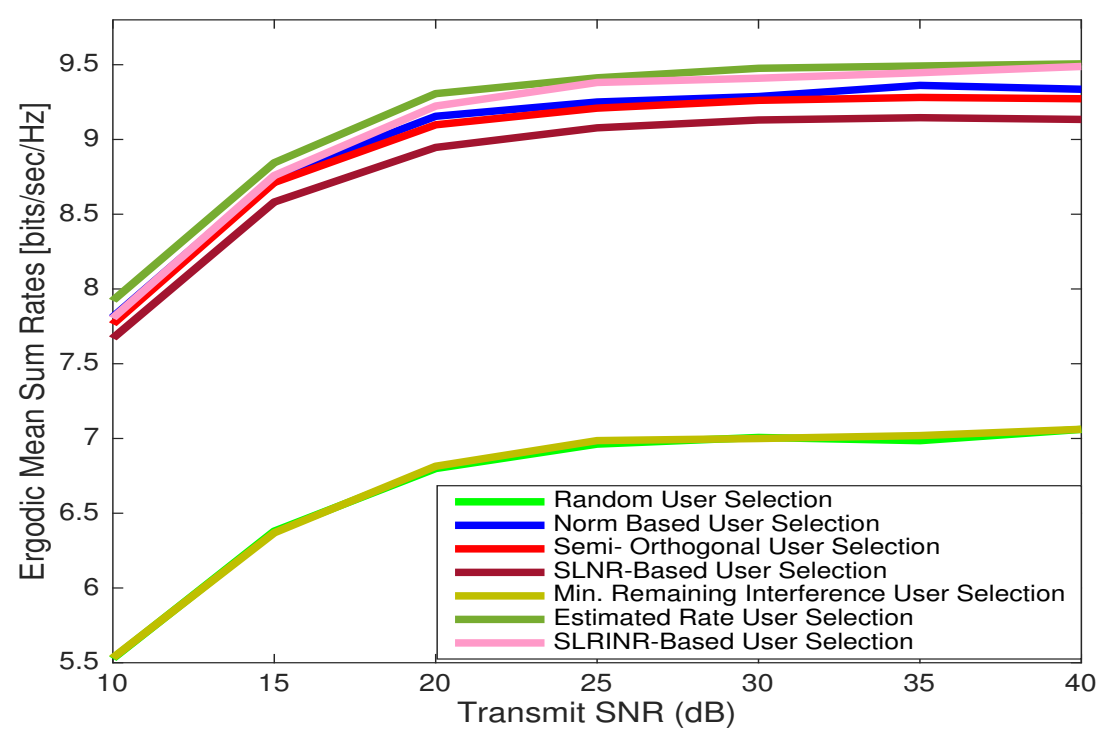

Figure 7.1: Ergodic mean sum rates of optimized transceivers with user selection methods for random user locations with perfect CSI and CCSI (Scenario 1 Downlink multicell systems) 


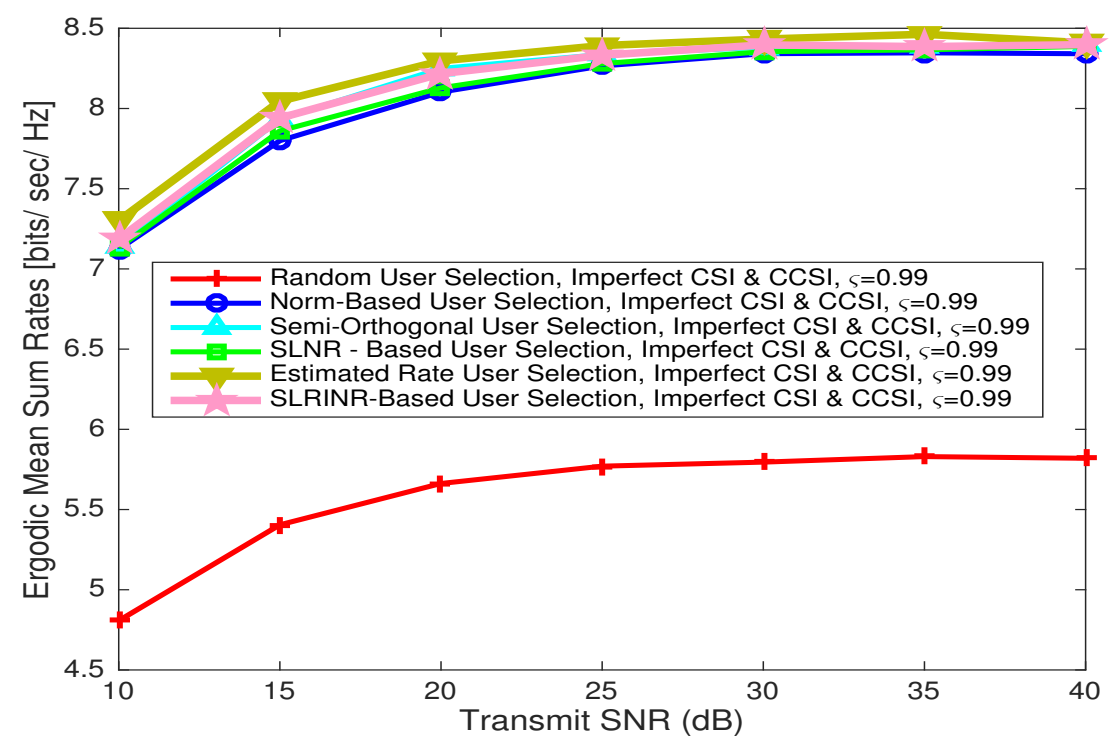

Figure 7.2: Ergodic mean sum rates of optimized transceivers with user selection methods for random user locations with imperfect CSI and CCSI (Scenario 1 Downlink multicell systems)

Later, we investigate how user selection techniques perform with channel imperfections. We first consider both links from desired users and interfering users are erroneous. We consider $\varsigma=0.99$ for both CSI and CCSI.

In Fig. 7.2, we note that Norm-based, Semi orthogonal-SLNR-SLRINR and estimated rate user selection techniques provide almost the same performance. We also observe that user selection provides approximately $25 \%$ gains over random user selection. This difference for perfect CSI and CCSI in Fig. 7.1 is 32\% for SLRINR based user selection and estimated rate user selection.

\subsection{Power Adaptation Methods}

Each user's signal may undergo independent fading and suffer different levels of interference power because the users may not be in the same location in a multicell environment. The users in a good channel condition may be allowed to transmit data as discussed in previous section. Another way of dealing with maximizing the throughput is the appropriately adapted transmit power for each user. Our contributions of this section are as follows

- We apply some power adaptation techniques given in [124] to our proposed system given in Section 6.3.

- We propose uniform power adaptation based on SLRINR.

First we define the power adaptation coefficient and rewrite the downlink received signal at user $k$ in cell $\alpha$ as given by 
$\mathbf{y}_{\alpha, k}=\left(\sqrt{\frac{\rho_{\alpha, k}}{S}}\right) \mathbf{H}_{\alpha, k} \sum_{i=1}^{K} \tilde{\mathbf{v}}_{\alpha, i} x_{\alpha, i}+\sum_{\beta}\left(\sqrt{\frac{\rho_{\beta, k}}{S}}\right) \mathbf{G}_{\beta, k} \sum_{l=1}^{K} \tilde{\mathbf{v}}_{\beta, l} x_{\beta, l}+\mathbf{z}_{\alpha, k}+\mathbf{n}_{\alpha, k}$,

where $\tilde{\mathbf{v}}_{\alpha, k}=\psi_{\alpha, k} \mathbf{v}_{\alpha, k}$ and $\psi_{\alpha, k}$ is the scalar power adaptation coefficient for user $k$ in cell $\alpha$. In the following sections, we describe how $\psi_{\alpha, k}$ can be found.

\subsubsection{Uniform Power Adaptation}

Uniform power adapation is basically dividing total power $\Psi_{\alpha}$ at the BS equally for each $K$ user [124]

$$
\psi_{\alpha, i}=\frac{\Psi_{\alpha}}{S}=\frac{S}{S}=1
$$

\subsubsection{Frobenius Norm Uniform Power Adaptation}

A measure of the quality of a particular channel is its Frobenius norm. Hence, in [15], the power available at the BS is divided among users based on their relative Frobenius norms. Therefore the power allocated to user $\mathrm{k}$ is given by [124]

$$
\psi_{\alpha, k}=\Psi_{\alpha} \frac{\left\|\mathbf{H}_{\alpha, k}\right\|_{F}}{\sum_{i=1}^{K}\left\|\mathbf{H}_{\alpha, k}\right\|_{F}}=S \frac{\left\|\mathbf{H}_{\alpha, k}\right\|_{F}}{\sum_{i=1}^{K}\left\|\mathbf{H}_{\alpha, k}\right\|_{F}} .
$$

\subsubsection{Uniform Power Adaptation based on Estimated Rate}

Here we compute $\psi_{\alpha, k}$ based on the estimated rate as it is assumed that the BS has the knowledge of intercell interfering channels and remaining interference. However, the BS is lack of the actual information of these interfering precoding vectors. The BS allocate the power for each user according to their estimated rate [124]

$$
\psi_{\alpha, k}=\Psi_{\alpha} \frac{\tilde{\bar{R}}_{\alpha, i}}{\sum_{j=1}^{K} \tilde{\bar{R}}_{\alpha, i}}=S \frac{\tilde{\bar{R}}_{\alpha, i}}{\sum_{j=1}^{K} \tilde{\bar{R}}_{\alpha, i}}
$$

where estimated rate, $\tilde{\bar{R}}_{\alpha, i}$ is given in (7.7). 


\subsubsection{Uniform Power Adaptation based on SLRINR}

Here we compute $\psi_{\alpha, i}$ based on the SLRINR ratio

$$
\psi_{\alpha, k}=\Psi_{\alpha} \frac{\operatorname{SLRINR}_{\alpha, i}}{\sum_{j=1}^{K_{\alpha}} \operatorname{SLRINR}_{\alpha, j}}=S \frac{\operatorname{SLRINR}_{\alpha, i}}{\sum_{j=1}^{K_{\alpha}} \operatorname{SLRINR}_{\alpha, j}},
$$

where $\mathrm{SLRINR}_{\alpha, i}$ is defined in (5.42). For optimized transceivers, we will assume that $\overline{\mathbf{P}}$ is an identity matrix in (5.42) as there is no colourization used in optimized transceivers.

\subsubsection{Simulation Results}

Here, we give simulation results of the power adaptation schemes given in this section, considering optimized transceivers given in Section 6.3 for downlink networks. We assume $N_{t}=N_{r}=4$ and there are 3 users in each cell. We consider cellular scenarios given in Chapter 2, Section 2.3.2., such as random user locations and cell-edge user locations.

\section{Random User Locations}

In Fig. 7.3, we give ergodic mean sum rate results for randomly located users. As seen in the figure, power adaptation slightly increases the ergodic mean sum rates, however this increase doesn't seem to be as significant as user selection techniques achieve.

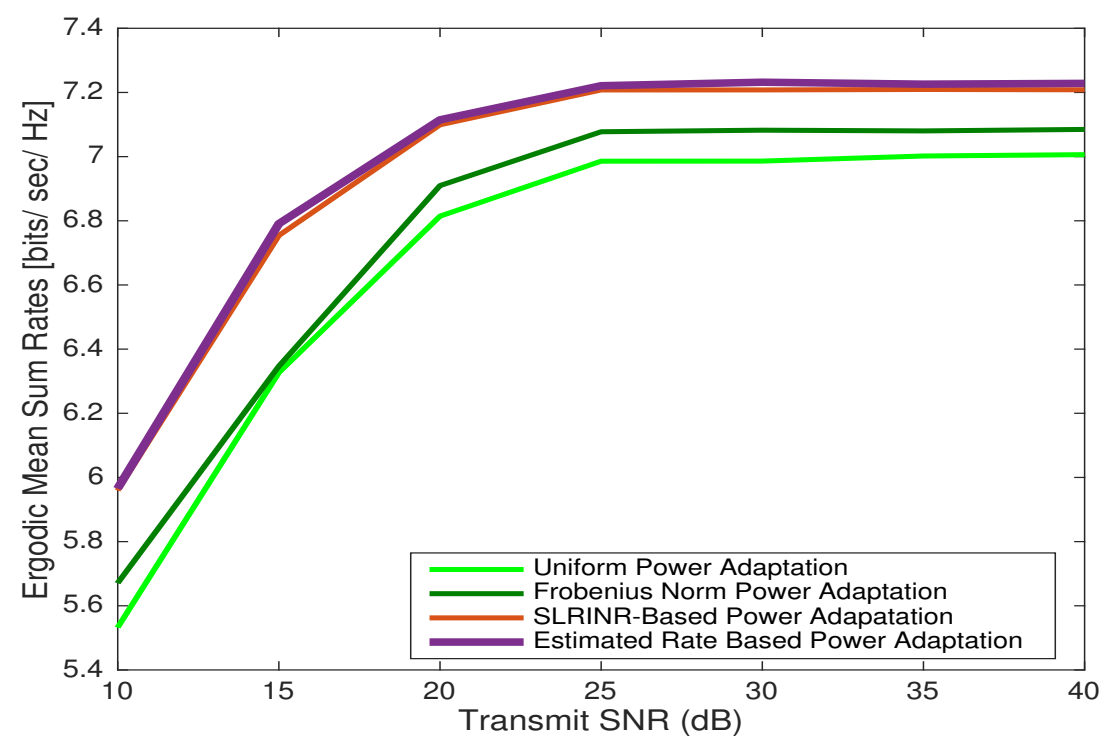

Figure 7.3: Ergodic mean sum rates of optimized transceivers with power adaptation for random user locations with perfect CSI \& CCSI (Scenario 1 - Downlink multicell systems) 
We observe in Figs. 7.3 that SLRINR and estimated rate power adaptation achieves the best ergodic mean sum rates, approximately $4 \%$ higher than uniform power adaptation.

Next, we give simulation results considering imperfect CSI \& CCSI in Fig. 7.4. We observe that estimated rate power adaptation achievs higher ergodic mean sum rates compared to other power adaptation techniques. All power adaptation techniques except estimated rate power adaptation achieve very minor gains, almost as same as uniform power adaptation. However, estimated rate power adaptation achieves approximately 3\% higher ergodic mean sum rates over uniform power adaptation.

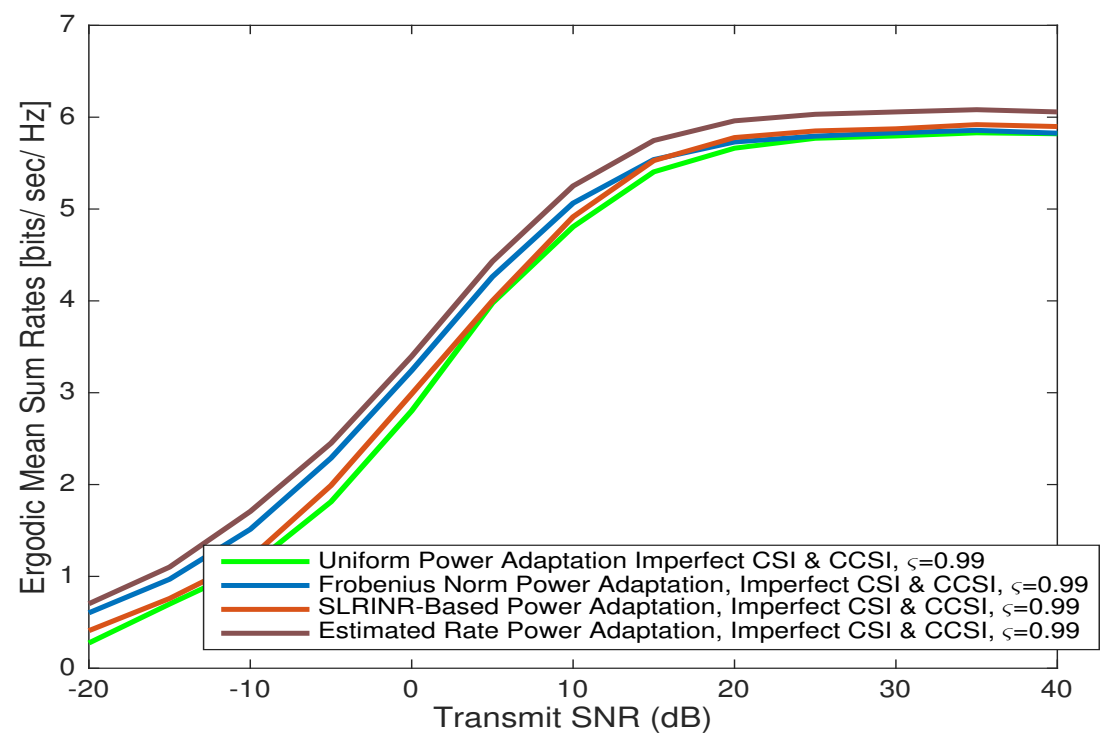

Figure 7.4: Ergodic mean sum rates of optimized transceivers with power adaptation for random user locations with imperfect CSI \& CCSI (Scenario 1 Downlink multicell systems)

\section{Cell-Edge User Locations}

Here, we give simulation results for cell edge users, considering 3GPP simulation results given in Chapter 2. In Fig. 7.5, we give ergodic mean sum rates for such users with assumption of perfect CSI and CCSI. Similar to random user locations, SLRINR-Based and estimated rate power adaptation techniques achieve the best ergodic mean sum rates. Estimated rate power adaptation provides approximately 15\% higher ergodic mean sum rates over uniform power adaptation. This gain is significantly higher than what power adaptation can achieve for randomly located users. Therefore, we observe that cell-edge users benefit from power adaptation more than the other users.

Finally, we analyse the cell-edge users with power adaptation techniques under the assumption of imperfect CSI and CCSI. We assume the imperfection 
parameter in $(2.23)$ and $(2.24), \varsigma=0.99$. We give ergodic mean sum rates of such users in Fig,. 7.6. We observe that estimated rate power adaptation again achieves the best ergodic mean sum rates, approximately $6 \%$ higher than what could be achieved with uniform power adaptation.

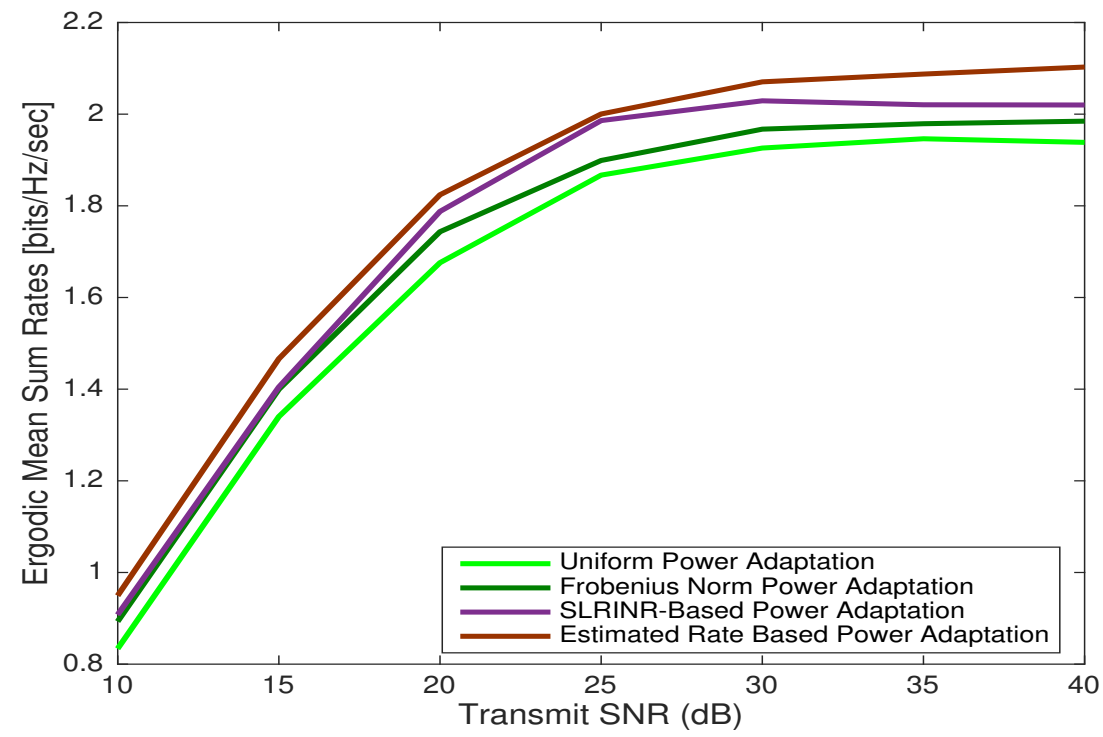

Figure 7.5: Ergodic mean sum rates of optimized transceivers with power adaptation for cell-edge user locations with perfect CSI \& CCSI (Scenario 2 - Downlink multicell systems)

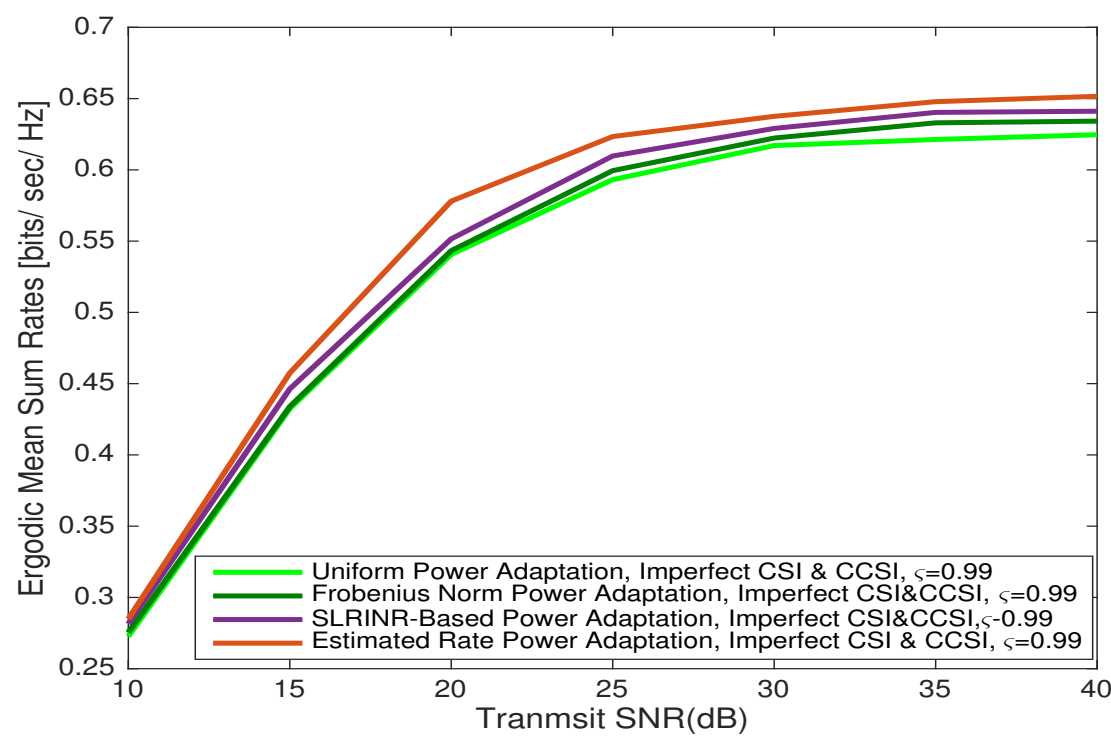

Figure 7.6: Ergodic mean sum rates of optimized transceivers with power adaptation for cell-edge user locations with imperfect CSI \& CCSI 


\section{User Selection and Power Adaptation Combined}

Finally, we give the simulation results to show the gain that can be achieved with considering user selection and power adaptation. Here, we compare estimated rate based power adaptation and also estimated rate based user selection with uniform power adaptation and random user selection. Again, we select 3 users out of 5 .

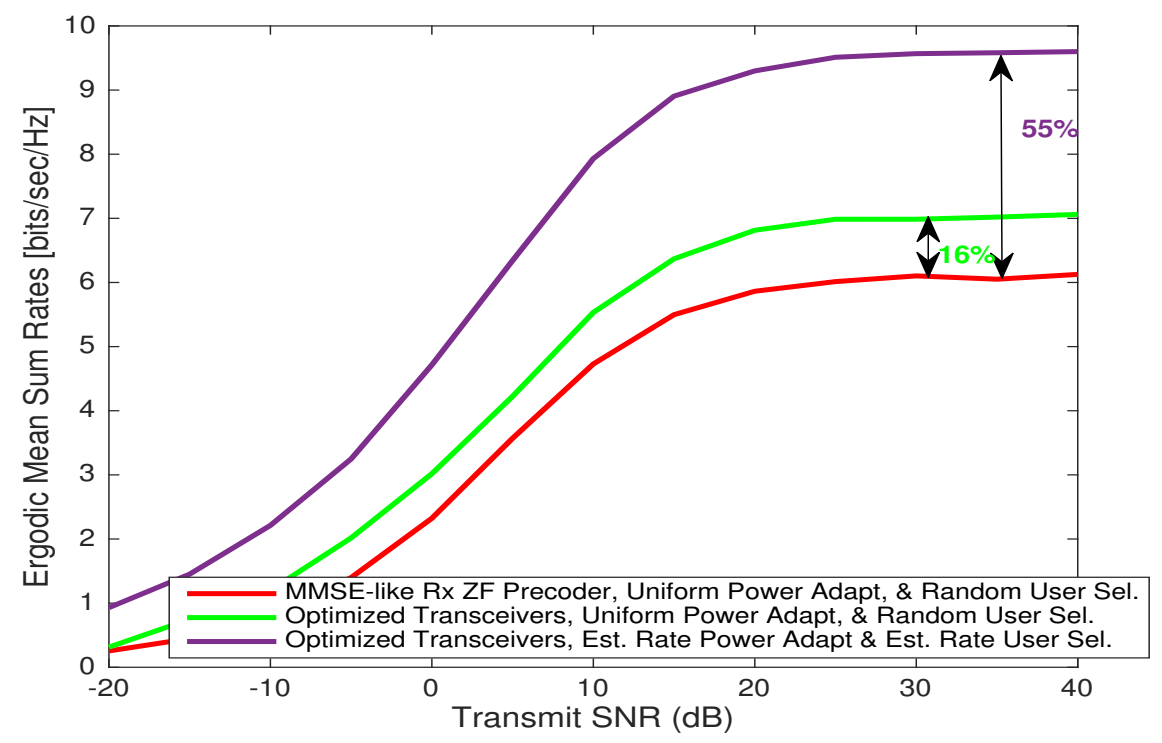

Figure 7.7: Improving the mean sum rates with user selection and power adaptation (Scenario 1 - Downlink multicell systems)

In Fig. 7.7, we show the gain that can be achieved with optimized transceivers and also with power adaptation and user selection. It is observed that the optimized transceivers of Section 6.3. can achieve approximately $16 \%$ better ergodic mean sum rates over MMSE-like receiver and ZF precoders of [3]. We show that implementing power adaptation and user selection based on estimated rates, the optimized transceivers can achieve approximately $55 \%$ better ergodic mean sum rates over the technique of [3].

\subsection{Summary}

Exponentially increasing user numbers and need for higher data rates forces the network providers to use the transmission resources very efficiently. Spectral efficiency can be improved with favouring the users which enjoy higher channel gains. This can be done with user selection and adapting the transmission power according to the user channel information.

In this chapter, we have studied some user selection and power adaptation techniques in order to improve the mean sum rates of a multicell network. We first 
implemented some user selection algorithms into a downlink multicell system employing our proposed optimized transceivers. We have shown that user selection based on estimated rate and SLRINR gives the best ergodic mean sum rates. We have shown simulation results with both perfect and imperfect channel knowledge. We have noted that selecting 3 users out of 5, based on their estimated rates, the ergodic mean sum rates could be improved by approximately $32 \%$ over randomly selecting the users.

Later, we have applied some power adaptation techniques. Here, we have observed that the gain that can be achieved by power adaptation is not as much as the gain that can be achieved with selecting the users based on their estimated rates. We have shown that the ergodic mean sum rates can be improved by $4 \%$ using estimated rate power adaptation for randomly located users. However, we have also noted that the cell-edge users benefit more from power adaptation, such that the ergodic mean sum rates can be improved approximately $15 \%$ over uniform power adaptation.

Finally we have shown the gain that can be achieved combining power adaptation and user selection based on estimated rates. We have shown that the optimized transceivers can achieve almost $55 \%$ better ergodic mean sum rates over the technique of [3], which is a very significant gain. 


\section{8}

\section{Conclusions and Future Work}

In this chapter, we present the conclusion of this research and also provide directions for future research work in this area.

\subsection{Conclusions}

In this thesis, we studied IA and IAC schemes in various wireless communication systems. First, we gave a technical background in Chapter 2. We defined fundamental terms for wireless systems. Later, we discussed cellular networks and provided simulation results for both the uplink and the downlink to show how much interference impacts the performance of the cellular networks. For example, intercell interference for randomly located users in a downlink network could cause $19 \mathrm{~dB}$ loss in median SINR. This is a very significant loss. On the other hand, we also mentioned in this chapter that traditional interference management systems could not provide an efficient solution, for example TDMA/FDMA could lead to inefficient usage of spectrum. The IA would enable users to share the same radio resources at the same time without any interference, therefore it would increase the efficiency of the spectrum. Interference could be aligned in a smaller subspace with carefully generated precoding vectors. In this chapter, we included some simple examples of the IA for MIMO $\mathrm{X}$ channels and interference channels. Here, we mentioned that the concept of the IA is dependent on some perfect assumptions therefore we explained the practicality challenges of IA.

In order to show the practicality issues of IA, we discussed an IA scheme in multihop interference channels where relays were employed in Chapter 3. We considered the scheme for $2 \times 2 \times 2$ interference channels which consisted of 2 
transmitter 2 relays and 2 receivers. Furthermore we assumed imperfect CSI which could cause misalignment of interference due to erroneous precoders. We derived analytical expressions for the SNR and SINR at the relays. The interference caused by misalignment due to the CSI errors was shown to be very prominent with the mean tending to infinity. We also noted that the interference due to the CSI errors could be modelled via simulation by a log normal distribution. In summary, aligned interference neutralisation scheme for multihop relay networks was very prone to channel estimation errors that accumulate over time. This leads us to conclude that the gain proposed by the $2 \times 2 \times 2$ channel could only be achievable in an ideal setting. The ZF receivers also contribute further to noise enhancement. Therefore, the results of Chapter 3 motivated us to investigate how we could design precoding vectors which could perform better with imperfect CSI. Furthermore, we decided to look at different kind of receivers instead of ZF receivers in order to avoid noise and interference enhancement.

Having enough dimensions plays an important role in aligning all interference within a subspace together. This is especially very important for the multicell case where there are multiple users served simultaneously. The multiple users sharing the same radio resources at the same time interfere with each other, degrading the overall system performance. Intuitively, the more interfering signals that need to be aligned, the larger the number of dimensions needed to align them. In Chapter 4, we mainly addressed how to apply IA schemes in the uplink. We started discussing the schemes with two cell case for each type of network and then we explained how to deal with large networks where there are many cells serving many users. We showed how much imperfect channel estimation could impact on the overall system performance. Besides dependency on perfect CSI, we also discussed the dimensionality challenge. The existing technique studied for two-cell case could be problematic for networks which consists more than two-cell. As the size of the network gets bigger, we need to exploit more dimensions at the transmitter and the receiver. This would result in employing many antennas, however considering the size of the mobile handsets, it is almost impossible to have so many antennas to align all interference at the transmitter side. Motivated by this, we later considered a multicell network in the uplink, where there are more than two cells in the network with many users being served in each cell. We discussed about cancelling the interference with existing receiver architectures and addressed the challenges that we encounter with ZF and MMSE postcoding vectors. The ZF receiver has a requirement of having enough number of dimensions to cancel the interference. Furthermore, it could enhance the noise and interference. On the other hand, the MMSE receiver needs the knowledge of out-of-cell precoding vectors which is very difficult to obtain in practical systems. Here, we categorized the interference as 
dominant interference and remaining interference and we proposed a novel receiver called the combined receiver which is a combination of ZF and MMSE postcoders. According to this scheme, the receivers could cancel the dominant interference with the ZF part, whereas reduce the remaining interference with MMSE part. We demonstrated that our proposed combined receiver could achieve significantly better mean sum rates compared to the existing receivers. We also considered antenna correlation as in (2.25) because the combined receiver may require many antennas to be employed at the BS, depending on the number of interferers to be ZF'd. However, as the combined receivers leave the remaining interference to be handled with the MMSE part, it requires less number of antennas at the receivers compared to ZF postcoding vectors.

After discussing the schemes for the uplink, we discussed downlink IAC techniques in Chapter 5. We first gave an IA scheme for a 2-cell downlink system. We reproduced and discussed the results of [111] but also considering imperfect CSI. Then we analysed a scheme that was proposed in [3] for cellular systems where there are more than two cells. We reproduced and expanded the results of [3] with their proposed MMSE-like postcoding vectors and ZF precoding vectors. Authors of [3] found the colouring parameter heuristically. In Chapter 5 , we applied an analytical approach to find optimum colouring parameter to improve the mean sum rates. Furthermore, we applied SLNR-based precoding vectors which perform better for low-transmit SNR regions and they achieve approximately same mean sum rates as ZF precoding vectors at high transmit SNR. We also proposed a new precoding vector based on SLRINR. As it considers the remaining interference, it provides significantly higher mean sum rates compared to other existing precoding schemes at all transmit SNRs. For example, we showed that cell-edge users could achieve approximately $45 \%$ higher mean sum rates than what ZF and SLNR could achieve. However, we also showed that this gain is susceptible to perfect channel knowledge.

We noted that little estimation error in the CCSI $(\varsigma=0.99)$ could result in significant performance degradation. However, SLRINR precoders could still achieve $28 \%$ more mean sum rates than ZF and SLNR precoders with imperfect CCSI. This gain with imperfect CCSI motivated us to investigate different levels of cooperation between adjacent cells. Thus in Chapter 5, we investigated a partial cooperative interference cancellation scheme for a downlink cellular network. We proposed that the cooperative users in the cooperation zone use the MMSE-like receivers and could cancel intercell interference using CCSI from neighbouring cells. Other users located outside the cooperation zone were assumed to use the MF receivers. We showed that the system could achieve better sum rates with cooperation, provided there is reasonable CCSI accuracy. Setting the cooperation threshold as $8 \mathrm{~dB}$, we demonstrated that the partial cooperative scheme can achieve gains of approximately $25 \%$ in mean sum rates relative to 
the no-cooperation scheme at a transmit SNR of $40 \mathrm{~dB}$. We considered urban and suburban environments and computed the ratio of the users falling in the overlapping regions (i.e. subject to cooperation) for urban and suburban environments. We compared the results for ZF, SLNR and SLRINR-based precoders and showed that SLNR precoders achieve better sum rates than ZF precoders at low transmit SNRs while their performance is equivalent to ZF precoders at high transmit SNRs. However, we also demonstrated that the transmit SNR value at which the performance of SLNR precoders approaches that of ZF precoders is different for different environments. For suburban users, this value is higher than for urban areas, because of lower interference levels. We also considered imperfect CCSI between the cooperative users and interfering BSs. We also provided an analytical expression for the additional interference caused by imperfect CCSI. We confirmed the accuracy of this analytical expression via simulation results which show that the analytical expression perfectly matches with the simulation results. We also noted that the additional interference for urban users is more than for suburban users as a result of path loss differences. The techniques proposed in Chapter 5 were not optimum solutions. Moreover, they would require many iterations between users and the BSs which would increase the overhead. Therefore in Chapter 6 , we investigated the jointly optimisation of IAC techniques for both uplink and downlink multicell networks. First, we proposed novel optimised transceivers for the uplink. We derived optimum precoder-postcoder set that minimizes the total MSE at the BS. We showed that optimised transceivers could achieve significantly better results compared to the scheme of combined receivers with ZF precoders [1], which we proposed in Chapter 4. We also considered implementing the combined receivers in the optimisation. We demonstrated that optimised transceivers with combined receivers perform well, however, not better than the optimised transceivers with MMSE receivers, because the ZF part of the combined receiver is enhancing the remaining interference and noise. However it could still provide higher ergodic mean-sum rates compared to non-optimised transceivers, the MMSEreceiver with $\mathrm{ZF}$ precoders and the combined receiver with $\mathrm{ZF}$ precoders. We also considered MMSE receivers with SLRINR precoders. The simulation results indicated that the optimised transceivers could also outperform MMSE receivers with SLRINR precoders, at high transmit SNRs. Furthermore, we considered cell-edge users only, using the 3GPP scenarios given in Chapter 2. As cell-edge users suffer from interference drastically, compared to other users, the gain that can be achieved with optimised transceivers is more prominent. Optimised transceivers with MMSE receivers were shown to achieve significant gains over MMSE receivers with ZF precoders.

After the uplink, we also proposed novel optimized transceivers for the downlink. We demonstrated that optimized transceivers could achieve significantly 
better mean sum rates compared to the existing scheme of [3]. For example, considering 2 dominant interference, the proposed optimized transceivers could achieve $16 \%$ better mean sum rates over the technique of [3]. As cell-edge users suffer interference more than other users, the proposed optimized transceivers could achieve $67 \%$ higher mean sum rates than [3].

Spectral efficiency could also be improved more with user selection and/or adapting the transmission power according to the user channel information. Thus in Chapter 7, we studied some user selection and power adaptation techniques. We first implemented some user selection algorithms into a downlink multicell system employing our proposed optimised transceivers. We demonstrated that user selection based on estimated rate and SLRINR gives the best ergodic mean sum rates. We also provided simulation results with both perfect and imperfect channel knowledge. We noted that selecting 3 users out of 5 users, based on their estimated rates, the ergodic mean sum rates could be improved by approximately $32 \%$ over randomly selecting the users. Later, we applied some power adaptation techniques. Here, we observed that the gain that could be achieved by power adaptation is not as much as the gain that could be achieved with selecting the users based on their estimated rates. Finally we demonstrated the gain that could be achieved combining power adaptation and user selection schemes based on estimated rates. The simulation results indicated that the optimised transceivers could achieve almost $55 \%$ better ergodic mean sum rates for randomly located users over the technique of [3], which is a very significant gain.

\subsection{Future Work}

In this section, some suggestions for future work are presented

\subsubsection{Analytical Analysis of System Performance Metrics with Imperfect CSI and CCSI}

Performance analysis is a decisive prerequisite for the system design, therefore it presents appropriate information for performance optimisation [130,131]. In previous chapters, we have shown that CSI at the transmitters and receivers has a great impact on the performance of IA and IAC schemes. We derived analytical expressions for the SNR and SINR for a multihop relay network in [99]. In $[132,133]$, the authors analysed the average residual interference caused by imperfect IA due to the CSI exchange amount. The authors of [134] derived closed-form expressions of outage probability, ergodic rate and symbol error rates for a single-cell multi-user IA scheme. We also analytically derived the additional interference caused by imperfect CCSI for IAC scheme in a multicell 
downlink network in [109]. However, a comprehensive analytical analysis of some system performance metrics, such as outage probability, ergodic rates, for IA and IAC schemes in multicell networks with imperfect CSI and CCSI is still unsolved.

For a complete analytical analysis for our proposed systems with imperfect CSI and CCSI, such as MMSE-like receivers with SLRINR precoders and optimised transceivers, the performance metrics should be derived analytically with respect to imperfect channel estimation parameters given in (2.23) and (2.24). However it is a complex task to complete and requires the knowledge of the following

- The pdfs of SLRINR precoders and optimised transceivers are very difficult to derive analytically as they both involve maximum eigenvector of a matrix. Due to our best knowledge, statistical characterisation of the maximum eigenvector of only Wishart distribution is currently available $[135,136]$ and the matrices we worked on did not have Wishart distribution. As a future work, it is very important to find the distribution of the maximum eigenvector with which we generate our proposed precoding vectors.

Also, the design of optimised transceivers consist of many iterations which make it very challenging to derive closed-form expressions for system metrics. However, closed-form expressions of the system metrics for combined receivers with ZF precoders are much easier to derive as they do not consist of any maximum eigenvector or iteration. Similarly, IA schemes for 2 cell networks can be analyzed analytically and improved for more robust systems which are less susceptible to channel imperfections.

\subsubsection{IAC in Next Generation Wireless Communications}

The fifth generation ( $5 \mathrm{G}$ ) systems are aimed to be able to accommodate the ever increasing number of users who require ubiquitous access to high volumes of wireless data [137-139]. It is expected that 5G will provide up to 100 times higher number of simultaneously connected devices, compared to current systems. 5G technology is currently being considered for use in 28 and $36 \mathrm{GHz}$ bands $[137,140,141]$. These frequency bands are referred to as the millimetre bands (mm-band). Consequently, 5G communication is often termed as millimetre-wave (mmWave) communication. The mmWave offers the potential to support gigabit-per-second data rates due to both the vast bandwidth available in mmWave bands and the use of the large number of antenna arrays [140]. The authors of [142] forecast one of the major challenges in the realization of mmWave systems as the use of a hybrid MIMO processor that consists of an analog beamformer and a digital beamformer jointly to compensate excessive 
path loss. This new new hybrid physical-layer aspect would change the architecture of the channel estimation and feedback. In [143], the authors showed that mmWave networks can improve on the performance and efficiency when considering both line-of-sight (LOS) and non-line-of-sight (NLOS) communication. Massive improvements in transmission capacity and area spectral efficiency (e.g. 10-100x) are possible when only communicating over LOS links which motivates LOS aware protocols [143]. Further, the authors of [143] showed the NLOS interference is negligible and LOS interference can still be the limiting factor for a mmWave ad hoc network. This also motivates the need for LOS interference alignment strategies. The authors of [144] demonstrated that the interference management in multi-user mmWave systems is required, even when the system is employed with large antenna arrays. They proposed a hybrid precoding scheme which involves a combination of analog and digital processing. However, they didn't take the intercell interference into consideration. Therefore it would be beneficial to develop a precoding scheme for multicellular mmWave communications which could also consider the intercell interference. In $[145,146]$, it is shown that linear receivers such as ZF and MMSE receivers could be employed in mmWave communications. Therefore, it would be beneficial to investigate the use of combined receivers for cancelling intercell interference in mmWave communications. Furthermore, optimisation of such systems considering hybrid precoding can be considered as a future work. 
Appendices 



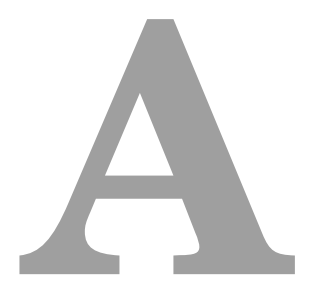

\section{Detailed explanation of (3.20)}

In this appendix, we show the derivation of (3.20). Considering the received signals (3.7) and (3.9)

$$
\mathbf{y}_{R_{1}}=\mathbf{H}_{11} \mathbf{v}_{1,1} x_{1,1}+\mathbf{H}_{11} \mathbf{v}_{2,1}\left(x_{1,2}+x_{2}\right)+\mathbf{n}_{1}^{[1]},
$$

and then the post-processed signal in (3.11),

$$
\mathbf{x}_{R_{1}}=\mathbf{H}_{R_{1}}^{-1} \mathbf{y}_{R_{1}}=\left[\begin{array}{c}
x_{1,1} \\
x_{1,2}+x_{2}
\end{array}\right]+\mathbf{H}_{R_{1}}^{-1} \mathbf{n}_{1}^{[1]} .
$$

Then, we take the expectation of $\mathbb{E}\left[\mathbf{x}_{R_{1}} \mathbf{x}_{R_{1}}^{*}\right]$ as in (3.19)

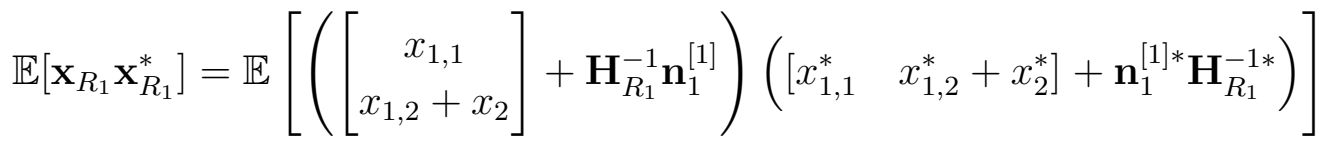

$$
\begin{aligned}
& {\left[\begin{array}{cc}
\mathbb{E}\left|x_{1,1}\right|^{2} & 0 \\
0 & \mathbb{E}\left|x_{1,2}\right|^{2}+\mathbb{E}\left|x_{2}\right|^{2}
\end{array}\right]+\mathbb{E}\left[\mathbf{H}_{R_{1}}^{-1} \mathbf{n}_{1}^{[1]} \mathbf{n}_{1}^{[1] *} \mathbf{H}_{R_{1}}^{-1 *}\right]}
\end{aligned}
$$

Assuming that $\mathbb{E}\left[x_{i j} x_{k l}\right]=0$ for $(i, j) \neq(k, l)$. Then we can rewrite (A.4) as

$$
\mathbb{E}\left[\mathbf{x}_{R_{1}} \mathbf{x}_{R_{1}}^{*}\right]=\quad\left[\begin{array}{cc}
\mathbb{E}\left|x_{1,1}\right|^{2} & 0 \\
0 & \mathbb{E}\left|x_{1,2}\right|^{2}+\mathbb{E}\left|x_{2}\right|^{2}
\end{array}\right]+\sigma^{2} \mathbb{E}\left[\mathbf{H}_{R_{1}}^{-1} \mathbf{H}_{R_{1}}^{-1 *}\right]
$$

The SNR can be known if we know $\mathbb{E}\left[\mathbf{H}_{R_{1}}^{-1} \mathbf{H}_{R_{1}}^{-1 *}\right]$ in (A.5). We give the definition 
of $\mathbf{H}_{\mathbf{R}_{\mathbf{1}}}$ after (3.12) in Chapter 3 as follows

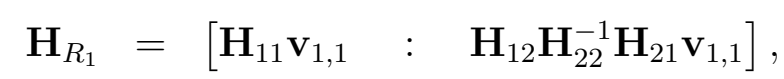

since $\mathbf{v}_{1,2}=\mathbf{H}_{11}^{-1} \mathbf{H}_{12} \mathbf{v}_{2,1}, \mathbf{v}_{2}=\mathbf{H}_{22}^{-1} \mathbf{H}_{21} \mathbf{v}_{1,1}$ and so that $\mathbf{v}_{1,2}=\mathbf{H}_{11}^{-1} \mathbf{H}_{12} \mathbf{H}_{22}^{-1} \mathbf{H}_{21} \mathbf{v}_{1,1}$. Using (A.6), we can rewrite $\mathbf{H}_{R_{1}}$ as follows

$$
\mathbf{H}_{R_{1}}=\left[\begin{array}{cc}
\frac{1}{h_{22}(1)} & 0 \\
0 & \frac{1}{h_{22}(2)}
\end{array}\right]\left[\begin{array}{ll}
h_{11}(1) h_{22}(1) & h_{12}(1) h_{21}(1) \\
h_{11}(2) h_{22}(2) & h_{12}(2) h_{21}(2)
\end{array}\right]
$$

Then, we can take the inverse of (A.7) as follows

$$
\mathbf{H}_{R_{1}}^{-1}=\frac{\mathbf{D}_{R_{1}}}{\Delta}
$$

where

$$
\begin{aligned}
\mathbf{D}_{R_{1}} & =\left[\begin{array}{cc}
h_{12}(2) h_{21}(2) & -h_{12}(1) h_{21}(1) \\
-h_{11}(2) h_{22}(2) & h_{11}(1) h_{22}(1)
\end{array}\right]\left[\begin{array}{cc}
h_{22}(1) & 0 \\
0 & h_{22}(2)
\end{array}\right] \\
& =\left[\begin{array}{cc}
h_{12}(2) h_{21}(2) h_{22}(1) & -h_{12}(1) h_{21}(1) h_{22}(2) \\
-h_{11}(2) h_{22}(2) h_{22}(1) & h_{11}(1) h_{22}(1) h_{22}(2)
\end{array}\right],
\end{aligned}
$$

and

$$
\Delta=h_{11}(1) h_{22}(1) h_{12}(2) h_{21}(2)-h_{12}(1) h_{21}(1) h_{11}(2) h_{22}(2)
$$

As previously mentioned, we need to know $\left[\mathbf{H}_{R_{1}}^{-1} \mathbf{H}_{R_{1}}^{-1 *}\right]$ which can be given as

$$
\begin{aligned}
{\left[\mathbf{H}_{R_{1}}^{-1} \mathbf{H}_{R_{1}}^{-1 *}\right] } & =\frac{1}{|\Delta|^{2}} \\
& {\left[\begin{array}{cc}
h_{12}(2) h_{21}(2) h_{22}(1) & -h_{12}(1) h_{21}(1) h_{22}(2) \\
-h_{11}(2) h_{22}(2) h_{22}(1) & h_{11}(1) h_{22}(1) h_{22}(2)
\end{array}\right] } \\
& {\left[\begin{array}{cc}
h_{12}^{*}(2) h_{21}^{*}(2) h_{22}^{*}(1) & -h_{11}^{*}(2) h_{22}^{*}(2) h_{22}^{*}(1) \\
-h_{12}^{*}(1) h_{21}^{*}(1) h_{22}^{*}(2) & h_{11}^{*}(1) h_{22}^{*}(1) h_{22}^{*}(2)
\end{array}\right] }
\end{aligned}
$$

The SNR of the first symbol can be written as given in (3.20) where $\Delta_{1}$ is the first element of (A.12) which is given as

$$
\Delta_{1}=\left|h_{21}(2)\right|^{2}\left|h_{12}(2)\right|^{2}\left|h_{22}(1)\right|^{2}+\left|h_{21}(1)\right|^{2}\left|h_{12}(1)\right|^{2}\left|h_{22}(2)\right|^{2},
$$




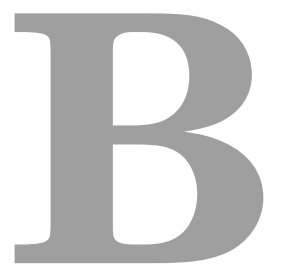

\section{Detailed Explanation of (6.7)}

In this appendix, we demonstrate the derivation of (6.7). To do this, we first write the equation for the MSE for each user as follows

$$
\mathrm{MSE}_{\alpha, i}=\mathbb{E}\left[\left|\tilde{x}_{\alpha, i}-x_{\alpha, i}\right|^{2}\right]
$$

where $\tilde{x}_{\alpha, i}$ is given in (6.2) and $x_{\alpha, i}$ is the transmitted signal for user $i$ in cell $\alpha$. Considering $\mathbf{u}_{\alpha, i}$ in (6.5) and $\mathbf{T}_{\alpha}$ given in (6.6), we can express MSE for user $i$ in cell $\alpha$ as follows

$$
\begin{aligned}
\operatorname{MSE}_{\alpha, i} & =\mathbb{E}\left[\left|\tilde{x}_{\alpha, i}-x_{\alpha, i}\right|^{2}\right] \\
& =\operatorname{tr}\left\{\Omega_{\alpha, i}\right\}
\end{aligned}
$$

where

$$
\begin{aligned}
\Omega_{\alpha, i} & =\mathbf{u}_{\alpha, i}^{*}\left(\sum_{j=1}^{K}\left(\left(\frac{\rho_{\alpha, j}}{S}\right) \mathbf{H}_{\alpha, j} \mathbf{v}_{\alpha, j} \mathbf{v}_{\alpha, j}^{*} \mathbf{H}_{\alpha, j}^{*}\right)+\left(\sum_{\beta} \sum_{j=1}^{K} \frac{\rho_{\beta, j}}{S} \mathbf{G}_{\beta, j}\left(\frac{1}{N_{t}} \mathbf{I}\right) \mathbf{G}_{\beta, j}^{*}\right)\right) \mathbf{u}_{\alpha, i} \\
& -\mathbf{v}_{\alpha, i}^{*} \mathbf{H}_{\alpha, i}^{*} \mathbf{u}_{\alpha, i} \sqrt{\left(\frac{\rho_{\alpha, i}}{S}\right)}-\sqrt{\left(\frac{\rho_{\alpha, i}}{S}\right)} \mathbf{u}_{\alpha, i}^{*} \mathbf{H}_{\alpha, i} \mathbf{v}_{\alpha, i}+1+\left(1+\mathrm{INR}_{\mathrm{rem}}\right) \mathbf{u}_{\alpha, i}^{*} \mathbf{I u}_{\alpha, i}
\end{aligned}
$$

Substituting (6.5) into (B.3), we get, 


$$
\begin{aligned}
& \Omega_{\alpha, i}=\mathbf{v}_{\alpha, i}^{*} \mathbf{H}_{\alpha, i}^{*}\left(\sqrt{\frac{\rho_{\alpha, i}}{S}}\right) \mathbf{T}_{\alpha}^{-1} \\
& \left(\sum_{j=1}^{K}\left(\frac{\rho_{\alpha, j}}{S}\right) \mathbf{H}_{\alpha, j} \mathbf{v}_{\alpha, j} \mathbf{v}_{\alpha, j}^{*} \mathbf{H}_{\alpha, j}^{*}+\left(\sum_{\beta} \sum_{j=1}^{K} \frac{\rho_{\beta, j}}{S} \mathbf{G}_{\beta, j}\left(\frac{1}{N_{t}} \mathbf{I}\right) \mathbf{G}_{\beta, j}^{*}\right)\right) \\
& \mathbf{T}_{\alpha}^{-1}\left(\sqrt{\frac{\rho_{\alpha, i}}{S}}\right) \mathbf{H}_{\alpha, i} \mathbf{v}_{\alpha, i}-\mathbf{v}_{\alpha, i}^{*} \mathbf{H}_{\alpha, i}^{*} \sqrt{\left(\frac{\rho_{\alpha, i}}{S}\right)} \mathbf{T}_{\alpha}^{-1} \sqrt{\left(\frac{\rho_{\alpha, i}}{S}\right)} \mathbf{H}_{\alpha, i} \mathbf{v}_{\alpha, i} \\
& -\mathbf{v}_{\alpha, i}^{*} \mathbf{H}_{\alpha, i}^{*} \sqrt{\left(\frac{\rho_{\alpha, i}}{S}\right)} \mathbf{T}_{\alpha}^{-1} \sqrt{\left(\frac{\rho_{\alpha, i}}{S}\right)} \mathbf{H}_{\alpha, i} \mathbf{v}_{\alpha, i} \\
& +1+\left(1+\mathrm{INR}_{\mathrm{rem}}\right) \mathbf{v}_{\alpha, i}^{*} \mathbf{H}_{\alpha, i}^{*} \sqrt{\left(\frac{\rho_{\alpha, i}}{S}\right)} \mathbf{T}_{\alpha}^{-1} \mathbf{T}_{\alpha}^{-1} \sqrt{\left(\frac{\rho_{\alpha, i}}{S}\right)} \mathbf{H}_{\alpha, i} \mathbf{v}_{\alpha, i} .
\end{aligned}
$$

Considering (6.6), we can write $\sum_{j=1}^{K} \frac{\rho_{\alpha, j}}{S} \mathbf{H}_{\alpha, j} \mathbf{v}_{\alpha, j} \mathbf{v}_{\alpha, j}^{*} \mathbf{H}_{\alpha, j}+\left(\sum_{\beta} \sum_{j=1}^{K} \frac{\rho_{\beta, j}}{S} \mathbf{G}_{\beta, j}\left(\frac{1}{N_{t}} \mathbf{I}\right) \mathbf{G}_{\beta, j}^{*}\right)=$ $\mathbf{T}-\left(1+\mathrm{INR}_{\text {rem }}\right) \mathbf{I}$, and we can rewrite (B.2)

$$
\begin{aligned}
\mathrm{MSE}_{\alpha, i} & =\operatorname{tr}\left\{\mathbf{v}_{\alpha, i}^{*} \mathbf{H}_{\alpha, i}^{*}\left(\sqrt{\frac{\rho_{\alpha, i}}{S}}\right) \mathbf{T}_{\alpha}^{-1}\left(\mathbf{T}-\left(1+\mathrm{INR}_{\mathrm{rem}}\right) \mathbf{I}\right) \mathbf{T}_{\alpha}^{-1}\left(\sqrt{\frac{\rho_{\alpha, i}}{S}}\right) \mathbf{H}_{\alpha, i} \mathbf{v}_{\alpha, i}\right. \\
& -\mathbf{v}_{\alpha, i}^{*} \mathbf{H}_{\alpha, i}^{*} \sqrt{\left(\frac{\rho_{\alpha, i}}{S}\right)} \mathbf{T}_{\alpha}^{-1} \sqrt{\left(\frac{\rho_{\alpha, i}}{S}\right)} \mathbf{H}_{\alpha, i} \mathbf{v}_{\alpha, i}-\mathbf{v}_{\alpha, i}^{*} \mathbf{H}_{\alpha, i}^{*} \sqrt{\left(\frac{\rho_{\alpha, i}}{S}\right)} \mathbf{T}_{\alpha}^{-1} \sqrt{\left(\frac{\rho_{\alpha, i}}{S}\right)} \mathbf{H}_{\alpha, i} \mathbf{v}_{\alpha, i} \\
& \left.+1+\left(1+\mathrm{INR}_{\mathrm{rem}}\right) \mathbf{v}_{\alpha, i}^{*} \mathbf{H}_{\alpha, i}^{*} \sqrt{\left(\frac{\rho_{\alpha, i}}{S}\right)} \mathbf{T}_{\alpha}^{-1} \mathbf{T}_{\alpha}^{-1} \sqrt{\left(\frac{\rho_{\alpha, i}}{S}\right)} \mathbf{H}_{\alpha, i} \mathbf{v}_{\alpha, i}\right\} .
\end{aligned}
$$

We note that $\mathbb{E}\left[x_{\alpha, i} x_{\alpha, k}\right]=0$, where $i \neq k$ as the symbols transmitted from the BS are i.i.d. Therefore, the result in (B.5) can be simplified to

$$
\mathrm{MSE}_{\alpha, i}=\left(1-\mathbf{v}_{\alpha, i}^{*} \mathbf{H}_{\alpha, i}^{*} \mathbf{T}_{\alpha}^{-1}\left(\frac{\rho_{\alpha, i}}{S}\right) \mathbf{H}_{\alpha, i} \mathbf{v}_{\alpha, i}\right)
$$

which is given in (6.7). 


\section{Bibliography}

[1] R. F. Ustok, P. Dmochowski, P. Smith, and M. Shafi, "Interference alignment with combined receivers for heterogeneous networks," in Proceedings of IEEE International Conference on Communications, pp. 5287 -5292, 2014.

[2] T. Gou, S. Jafar, and S.-Y. Chung, "Aligned interference neutralisation and the degrees of freedom of the $2 \times 2 \times 2$ interference channel," IEEE Transactions on Information Theory, vol. 58(7), pp. 4381-4395, 2012.

[3] C. Suh, M. Ho, and D. Tse, "Downlink interference alignment," IEEE Transactions on Information Theory, vol. 59(9), pp. 2616-2626, 2011.

[4] D. Gesbert, a. H. H. Hanly, S., S. Shamai Shitz, O. Simeone, and W. Yu, "Multi-cell MIMO cooperative networks: A new look at interference," IEEE Journal on Selected Areas in Communications, vol. 28(9), pp. 13801408, 2010.

[5] D. Gesbert, S. Kiani, A. Gjendemsj, and G. Oien, "Adaptation, coordination, and distributed resource allocation in interference-limited wireless networks," Proceedings of the IEEE, vol. 95(12), pp. 2393-2409, 2007.

[6] A. Carleial, "Interference channels," IEEE Transactions on Information Theory, vol. 24(1), pp. 60-70, 1978.

[7] T. Marzetta, "Noncooperative cellular wireless with unlimited numbers of base station antennas," IEEE Transactions on Wireless Communications, vol. 9(11), pp. 3590-3600, 2010.

[8] A. Damnjanovic, J. Montojo, Y. Wei, T. Ji, T. Luo, M. Vajapeyam, T. Yoo, O. Song, and D. Mallad, "A survey on 3gpp heterogeneous networks," IEEE Wireless Communications, vol. 18(3), pp. 10-21, 2011.

[9] S. Jafar, "Interference alignment: A new look at signal dimensions in a communication network," Foundations and Trends in Communications and Information Theory, vol. 7(1), pp. 1-134, 2011. 
[10] C. 2012, Cisco Visual Networking Index: Global Mobile Data Traffic Forecast Update, 2011-2016. Signals and Communication Technology, CISCO White Paper, 2012.

[11] V. Cadambe and S. Jafar, "Interference alignment and degrees of freedom of the K-User interference channel," IEEE Transactions on Information Theory, vol. 54(8), pp. 3425-3441, 2008.

[12] M. Maddah-Ali, A. Motahari, and A. Khandani, "Communication over MIMO X channels: Interference alignment, decomposition, and performance analysis," IEEE Transactions on Information Theory, vol. 54(8), pp. 3457-3470, 2008.

[13] V. Cadambe and S. Jafar, "Can 100 speakers talk for 30-minutes each in one room within one hour and with zero interference to each other's audiance?," in Proceedings of Annual Allerton Conference on Communications, Control and Computing, pp. 1141-1148, 2007.

[14] S. Jafar and S. Shamai, "Degrees of freedom region for the MIMO X channel," IEEE Transactions on Information Theory., vol. 54(1), pp. 151$170,2008$.

[15] O. El Ayach, S. Peters, and R. Heath, "The Practical Challenges of Interference Alignment," IEEE Wireless Communications, vol. 13(12), pp. 3542, Feb 2013.

[16] G. R. Group, Universal Mobile Telecommunications System (UMTS); Spacial channel model for Multiple Input Multiple Output (MIMO) simulations. 3GPP TR 25.996 version 6.1.0 Release 6), ETSI TR 125 996 V6.1.0 (2003-09), 2003.

[17] D. Tse and P. Viswanath, Fundamentals of Wireless Communication. Cambridge University Press, 2005.

[18] A. Goldsmith, Wireless Communications. Cambridge University Press, 2005 .

[19] V. Erceg, L. Greenstein, S. Tjandra, S. Parkoff, A. Gupta, B. Kulic, and A. Julius, "An empirically based path loass model for wireless channels in suburban areas," IEEE Journal on Selected Areas Communications, vol. 17(12), pp. 1205-1211, 1999.

[20] H. Holma and A. Toskala, WCDMA for UMTS: HSPA Evolution andLTE. John Wiley and Sons, 2010. 
[21] H. Holma and A. Toskala, LTE for UMTS: OFDMA and SC-FDMA based radio access. John Wiley and Sons, 2009.

[22] B. Clerckx and C. Oestges, MIMO Wireless Networks: Channels, Techniques and Standards for Multi-Antenna, Multi-User and Multi-Cell Systems. Academic Press, 2013.

[23] J. H. Winters, "On the capacity of radio communication systems with diversity in a Rayleigh fading environment," IEEE Journal on Selected Areas in Commununications, vol. 5(5), pp. 871 - 878, 1987.

[24] E. Teletar, "Capacity of multi-antenna Gaussian channels," ATEST Bell Labs, Technical Report, 1995.

[25] G. J. Foschini and M. J. Gans, "On limits of wireless communications in a fading environment when using multiple antennas," Wireless Personal Communications, pp. 311 - 335, 1998.

[26] T. Lo, "Maximum ratio transmission," in Proceedings of IEEE International Conference on Communications, pp. 1310 - 1314, 1999.

[27] A. Grant, "Performance analysis of transmit beamforming," IEEE Transactions on Communications, vol. 53(3), pp. 738 - 744, 2005.

[28] A. Narula, M. Lopez, M. Trott, and G. W. Wornell, "Efficient use of side information in multiple-antenna data transmission over fading channels," IEEE Journal on Selected Areas in Communications, vol. 16(8), pp. 1423 - 1436, 1998.

[29] G. Caire and S. Shamai, "On the achievable throughput of a multiantenna Gaussian broadcast channel," IEEE Transactions on Information Theory, vol. 49(7), pp. 1691 - 1706, 2003.

[30] H. Viswanathan, S. Venkatesan, and H. Huang, "Downlink capacity evaluation of cellular networks with known-interference cancellation," IEEE Journal on Selected Areas in Communications, vol. 21(5), pp. 802 - 811, 2003.

[31] C. Au-Yeung and D. Love, "On the performance of random vector quantization limited feedback beamforming in a MISO system," IEEE Transactions on Wireless Communications, vol. 6(2), pp. 458 - 462, 2007.

[32] G. R. Group, Long Term Evolution, Evolved Universal Terrestrial Radio Access (E-UTRA) Physical Layer, General Descriptions. 3GPP TS 36.201), ETSI TS 36.201 (2011-01), 2003. 
[33] D. Gesbert, M. Kountouris, R. Heath, C.-B. Chae, and T. Salzer, "Shifting the MIMO paradigm," IEEE Signal Processing Magazine, vol. 24(5), pp. $36-46,2007$.

[34] N. Jindal, "MIMO broadcast channels with finite-rate feedback," IEEE Transactions on Information Theory, vol. 52(11), pp. 5045 - 5060, 2006.

[35] T. Yoo and A. Goldsmith, "On the optimality of multiantenna broadcast scheduling using zero-forcing beamforming," IEEE Journal on Selected Areas in Communications, vol. 24(3), pp. 528 - 541, 2006.

[36] S. Shamai and B. M. Zaidel, "Enhancing the cellular downlink capacity via co-processing at the transmitting end," in Proceedings of IEEE Vehicular Technology Conference, vol. 5(5), pp. 1745 - 1749, 2001.

[37] H. Zhang and H. Dai, "Cochannel interference mitigation and cooperative processing in downlink multicell multiuser MIMO networks," EURASIP Journal on Wireless Communications and Networking, vol. 2(1), pp. 222 - 235, 2004.

[38] D. Gesbert, S. Hanly, H. Huang, S. Shamai Shitz, O. Simeone, and W. Yu, "Multi-cell MIMO cooperative networks: A new look at interference," IEEE Journal on Selected Areas in Communications, vol. 28(9), pp. 1380 $-1408,2010$.

[39] A. Carleial, "A case where interference does not reduce capacity," IEEE Transactions on Information Theory, vol. 21(5), pp. 60-70, 1975.

[40] G. R. Group, Universal Mobile Telecommunications System (UMTS); Spacial channel model for Multiple Input Multiple Output (MIMO) simulations Release10. 3GPP TR 25.996 version 6.1.0 Release 10), ETSI TR 125996 V6.1.0 (2011-03), 2011.

[41] T. Han and K. Kobayashi, "A new achievable rate region for the interference channel," IEEE Transactions on Information Theory, vol. 27(1), pp. 49-60, 1981.

[42] H. Sato, "The capacity of the gaussian interference channel under strong interference," IEEE Transactions on Information Theory, vol. 27(6), pp. 786-788, 1981.

[43] R. Etkin, D. Tse, and H. Wang, "Gaussian interference channel capacity to within one bit," IEEE Transactions on Information Theory, vol. 54(12), pp. 5534-5562, 2008. 
[44] A. Motahari and A. Khandani, "Capacity bounds for the Gaussian interference channel," IEEE Transactions on Information Theory, vol. 55(2), pp. $620-643,2009$.

[45] X. Shang, G. Kramer, and B. Chen, "A new outer boundand the noisyinterference-sum-rate capacity for Gaussian interference channels," IEEE Transactions on Information Theory, vol. 5(5), pp. 689-699, 2009.

[46] V. Annapureddy and V. Veeravalli, "Gaussian interference network: Sum capacity in the low interference regime and new outer bounds on the capacity region," IEEE Transactions on Information Theory, vol. 55(7), pp. $3032-3050,2009$.

[47] F. Lombardo, A. Vanelli-Coralli, E. Candreva, and G. Corazza, "Multi gateway interference cancellation techniques for the return link of multibeam broadband satellite systems," in Proceedings of IEEE Global Communications Conference (GLOBECOM), pp. 3425-3430, 2012.

[48] D. Zheng and P. Karabinis, "Adaptive beam-forming with interference suppression and multi-user detection in satellite systems with terrestrial reuse of frequencies," in Proceedings of IEEE Vehicular Technology Conference - Fall, pp. 2637-2641, 2005.

[49] S. Nordholm, J. Nordberg, L. Claesson, and S. Nordebo, "Beamforming and interference cancellation for capacity gain in mobile networks," Annals of Operational Research, vol. 98, pp. 235-253, 2001.

[50] S. Bittner, E. Zimmermann, and G. Fettweis, "Low complexity softinterference cancellation for MIMO systems," in Proceedings of IEEE 63rd Vehicular Technology Conference, pp. 1993-1997, 2006.

[51] M. Breinholt, M. Zoltowski, and T. Thomas, "Space-time equalization and interference cancellation for MIMO OFDM," in Conference Record of the Thirty-Sixth Asilomar Conference on Signals, Systems and Computers, pp. 1688-1693, 2002.

[52] X. Wang and W. Poor, "Iterative (turbo) soft interference cancellation and decoding for coded cdma," IEEE Transactions on Communications, vol. 47(7), pp. 1046-1062, 1999.

[53] Y. Birk and T. Kol, "Coding on demand by an informed source (ISCOD) for efficient broadcast of different supplemental data to caching clients," IEEE Transactions on Information Theory, vol. 52(6), pp. 2825-2830, 2006. 
[54] Y. Birk and T. Kol, "Informed-source coding-on-demand (ISCOD) over broadcast channels," in Proceedings of the Seventeeth Annual Joint Conference of the IEEE Computer and Communications Societies, pp. 12571264, 1998.

[55] M. Anthony and M. Harvey, Linear Algebra: Concepts and Methods. Cambridge University Press, 2012.

[56] S. Jafar and M. Fakhereddin, "Degrees of freedom for the MIMO interference channel," IEEE Transactions on Information Theory, vol. 53(7), pp. 1478 - 1491, 2007.

[57] C. Shi, R. Berry, and M. Honig, "Adaptive beamforming in interference networks via bi-directional training," ArXiv preprint arXiv:1003.4764, p. http://arxiv.org/abs/1003.4764, 2010.

[58] H. Bolcskei and I. Thukral, "Interference alignment with limited feedback," in Proceedings of IEEE International Symposium on Inofrmation Theory, pp. 1759-1763, 2009.

[59] H. A. Suraweera, P. J. Smith, and M. Shafi, "Capacity limits and performance analysis of cognitive radiowith imperfect channel knowledge," IEEE Transactions on Vehicular Technology, vol. 59(4), pp. 1811-1822, 2010 .

[60] K. Ahn and R. Heath, "Performance analysis of maximum ratio combining with imperfect channel estimationin the presence of cochannel interferences," IEEE Transactions on Wireless Communications, vol. 8(3), pp. 1080-1085, 2009.

[61] T. Marzetta, "BLAST training: Estimating channel characteristics for high capacity space-time wireless," in Proceedings of Annual Allerton Conference on Communications, Control and Computing, pp. 958-966, 2009.

[62] D. J. Love, R. Heath Jr, V. Lau, D. Gesbert, B. D. Rao, and M. Andrews, "An overview of limited feedback in wireless communication systems," IEEE Journal on Selected Areas in Communications, vol. 26(8), pp. 1341-1365, 2008.

[63] J. Zheng, R. E. Duni, and B. D. Rao, "Analysis of multiple-antenna systems with finite-rate feedback using high-resolution quantization theory," IEEE Transactions on Signal Processing, vol. 55(4), pp. 1461-1476, 2007.

[64] Y. Isukapalli, J. Zheng, and B. Rao, "Average SEP loss analysis of tranmsit beamforming for finite rate feedback MISO systems with QAM constel- 
lation," in Proceedings of IEEE International Conference on Acoustics, Speech and Signal Processing, pp. III425 - III428, 2007.

[65] W. Santipach and M. Honig, "Asymptotic performance of MIMO wireless channels with limited feedback," in Proceedings of IEEE Military Communications Conference, pp. 141 - 146, 2003.

[66] "Asymptotic capactiy of beamforming with limited feedback," in Proceedings of IEEE International Symposium on Spread Spectrum Technologies and Applications, p. 290, 2004.

[67] Q. Li, E. Lin, and J. Zhang, "MIMO precoding in 802.16e WIMAX," Journal of Communications and Networks, vol. 9(2), pp. 1046-1062, 2007.

[68] B. Hochwald, T. Marzetta, T. Richardson, W. Sweldens, and R. Urabanke, "Systematic design of unitary space-time constellations," IEEE Transactions on Information Theory, vol. 46, pp. 1962-1973, 2000.

[69] B. Mondal, R. Samanta, and R. W. Heath, "Frame theoretic quantization for limited feedback mimo beamforming systems," in Proceedings of IEEE International Conference Wireless Communications and Mobile Communications, pp. 1065-1070, 2005.

[70] J. Roh and B. Rao, "An efficient feedback method for mimo systems with slowly time-varing channels," in Proceedings of IEEE International Wireless Communications and Networking Conference, pp. 760-764, 2004.

[71] J. Roh and B. Rao, "Channel feedback quantization methods for MISO and MIMO systems"," in Proceedings of IEEE International Symposium on Personal Indoor and Mobile Radio Communications, pp. 805-809, 2004.

[72] G. Golub and C. Van Loan, Matrix Computations, 3rd ed. ), Johns Hopkins University Press, 1996.

[73] R. W. Heath, J. Chiang, B. Mondal, and R. Samanta, 11n partial proposal: Quantized precoding with feedback. ), IEEE, 2004.

[74] K. Ko and J. Lee, "Multiuser MIMO user selection based on chordal distance," IEEE Transactions on Communications, vol. 60(3), pp. 649-654, 2012.

[75] J. Andrews, A. Ghosh, and R. Muhamed, Fundamentals of WIMAX: Understanding Broadband Wireless Networking . Prentice Hall, 2007.

[76] S. Loyka, "Channel capacity of MIMO architecture using the exponential correlation matrix," IEEE Communication Letters, vol. 5(9), pp. 369-371, 2001. 
[77] T. Gou and S. Jafar, "Degrees of Freedom of the $\mathrm{K}$ User $\mathrm{M} \times \mathrm{N}$ MIMO Interference Channel," IEEE Transactions on Information Theory, vol. 56(12), pp. 6040-6057, Dec 2010.

[78] M. Pischella and E. Vivier, "Comparison of Distributed Space and Frequency Interference Alignment ," in Proceedings of IEEE International Symposium on Personal, Indoor and Mobile Communications, pp. 10371044, 2010.

[79] N. Lee, L. J-B., and J. Chun, "Degrees of Freedom of the MIMO Y Channel: Signal Space Alignment for Network Coding," IEEE Transactions on Information Theory, vol. 56(7), pp. 3332-3342, July 2010.

[80] W. Shin and B. Clerckx, "Interference Alignment with Limited Feedback on Two-cell Interfering Two User MIMO-MAC," in Proceedings of IEEE International Symposium on Wireless Communications Systems, pp. 566570, 2012.

[81] K. Au-Yeung and D. J. Love, "On the Performance of Random Vector Quantization Limited Feedback Beamforming in MISO Systems," IEEE Wireless Communications, vol. 6(2), pp. 458-462, Feb 2007.

[82] R. Zhou, T. Lu, H. Gao, and W. Long, "A New Limited Feedback Scheme for Interference Alignment in Two-Cell Interfering MIMO-MAC ," in Proceedings of IEEE International Symposium on Personal, Indoor and Mobile Communications, pp. 1-4, 2012.

[83] G. Sridharan and W. Yu, "Degrees of freedom of MIMO cellular networks: Decomposition and linear beamforming design," ArXiv preprint arXiv:1312.2681, p. http://arxiv.org/abs/1312.2681, 2013.

[84] K. Pham and K. Lee, "Interference alignment for multicell multiuser MIMO uplink channels," ArXiv preprint arXiv:1408.4994, p. http://arxiv.org/abs/1408.4994, 2014.

[85] S. Serbetli and A. Yener, "Transceiver Optimization for Multiuser MIMO Systems," IEEE Transactions on Signal Processing, vol. 51(3), pp. 214226,2004 .

[86] N. Lee and W. Shin, "Adaptive Feedback Scheme on K-Cell MISO Interfering Broadcast Channel with Limited Feedback ," IEEE Transactions on Wireless Communications, vol. 10(2), pp. 401-406, Feb 2011.

[87] W. Shin, N. Lee, J.-B. Lim, S. C., and K. Jang, "Interference Alignment Through User Cooperation for Two-Cell MIMO Interfering Broad- 
cast Channels ," in Proceedings of IEEE Global Communications Conference, pp. 120-125, 2010.

[88] R. Tresch and M. Guillaud, "Cellular Interference Alignment with Imperfect Channel Knowledge ," in Proceedings of IEEE International Conference on Communications, pp. 120-125, 2009.

[89] L. Parleza, Debbah and Chaufray, "Opportunistic Interference Alignment in MIMO Interference Channels ," in Proceedings of IEEE International Symposium on Personal, Indoor and Mobile Communications, pp. 120$125,2008$.

[90] C. Suh and D. Tse, "Interference Alignment for Cellular Networks," in Allerton Conference on Communications, Control and Computing, pp. 1037-1044, 2008.

[91] Q. Spencer, A. Swindlehurst, and M. Haardt, "Zero-forcing methods for downlink spatial multiplexing in multiuser MIMO channels," IEEE Transactions on Signal Processing, vol. 52(2), pp. 461-471, 2004.

[92] M. Sadek, A. Tarighat, and A. Sayed, "A Leakage-Based Precoding Scheme for Downlink Multi-User MIMO Channels," IEEE Transactions on Wireless Communications, vol. 6(5), pp. 1711-1716, 2007.

[93] T. Ai and Z. Wu, "Interference mitigation for downlink base station cooperation with joint distributed space-time coding," in Proceedings of IET Wireless Mobile and Computing Conference, pp. 201-206, 2011.

[94] U. Jang, K. Lee, K. S. Cho, and W. Ryu, "Downlink transmit beamforming for intercell interference mitigation with bs cooperation," in Proceedings of IEEE Global Telecommunications Conference, pp. 1-5, 2010.

[95] G. Caire, H. Ramprashad, C. Papadopolous, and C. Pepin, "'multiuser mimo downlink with limited inter-cell cooperation: Approximate interference alignment in time, frequency and space," in Proceedings of Annual Allerton Conference on Communications, Control and Computing, pp. 730-737, 2008.

[96] G. R. Group, CoMP operation for LTE physical layer aspects, Release 11. 3GPP TR 25.996 version 0.0.1), 2011.

[97] L. G. Guedes and M. Yacoub, "Overlapping cell area in different fading conditions," in Proceedings of IEEE Vehicular Technology Conference, pp. 380-383, 1995. 
[98] V. Cadambe and S. Jafar, "Interference alignment and the degrees of freedom of wireless X networks," IEEE Transactions on Information Theory, vol. 550(9), pp. 3893-3908, 2009.

[99] R. Ustok, P. Dmochowski, P. Smith, and M. Shafi, "Aligned interference neutralisation for $2 \times 2 \times 2$ interference channel with imperfect channel state information," in Proceedings of IEEE International Conference on Communications, pp. 5230-5235, 2013.

[100] W. Shin, R. Heath, and B. Clerckx, "Interference alignment with limited feedback on two-cell interfering two user MIMO-MAC," in Proceedings of IEEE International Symposium on Wireless Communication Systems, pp. 566-570, 2012.

[101] R. Zhou, T. L., H. Gao, and W. Long, "A new limited feedback scheme for interference alignment in two-cell interfering MIMO-MAC," in Proceedings of IEEE International Symposium on Personal Indoor and Mobile Radio Communications, pp. 1633-1638, 2012.

[102] X. Feng and Z. Zhong, "The rank of a random matrix," Applied Mathematics and Computation, vol. 185(1), pp. 689 - 694, 2007.

[103] A. Yeung and D. Love, "On the performance of random vector quantization limited feedback beamforming in a miso system," IEEE Transactions on Wireless Communications, vol. 7(1), pp. 458-462, 2007.

[104] Q. Li, X. Lin, and J. Zhang, "MIMO precoding in 802.16e WIMAX," IEEE Journal of Communications and Networks, vol. 9(2), pp. 141-149, 2007.

[105] H. Tataria, M. Shafi, P. Smith, and P. Dmochowski, "General analysis of multiuser MIMO systems with regularized zero-forcing precoding under spatially correlated Rayleigh fading channels ," in Proceedings of IEEE International Conference on Communications, p. 2582-2588, 2016.

[106] H. Tataria, M. Shafi, P. Smith, and P. Dmochowski, "Coordinated two-tier heterogeneous cellular networks with leakage based beamforming," CoRR, vol. vol.abs/1503.01566, 2015.

[107] M. Hahn and M. Klass, "Matrix normalization of sums of random vectors in the domain of attraction of the multivariate normal," The Annals of Probability, vol. 8(2), pp. 262 - 280, 1980.

[108] B. Recht, W. Xu, and B. Hassibi, "Null space conditions and thresholds for rank minimization," Mathematical Programming, vol. 127(1), pp. 175 $-202,2011$. 
[109] R. Ustok, P. Dmochowski, P. Smith, and M. Shafi, "Cooperative interference cancellation for cellular networks with imperfect ccsi," IET Communications, vol. 10(5), pp. 525 - 533, 2016.

[110] R. F. Ustok, P. Dmochowski, P. Smith, and M. Shafi, "Interference alignment for cellular networks with SLRINR-based precoders," Submitted to IET Communications, pp. -, --

[111] W. Shin, N. Lee, J.-B. Lim, and K. Jang, "On the design of interference alignment scheme for two-cell MIMO interfering broadcast channels," IEEE Transactions on Wireless Communications, vol. 10(2), pp. 437-442, 2011.

[112] W. Shin, N. Lee, J.-B. Lim, and K. Jang, "Interference alignment through user cooperation for two-cell MIMO interfering broadcast channels," in Proceedings of IEEE Proceedings of IEEE Global Communications Conference, pp. 120-125, 2010.

[113] Z. Wang and W. Chen, "Regularized zero-forcing for multiantennabroadcast channels with user selection," IEEE Wireless Communications Letters, vol. 1(2), pp. 129-132, 2012.

[114] F. Yuan and C. Yang, "Equivalence of SLNR precoder and RZF precoder in downlink MU-MIMO systems," ArXiv e-prints, [online] Available: http://arxiv.org/abs/1202.1888, pp. -, 2012.

[115] M. Sadek, A. Tarighat, and A. Sayed, "Active Antenna Selection in Multi User MIMO Communications," IEEE Transactions on Signal Processing, vol. 55(4), pp. 1498-1510, 2007.

[116] S. Hasan, A. Hayat, and H. F., "On the downlink sinr and outage probability of stochastic geometry based lte cellular networks with multi-class services," in Proceedings of IEEE International Conference on Computer and Information Technology, pp. 65-69, 2015.

[117] P. Viswanath and V. Anantharam, "Optimal Sequences and Sum Capacity of synchronous CDMA Systems," IEEE Transactions on Information Theory, vol. 45(6), pp. 1984-1991, 1999.

[118] S. Ulukus and R. D. Yates, "Iterative Construction of Optimum Signature Sequence Sets in Synchronous CDMA Systems," IEEE Transactions on Information Theory, vol. 47(5), pp. 1989-1998, 2001.

[119] G. S. Rajappan and M. L. Honig, "Signature Sequence Adaptation for DS-CDMA with Multipath," IEEE Journal on Selected Areas in Communications, vol. 20(2), pp. 384-395, 2002. 
[120] R. Horn and C. Johnson, Matrix Analysis. Cambridge University Press, 1985.

[121] B.-C. Lim, W. Krzymien, and C. Schlegel, "User selection methods for multi-user downlinks with multiple antenna terminals," in Proceedings of IEEE Singpaore International Conference on Communications Systems, pp. 1-5, 2006.

[122] N. Schrammar and P. Hoeher, "Fair user selection for zero-forcing precoding in multi-user miso systems," in Conference Record of the Forty-Third Asilomar Conference on Signals, Systems and Computers, pp. 1412-1415, 2009 .

[123] Y. Cao and V. Li, "Scheduling Algorithms in Broadband Wireless Networks," Proceedings of IEEE, vol. 89(1), pp. 76-87, 2002.

[124] A. Aitzaz, A. Papathanassiou, E. Serpeddin, P. Smith, and M. Shafi, “ LTE Codebook Capacity Loss for Single-Cell Multi User MIMO Channels ," pp. 1902-1906, 2012.

[125] R. Jains, D. Chui, and W. Hawe, "A quantative measure of fairness and discrimination for resource allocation in shared computer system," $A C M$ Transactions on Computer Systems, pp. 1 - 35, 1984.

[126] U. Salim and D. Slock, "Performance of different user selection algorithms for transmit power minimization," in Conference Record of the Forty-Third Asilomar Conference on Signals, Systems and Computers, pp. 1469-1473, 2009 .

[127] T. Yoo and A. Goldsmith, "On the Optimality of Multiantenna Broadcast Scheduling Using Zero-Forcing Beamforming," IEEE Journal on Selected Areas in Communications, vol. 24(3), pp. 528-541, 2006.

[128] T. Yoo and A. Jindal, N.and Goldsmith, "Multi-antenna downlink channels with limited feedback and user selection," IEEE Journal on Selected Areas in Communications, vol. 25(7), pp. 1478-1491, 2015.

[129] S. Hur, B.-C. Jung, and B.-D. Rao, "Sum rate enhancement by maximizing SGINR in an opportunistic interference alignment," in Conference Record of the Forty-Fifth Asilomar Conference on Signals, Systems and Computers, pp. 354-358, 2011.

[130] C. Zhong, T. Ratnarajah, S. Jin, and K.-K. Wong, "Performance analysis of optimal single stream beamforming," IEEE Journal on Selected Areas Communications, vol. 30(8), pp. 1415-1427, 2012. 
[131] X. Chen and R. Yin, "Performance analysis for physical layer security in multi-antenna downlink networks with limited csi feedback," IEEE Wireless Communications Letter, vol. 2(5), pp. 503-506, 2013.

[132] S. Chao, H. Chae, K. Huang, D. Kim, and V. Lau, "Efficient feedback design for interference alignment in MIMO interference channel," in Proceedings of IEEE Vehicular Technology Conference, pp. 1-5, 2012.

[133] X. Rao, L. Ruan, and V. Lau, "Limited feedback design for interference alignment on MIMO interference networks with heterogenous path loss and spatial correlations," IEEE Transactions on Signal Processing, vol. 61(10), pp. 2598-2607, 2013.

[134] X. Chen and C. Yuen, "On interference alignment with imperfect csi: Characterizations of outage probability, ergodic rate and SER," IEEE Transactions on Vehicular Technology, vol. 65(1), pp. 47-58, 2016.

[135] E. Erten, R. Zandona-Scheneider, and A. Reigber, "Statistical characterisation of the maximum eigenvalue of a wishart distribution with application to multi-channel SAR systems," in Proceedings of International Workshop on Applications of Polarimetry and Polarimetric Interferometry, pp. 1-4, 2009.

[136] I. Johnstone, "On the distribution of the largest eigenvalue in principal component analysis," The Annals of Statistics, pp. 295-327, 2001.

[137] A. Swindlehurst, E. Ayanoglu, P. Heydari, and F. Capolino, "Milimeterwave massive MIMO: The next wireless revolution," IEEE Communications Magazine, vol. 52(9), pp. 56-62, 2014.

[138] Z. Pi and F. Khan, "An introduction to milimeter-wave mobile broadband systems," IEEE Communications Magazine, vol. 49(6), pp. 101-107, 2011.

[139] T. Rappaport, S. Sun, R. Mayzus, H. Zhao, Y. Azar, K. Wang, G. Wong, J. Schulz, M. Samimi, and F. Gutierrez, "Milimeter wave mobile communications for 5G cellular: It will work!," IEEE Access, vol. 1(1), pp. 335-349, 2013.

[140] Y. Niu, Y. Li, D. Jin, L. Su, and A. Vasilakos, "A survey of milimeter wave (mmwave) communications for 5g: Opportunities and challenges," ArXiv preprint arXiv:1502.07228v1, p. http://arxiv.org/abs/1502.07228, 2015 .

[141] H. Tataria, P. Smith, L. Greenstein, P. Dmochowski, and M. Shafi, "Performance and Analysis of Downlink Multiuser MIMO Systems With Reg- 
ularized Zero-Forcing Precoding in Ricean Fading Channels ," in Proceedings of IEEE International Conference on Communications, pp. 11851192, 2016.

[142] N. Lee and R. Heat Jr., "Advanced interference management technique: Potentials and limitations," ArXiv preprint arXiv:1501.00080v2, p. http://arxiv.org/abs/1501.00080, 2015.

[143] T. Thornburg, T. Bai, and R. Heath Jr., "Performance analysis of mmWave AdHoc networks," ArXiv preprint arXiv:1412.0765v2, p. http://arxiv.org/abs/412.0765, 2016.

[144] A. Alkhateeb, G. Leus, and R. W. Heath Jr, "Limited feedback hybrid precoding multi-user milimeter wave systems," ArXiv preprint arXiv:1409.5162v2, p. http://arxiv.org/abs/1409.5162v2, 2015.

[145] L. Zhou and Y. Ohashi, "Low complexity linear receivers for mmwave LOS-MIMO systems with uniform circular arrays," in Proceedings of IEEE Vehicular Technology Conference, pp. 1-5, 2014.

[146] L. Zhou and Y. Ohashi, "Performance analysis of mmwave LOS-MIMO systems with uniform circular arrays," in Proceedings of IEEE Vehicular Technology Conference, pp. 1-5, 2015. 